\title{
A FAIRYTALE ENDING?
}

Violence in Young Adult Dating Relationships in New Zealand:

Testing the utility of Johnson's typology

\author{
by
}

Fleur McLaren

\begin{abstract}
A thesis
Submitted to Victoria University of Wellington

in fulfilment of the requirements for the degree of

Doctor of Philosophy

in Criminology
\end{abstract}

Victoria University of Wellington

02 March 2021 


\section{Abstract}

Dating violence is a serious social problem that causes significant harm and negative outcomes for young adults (Shorey, Cornelius, \& Bell, 2008). Attempts to explain dating violence have often treated those who experience it as a homogeneous group, with theorists divided over the question of gender symmetry versus male dominance as perpetrators. While family conflict researchers advocate gender symmetry, most feminist researchers stress differential offending rates that reflect current gender inequalities.

A more sophisticated conceptual framework is required that captures the heterogeneous and complex nature of violence in dating and other interpersonal relationships (Lewis \& Fremouw, 2001). This has led a number of researchers to develop empirically-based theories and models that attempt to reconcile different findings across studies. One of these is a typology of violence developed by Michael Johnson (2008) as a way to reconcile the differences between studies.

This thesis applies Johnson's typology to the context of young adult dating relationships in New Zealand with the aim of understanding more about the role of gender within intimate partner relationships. I utilised a mixed methods approach that included an extensive literature review, key informant interviews, an online survey, and in-depth qualitative interviews.

This research identified two overarching findings. First, there was overwhelming evidence that a large majority of young adults experience some form of violence in a dating relationship, from more minor experiences to dating relationships characterised by severe and terrifying violence. The data demonstrate how dating violence is linked to the social norms of a youth culture characterised by high alcohol use and expectations around 'hooking-up', with contemporary technology adding to the complexities facing young adults as they navigate this terrain.

The second main finding from this study was that there was positive support for Johnson's (2008) typology in that there are different types of violence in intimate relationships, in this case in dating relationships. The interview data suggest that the complexity of dating violence is better captured through such a typological 
framework that does not view all dating violence as a unitary phenomenon. My application of Johnson's typology provides a narrative that accounts for both dominant traditional gendered violence and also for violent relationships characterised by mutual expressions of conflict and violence. There are, however, some risks associated with the use of such typologies that I also identify and address.

This study holds particular significance as one of the few New Zealand-based analyses of violence occurring in the context of contemporary New Zealand dating relationships. The findings provide strong support for the need to focus on a range of interventions and programmes delivered to young adults who are experiencing different types of violence. They also underscore the importance of critically analysing media messages, challenging traditional gender norms, and confronting attitudes and beliefs that support and validate the use of violence in dating relationships. 


\section{ACKNOWLEDGEMENTS}

This thesis has been a long time in coming. It has spanned a number of years and the birth of my two children. Throughout the process I have had many people support me so there are a number of people for whom I am eternally grateful for their wisdom and support.

First, and most importantly are the young adults who took part in this study. Without their time and honesty there would be no data to inform this work. They shared openly with me about their experiences, some of them very personal stories that demonstrated courage and resilience. And the key informants, a wonderful group of professionals and academics who were busy but took the time to hear my ideas, provide wisdom and offer suggestions that informed the direction of my work.

Throughout this whole process I have had the pleasure of being guided and supported by two amazing supervisors Professor Jan Jordan and Dr Nokuthaba Sibanda. Your patience, encouragement and enduring positivity assisted me through to the end, to achieving this goal. My sincerest thanks to you both.

Over the years that I have worked on this I have had a number of amazing colleagues who have supported me and helped me to persevere. There is not enough space to name them all but I am extremely grateful for their support and understanding. I am also extremely grateful to Deborah O'Kane for proofing the $\mathrm{PhD}$ in its entirety. To my family and friends. My two beautiful girls Taylor and Riley, I spent many hours at the computer when I should've been spending time with you, but I hope you are both proud of your mamma and can see that you too can achieve whatever you put your mind to. To Jason, who was by my side encouraging me, keeping me focused, and who gave me the time and space to do this.

I also want to thank my Mum and Dad who taught me the values of patience, perseverance and hard work; you have both made me who I am today. To my brothers and my nana Eileen Fleury, who always supported me from the sidelines. Finally, my amazing friends who have been there when I needed to laugh or cry, when I needed encouragement, or a distraction and to provide me with strength when I needed it. In memory of Sam Janes (Granddad), Marie Janes (Grandma) and Colin Fleury (Grandpa). 


\section{CONTENTS}

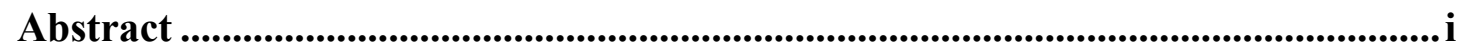

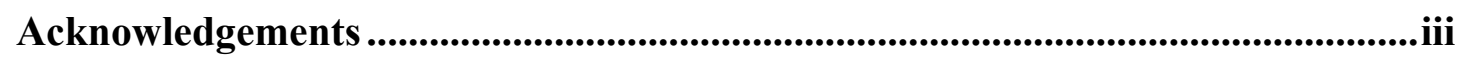

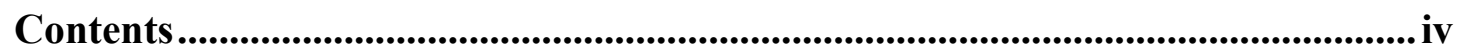

List of tables and figures .....................................................................................................viii

Chapter One - Setting the context for this thesis ................................................1

My interest in dating violence, why focus on this? .................................................................. 3

Young adulthood, a time and place........................................................................................5

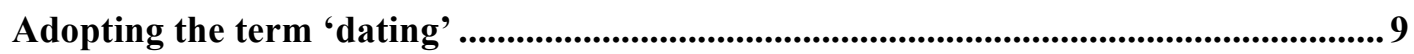

Defining dating violence ............................................................................................................... 12

Dating violence - its inherent links to intimate partner violence ..................................... 14

Research aims and research questions .................................................................................... 19

Thesis format..................................................................................................................................... 19

Chapter Two - Dating violence, a little-known social problem with big implications ......................................................................................................................... 21

Prevalence of violence in dating relationships ........................................................................22

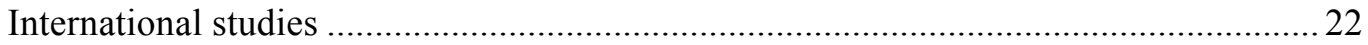

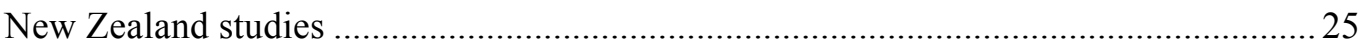

Gender symmetry - the great controversy ...................................................................27

Explaining the differences in the role of gender - a focus on methods, samples and

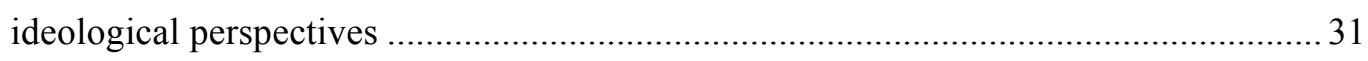

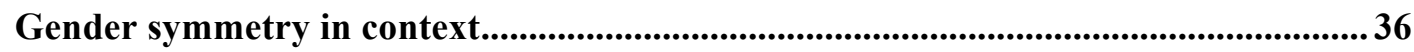

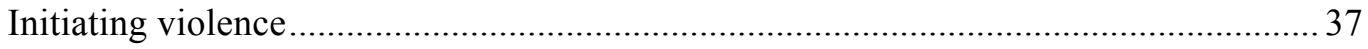

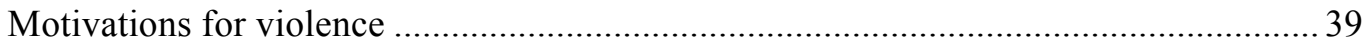

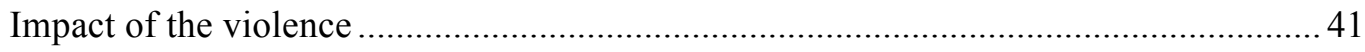

Reconciling the differences - an inclusive description of intimate partner violence... 43

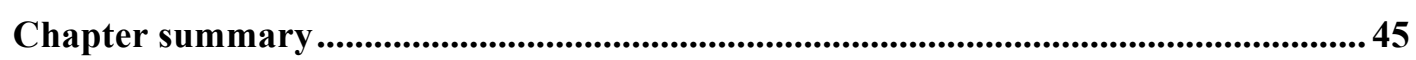

Chapter Three - Reconciling the difference, a typological approach ................... 47

Johnson's development of his typology.............................................................................49

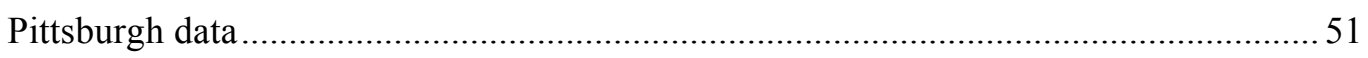

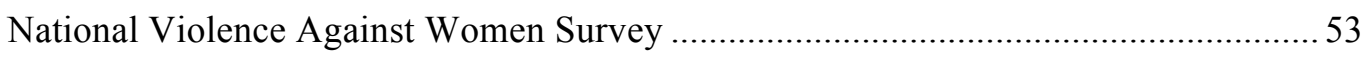

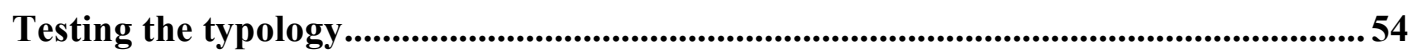

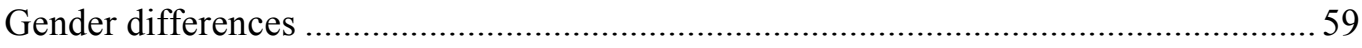


Chapter Four - Method ...........................................................................................65

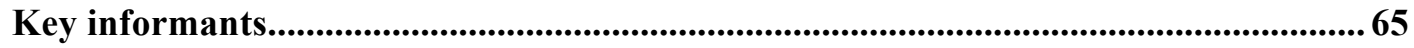

Research approach ...................................................................................................................6 67

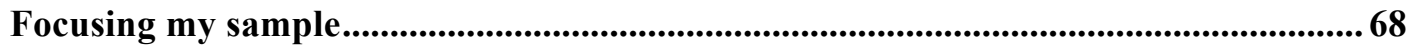

Quantitative online survey ................................................................................................69

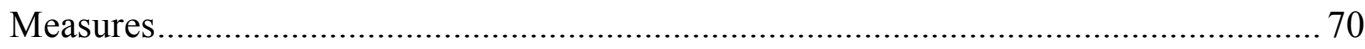

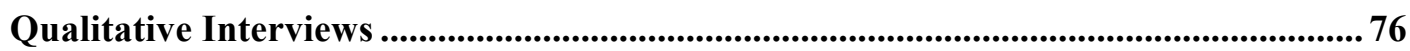

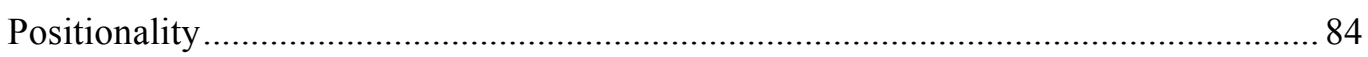

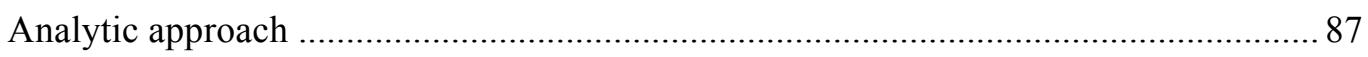

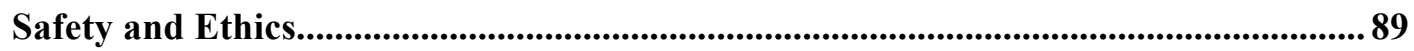

Chapter summary ......................................................................................................................99

Chapter Five - Experience of dating violence, is it really that common? ............ 92

Section One: Demographics................................................................................................92

Section Two: Experience of violence - as victims and as perpetrators .........................99

Frequency of violence - overall count ........................................................................ 98

Distribution of response to each CTS2 item ................................................................. 99

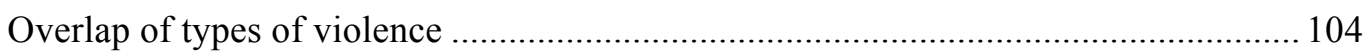

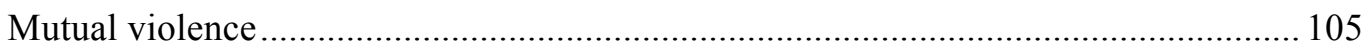

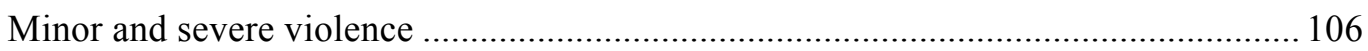

Section Three: injury from dating violence...........................................................110

Experiencing injury as a result of dating violence .................................................... 110

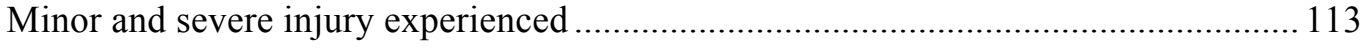

Chapter summary ……….................................................................................................................. 115

Chapter Six - Replicating Johnson's typology ........................................................... 117

Section One: Levels of coercive control ............................................................................... 117

Classification of high and low coercive control....................................................... 120

Section Two: Creation of the typology.............................................................................. 122

Section Three: Demographic and relationship characteristics ..................................... 124

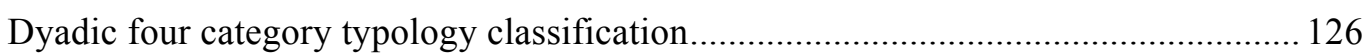

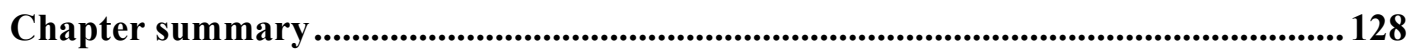

Chapter Seven - Current social and cultural context of dating............................ 129

Section One: The current dating context in New Zealand............................................. 130

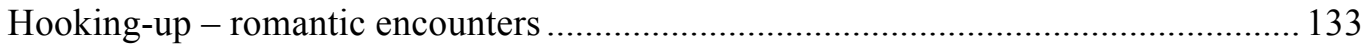

Reasons and motivations for being in a relationship.................................................. 138 
Living together and the roles in the household

Section Two: Impact of technology, social media and popular culture....................... 147

The role of technology, the internet and the media ............................................. 148

Media supporting and glamorising control and violence .................................. 154

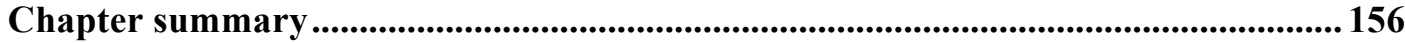

Chapter Eight - Conflict and violence in dating relationships ............................ 158

Section One: Participants' experience non-physical dating violence........................ 159

Monitoring and control through technology and social media................................ 159

Men maintaining an image of being a bloke ........................................................ 160

Men change their behaviour in public versus in private ......................................... 162

Use of threats and manipulative language....................................................... 166

Section Two: Participants' experiences of physical and sexual violence.................... 171

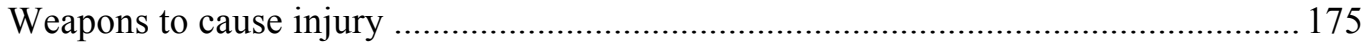

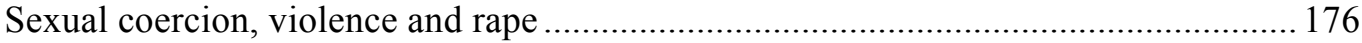

Chapter summary ............................................................................................................ 183

Chapter Nine - Attitudes and beliefs and the context of dating violence........... 185

Section One: Minimising and justifying dating violence......................................... 185

Victims' justification of their partner's use of violence.......................................... 186

Perpetrators' justification of their use of violence ................................................ 189

Section Two: A story of context............................................................................... 195

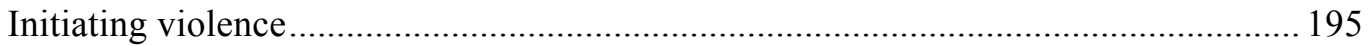

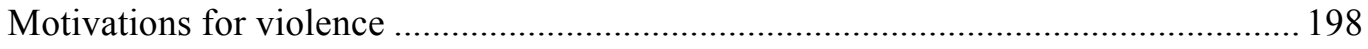

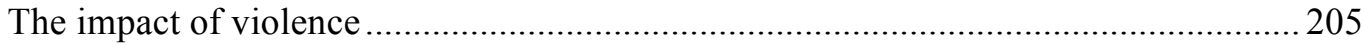

Chapter summary ........................................................................................... 211

Chapter Ten - Synthesis of findings ...........................................................2212

The social context $-\mathbf{a}$ backdrop to dating and violence.............................................. 212

The high rate of dating violence in New Zealand ........................................................ 214

The utility of Johnson's typology ....................................................................216

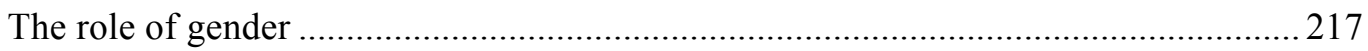

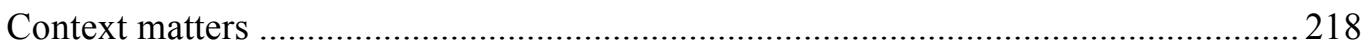

The role of technology and social media .................................................................. 224

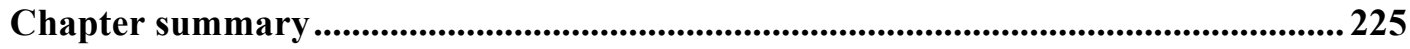

Chapter Eleven - Final thoughts, next steps ......................................................227

What did this study confirm? ............................................................................ 229

Different types and experiences of dating violence .........................................229 
Influence of technology and media

Relaxed versus traditional attitudes, beliefs and behaviours......................................223

Limitations of this study.............................................................................................................. 233

Strengths of the current research........................................................................................... 237

Future research areas and possibilities......................................................................... 238

Practice Implications .............................................................................................................. 241

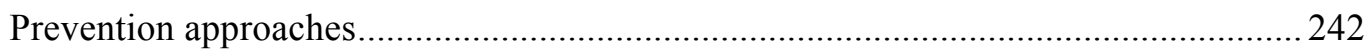

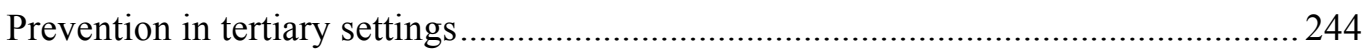

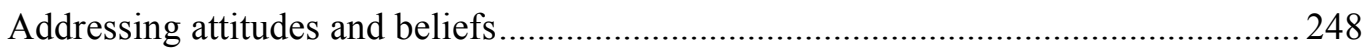

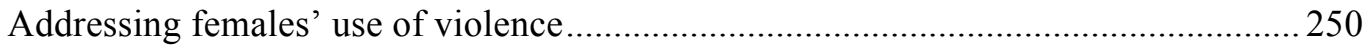

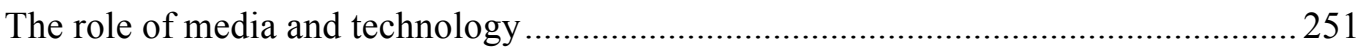

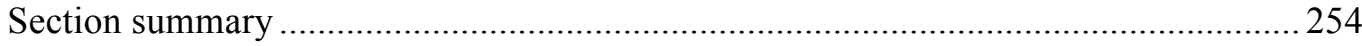

Conclusion and final reflections ..........................................................................................255

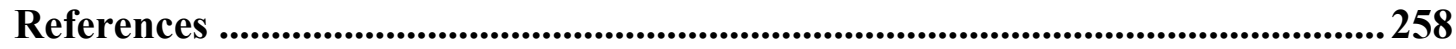

Appendices ....................................................................................................................2279

Appendix 1: Key informants and their roles and expertise ............................................. 279

Appendix 2: Ethics approval ................................................................................................... 281

Appendix 3: Survey Information sheet.......................................................................... 282

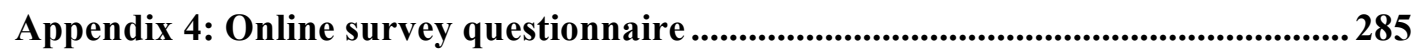

Appendix 5: In-depth interview information sheet ....................................................... 295

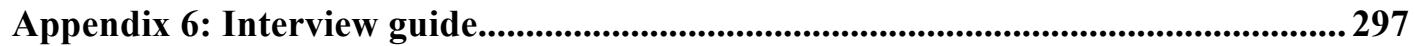

Appendix 7: Copy of the consent form ...............................................................................299

Appendix 8: Description of the interview participants and their partners typology classification ......................................................................................................................................300

Appendix 9: Website prototype detailing the research and seeking research participants for the online survey ….........................................................................................302 


\section{LIST OF TABLES AND FIGURES}

Table 1 Description of the 16 interview participants 79

Table 2 Demographic characteristics for respondents and their partners 94

Table 3 Characteristics of the relationship 97

Table 4 The number of respondents who have experienced violence between an intimate partner over the previous 12 months 98

Table 5 The distribution of responses to each of the CTS2 items over the previous 12

$\begin{array}{ll}\text { months } & 99\end{array}$

Table 6 The number of respondents who have experienced violence between an intimate partner over the previous 12 months by type of violence

Table 7 Respondents' experience of minor and severe violence between an intimate partner over the previous 12 month

Table 8 The number of respondents who experienced injury by an intimate partner over the previous 12 months

Table 9 The distribution of response to each injury item from the CTS2 in the previous 12 months

Table 10 The number of items Respondents experienced as a victim and perpetrator of Injury in the previous 12 months

Table 11 Respondents' experience as a victim and perpetrator of minor and severe injury over a 12-month period

Table 12 The distribution of responses to each of the Coercive Control Scale items over the previous 12 months

Table 13 The number of items of coercive control used by respondents and their partner in the previous 12 months

Table 14 The distribution of participants and their partner into level of control used in the previous 12 months

Table 15 Characteristics of the respondents and relationship descriptions by typology classification 
Figure 1 Experience of one or more types of violence for victims and perpetrators of dating violence in the previous 12 months

Figure 2 The reciprocity of violence for victims and perpetrators of dating violence in the previous 12 months

Figure 3 Crossover of minor and severe violence experienced between and intimate partner over the previous 12 months

Figure 4 Crossover of minor and severe injury experienced by an intimate partner over the previous 12 months

Figure 5 Classification of participants and their partners into Johnson's typology 123 Figure 6 The dyadic interaction of the individual categories to create the four category typology 


\section{CHAPTER ONE - SETTING THE CONTEXT FOR THIS THESIS}

It was midday on the $9^{\text {th }}$ of January 2008. There was a knock at the door. I looked out the window and couldn't see a car in the drive so peered around to see Clayton Weatherston standing there. I opened the door and he came in. I didn't hear what he said but recall Sophie saying she was really busy and running late. If he wanted to talk it would have to be in her room while she continued packing.

Sophie's bedroom is directly above the kitchen and normal conversation can be heard as faint murmuring with any raised voices heard easily. I felt apprehensive and instinctively turned off the radio. I didn't want to interfere as I knew Sophie would be angry with me. I listened but I couldn't hear anything, not even a murmur. It wouldn't have been more than five minutes when I heard the bathroom door close and Sophie appeared in the kitchen. I said, "What's going on?" Sophie said, "I don't know what he wants. He's just sitting there not saying a word." I told her to get rid of him as she was running late and had heaps to do before going out. I suggested that maybe he just wanted to make amends over the assaults and ensure she wasn't going to report him to the police. The toilet flushed and Sophie said I was probably right and she would get him to go.

She went up the stairs and I heard the door close. This was followed by a terrible scream and Sophie shouting, "Don't Clayton, don't Clayton." I tore up the stairs and heard Sophie screaming and screaming. It was the most ungodly noise. I kicked and belted at the door and told him to open it but he didn't. I had to get in so raced back to the kitchen to get a meat skewer and my cellphone. And as I raced back up the stairs I dialled 111, but have no recollection of doing so.

The door handle had a small hole in it as a safety measure to release the locking mechanism. While I was trying to steady my hands to get the skewer into the tiny hole, I could hear a rhythmic thumping. I immediately thought 
Clayton was raping Sophie on the bed and it was the headboard banging against the wall. She was still screaming and screaming. It just went on and on.

I heard Sophie make two gasping sounds - then no more noise other than this horrible thumping.

On getting the door open I saw poor Sophie lying on the floor and I knew instantly she was dead.

Weatherston was kneeling, sort of straddled over her, stabbing her violently in the chest. Not pausing, he continued stabbing Sophie with his right hand while pushing the door closed with his left. He never said a word. The right side of Sophie's face and neck had been viciously stabbed and she looked strange. Although the room was already covered in blood, Sophie was a stark white colour. You don't go white that quickly when you die but the savagery of his attack was such that Sophie had lost a massive amount of blood very rapidly. Her major arteries and veins had been severed and the forensic pathologist described the blood loss as "torrential bleeding that was inevitably lethal". (NZ Herald, 2011)

This is an account of the tragic murder of Sophie Elliott by her ex-boyfriend as described by her mother, Lesley Elliott. The event took place in Dunedin, New Zealand. Her ex-boyfriend was 33-year-old Clayton Weatherston who, on the day of the $9^{\text {th }}$ of January 2008, took 22-year-old Sophie's life by inflicting 216 stabbing and cutting wounds on her. The relationship between Sophie and her ex-boyfriend had lasted just a few months, between June 2007 and December 2007 after regular "ups and downs" and periods of "all on or all off" (Booker, 2009).

Sophie's story demonstrates the devastating impact of dating violence and the potential for it to result in significant harm and even end in homicide. While this is an example of dating violence at the severe end of the continuum, Sophie's story also illustrates behaviours that underpin the wider experience of those in violent dating relationships. 
Dating violence is a serious social problem with physical violence affecting as many as $40 \%$ of young adults in New Zealand every year (Cale, Tzoumakis, Leclerc, \& Breckenridge, 2016; Straus, 2004; Moffitt, Robins, \& Caspi, 2001). While dating can be a positive experience, a growing body of studies demonstrate that violence in dating relationships is a common experience that causes significant harm and negative outcomes for young adults (for example, Marganski \& Melander, 2018; Shorey, Meltzer, and Cornelius, 2010; Shorey, Cornelius, \& Bell, 2008; Harned, 2002; Jackson, 1998a). The body of research that demonstrates this high prevalence and the serious impacts of dating violence was one of the motivations for this thesis.

\section{MY INTEREST IN DATING VIOLENCE, WHY FOCUS ON THIS?}

I first thought about the research topic while out running one morning around Oriental Bay in Wellington. Running can be dangerous! While I was enjoying the early morning air I was reflecting on some recent family violence studies I was involved in at work and my own personal observations of close friends, whose siblings appeared to be following different paths as they engaged in relationships and navigated from adolescence into the more independent young adult phase of their lives.

My observations were of families where parents modelled respect and love and did not condone violent behaviour but where their children, as young adults, ended up in a violent relationship. It appeared that there were very different relationship attitudes and behaviours held across young adult siblings, even when they had been raised in the same households. It was not surprising that different children had different strengths and temperaments, but to have such seemingly fundamentally different attitudes and behaviours, in the context of dating relationships, was fascinating and concerning.

I began to look more intensely at what was influencing how young adults approached relationships and focused on observing patterns within healthy versus more volatile and violent relationships. Eventually I developed my research question which centred on understanding the types, motivations and outcomes of dating violence and the meaning that young adults in New Zealand attribute to this dating violence. My focus on young adults was in part due to the observations of my friends' siblings, but also through the start of my literature search where it became quickly apparent that there 
was little research that specifically focused on this age group in New Zealand. We knew so little about young adults and their experience in dating relationships.

I began to expand the focus of my thesis as my literature review progressed; there were many questions and places to take this topic. For one, I was not convinced that dating violence was primarily perpetrated by men; I felt a need to challenge the notion that men are violent, and that women are only violent in the context of self-defence. From my observations and work in this area this did not seem to capture the experiences of a number of young adult relationships I was aware of. Unlike the violence seen in agency samples, which was more often committed by men in the context of strong patriarchal and gendered beliefs, what I saw in discussions with young adults in dating relationships was that women also could be manipulative and volatile, and that both men and women-initiated conflict and fights.

Through my review of the literature, key informant conversations and discussions with young adults, I expanded the complexity with which I began to view dating violence. In fact, for me, this thesis represents a journey where my viewpoints have changed. Of significance is the development of my view on the gendered nature of dating violence. When I first broached this thesis, it was from a more gender-neutral lens. As I discuss further in Chapter Four on positionality, I was raised in a family where there was no violence and there was equality in how my parents ran the household together and in their roles as working parents. As a result, when I began this thesis, I approached this study with a more neutral position on the role of gender in violence. Through my key informant discussions and engagement with relevant literature I started to shift and expand my perspective. When I was collecting the interview data, the true extent of gendered beliefs and norms became harder to ignore, in tandem with the persuasive coercive controlling behaviours and violence experienced by some young women. This led me more recently to apply a gendered perspective. As a result, the lens represented in this thesis is one that has emerged from a starting place that lacked a gendered framework and I know that if I was to begin this journey today, my starting point would be more clearly reflect the gendered perspective that I ended my journey with. However, what I have captured is a balance that I believe represents the range of experiences of dating violence shared by the young adults who participated in this study, and reflective of where I have come to over my time completing this work. 
It was through my review of the literature and investigating the evidence on the role of gender in the experiences of IPV that I came across Johnson's typology of domestic violence, which is covered in detail in Chapter Three. At this point it was 2010, just two years after he published A Typology of Domestic Violence: Intimate Terrorism, Violent Resistance, and Situational Couple Violence (Johnson, 2008). For me, Johnson's desire to reconcile the differences between studies that found gender symmetry and those that did not made a lot of sense and I felt it was a critical theoretical link in moving towards a fuller understanding of the range of types and experiences of violence in dating, and other, intimate relationships.

The primary aim of the quantitative data analysis was to measure the level of violence experienced in dating relationships by a New Zealand population sample and to interrogate the validity of Johnson's (2008) typology. I replicated Johnson's (2008) typological framework and his corresponding analysis protocol to examine the distribution of the types of violence occurring in dating relationships (see Chapter Six).

This led to my specific focus in this thesis to establish what 'dating' means for young New Zealanders, how this was constructed within the current New Zealand context and to assess the utility of Johnson's typology in understanding the complexity of dating violence experiences in New Zealand.

\section{YOUNG ADULTHOOD, A TIME AND PLACE}

Young adulthood was the stage I wanted to focus on. This is when young people are seeking status as an adult and are engaged in the 'adult world' - leaving home, finishing school and entering tertiary education and the workforce (Schulenberg \& Schoon, 2012). It is also when young people often establish more long-term relationships and/or relationships where they take on roles and responsibilities close to that of married couples. This includes living together, jointly owning possessions and taking on particular roles in a relationship (Bonnie, Stroud, \& Breiner, 2015).

Young adulthood is a time that typically aligns with the stage between the late teenage years and mid-to-late twenties. It has also been referred to as emerging adulthood as young people navigate their way from adolescence into adulthood, although the specific criteria for successful transition to adulthood are not entirely well defined (Petter, 2015). 
The concept of young adulthood is a recent phenomenon as the attainment of rigid social markers of adulthood, such as moving out of home, gaining employment, establishing romantic relationships and making decisions about having children, began to decline or were delayed to older ages (Schulenberg \& Schoon, 2012). Young people can now take multiple pathways to attain these social markers or milestones that historically determined adulthood, thus blurring when adulthood is reached and providing young adults with a range of choices and an opportunity for self-direction and agency (Stone, Berrington, \& Falkingham, 2014).

An example of the change in attaining social markers that signal the achievement of adulthood is the decline in the numbers getting married and the increase in age of those who do enter into marriage. For example, in New Zealand the rate per 1,000 people aged 16 years and over who were married dropped from 27.7 in 1971 to 20.2 in 2016, with the median age of marriage for women increasing from 21.2 years in 1971 to 30.5 years in 2016 and for men from 23.5 years to 32.4 years (Statistics New Zealand, 2017). Likewise, data from the New Zealand Census shows that the number of children who are living in two parent households where their parents are not married has risen from nine percent in 1991 to 21 percent in 2013 (Mitchell, 2016).

The changing patterns of progression through young adulthood and the acceptance of a wide range of pathways can foster resilience and the ability to build skills and learn from experiences that will continue to support young adults through their adult years (Schwartz, 2016). While there are benefits to having more decisions and pathways into 'successful adulthood', the drawback is that this can create a context for more risk taking and risky behaviours. This can be seen with high rates of alcohol and drug abuse, sexual risk taking and increased rates of violence experienced by young adults (Schwartz, 2016). In New Zealand, those aged 18-25 have the highest rates of intentional self-harm and suicide compared to any other age group (Ministry of Health, 2012, in Petter, 2015).

The consumption of alcohol is also linked to increased risk taking and greater negative health outcomes for young adults (O'Keefe, Bhatti, Bajwa, DiNicolantonio, \& Lavie, 2014). The drinking culture in New Zealand is supported by a strong acceptance and normalisation of drinking to intoxication and it has also been supported by a proliferation of advertising, the development of new alcoholic beverages that appeal to 
the younger drinkers (e.g., pre-mixed ready-to-drink products or RTDs), a relaxation of alcohol laws, lowering the age of legal purchase of alcohol, longer trading hours for the sale of alcohol and an expansion of public places for young people to seek both leisure and pleasure (James, 2010). These factors have supported the increase in the number of young adults planning to go out and get excessively drunk or 'wasted'.

Within this strong drinking culture there appears to be an implicit link to participation in the night-time economy (going out and getting drunk), including engagement in casual sex and hook-ups (Hutton, Wright, \& Saunders, 2013). Danube, Vescio and Davis (2014) describe the university years as a time where young adults find there is minimal supervision and they report that as many as 30 percent of college undergraduates in the United States of America (USA) engage in intercourse with someone they do not know or have only briefly known. While this sort of casual sexual encounter does not necessarily result in negative impacts on young adults, it can create a context for unsafe sexual practices including not using condoms or experiencing coercive sexual experiences, particularly when excess alcohol has been consumed.

Alongside the diversity of choices for young adults, and the increased risk associated with engaging in the night-time economy, there are other influencing factors on young adults' choices and behaviours. While peers and romantic partners play an important role in influencing behaviours and setting norms, over the past two decades young people's interactions, self-identities and relationships have also been significantly influenced and shaped by the media including television, the Internet, magazines, reality television and by reality stars (Hendrickson, 2007).

Social media allows young adults to constantly assess what their peers are engaging in, to gauge social norms and to measure their standings in relation to peers. Manago, Ward, Lemm, Reed and Seabrook (2015) discuss how social networking sites in particular cultivate behaviours that see young adults curating flattering images of themselves and posting images that are sexually provocative to gain attention from both the same and opposite sex. Constant messaging and advertising around what women and men should look like, how they should behave and what consumer items they should acquire to be seen as successful is embedded in social media and social networking sites. Such forms of media also glamorise and normalise violence and sexualised behaviours, making it harder for young adults to navigate their way to 
healthy images of themselves, their peers and positive relationships (Office of Film and Literature Classification, 2017).

This is amplified by the increasing accessibility to pornography and other sexually explicit materials (SEM) through the growth and reach of the internet (Lambert, Negash, Stillman, Olmstead, \& Fincham, 2012; Classification Office, 2020). The effects of viewing sexually explicit material are not entirely negative, such as studies that show the positive influence of sexually explicit material on attitudes to sexuality (McKee, 2007). However, there is a substantial body of research that provides evidence of a number of negative issues associated with viewing sexually explicit material. These include SEM's impact on relationship satisfaction, attitudes towards women and violence and a greater acceptance of rape (Braithwaite, Coulson, Keddington, \& Fincham, 2015).

On the one hand, contemporary young people are more frequent and more enthusiastic consumers of sexual media (in both mainstream media and pornography, and with an increasing blurring of these), with some arguing that this intensifies their sexist, sexually objectifying, and violence-supportive attitudes. In addition, young women are under increased pressure to exhibit their bodies, to be sexually available to men, and to conform to the narrow and objectifying sexual codes of pornography and prostitution, with young men increasingly invited into the forms of sexual and gender subjectivity which complement these. On the other hand, raunch culture also has brought an increased assertion of young women's sexual desire and agency and a rejection of norms of female sexual passivity and propriety (Stewart, Mischewski, \& Smith, 2000), with positive impacts on young women's vulnerabilities to sexual violence. (Flood, 2019, p. 32)

A study by Braithwaite et al. (2015) found that viewing pornography increased young adults' engagement in risky sexual behaviour, with a higher number of partners and higher incidence of casual sexual encounters. Similarly, Morgan (2011) found that there was a strong association between viewing sexually explicit material and young adults' preference for sexual interactions similar to those viewed in sexually explicit material, such as 'kinky' sex. In her study $50 \%$ of young women and $92 \%$ of young men reported accessing some form of sexually explicit material including online 
videos, photographs and magazines. Morgan also found that higher frequency of viewing sexually explicit material was linked with reduced sexual and relationship satisfaction. With access to and use of sexually explicit material likely to continue to grow, there is a need to ensure that young adults are presented with a counter narrative to the unhelpful and harmful messaging they are being exposed to where they are educated around realistic and typical relationships and sexual encounters (Keene, 2019). Alongside this, there is a need to develop strategies to keep young adults safe and encourage ethical and safe practices through the use of technology and in online spaces (Powell, Scott, \& Henry, 2020; Classification Office, 2020).

In summary, what this section illustrates is that the contemporary social context within which young adults live is a very different landscape from even just a few decades ago (McHugh \& Frieze, 2006). Today, young adults are navigating their own development, identities, and relationships in a context that is fluid and with a more complicated path to successful attainment of adulthood (Konstam, 2019). This is a time when young people find that there is less adult supervision, greater access to and far more relaxed attitudes towards alcohol and drinking, and more open views on sex and relationships. However, young adults have to balance these relaxed attitudes alongside expectations of established gender roles and the pressure to mimic images of successful masculinity or femininity. The last decade has also seen the huge reach and influence of technology, marketing and media images, leaving young people to navigate the competing messages and unrealistic expectations this creates. This will be discussed in more detail in Chapter Two.

\section{ADOPTING THE TERM 'DATING'}

A key requirement for this thesis was to establish a term to represent the establishment, and experience of intimate relationships that occur during young adulthood, to define what I mean by such a term and to establish the relevance of that term for young people in New Zealand. I use the term 'dating' throughout this thesis, for the following reasons.

Dating is a term that originates in the USA and traditionally described a courtship process, as a pre-cursor to a committed, long-term relationship. However, from the beginning of the $20^{\text {th }}$ century the culture and practice around romantic relationships has 
changed and evolved throughout the Western world. The concept of dating began to represent a far less traditional and formal courtship that was no longer carried out in private and under parental supervision, as it had been in the more formal courtship process (Hunter, 2009). More liberal and relaxed attitudes to dating and pre-marital sex emerged during the 1960s, particularly in the USA. The Freedom Movements advanced during the time of the Vietnam War and the rising popularity of rock'n'roll, protest and civil rights movements enabled "individual mobilization through a sense of morality and (in)justice, and social power through social mobilization against deprivation and for survival and identity" (Fuentes \& Gunder Frank, 1989, p. 179).

These included the Women's Movement, which sought to empower women to become agents of their own sexuality and to think of themselves as more than just wives and mothers; "such movements have been credited with changing social values and transforming government institutions" (Weldon, 2002, p. 61). One of the most important aspects of the freedom movements was the recognition of gendered and racial discrimination and the moves towards increasing the safety of women and children in the home.

The women's movement challenged previously taken-for-granted assumptions about the socially constructed nature of male/female relationships and encouraged women to take more active roles in both the public world and in terms of owning their sexuality. (Hunter, 2009, p. 170)

The women's movement was arguably the first time there was collective profile and recognition of violence against women and emerging concerns around violence in other intimate relationships, including dating, as an area that required urgent action and focus (Weldon, 2002). As Flood (2019, p. 381) states, "work to involve men in reducing and preventing men's violence against women came out of the women's movements, and much of this work continues to be based in feminist and women's rights organisations and networks".

During this time young women gained access to new birth control measures such as the Pill, and as the restrictions of previous eras faded, the taboo of sex before marriage dissipated. This was a time when "young people extolled the benefits of free love - that is, making love without marriage or long-term commitments" (Lindop \& Goldstein, 
2009, p. 64). The sexual revolution redefined sexual activity and sex was no longer viewed as an enactment of marital intimacy for primarily reproductive purposes, but as a choice and right of both men and women to enjoy inside and outside the confines of marriage (Risman \& Schwartz, 2002). However women and feminists remained concerned about the right to be safe from violence, while enjoying the benefits of sexual freedom. This is a particularly pertinent point in that while there appeared to be increasingly relaxed attitudes and behaviours in relation to sexual practices, these were more beneficial for men. Women were still often victims of abuse and still used to fulfil male sexual desires and provide gratification to men (Weldon, 2002).

However, these freedom movements led to a common dating culture for young adults where they could freely engage in more casual relationships or 'hook-ups'. Hook-up is a relatively recent term that has featured in the literature and across popular culture since the early 2000s (Garcia et al., 2019). It refers to young adults engaging in "spontaneous sexual encounters, with or without sexual intercourse, between two individuals with no prior romantic relationship" meaning that the current dating practice is a continuum that can range in intensity and commitment from casual and non-committed relationships to relationships with a long-term partner (Littleton, Tabernik, Canales, \& Backstrom, 2009, p. 793).

The [sexual] revolutionary principle that divorced the right to sexual pleasure from marriage (at least for adults) is no longer controversial; it goes unchallenged by nearly everyone but the most conservative of religious fundamentalists. (Risman \& Schwartz, 2002, p. 21)

The 'hook-up' culture and more relaxed attitudes to casual sex changed practices around the more formal processes of dating. The traditional 'process' of dating is not a wholly relevant concept in the New Zealand context - where people go out on a series of dates in a more traditional monogamous context (Beres, Terry, Senn, \& Ross, 2019). However, across the Western world the concept of 'dating' resonates with young adults as a term that represents a range of romantic and sexual interactions from hookups that last more than just one encounter, through to relationships or pairings where there is some level of exclusivity. 
The term dating is relatable across jurisdictions, including New Zealand. While this may be due to the influence of media and television programmes from the USA, or what Eggleston (2000) terms “Americanism”, the term is also used in other New Zealand studies, particularly those that focus on violence in adolescent and young adult relationships, such as Beres et al. (2019); Jackson (2002); and Appleton-Dyer, Soupen and Edirisuriya (2017).

Therefore the term 'dating' is applied broadly in this study to refer to never married 18-25 year olds in a relationship of three months or more. This allows for a range of degrees of relationship seriousness to be included, and is not bound by other variables such as whether the couple are living together.

\section{DEFINING DATING VIOLENCE}

This chapter opened with an account of Sophie Elliott's murder by her ex-boyfriend. This set the context for this thesis which is grounded in a need to understand how the current social environment in New Zealand contributes to the experience of dating violence for young adults.

Growing research has unearthed the scale of dating and courtship violence, previously believed to be uncommon and insignificant for young adults. This body of research demonstrates that there are commonalities with other forms of partner violence, although continued research into understanding the continuum of dating relationships (casual to more serious relationships) may shed light on the nature and extent of violence within different stages of relationship establishment and commitment (Zweig, Yahner, Dank, \& Lachman, 2014). Dating violence warrants particular attention in the intimate partner violence literature (Lewis \& Fremouw, 2001).

The literature review in Chapter Two highlights that, although research has grown substantially since the 1980 s, there is still limited research on dating violence and almost no specific studies in New Zealand that focus on young adulthood and dating relationships. I saw gaps in the literature, particularly in the New Zealand context, and identified a need to broaden our understanding of this age group in the dating context. I felt that is was important to understand the roots of dating violence as a concept and to seek to conceptualise it in a more meaningful way that could lead to more robust approaches to interventions and prevention strategies. It is critical that effective 
strategies are developed if we are to prevent and intervene in such violence. This requires a strong evidence-base to be utilised in the development of interventions that are tested and evaluated in the social and cultural context that they are implemented (Peterson, et al., 2018).

To do this requires defining what is meant when measuring and discussing dating violence. However, there is a lack of consistency in terms and definitions across the literature, which causes issues in understanding the complexities of violence and aggressive behaviours. The most straightforward definition of dating violence focuses on physical acts of violence, however research suggests this limited focus does not capture the full complexity and experience of other types of violent and controlling behaviour (Shorey et al., 2008). For example, studies such as Anderson (2008) show that psychological violence is more common than physical violence, often precedes physical violence or is used in conjunction with it, and can have longer lasting impacts on victims when compared to those who experienced physical violence only.

Therefore in this study the term 'dating violence' includes any young adult currently dating or dating in the previous 12 months who experienced physical, psychological and/or sexual violence in their relationship (as a victim and/or a perpetrator). This definition of dating violence is relatively inclusive in that it encapsulates a wide range of behaviours that extends beyond just physical violence, and violence born out of gendered norms and beliefs. This is in line with a body of literature on dating violence that has demonstrated the prevalence of non-violent abuse and the severe impact it can have on victims, both male and female (Anderson, 2008; Belknap \& Melton, 2005).

The inclusion of this broad definition could be met with criticism, particularly in what is meant by the term "violence," how impact and harm are defined, and how this broad definition could influence the construction of dating violence and the interpretation of results. The definition in this thesis is intentionally broad to include physical and nonphysical violence, which are measured through the use of the Revised Conflict Tactics Scale (CTS2; Straus et al., 1996). As discussed in Chapter Two, the limitations of the CTS2 are well documented including the impact of certain acts being defined as violent, the lack of context within which these act of violence are experienced, and that acts resulting from arguments or conflict are treated as similar to acts that occur in a wider pattern of control (for example, see Ackerman (2018); Coker, Follingstad, Bush 
and Fisher (2016); and Enander (2011)). Therefore it has been asserted by some that the true nature of IPV is not captured though this scale (Kimmel, 2002).

However, the use of the CTS2 and the application of a broad definition of dating violence in this thesis was a conscious decision so as to be able to replicate Johnson's typology protocol (see Chapter Four). Therefore, as a way to mediate and build on the documented limitations of this scale, I engaged with a wide body of literature that discussed the importance of focusing on the multiple contexts and impacts of dating violence. I also applied a mixed method approach to provide a more nuanced explanation of violence across dating relationships, one that was not focused on the counts of certain acts, but to understand the context that the violence occurred and the social and cultural systems that the violence was situated in.

Taking this mixed-method approach, and focusing more on the qualitative analysis, enabled me to address the potential limitations of using the CTS2. This approach served to extend and strengthen the data by providing a fuller and more adequate explanation of the motives, context and impacts of dating violence in this study, within the acknowledgement of how different scales, sample and ideological perspectives can lead to different constructions of dating violence (see Chapter Two for more discussion on explaining the differences in the role of gender).

\section{DATING VIOLENCE - ITS INHERENT LINKS TO INTIMATE PARTNER VIOLENCE}

To frame my understanding of dating violence, I set out to investigate the roots of our awareness of violence in intimate relationships more generally. During the 1970s the women's movement shone a light on the issue of the gendered nature of violence, identifying the high prevalence of male perpetration of violence against women and emphasising the importance of understanding violence against women in intimate relationships (Jackson, 1998a).

It is women's movements that first articulate the issue of violence against women and press for recognition of it as a public problem, one that requires state action. Although in most places it is recognized that rape and wife beating do occur, it takes women's movements to publicize the extent of these problems 
and to make the case for public responsibility for protecting women's human rights. (Weldon, 2002, p. 64)

Proponents of the feminist or gendered model proposed that the cause of violence in intimate relationships was gender inequality and the use of male authority and domination through coercive control (Day \& Bowen, 2015; Stark, 2007; Dutton \& Nicholls, 2005; Dobash \& Dobash, 2004). Understanding gender as a social construct enabled recognition of how men's violence against women is socially created, disputing notions of biological determinism (Butler, 1988). In the late 1980s Alice Eagly (1987) published on the social role theory of gender, in which she stated that men and women adhere to socially prescribed gender roles that are recreated, reinforced, and reproduced through group consensus and are entrenched in social structures. For example, men are traditionally viewed as the providers or breadwinners while women adopt the role of nurturer and homemaker. Gendered roles define how men and women should act, and the associated traits that support them to successfully conform to these roles are culturally defined and as such vary across different societies and geographical locations (Barnett, Hale, \& Sligar, 2017).

Inherent to socially constructed gender norms are constructions of masculinity and femininity, and the performance of gender. This notion of performativity was described by Judith Butler as a mechanism through which "ontological effects are founded" (Butler, 1994, p. 33).

$$
\begin{aligned}
& \text { It seems fair to say that certain kinds of acts are usually interpreted as } \\
& \text { expressive of a gender core or identity, and that these acts either conform to an } \\
& \text { expected gender identity or contest that expectation in some way. (Butler, 1988, } \\
& \text { p. 527) }
\end{aligned}
$$

Those who readily comply with strong gendered beliefs strongly condemn those who are viewed as violating these gender norms and support their punishment (Griffin, Szmigin, Bengry-Howell, Hackley, \& Mistral, 2013). Therefore it is fear of the negative response to non-conformity that motivates individuals to act according to these expectations, where a deviation is perceived as undesirable and unnatural.

The constructs of masculinity and femininity are complex and influenced by factors such as age, ethnicity, and social class (Connell, 2002). This creates space for multiple 
constructions of masculinities (Jewkes, Flood, \& Lang, 2015). It is theorised that there is an inherent ordering deeming some forms of masculinity more preferable than others in the enactment of manhood (Messerschmidt, 2018). The existence of this 'gender order' led to the concept of "hegemonic masculinity", originally developed by Connell $(1987 ; 1995)$ over three decades ago. The most approved state of masculinity, hegemonic masculinity, is typically idealised in contemporary Western settings as strong, dominant, heterosexual, white and with a high economic standing (Eagly, 1987).

Hegemonic masculinity was understood as the pattern of practice (i.e., things done, not just a set of role expectations or an identity) that allowed men's dominance over women to continue... Hegemonic masculinity was not assumed to be normal in the statistical sense; only a minority of men might enact it. But it was certainly normative. It embodied the currently most honoured way of being a man, it required all other men to position themselves in relation to it, and it ideologically legitimated the global subordination of women to men. (Connell \& Messerschmidt, 2005, p. 832)

While hegemonic masculinity acts to exclude and oppress women, it is also acknowledged that not all men are able to, or choose to, conform to this view of idealised manhood meaning that both men and women are impacted by these hierarchies of masculinities, however it is the lower social status ascribed to feminine norms that are particularly negative and harmful to women (Our Watch, 2017). Where hegemonic masculinity acts as a cultural norm, hook-up culture and dating relationships can become vehicles for young men to assert their status, and relationships with young women can support and reinforce young men's masculinity in their peer groups (Jewkes et al., 2015).

Hooking-up is a way that guys communicate with other guys - it's about homosociality. It's a way that guys compete with each other, establish a pecking order of cool studliness, an attempt to move up in their rankings. (Kimmel, 2012, p. 207)

Performing hegemonic masculinity becomes dependent on the subordination of other groups of people and for this reason it is associated with negative and damaging 
behaviours, such as when men are violent, unable to control their temper or are overly competitive. This does not mean that men are inherently violent but that some men enact dominant expressions such as aggression, violence and control to maintain their performance of hegemonic masculinity (Jewkes et al., 2015; Messner, 1990).

In the context of intimate relationships, the display of hegemonic masculinity is implicated in the use of violence. When both partners are expected to perform their assigned gender roles, women are placed in a subordinate, often dependent, position. Some men holding these beliefs feel an inherent need to exercise power and maintain control in intimate relationships, particularly if their dominance is being threatened and they feel they are failing to live up to heteronormative standards (Brown, James, \& Taylor, 2010).

However, other researchers argue that the causes of violence must be viewed through a gender-neutral lens (Moffitt, Krueger, Caspi, \& Fagan, 2000). Family conflict theorists argue that gender is not strongly associated with violence between intimate partners, maintaining that factors such as personality or ability to resolve conflict are better predictors of perpetration and victimisation (Conger, Cui, Bryant, \& Elder, 2001).

Findings of gender symmetry raise questions about the role of gender in the perpetration of intimate partner violence. The gender debate has left the research community asking why there are such stark differences in findings on the role of gender, which have challenged the gender-sensitive position (Enander, 2011).

This debate on the role of gender is more fully explored in Chapter Two, however this section provides the basis for understanding the complex and sometimes opposing theories of the role of gender in victimisation and perpetration in intimate relationships and the implications in applying the IPV literature to dating violence. These debates and analysis informed the approach to this thesis in understanding dating violence.

The previous sections highlight that young adulthood is a specific developmental stage encased in the current social climate, suggesting that research into dating violence may require a specific framework or focus that is grounded in an understanding of the socio-cultural factors characterising this time in history. Writing within the contemporary Australian context, Michael Flood (2019) has shown how violence is an 
accepted feature of 'normal' intimate and sexual relationships for young people. As he explains it:

...for many young people, sexual harassment is pervasive, male aggression is expected and normalised, there is constant pressure among boys to behave in sexually aggressive ways, girls are routinely objectified, there is a sexual double standard, and girls are pressured to accommodate male 'needs' and desires. (Flood, 2019, p. 17)

This thesis on dating violence is situated in the strong, although sometimes contradictory, body of IPV research, interwoven with an understanding of the unique developmental stage of young adulthood, in the context of the current social culture. This foundation of evidence and context will be developed and tested throughout this thesis as the understanding of the specific nature of dating violence in the current social context is explored. A result of drawing on the IPV literature, in the absence of specific dating violence evidence, means that the term intimate partner violence (IPV) is used throughout this thesis. The World Health Organisation defines IPV:

...as any behaviour within an intimate relationship that causes physical, psychological or sexual harm to those in the relationship... and occurs in all settings and among all socioeconomic, religious and cultural groups. The overwhelming global burden of IPV is borne by women. (World Health Organisation, 2012)

This definition is inclusive and provides a strong framing for understanding the scale and nature of dating violence where there is an absence of specific research and frameworks. However, where research is specifically based on dating violence, this term will be used. 


\section{RESEARCH AIMS AND RESEARCH QUESTIONS}

The overall aim of this research is to measure the level of violence experienced by young adults in dating relationships in New Zealand and to explore the validity of a typology of violence by replicating a specific methodology through a quantitative online survey. From this I aim to explore the utility of the typology in understanding the complexity of experiences of dating violence to determine whether this typology provides a valid framework to better understand the different experiences of violence in dating relationships.

An additional area of investigation was to establish what 'dating' means for young New Zealanders and to understand how the context of New Zealand shapes the experiences of dating for young adults as they move into more serious relationships.

My specific research questions are:

1. What does 'dating' mean for young New Zealanders and how is this constructed within the current context of New Zealand?

2. What is the level of violence experienced by young adults in dating relationships in New Zealand?

3. What is the distribution of the types of violence that occur in dating relationships when replicating Johnson's (2008) typology?

4. What is the utility of the typology in understanding the complexity of dating violence experiences?

\section{THESIS FORMAT}

The following chapters describe the journey to understanding more about dating violence and answering the research questions above.

Chapter Two provides the foundation for understanding the nature and prevalence of violence and the debates that surround the issue, most notably the gendered nature of violence. This chapter builds a case for developing a framework for understanding IPV and dating violence that captures the complexity and dynamics of the issue, most significantly through the concept of typological research. 
Chapter Three focuses on a significant typological framework that has been developed in the USA in an attempt to reconcile the differences between the role of gender in the perpetration and victimisation of dating violence and which forms the platform for the analysis in this thesis.

Chapter Four describes the methodology used to frame, capture and analyse the research questions. This includes a description of the use of mixed method research using key informant interviews, a quantitative survey and a qualitative study.

Chapters Five through Nine discuss the findings from this study. Chapter Five details the quantitative descriptive findings from the online survey and provides detail and comparisons on the reported levels of dating violence for this sample of young adults in New Zealand. Chapter Six presents a more focused quantitative analysis to replicate a typology of violence for this sample.

The main focus of this thesis is to validate this typology through qualitative investigation, but first it was important to locate the experiences of dating and violence in the context of the current social landscape. Therefore Chapter Seven provides a rich qualitative description of the current social context that young people described throughout their interviews. Chapter Eight and Chapter Nine then focus on the way violence is described, made sense of and how it is experienced by the young adults who were interviewed for this thesis, and investigates the validity of typological categories based on their narratives.

Chapter Ten provides a synthesis of the findings across this thesis, weaving together all the strands of data including the literature, survey and interviews.

The final chapter, Chapter Eleven, provides concluding thoughts and closes with recommendations built from this analysis, the findings of previous research, and from the insight and reflections of the young people themselves. These recommendations provide actionable insights that can and should be considered in future policy design. 


\title{
Chapter TWo - DAting Violence, A LITTLE-KNOWN SOCIAL PROBLEM WITH BIG IMPLICATIONS
}

\author{
It appears that violence is a common, albeit neglected, aspect of pre-marital \\ heterosexual interaction. (Makepeace, 1981, p. 100)
}

In this chapter I first focus on the concept of dating violence and its prevalence and then move into an analysis of the gender debate that has dominated the literature on intimate relationships and violence. I then progress to argue for the need to consider theoretical and empirical evidence that focuses on understanding the complexities and dynamics of violence in dating relationships, most significantly the concept of typological research.

The body of research on violent and aggressive behaviours in intimate relationships grew significantly from the 1970s, predominantly in the USA, as the Freedom Movements advanced, with the focus on married women's experiences of violence by their partners. During this time dating violence was believed to be rare and of less significance than violence in marital relationships. However, in a pioneering study, explained later in this chapter, Makepeace (1981) uncovered "a major hidden social problem" (p. 100) in that dating couples, particularly university students and young people in general, did in fact experience high rates of physical abuse (Lewis \& Fremouw, 2001). Makepeace's work led to a number of dating violence studies that quickly supported his findings and in some studies it was reported that dating violence was actually more common than violence experienced in married relationships (Dardis, Dixon, Edwards, \& Turchik, 2015).

In an international dating violence study across 31 university samples in 16 countries, Straus (2004) reported rates of physical assault that ranged between $17 \%$ and $45 \%$ in the previous 12 months. Other studies expanded the definition of dating violence to include psychological violence and found rates as high as $87 \%$ in samples of university students (Harned, 2001). A study by Kaura \& Allen (2004) also demonstrated that once violence was initiated in dating relationships it was likely to increase in frequency and intensity as the relationship continued. 
Studies have consistently shown the negative impacts from experiencing violent and harmful dating relationships with dating violence now viewed as a major public health issue (Fanslow, 2017). Negative consequences include mental health issues such as anxiety and depression; the adoption of negative coping strategies such as substance abuse; and physical impacts including injury (Shorey et al., 2008). For these reasons, young adulthood is a critical period for intervention as long-term intimate relationships are established and coping strategies are developed.

\section{PREVALENCE OF VIOLENCE IN DATING RELATIONSHIPS}

A significant focus of dating violence research has been on assessing the prevalence of violence (Gallaty \& Zimmer-Gembeck, 2008). Before presenting some findings on this aspect, I should note that across the dating violence literature, as with IPV studies, there is ongoing and sometimes 'hostile' debate around the definitions of violence (narrow or broad inclusion), the ways to gather and measure data on the prevalence of IPV (e.g., surveys, crime data, etc) and who data is collected from (e.g., samples of women only) (Flood, 2019).

As for other IPV studies, the different methods, samples and ideological perspectives likely influence the range of findings in the dating violence literature (McHugh, Livingston, \& Ford, 2005). These issues are discussed later in this chapter, however this section on prevalence should be read with caution, as across most of these studies findings are not directly comparable due to the use of different samples, tools and analysis techniques. However, the range of studies on dating violence continues to build a strong picture of the commonly experienced scale and nature of violence in dating relationships that can cause a range of negative impacts on young adults.

The next section highlights some key prevalence studies of young adults, internationally and from New Zealand.

\section{INTERNATIONAL STUDIES}

The majority of research on dating violence has been conducted in the USA, primarily looking at heterosexual relationships and focusing on college (university) students (Jackson, 1998a). 
As mentioned in the introduction to this chapter, the first substantial dating violence study by James Makepeace, published in 1981, involved a study of 202 male and female university students from a Mid-western state university. He reported that $21 \%$ of the college students engaged in or sustained physical violence within a dating relationship. Makepeace (1981) also reported that nearly two-thirds (61.5\%) of students knew someone who had been a victim of dating violence.

In 1983 Bernard and Bernard reported that $30 \%$ of college students they surveyed were involved in dating violence (as either perpetrators or victims). In 1991, White and Koss (1991) reported slightly higher figures: $37 \%$ of college males and 35\% of college females reported inflicting physical aggression to some degree against a dating partner, while $39 \%$ of males and $32 \%$ of female college students reported having sustained some form of physical dating violence.

A surge of research in the early 2000s continued to report high rates of violence in dating relationships. Harned (2002) found $22 \%$ of women and $21 \%$ of men experienced physical aggression in intimate dating relationships. Katz, Washington Kuffel and Coblentz (2002) also reported that between $33 \%$ and $47 \%$ of undergraduate students experienced physical violence in a dating relationship. In both these studies perpetration occurred at similar rates for both male and female participants. Harned's study (2002) also explored other types of dating violence and found that $39 \%$ of women and $30 \%$ of men reported sexual victimisation, and the majority, $82 \%$ of women and $97 \%$ of men, experienced psychological aggression in dating relationships.

More recent research in the USA continues to show high rates of violence experienced by young adults. In a study by Shorey et al. (2010) of 196 male and female undergraduate students, participants completed a survey of relationship aggression and motivations. Results from the study found that $31.1 \%$ reported experiencing physical violence, $23.8 \%$ reported sexual violence, and nearly four in five $(78.8 \%)$ experienced psychological aggression.

Similarly, an online survey of 540 male and female college students aged 18-25 years from a Mid-western university found high rates of dating violence (Marganski \& Melander, 2018). Overall 53.2\% of respondents reported psychological aggression, $23.1 \%$ reported physical violence and $17.4 \%$ reported sexual aggression. 
While the majority of dating violence studies have been conducted in the USA, studies elsewhere also reveal a high prevalence of dating violence. In a study of 166 undergraduate male and female students in Canada, $45.8 \%$ reported experiencing physical violence in their current or most recent dating relationship (Pedersen \& Thomas, 1992). Likewise, Brownridge (2006) asked 120 female students across three Canadian universities about their experience of physical and dating violence. More than a quarter $(26.9 \%)$ of the female students experienced physical assault while $36.4 \%$ experienced sexual coercion. More recently, a further Canadian study asked 34,039 students about their experience of physical assault and verbal threats. Twenty-one percent of students reported they had been violently victimised while $8 \%$ reported that they had been sexually victimised (Daigle, Johnston, Azimi, \& Felix, 2019)

In a study of 7,960 young Mexican adults, aged 11-24 years, 9.4\% of females and $8.6 \%$ of males reported experiencing psychological violence. Almost $10 \%$ of females (9.9\%) and $22.7 \%$ of males reported physical violence (Rivera-Rivera, Allen-Leigh, Rodriguez-Ortega, Chavez-Ayala, \& Lazcano-Ponce, 2007).

In other jurisdictions, a nationally representative sample of 2,250 Swedish women and men aged 15-23 years old answered questions on their experience of emotional, physical and sexual abuse. Emotional abuse was the most commonly experienced, affecting $33 \%$ of women and $18 \%$ of men. Eighteen percent of women and $27 \%$ of men reported physical abuse and $14 \%$ of women and $4.7 \%$ of men reported sexual abuse (Danielsson, Blom, Nilses, Heimer, \& Hogberg, 2009). Similar rates of dating violence were reported in a sample of 4,667 Portuguese young people aged 13-29 years where $13.4 \%$ of participants reported at least one act of physical abuse in the previous year, with 19.5\% reporting emotional abuse (Machado, Caridade, \& Martins, 2010).

In China, a study of 1,015 college students found that more than half reported experiencing psychological violence (58.1\%) while a quarter of students reported experiencing physical violence (25.6\%) (Wang, 2009). More recently a study across a number of Chinese universities found similar rates of dating violence. Across Beijing, Shanghai and Hong Kong 71.6\% of 3,388 students surveyed reported they had perpetrated psychological aggression, $47.7 \%$ reported perpetrating physical violence and just under one-fifth reported perpetrating sexual violence (17.5\%) (Chan, 2012). 


\section{NeW ZEALAND STUdies}

In New Zealand there is a paucity of research into the experience of young adults and violence in dating relationships (Cale et al., 2016). Research that does look at those aged between 18-25 years is generally a sub-sample of a larger population study where the relationship type is unknown, is not reported or the research has focused on younger adolescent (12-18 years old) dating relationships (for example, Fanslow \& Robinson, 2004; Jackson, 2002). However, patterns and trends can still be distinguished from the various studies undertaken in New Zealand.

One significant study is the Dunedin Multidisciplinary Health and Development Study (the Dunedin Study). The Dunedin Study is longitudinal and comprises a birth cohort living in the Dunedin area born between 1 April 1972 and 31 March 1973. Dunedin is a small city in the South Island of New Zealand. Data were collected from the cohort at varying ages from their early and adolescent years through to adulthood. At age 21, 944 of the cohort responded to questions about their intimate relationships (462 women and $482 \mathrm{men}$ ), and $83 \%$ reported having been in a romantic relationship in the previous 12 months. Not all of the 944 were in dating relationships: 71\% reported that they were in a dating relationship, $26 \%$ were in a cohabiting relationship and 3\% were married (Magdol et al., 1997). Findings from the Dunedin Study showed that $21 \%$ of couples who reported being in a dating relationship said that they had perpetrated physical partner violence. Rates were higher for those who were cohabiting, with $48 \%$ reporting that they had perpetrated partner violence. Those aged 21 who had young children were shown to be more likely than other young people in the study to report IPV (some of these couples were in marital relationships). Young mothers were twice as likely to be abused by their partner compared to women who did not have children. Likewise, men who reported being a father were three times more likely to report perpetrating violence towards their partner when compared to men who were not fathers (Moffitt \& Caspi, 1999).

New Zealand's Crime and Safety Survey is a nationally representative face-to-face surveys involving close to 7,000 respondents. Four surveys have been conducted to date, in 2006, 2009, 2014, and 2018. The latest survey was carried out nationwide between March and October 2018, with a total sample of 8,030 New Zealanders over the age of 15 years. It included a self-completion section on psychological, physical 
and sexual violence by a current partner where respondents were asked to describe the most recent incident of abuse. Preliminary results reported that 16 percent of adults experienced one or more incidents of partner violence at some point during their lives with the figure for women being twice that for men (21\% of women compared to $10 \%$ of men) (Ministry of Justice, 2018). No further breakdown by age or relationship status is currently available from the 2018 survey but results from the third New Zealand National Survey of Crime Victims (Ministry of Justice, 2015) found that people aged 20-29 were significantly more likely than any other age group to experience IPV: Thirteen percent of this age group reported violent interpersonal crime by an intimate partner in the previous 12 months (during 2013). This was nearly three times higher than the New Zealand average of 5\%. The lifetime experience of partner violence was also somewhat higher for those aged 20-29 years old at 24.5\% compared to $20.3 \%$ of all adults. Young women were almost twice as likely as young men to report experiencing partner violence over their lifetime (30.3\% and $17.8 \%$ respectively).

One of the few analyses that had a specific focus on dating violence involving a sample of New Zealand young adults was the International Dating Violence Study 2001-2005 (Straus, 2004). This study was initiated in 2000 by Murray Straus and colleagues across 32 countries, including New Zealand. The survey-based study was administered to university students, with 149 New Zealand students taking part. Findings showed that $26.6 \%$ of New Zealand students had physically assaulted a dating partner.

Similar rates were found in a study of high school-aged male and female students living in Auckland, New Zealand. Jackson (1998b) conducted an anonymous 50-item questionnaire with 201 female and 176 male students aged between 16 and 18 years. Jackson found high rates of abuse were experienced in dating relationships, with $81.5 \%$ of female and $76.3 \%$ of male participants reporting experiencing emotional abuse, $17.5 \%$ and $13.3 \%$ experiencing physical abuse and $65.5 \%$ of females and $47.4 \%$ of men experiencing sexually abusive behaviour.

This section has demonstrated many young adults experience forms of violence at alarmingly high rates. The wealth of previous research shows that dating violence is widely experienced and this includes a range of non-violent and violent tactics in the context of dating relationships. These studies also demonstrate that for a large majority 
of young adults this is not in isolation from other forms of violence (O'Leary \& Maiuro, 2001). The extensive experience of violence found across the large body of studies based in the USA is also supported by data from New Zealand-based samples of young adults. These findings support the need to focus on understanding more about the nature of dating violence to inform prevention and education activities to reduce and end such violence. This includes understanding the significance of the role of gender in the experience of dating violence.

\section{GENDER SYMMETRY - THE GREAT CONTROVERSY}

Following on from Makepeace's (1981) study, the body of dating violence research grew and showed that young adults were experiencing high rates of violence in dating relationships and that women were as likely as men to perpetrate some forms of dating violence (for example Robertson \& Murachver, 2007b; Scott \& Straus, 2007; Capaldi $\&$ Owen, 2001). Findings of gender symmetry raised questions about the role of gender in the perpetration of IPV.

In a sample from the Longitudinal Oregon Youth Study, 159 young adult males at risk of delinquency and their female partners were asked about IPV as part of the Couples Study (males were aged 20-24 years at the time of this data collection wave). Findings from this dyadic-response study found that women acknowledged higher rates of perpetration of violence than men (43\% vs. 34\%) (Capaldi \& Owen, 2001). Douglas and Straus (2006) also found that, among dating couples across 17 countries, females admitted assaulting their partners more often than did males (30\% vs. $24.2 \%$ ).

Scott and Straus (2007) looked at minor and severe self-reported perpetration of violence by university students and found female respondents reported similar or higher rates across abuse categories when compared to male respondents. Of note, $15 \%$ of females reported perpetrating severe forms of violence compared to $11 \%$ of men. Female participants also reported higher rates of severe sexual coercion perpetration than male respondents (11\% and $7 \%$ respectively).

In a New Zealand-based study, Robertson and Murachver (2007a) found similar rates of experiencing violence and its impacts for both men and women in a sample that included students, general population and incarcerated people. In fact, their analysis 
found that women were more likely to perpetrate violence towards their male partner and men were more likely to be victims of IPV.

This growing body of evidence of women's use of violence fuelled the debate between two opposing perspectives of IPV and dating violence, the feminist-based perspective and the family conflict perspective (Melton \& Belknap, 2003). The growing evidence of gender symmetry found by family conflict researchers was in stark contrast to the previous feminist-based analysis that clearly identified women as victims of their male partner's violence.

Statistics on marital violence were first collected in the 1970s and 1980s by feminists who worked directly with, or interviewed, women who had experienced severe violence at the hands of their intimate partner (Frieze, 2005). Data on the scale and nature of such violence in New Zealand was presented by agencies like Women's Refuge. The trend shown by these statistics was mirrored in other jurisdictions including the USA and the United Kingdom, where men's violence towards women was reported at higher rates (United Nations Human Rights Council, 2011).

The women's movements and feminist-based research brought to light the importance of understanding violence against women in intimate relationships, who some researchers initially labelled as 'battered women'. Battering or wife abuse was framed around men's need for power and control over their partner. This patriarchal perspective of IPV is grounded in the gendered nature of violence and holds that violence is asymmetrical, that is that men commit most of the violence in intimate relationships as a result of patriarchal values (Bates, 2016).

Traditionally, IPV has been framed from a patriarchal perspective, whereby men's need to maintain power and control in society and at home is at the root of IPV; men systematically and intentionally use violence to maintain a power system in which men are dominant and women are subordinate. (Hines \& Douglas, 2010, p. 37)

Feminist-based researchers posit that violence against women is situated in the concept of coercive control. Some theorise that it creates the foundation for men's use of physical violence in an intimate relationship in order to maintain and reinforce power 
and domination within beliefs that manifest in toxic masculinity (Pence \& Paymar, 1993). Coercion is defined by Evan Stark as:

"the use of force or threats to compel or dispel a particular response” (p. 228), while control refers to "structural forms of deprivation, exploitation, and command that compel obedience indirectly" (p. 229). When coercion and control occur together, he argues, the result is a "condition of unfreedom" ( $p$. 205) that is experienced as entrapment. (Stark \& Hester, 2019)

In an attempt to exert general control a violent man can use a range of tactics, including physical violence, if their partner does not respond to their non-violent tactics and demands (Leone, Johnson, Cohan, \& Lloyd, 2004). In fact, because of the effectiveness of non-violent coercive tactics to gain control and compliance, it is argued that the use of physical violence is not always necessary; across studies a large proportion of coercive control happens in the absence of physical and/or sexual violence (Stark \& Hester, 2019).

Those males who occupy a seat at the patriarchal table are less likely to need 'violence as maintenance' because their elevated position is sustained in legitimate ways.... Direct threat and coercion are hardly necessary in a world where gender relations are entrenched and remarkably self-perpetuating. (Hunnicutt, 2009, pp. 560-1)

Many hundreds of studies have reported the experiences of abused women and the control and domination they experience at the hands of their intimate partner (see Towns, 2007; Adams, Towns, \& Gavey, 1995; Dobash, Dobash, Wilson, \& Daly, 1992), with some women experiencing the most serious negative outcomes. Family conflict researchers have more recently begun to gather experiences of violence from population-based samples using national and community-based studies and often focusing on questions on the use of specific actions that are defined as abuse (Belknap \& Melton, 2005). These studies of IPV, including violent dating relationships, demonstrate women use some forms of violence in intimate relationships at similar rates to that of male partners, in some cases inflicting as much harm as maleperpetrated violence (Archer, 2000; Hamberger, 2005). 
A meta-analysis by John Archer (2000) showed that while both sexes reported perpetrating physical violence, women were actually significantly more likely than men to use one or more acts of physical aggression towards their partner and to report using these acts more frequently than male participants, a stark contrast to previous gender-based research. However, when measures of the consequence of the violence were reviewed, men were more likely to be responsible for more serious outcomes such as injury where medical attention was required. The focus of Archer's metaanalysis was on gender differences in marital and dating relationships and his review examined research published between 1976 and 1997 and included supplementary data sources from police and hospital records. A limitation of Archer's meta-analysis was the focus on physical violence and not other forms of violence, such as sexual assault and coercive control, where higher rates of male perpetration are found (Swan \& Snow, 2006).

Findings from a sample of New Zealand young adults who took part in the International Dating Violence Study 2001-2005 (Straus, 2004) showed that females were more likely to report being physically assaulted by their partner: $29.2 \%$ of females compared to $16.7 \%$ of male respondents. However the injury statistics reversed this gender finding, with $7.1 \%$ of participants reporting causing an injury as a result of dating violence: $8.3 \%$ of males and $6.7 \%$ of females. Of note, all of the injuries reported by the New Zealand sample were classified as minor with no severe injuries being reported for this group.

The gender debate has left the research community asking why there are such stark differences in findings on the role of gender which have challenged the gendersensitive position (Enander, 2011). In a commentary, Amy Holtzworth-Munroe states that while there is a growing volume of work demonstrating women's use of violence:

research focused on female aggression remains quite controversial outside of the ivory tower ... Thus, our new research interests threaten to disrupt the relatively hard-won and uneasy coexistence of advocacy workers and researchers in the area. (Holtzworth-Munroe, 2005, p. 252) 
EXPLAINING THE DIFFERENCES IN THE ROLE OF GENDER - A FOCUS ON METHODS, SAMPLES AND IDEOLOGICAL PERSPECTIVES

Controversy surrounding the role of gender has led researchers to focus on the impact of different methods, samples and ideological perspectives that may influence the range of findings on the role of gender in the study of IPV (McHugh et al., 2005).

\section{SAMPLING FRAMES}

One reason posited for the gender difference found in IPV perpetration between feminist-based researchers and family violence researchers is the different samples from which the data are derived, with different groups included in each study leading to different constructions of IPV (Holtzworth-Munroe, 2005). If the focus of a study is on women in women's shelters then we construct the expression 'wife abuse' where females are victims of their male partners' violent and controlling behaviours. In this case the focus on battered women means that violence by women is not examined or is captured as violence used in self-defence (Robertson \& Murachver, 2007b). In contrast, if the study is focused on young dating adults then alternative constructions of violence are developed and labelled as such (McHugh et al., 2005).

The construction of IPV and findings on the role of gender may also be influenced by the large proportion of research that is conducted in the USA that is largely focused on white college and university students in heterosexual relationships (Jackson, 1999). This population likely differs from both the general population within the Unites States and samples across other countries, therefore the large body of IPV findings may not be representative of relationships in other settings. The focus on university students may also mean that less serious forms of violence that are mutually perpetrated may be over-represented and mean that the more gendered and enduring violence more likely seen in crisis support samples is not captured (Enander, 2011).

... the inherent biases of sample selection slant the results in the direction of whatever type of IPV is most represented in the sampling frame and by the sampling plan. In general, that means that survey results are presented by some scholars as if they apply to all domestic violence, when in fact they are relevant to only situational couple violence, and that agency results that concern the centrality of the power and control dynamic are taken by other scholars to 
apply to all violent couples, when in fact they are relevant only to intimate terrorism and violent resistance. (Johnson, 2017, p. 157)

This limitation is supported by researchers such as Australian Associate Professor of Law Michael Flood (2006) who discussed that it is more likely that mutually perpetrated, less severe violence was reported in student or community-based samples compared to crime victimisation studies or crisis support samples where more serious forms of violence are reported.

\section{METHODS FOR COLLECTING DATA - HOW WE COLLECT INFORMATION}

The methods used to study IPV are another reason that we might see different conclusions on the role of gender in IPV. For example, research carried out in women's shelters has often been conducted using open-ended questions through qualitative methods. These methods produce a rich description of the "unilateral, chronic, serious, and escalating violence perpetrated by men on their intimate partners" (McHugh et al., 2005, p. 328).

Research that finds patterns of gender-symmetry in the experience of violence has often been found in nationally representative samples or samples of university-aged young people using survey methodology which, it has been argued, captures a range of less serious incidents of violence than those captured through clinical and shelter samples (Hamberger, 2005). The survey methods rely on the use of standardised scales where results are categorised by the researchers into violent or non-violent experiences. While both types of data collection methods are important and add to the more general understanding of the experience of IPV, what is also important are the different perspectives on experiences of violence and how each method contributes to the understanding and construction of different aspects of violence (McHugh et al., 2005).

\section{SAMPLING FRAMES - WHO WE TALK TO}

An area of focus in IPV measurement is who data is captured from and their role in the intimate relationship. Often one partner is asked to report on the violence they and their partner experience, however research that has interviewed both partners in the relationship has found that there is significant disagreement about the occurrence of 
violence: when it began, who initiated it and its impact (McHugh et al., 2005). This lack of concordance is seen in both clinical studies and in survey samples.

Researcher such as Lehrner and Allen (2018) have reported that there are differences in the way that men and women report on experiences of violence. There are a number of reasons posited for this lack of concordance between male and female intimate partners. In research using both quantitative and qualitative methods, Currie (1998) found that levels of reported violence depended on the sex of the perpetrator. Both women and men under-reported men's violence towards women while men were more likely to overstate women's violence towards them. Shorey et al., (2008) explain that this pattern of reporting may be due to men's violence being far less acceptable than women's violence, therefore men may be less likely to report their use of violence.

Women may also be more likely to report their use of violence in an intimate relationship because women's violence is viewed by society as less serious, they are less likely to be condemned for their use of violence and women use a lower threshold than men to label their behaviour as violent (Lewis, Travea, \& Fremouw, 2002). Harned (2001) expands on this gendered explanation in the context of victimisation by arguing that men may report higher levels of victimisation as these events run counter to beliefs about feminine gender role conformity, while women tend to under-report men's violence as it conforms to male stereotypes of being dominant and aggressive and is therefore more likely to be normalised or minimised by them. This is especially so for sexual violence where women are less likely to label their experience as sexual violence if it does not follow a typical 'rape script'. Studies have found that women are more resistant to defining their experience as rape when the perpetrator was their boyfriend, there was alcohol or drugs involved and/or there had previously been consensual sex (LeMarie, Oswald, \& Russell, 2016). Women's under-reporting of sexual violence and rape can also be attributed to victim blaming and rape myths that discount allegations of sexual violence. Rape myths are false beliefs that blame victims for their victimisation and reinforce the actions of perpetrators as justified, irrespective of gender (Beres et al., 2019).

New Zealand academic Nicola Gavey talks about the "cultural scaffolding" of rape where constructions of gender and what it means to be feminine or masculine in a relationship produce expectations of behaviour (Gavey, 2019). This can support false 
beliefs that lead to victim blaming and normalise beliefs about men's natural sexual aggression and women's sexual passivity, even when the concept of rape itself is widely condemned by society (Beres et al., 2019). Rape myths can be internalised and prevent victims from identifying their experiences as rape. They can also influence perpetrators by minimising the nature and impacts of their own abusive behaviours, with victim-blaming a common societal response.

Studies by researchers such as Chung (2005) also suggests that young adults who are currently experiencing violence in a relationship are less likely to label this abuse as violence for a range of additional reasons, the most common being the shame victims feel. A second reason is linked to the couple's identity, as acknowledging the violence would position one partner as a 'victim' and erase equality in the relationship. Finally, for female victims, gender scripts place pressure on them to choose a suitable boyfriend. An inability to do this reinforces the belief that violence is the individual's problem and therefore they have failed to successfully achieve femininity (Chung, 2005). It becomes easier to label a relationship as violent once the relationship has ended and their identity is no longer attached to the relationship.

\section{HOW WE DEFINE AND FRAME VIOLENCE}

The people we choose to study, where they are sampled from, what method we use to capture information and who is capturing this information can influence conclusions made across different studies. However, it is not only participants' own representation and labelling of their experience of violence that can impact on findings and our understanding of IPV, but also researchers' own definition, interpretation and social judgement of how they classify different events and experiences (McHugh et al., 2005).

Feminist-based researchers often argue that studies that show women perpetrating violence at similar or higher rates than their male partners result from researchers focusing solely on the act of violence (whether you hit someone or not, once or more). In this context feminist-based researchers argue that the analysis of gender and perpetration ignores the context of the violence, including the motivation (power and control versus self-defence) and the outcome (levels of fear and injury), thus exaggerating women's use of violence (Melton \& Belknap, 2003). Therefore studies 
that simply count acts of violence without accounting for the context within which they occur may inflate the rates of violence that women commit (Shorey et al., 2008). In studies where data is collected on the impact of violence, women tend to be significantly more affected, experiencing greater levels of fear (Borsky, McDonnell, Rimal, \& Turner, 2016), higher levels of injury (Archer, 2000) and more negative mental health outcomes compared to male victims (Leone et al., 2004). This supports approaches to dating violence research that capture the context in which the violence occurs.

In an attempt to capture accurate and reliable rates of IPV, a number of scales and measures have been developed (Dobash \& Dobash, 2004). One of the most prominent tools is the Conflict Tactics Scale (CTS) developed by Murray Straus (1979) to measure a range of types of behaviours used to resolve conflict between intimate partners. Respondents indicate whether they have experienced any of the act-based items in the scale over the previous 12 months (Jackson, 1999). Researchers such as Ackerman (2018); Coker, Follingstad, Bush and Fisher (2016); Enander (2011); and Kimmel (2002) have criticised the CTS, saying it does not capture the true nature of IPV. The main criticism is that the CTS counts acts of conflict, which are hard to interpret without information on the context and circumstance of the violence (Hamberger, 2005). Without data on who initiated the violence and the context of or motivation for the violence researchers fail to capture the intention of the violence and its true impact (Enander, 2011; McHugh \& Frieze, 2006).

The CTS simply counts acts of violence but takes no account of the circumstances under which these acts occur. Who initiates the violence, the relative size and strength of the people involved, and the nature of the relationship all will surely shape the experience of the violence but not the scores on the CTS. Thus, if she pushes him back after being severely beaten, it would be scored one conflict tactic for each. And if she punches him to get him to stop beating their children or pushes him away after he has sexually assaulted her, it would count as one for her and none for him. (Kimmel, 2002, p. 1341)

Despite this criticism, the CTS is reported to have good internal consistency, construct validity, discriminant validity and validity with a variety of populations (Babcock, 
Snead, Bennett, \& Armenti, 2019), and it is still the most widely-used measure of IPV, including in dating violence research (McHugh et al., 2005). The extensive use of the tools does allow some level of comparison across different studies and provides some consistency in how IPV and dating violence are measured.

\section{SOCIAL AND HISTORICAL CONTEXT}

A final element covered here that may impact on the measurement of violence is the social and historical circumstances in which studies are conducted. Makepeace's study on dating violence (referenced earlier) was carried out in the early 1980s, nearly 40 years ago (Makepeace, 1981). Since then many more studies looking at violence in dating relationships across a rapidly changing social context have been conducted. With the more fluid paths into young adulthood described in Chapter One, and the rapid decline of traditional dating in the Western world leading to a growing acceptance of 'hooking-up' and casual sexual encounters often within the situational context of going out on the town and consuming excessive amounts of alcohol (Hamilton \& Armstrong, 2010), it is likely that contemporary young adults are at increased risk of experiencing physical and sexual violence (Noel, Ogle, Maisto, \& Jackson, 2016).

\section{GENDER SYMMETRY IN CONTEXT}

While there are many reasons posited as to why there are differences in the reporting of gender, the studies reporting gender symmetry cannot be discounted. The growing controversy of women's use of violence has led to significant debate on its equivalence to male violence and poses the question of whether gender symmetry implicitly means that the motivations and impacts of violence are the same for women as men (Enander, 2011). Terms such as 'symmetry' and 'mutual' imply that there is similar tendency and socialisation for men and women to use violence and also imply that both men and women have a similar ability to cause injury, inflict fear and to enact control towards their partner (Hamberger, 2005).

Does gender symmetry mean that women hit men as often as men hit women?

Or does it mean that an equal number of men and women hit each other? Does gender symmetry refer to men's and women's motivations for such violence, or does it mean that the consequences of it are symmetrical? These questions are 
often lumped together in reviews of literature and meta-analyses (Kimmel, 2002, p. 1335)

As highlighted earlier in this chapter, there are a significant number of studies that support the argument that women commit acts of violence as often, or more often, than men and that the majority of this violence is bi-directional, that is, that both individuals in the relationship are violent towards each other (see Archer, 2000; Straus, 2004; Hamberger, 1997). Data from the New Zealand students who participated in the international dating violence study showed that of the $27 \%$ of young adults who reported experiencing some level of physical violence in the previous 12 months, $63 \%$ reported that both partners were violent in the relationship while only $8.7 \%$ male partners reported that they were the sole perpetrator and $28.2 \%$ of females reported that they were the sole perpetrator in the relationship (Straus, 2004). However, data that reports on the patterns of violence shows a more complex gendered picture with women more likely to experience fear and terror from coercively controlling violence and also being far more likely to be physically injured and experience greater psychological harm as a result of male-perpetrated partner violence (Hamberger, 2005; Holtzworth-Munroe, 2005). This is further explored in the following sections.

Research investigating the differences in motives for and outcomes of violence by men compared to women shows differences in the consequences of violence. Such studies suggest that while there may be mutual violence within relationships, this does not mean that the motives and effects of men's and women's violence are symmetrical (McHugh et al., 2005).

\section{INITIATING VIOLENCE}

One area that may shed more light on the gender symmetry debate is in understanding the patterns of violence and whether there is equal likelihood of men and women initiating violence in the intimate relationship (Hamberger, 2005). It is argued that women's violence should be seen in the context of their own victimisation. This means that female violence is more likely to be in response to men's violence and that men are more likely to introduce violence into the relationship, suggesting that the primary motive of women's violence is self-defence (Swan \& Snow, 2006). This is evidenced by studies that show women's violence is often a response to being victimised or is 
retaliatory in nature (see the next section for a discussion of motives for using violence).

Swan and Snow (2006) carried out a study with 108 women who had recently reported the use of IPV. Nearly all of the participants (102 of the 108 women) reported some form of injury inflicted by their male partner. While female participants reported perpetrating moderate violence, they also reported that they were more likely to be a victim of more severe types of violence including sexual coercion, coercive control and injury. These findings suggest it is important to consider the entirety of the pattern of violence, particularly when coercive controlling violence has the potential for far greater psychological impacts than physical forms of violence (Shorey et al., 2010).

Other studies that support findings that men are more likely to initiate violence in the relationship include DeKerseredy and Schwartz's (1998) study of a sample of Canadian college/university students where they reported that women who were physically aggressive in their dating relationship said that they never initiated violence. Hamberger and Guse (2002) found similar results in a clinical sample of men and women court-ordered to attend domestic violence programmes. Women reported perpetrating physical violence but were less likely than male perpetrators to initiate the violence, therefore their violence could be seen as a response to their partner's actions.

However, there are contradictory findings across a growing body of research where women report that they initiated violence in the relationships. In 1990 Stets and Straus reported that women-initiated violence at the same rate as men. Similarly, in a study of 52 women who were arrested for domestic violence, Hamberger (1997) found that women reported initiating violence with $25 \%$ of arrested women reportedly initiating $100 \%$ of the violent incidents in their relationships. However, rates were still higher for their male partners, who initiated violence $37 \%$ of the time. Hamberger (1997) also found that in $51 \%$ of relationships the male partner was reported to be first to commit physical violence compared to $27.4 \%$ of women, indicating that men were more likely to begin the overall pattern of violence in the relationship. These rates of women's initiation of violence are also likely to be higher than other studies as these women had been arrested for domestic violence, thus producing a biased response to their initiation of violence. 
The findings above suggest that women do sometimes initiate IPV, and often this is reported at equal rates to men. However, work by Hamberger and Guse (2002) found different results. They collected data from a number of settings including women receiving programmes through a domestic violence shelter and men and women attending a court-ordered programme to address their domestic violence offending. When they asked about the proportion of incidents that men and women initiated in a relationship, men were statistically more likely to initiate more frequently across violent events. Men were also more likely to report introducing violence into the relationship when asked who initiated the first ever event of violence (Hamberger, 2005). These findings suggest that the symmetry of violence is questionable when more context is added to the analysis around the events of IPV.

\section{MOTIVATIONS FOR VIOLENCE}

Another area that may shed light on the gender symmetry debate is a focus on the motives for committing violence towards an intimate partner. A number of studies show that there is a diverse range of motives for both male-perpetrated and femaleperpetrated violence (Straus, 2008). Motives that have been identified in the literature include intimidation, control, self-defence, to 'show who's the boss', poor impulse control, poor conflict management and expression of feelings (Hamberger, 2005; Dutton \& Goodman, 2005; Pence \& Paymar, 1993). Within the range of motives there appears to be some overlap in the reasons given by male and female perpetrators, while there also appear to be some commonly reported differences (Holtzworth-Munroe, 2005).

Saunders (1986) found that self-defence was the most common reason for battered women's use of violence (between $31 \%$ and $39 \%$ of women). They also found that $23 \%$ of battered women who reported using minor violence and $32 \%$ of women who used severe violence reported their motive was to "fight back", demonstrating that women's violence is used in response to their male partners' violence and aggression. In the same year Makepeace published a study on college students' motives for violence, asking them to respond about the worst episode of violence they inflicted. Makepeace (1986) reported that women were more likely than men to report selfdefence as their motive ( $36 \%$ and $18 \%$ respectively). 
Likewise, Foshee (1996) conducted a study involving 1,405 high school students in the USA with an average age of 13.9 years using self-administered questionnaires. The study found that females were more likely to report that their use of physical violence was in self-defence, compared to male students.

These studies are consistent with the feminist-based researchers' argument that women's use of violence is a response to their male partner's violence and is therefore motivated by defence of themselves or their children, or retaliation against their abusive partner (Hamberger, 2005; Pence \& Paymar, 1993).

However, other research has begun to show that women self-report that their main motivation for IPV is not self-defence but anger, jealousy, retaliation, control and dominance (Hines \& Douglas, 2010). Dutton and Nicholls (2005) cited a number of studies that showed that there are very few cases where self-defence was the primary motive for women's IPV. A study by Harned (2001) showed that both male and female participants reported the use of violence as a way to get control over the other person, to feel more power and to get their partner's attention. In contrast, motivations that focused on men's use of violence were more likely to include an attempt to exert power and control as a display of hegemonic masculinity, to maintain dominance of their female partner (see the previous section in this chapter for the discussion on gender roles and hegemonic masculinity).

More recently Shorey et al. (2010) carried out a study with 196 undergraduate students looking at the prevalence and motivations for their use of dating violence. Respondents reported a range of 29 motivations at varying levels. One of the specific scales that Shorey et al. (2010) used was the Motivations for Self-Defence Scale. Results showed that female college students were not more likely to use violence in self-defence than male college students, but suggest that male and female students do have different reasons for their use of violence. They reported that the most common motives for men's use of physical aggression (in order of most commonly reported) were to get their partner's attention, jealousy or because it was sexually arousing. The main motives offered by women were because it was sexually arousing, to retaliate for being emotionally hurt or to get their partner's attention, or to show feelings they could not explain in words. 
Similarly, a study of 409 college women found that less than $5 \%$ of women who perpetrated both minor and severe violence did so in self-defence (Leisring, 2013). The motives that were most commonly cited for perpetrating minor and severe physical aggression and emotional abuse were anger, retaliation for emotional hurt, to get their partner's attention, jealousy, or stress.

The findings on motives are mixed, with some research supporting a difference between men and women in their reasons for using violence (Straus, 2008). This difference appears more pronounced in studies of women in crisis support samples compared to analyses of community and student samples. What the studies presented here show is that both men and women report a number of motives for their use of dating violence and that young women's motives for violence are not primarily based on the notion of self-defence and fighting back, although these motives are still reported more frequently by young women than young men.

\section{IMPACT OF THE VIOLENCE}

An area where gender symmetry does not appear to hold as strongly is when investigating the impact of violence against a partner. A significant number of studies have looked at the prevalence and severity of harm resulting from violent relationships (Hamberger, 2005). This research provides general support for the argument that male violence has far more negative impacts than does female violence in intimate relationships when considering severe physical injury, medical treatment, psychological outcomes and fear (Holtzworth-Munroe, 2005; Harned, 2001).

In Archer's (2000) meta-analysis, he found that women perpetrated physical acts of violence in their dating relationship at a higher rate than men. However, he also found that women were significantly more likely to sustain injury inflicted by their male dating partner, with $62 \%$ of those injured being women.

In 2010, a USA research consortium collected data as part of a study on IPV and sexual violence (Black, Basile, Breiding, \& Smith, 2010). In this study of 16,507 adults (9,086 women and 7,421 men), $35.6 \%$ of women and $28.5 \%$ of men reported experiencing physical violence, sexual violence and/or stalking by an intimate partner. Of those who were victims of IPV, $14.8 \%$ of women reported an injury as a result of their partner's violence, compared to just $4 \%$ of male respondents (Black et al., 2010). 
One statistic that shows a clear gendered pattern is intimate partner homicide where statistics show an overwhelming likelihood that men are more likely than women to commit lethal IPV (Dobash \& Dobash, 2004). Data from the New Zealand Family Violence Death Review Committee (NZFVDRC), established in 2008, annual statistics on family violence homicides in New Zealand, shows that between 2009 and 2015 there were 194 family violence deaths, an average of 28 family violence deaths each year. Of these, 92 were coded as IPV (NZFVDRC, 2017). This data shows a clear gendered pattern for those who were offenders and those who were murdered. Of the 92 IPV deaths, three-quarters of the offenders were male (76\%) and $68 \%$ of victims were female (NZFVDRC, 2017).

While physical injury is the more observable outcome from violence there are other negative impacts experienced by those in violent relationships (Hamberger, 2005). One of the defining characteristics of more severe and controlling violence is the ability to instil fear and terror in the victim, which is used to control the victim by forcing them to conform to behaviours that reduce the potential to need to use violence (Hamberger, 2005).

Data indicates that compared to men, women report greater levels of fear towards their violent partner. Black et al. (2011) used data from the National Intimate Partner and Sexual Violence Survey of adults (18 years and over) and found that, of those who experienced physical or sexual violence and/or stalking by an intimate partner, $72.2 \%$ of women reported being fearful compared to just $18.4 \%$ of men who experienced such violence. Sixty-two percent of the female respondents also reported feeling concerned for their safety and $62.6 \%$ reported at least one symptom of post-traumatic stress disorder compared to men who reported $15.7 \%$ and $16.4 \%$ respectively.

However, researchers such as Denise Hines and colleagues state that severe male victimisation has largely been ignored in the study of IPV, resulting in little understanding of the impact of extreme female violence on men (Hines, Brown, \& Dunning, 2007). Using data from 190 men who called a Domestic Abuse Helpline in the USA, Hines et al. (2007) reported that men experienced severe control, manipulation and violence, and as a result reported fear of their violent wives. They concluded it was therefore important to not discount male victims of intimate violence 
and that their experience is potentially serious, causes psychological and physical harm and is valid in its own right.

In summary, the literature above shows that the controversy of gender and whether men and women are equally likely to perpetrate violence remains unresolved (Harned, 2001). However, the literature does highlight that dating violence, and violence in other intimate relationships, needs to be placed in the context of a gendered analysis, which is done through data collection and analysis that places the acts of violence within the context, motivations and consequences of the violence (Jackson, 1998a). Looking at differences in contexts of violence may shed light on gender differences and help in understanding what lies beneath findings of gender symmetry in IPV, particularly when there are gender inequalities that position women at a disadvantage within a male dominated society.

However, findings on gender differences in motive and impacts are inconsistent. Even when there is a focus on the nuanced picture of the nature, motives and impact of violence, in some cases women do report initiating violence, are motivated for reasons other than self-defence and can cause injury and other harms to their male partner.

Therefore, there is a need to acknowledge that women's use of violence is not always a response to being victimised, and to understand the impact of victimisation on men. It is important then that factors for female-perpetrated violence are researched and better understood to be able to address and prevent all types of dating violence (Espinoza \& Warner, 2016). Acknowledging the experiences of both genders means different factors can be understood in the aetiology of dating violence (Ortiz, Shorey, \& Corneliua, 2015). This needs to be done in a sensitive way so as not to minimise and conflate the experiences of women who find themselves in coercively controlling or abusive relationships.

\section{RECONCILING THE DIFFERENCES - AN INCLUSIVE DESCRIPTION OF INTIMATE PARTNER VIOLENCE}

McHugh et al. (2005) suggest that, to date, much of the dating violence and IPV literature has focused on proving a 'single truth' in its explanation, treating those who experience violence as a homogeneous group. Evidence suggest this is not the case, with studies showing high rates of violence for young women and men in dating 
relationships, women reporting similar rates of perpetration and the experience of young men as victims (McHugh \& Frieze, 2006). There is a growing narrative that says we need to view IPV as a multi-faceted, complex and dynamic interaction between intimate partners. A more sophisticated conceptual framework is required that captures the heterogeneous and complex nature of violence in dating and other interpersonal relationships (Lewis \& Fremouw, 2001). A conceptual framework that allows for a more "complex, contextual and layered understanding" of IPV will support us to develop more sophisticated responses to the types of violence that occur in different relationships (McHugh et al., 2005, p. 324).

The need for a more sophisticated approach to conceptualising IPV has led a number of researchers to develop empirically-based theories and models that attempt to reconcile different findings across studies. One approach to this is to apply a typological framework to measuring and understanding violence in intimate relationships. A typology is:

a system of groupings, the members of which are identified by postulating specified attributes that are 'mutually exclusive and collectively exhaustive'. (Encyclopedia Britannica Online, 2014 in Gulliver \& Fanslow, 2015, p. 25)

A number of typologies have been developed across the international IPV literature, for example Dutton, Kaltman, Goodman, Weinfurt and Vankos (2005) and Swan and Snow (2002) developed separate approaches that focused on categorising IPV experiences based on the frequency, impact and severity of the violence.

Other typologies have focused on the classification of male perpetrators. One of the most significant typologies on male perpetrator types was developed by HoltzworthMunroe and Stuart (1994) who assigned four categories of batterers based on three descriptive dimensions - severity of marital violence, generality of violence and psychopathology. The foundational assumption of their typology is that men's violence is not homogeneous, and they conclude that considering different types of offenders is necessary in understanding the complexity of the causes and impacts of violence. This is particularly critical when developing treatment and interventions to address this violence - again supporting the view that a more sophisticated approach to 
understanding the complexities of violence in interpersonal relationships is needed (Holtzworth-Munroe, 2000).

However, one of the most influential typologies in the IPV literature is Michael Johnson's (2008) typology of violence; the origin of which was an attempt to reconcile differences in the gendered nature of IPV. In his first publication on his typology of violence, (Johnson, 1995) argued that disagreement about the role of gender in IPV was the result of researchers looking at different parts of the same phenomenon (Enander, 2011).

My discovery of Johnson's typology as part of the review of literature for this thesis was a pivotal moment and it became the primary focus of how I would frame the experience of dating violence, encapsulating the need for a more complex analysis to inform the gender debate. The next chapter provides more detail on his typology and how it was applied in this study.

\section{CHAPTER SUMMARY}

In this chapter evidence has been presented to show that dating violence is experienced by a large proportion of young people and can have significant negative outcomes. Although there has been a large increase in attention on dating violence since Makepeace's (1981) seminal study, there are still a number of limitations across this body of work that impact on our ability to adequately understand and prevent this type of violence for young people (Lewis \& Fremouw, 2001). More significantly, the dearth of information on dating violence in New Zealand places a substantial limitation on our ability to intervene and respond to it in the context of young people's current experiences and circumstances.

The debate between feminist-based studies which view violence as being embedded in gender and power imbalance, and family violence researchers who are more focused on act-based analyses to explain women's use of violence, is not close to being resolved. This is particularly so in the dating violence literature. The role of gender in dating violence needs to be the focus of future research, particularly to better understand the function of dating violence, who initiates violence, the motives for the violence and the impact of the violence (Lewis \& Fremouw, 2001). A focus on the role of gender can help us to understand more about the women who report using violence 
towards their intimate partner. The lack of focus on women's use of violence limits our understanding of the complexities of violence in dating relationships, which in turn limits the range of responses and interventions that are available to reduce the experience and impact of violence for both young men and women (HoltzworthMunroe, 2005).

The above literature draws us to the need to develop frameworks that reflect a more nuanced and sophisticated understanding of violence in intimate relationships and to move beyond the need to find a singular explanation within a complex interaction of circumstances. Researchers have begun to look at ways to reconcile the differences in findings by looking at context and outcomes within a typological framework. The next chapter will detail Johnson's (2008) typology of violence and how it could usefully be applied in understanding the scale and nature of dating violence in New Zealand. 


\title{
CHAPTER THREE - RECONCILING THE DIFFERENCE, A TYPOLOGICAL APPROACH
}

\author{
Obviously, I think it is essential that we attend to differences among these basic \\ types of intimate partner violence. All of them are dangerous, and all of them \\ require our attention in prevention and intervention, but they develop \\ differently, have different implications for relationships, and will require \\ different strategies to address them as social and personal problems. (Johnson, \\ 2009, p. 294)
}

In this chapter I discuss how Johnson developed his typology and I review a number of studies that have applied it. From this I argue Johnson's typology is a valid approach to providing a more sophisticated analysis of IPV and look at the application of Johnson's approach as a basis for further research into understanding the dynamic experiences of violence in dating relationships.

As detailed in the previous chapter, many IPV studies conclude that females are as violent as their intimate male partners and in a majority of cases this violence was bidirectional, sparking claims that there is gender symmetry in the perpetration of violence in intimate relationships (Douglas \& Straus, 2006). These findings were met with considerable criticism from researchers including feminist-based researchers who argued that these findings of gender symmetry were misleading and did not account for the context and motivations of violence, and the intent and harm caused by the perpetrator (McHugh et al., 2005).

My literature review led me to discover a typology of violence developed by Michael Johnson (2008), which aimed to reconcile the differences between research that demonstrated male perpetrated violence embedded in gender and power imbalance versus research that suggests that there are equivalent rates of victimisation and perpetration experienced by both men and women.

Emeritus Professor Michael Johnson is an American-based feminist sociologist and international expert on domestic violence. Since his original publication in 1995 he has published more than 40 articles on his typology and authored a book about it (Johnson, 2008). 
Central to Johnson's theory is that both feminist and family conflict researchers are correct in their findings and that they are simply measuring distinct types of IPV (Meier, 2015; Anderson, 2008). Johnson argued a failure to distinguish between these distinct types of violence has led to over 40 years of misleading and contradictory findings (Anderson, 2009). Johnson identified differences in the lens of the researchers, the methods used and the population studied, leading to studies measuring different forms of violence (Zweig et al., 2014).

Johnson's typology is not focused on the frequency or seriousness of the violence experienced in the relationship but is grounded by the presence, or not, of coercive control. As discussed in Chapter Two, coercive control is defined as a pattern of controlling behaviours that occurs over time and has elements that make it akin to an act of personal terrorism and stalking (Stark, 2007). Coercive control forms a pattern of behaviours throughout the relationship, where this psychological abuse means the perpetrator does not always have to resort to physical violence, as the control and threat of such violence is sufficient to keep the victim in a state of terror (Wangmann, 2011). Coercive controlling behaviours can include monitoring and surveillance of the victims, controlling their everyday lives, putting them down and diminishing their selfesteem and/or threatening to hurt them. Johnson argues the presence of coercive control is what distinguishes experiences of violence and helps explain the conflicting gendered results across studies (Meier, 2015). The nature of high coercive control makes for more terrifying and impactful violence.

In Johnson's typology, 'instrumental violence' - violence embedded in the need to assert or restore power and control - is labelled as intimate terrorism, while violence that is more expressive (emotionally driven and based around conflict) is labelled as Situational Couple Violence (Flood, 2019). As Stark (2006) states:

Michael Johnson $(1995,2000)$ reframes the debate about whether men and women are equally abusive by replacing the tenets underlying the controversy, that abuse should be equated with violence, that abuse can be assessed by a calculus of physical harms, that a single moral yardstick can be applied to any use of force by family members, and that, therefore, the issue of parity can be resolved by agreeing which family members use force, what kinds, and how often. Instead, he argues, because of the added element of "control," the 
"intimate terrorism" that affects women primarily involves a qualitatively different dynamic (and so different harms) than the "common couple" or "situational" violence in which surveys show there to be sexual parity. (Stark, 2006, p. 1019)

Johnson posits that within the different types of violence there is a range of trajectories in the establishment and maintenance of violence and different causes for each typological category (Anderson, 2009).

\section{JOHNSON'S DEVELOPMENT OF HIS TYPOLOGY}

Johnson first developed his theory that formed the basis of his typology of violence in the early 1990s. By the mid-90s he began to validate his theory through existing survey data on IPV.

Johnson first presented his early thinking on a typology of violence at a conference in Ho Chi Minh City in 1993. In this early conception of the typology he identified two types of violence - patriarchal terrorism and ordinary couple violence (Johnson, 2017). In 1995 Johnson published his first paper on his theory for the typology, although he had changed his terminology from ordinary couple violence to common couple violence (and subsequently to situational couple violence) after strong reaction to the term 'ordinary' as it appeared to minimise and excuse the violent behaviour it represented (Johnson, 2017). As part of re-labelling these categories, Johnson also shifted the definitions to allow for situational couple violence to represent cases that include severe and escalating violence, and violence that is not always mutually occurring. Patriarchal terrorism was also reframed as intimate terrorism, to acknowledge that violence between intimate partners is not always motivated by patriarchal beliefs (especially with a number of women being classified in this category), and can involve low frequency and low severity of physical violence in the context of high control (Meier, 2015).

To test and validate his theory Johnson conducted secondary analysis on two existing datasets, one that included a clinical and community sample (The Pittsburgh Data; Frieze \& Browne, 1989) and the other, a nationally representative sample collected from men and women (The Violence Against Women Study; Tjaden \& Thoennes, 1999). Both these studies collected data from women in the USA and included 
questions on women's experiences of violence in intimate relationships and a measure of control.

Through his analysis Johnson extended his two-category typology to include four categories: (1) intimate terrorism; (2) violent resistance; (3) mutual violent control; and (4) situational couple violence. A more detailed description of the four categories follows:

(1) Intimate terrorism is what Johnson describes as a pattern of one partner's use of frequent and severe coercive controlling non-violent tactics in the presence of physical violence (Johnson, 2008). Coercive control is where someone uses threats or negative consequences to get someone to do something they do not want to do. The coercive nature of the control often coincides with intimidation and threats (Johnson 2008). This form of instrumental violence is most likely associated with the term 'domestic violence' or 'battering' (Wangmann, 2011).

Central to the concept of intimate terrorism is the intention for one partner to exert power and control over their intimate partner. Physical violence is a tool used to gain and maintain that control, but it is embedded in a pattern of coercive control where the perpetrator uses a range of other tactics to maintain power and control and to create terror and ensure victim compliance. A general pattern of control combined with violence instils fear in the victim, as the controlling tactics become an active threat of violence. A victim who is not able to anticipate when controlling tactics will boil over into physical violence becomes uncertain of their partner's actions, which develops into forms of mental and emotional torture (Johnson, 2009).

(2) Johnson's category of violent resistance is characterised as victims of intimate terrorists who employ violence to resist the controlling and violent tactics used against them. Johnson found that victims of intimate terrorism reported non-violent and violent actions in the absence of controlling behaviours. This category describes violence that is defensive or retaliatory and an attempt to stop or prevent future control and violence by their intimate partner (Hines \& Douglas, 2010).

(3) According to Johnson (2008), mutual violent control is a rare type of violence where both partners are violent and use high levels of controlling tactics towards each other. In this sense, both partners are labelled 'intimate terrorists' (O'Neal, Tellis, \& 
Spohn, 2014). This category of violence is not about mutual use of force but about mutual deployment of coercive controlling tactics in the intimate relationship (Wangmann, 2011). "This pattern is basically two intimate terrorists battling for control in a relationship and is very rare” (Hines \& Douglas, 2010, p. 39).

(4) Situational couple violence is where one or both partners use forms of violence against each other but in the absence of high levels of controlling tactics. This violence is not about a general pattern of coercive controlling behaviours but is violence that is 'expressive' - in that it is largely reactive to a certain situation where it is emotionally driven and in the context of conflict (Flood, 2019; Wangmann, 2011). This category of Johnson's typology represents violence that is more likely to be equally reported by both males and females. The abuse is often less severe and less frequent than violence in a coercive controlling context, although some instances of situational couple violence do involve serious violence and injury (Wangmann, 2011).

Johnson argues that situational couple violence is what family conflict theorists likely find in national samples and is more likely to show findings of gender symmetry. However, a number of studies still demonstrate that the impact of situational couple violence has disproportionate impacts on women, who report greater levels of fear and injury than their male partners (Wangmann, 2011).

\section{PitTs BuRgh DATA}

Johnson's first analysis to test his typology was published in 1999 and used data collected for the Pittsburgh study (Frieze \& Browne, 1989). Data were collected in the 1970s from women who were married or had previously been married. The sample included interviews with 137 women who had experienced violence and from a comparison group of 48 battered and 89 non-battered women (Frieze, 1983). All participants were asked questions about their own use of violence and control and about their male partners' violence and control. The control scale in this study consisted of seven measures covering threats, economic control, use of privilege, using children, isolation, emotional abuse, and sexual control and included questions such as Has your husband (Have you) ever gotten angry and threatened to use physical force with you (him)? Johnson (2009a) used cluster analysis to categorise participants who had experienced violence into groups based on the level of coercive control reported in their relationships. 
The Pittsburgh study data was based on women who reported their use of violence and/or who reported their husband's use of violence. It showed that situational couple violence was by far the most prevalent in the survey sample ( $86 \%$ of men and $86 \%$ of women), with a much smaller proportion perpetrating intimate terrorism ( $11 \%$ of men and $3 \%$ of women). In contrast, the incidence of situational couple violence ranged from $28 \%$ to $37 \%$ for men and women in the court and shelter samples. These findings support Johnson's hypothesis that situational couple violence is more likely to be found in a community-based sample compared to a clinical sample.

Johnson's analysis also showed that men were more likely to be classified as perpetrating intimate terrorism compared to women who were more likely to report violent resistance. For example, in the shelter sample intimate terrorism was most likely to be reported by men (at $66 \%$ ), while the mirror was that $61 \%$ of women reported violent resistance. Analysis of the incidence of different forms of IPV across samples shows the gendered nature of this violence more clearly, with $89 \%$ of those categorised as intimate terrorists being men and almost all the violent resistance (85\%) reported by women.

Meanwhile situational couple violence had an almost even split with 55\% of men and $45 \%$ of women, suggesting a more gender-symmetric pattern of abuse in this category.

The data do not leave much doubt that intimate terrorism and situational couple violence are not the same phenomenon. We need to make these distinctions. (Johnson, 2008, p. 23)

These results provided some surprises for Johnson. He found that while intimate terrorism was more prominent in the court sample for men (at $46 \%$ ), the incidence of situational couple violence was also relatively frequent at $37 \%$. Similarly, $41 \%$ of women in the court sample reported violent resistance but $31 \%$ also reported situational couple violence.

I had expected agency data to be heavily dominated by men's intimate terrorism and women's violent resistance, but there were considerably more cases of situational couple violence than I had thought there would be. Where did I go wrong? I had mistakenly assumed that only intimate terrorism would frighten women enough to send them to the courts for a Protection from Abuse 
Order, or to a shelter for support or temporary housing. I hadn't paid close enough attention to my own statements that even situational couple violence can be quite frequent and/or severe. And I hadn't taken seriously enough the evidence from general surveys that men's situational couple violence is much more likely than women's to produce injuries. (Johnson, 2008, p. 21)

The analysis on the Pittsburgh data using Johnson's categories of intimate terrorism and situation couple violence confirmed that there were different experiences of violence found in different samples of women and men. As a result, Johnson continued to test his typology using secondary analysis on existing data, this time the Violence Against Women Survey data (VAWS) (Tjaden \& Thoennes, 1999).

\section{National Violence Against Women Survey}

The VAWS (Tjaden \& Thoennes, 1999) is a nationally representative telephone survey that collected data on several types of violence against women in the USA. The survey was carried out between 1995 and 1996 (Johnson \& Leone, 2005). Data included 8,005 men and 8,000 women aged 18 years and over. However, Johnson limited his analysis to include just 4,967 women who were married when the survey was administered (Johnson, 2008).

The survey used scales from the CTS and a modified non-violent control scale taken from the equivalent Violence Against Women study in Canada. Johnson used the nonviolent control scale to operationalise his typology; he used a k-means cluster analysis to categorise respondents who reported that their male partner had been physically violent into intimate terrorism or situational couple violence clusters.

Further analysis of this data supported Johnson's theory that relationships characterised by intimate terrorism produced far more serious behaviours, including greater frequency and severity of violence, when compared to situational couple violence (Johnson \& Leone, 2005). Women who experienced intimate terrorism were more likely to report days off work, to be injured and were more likely to display symptoms of post-traumatic stress disorder (PTSD) compared to women who experienced situational couple violence. 


\section{TESTING THE TYPOLOGY}

Johnson's typology has been "met with remarkably rapid and widespread adoption" (Wangmann, 2011, p. 2) and has attracted significant attention with the term 'intimate terrorism' being used in 204 articles between 2000 and 2013, and more since (Straus \& Gozjolko, 2016). As a result, a number of researchers have conducted studies to test Johnson's typology using similar methods to his, or by extending his analysis to include different relationship types (e.g., not just married women), male and female respondents, and by applying other statistical methods to create the typology categories.

Graham-Kevan and Archer (2003) replicated Johnson's typology in a sample of British men and women. The sample was small but included females recruited from a women's shelter, a mixed-sex student sample, four men who were attending a domestic violence treatment programme and 97 male prison inmates. Each participant reported both their own and their partner's use of violence in a relationship. Graham-Kevan and Archer (2003) used a modified version of the Conflict Tactics Scale and also the Controlling Behaviours Scale (Pence \& Paymar, 1993) to determine which category of Johnson's typology participants should be assigned to. Consistent with Johnson's findings, they found that situational couple violence ( $62 \%$ males; $77 \%$ females) was more commonly reported compared to intimate terrorism (33\% males; $6 \%$ females) in the community sample.

Also consistent with Johnson, Graham-Kevan and Archer (2003) reported that those in intimate terrorist relationships were typically more likely to report frequent, escalating violence that was "one way" while situational couple violence relationships were more likely to be characterised by less frequent and non-escalating violence. They also found that men were more likely to report intimate terrorism and women were more likely to report violent resistance - however when the shelter sample was omitted from the sample there was no difference in gender. Likewise, situational couple violence was gender symmetric when both partners were violent (mutual violence), however, when looking at cases where there was only one partner that was violent within the situational couple violence category, surprisingly women were more likely to be the sole perpetrator of the violence. 
Rosen, Stith, Few, Daly, and Tritt (2005) applied Johnson's typology to their analysis of qualitative interviews. They sought to understand the characteristics of those classified into Johnson's categories and to examine whether there were any couples that did not fit into his categories. They conducted interviews with 15 couples aged 18 years and over. Rosen et al. (2005) confirmed that the couples in their study broadly fit into three of Johnson categories although the intimate terrorist category did not apply to any of their cases. They hypothesised that this was likely because victims experiencing intimate terrorism did not feeling safe participating in such research and therefore this category was not present in the study. However, they concluded that their findings supported Johnson's typology and the argument that it is necessary to distinguish between different experiences of IPV and the context within which this violence occurs.

Other studies that have sought to develop typologies of IPV have identified categories that overlap with Johnson's categories. Pence and Dasgupta (2006) developed five categories ${ }^{1}$ using 15 years' worth of interviews from men and women in Duluth, Minnesota, who were arrested for domestic abuse as well as a review of police and court documents that represented a more clinical-like representation of those experiencing IPV. While not directly testing Johnson's typology, Pence and Dasgupta (2006) identified five categories, three of which corresponded with Johnson's - a category called 'battering' that aligns with intimate terrorism, a category similar to violent resistance called 'resistive/reactive violence' and a category called 'situational violence' that mirrors Johnson's situational couple violence category. They concluded from their analysis that, while there is a need to continue to acknowledge the role of power and control in men's use of violence against women, there is a need to understand the "dynamics, nuances and components of IPV with the idea that this critical comprehension is vital in creating intervention and prevention models that befit the behaviour" (Pence \& Dasgupta, 2006, p. 4). However, until there are reliable, effective tools in making such distinctions, they conclude the need to "err on the side of caution" when intervening (p. 17).

\footnotetext{
${ }^{1}$ The five categories are battering, resistive/reactive violence, situational violence, pathological violence, and anti-social violence.
} 
Similarly, Hines and Douglas (2010) did not directly test Johnson's typology but did apply his theory from a conceptual perspective when understanding data from a study of a sample of 302 men who were seeking help as victims of IPV, and a comparison group of men in the community. Hines and Douglas (2010) observed mostly reciprocal violence in the community sample, which was similar to Johnson's situational couple violence category. They also reported that the men in the help-seeking group, which they posit were comparable to samples of battered women, described their partners enacting violence that resembled Johnson's intimate terrorism category. Their female partners used a range of violent acts and controlling behaviours at a significantly higher rate than the male help-seekers. These men also reported experiencing significantly higher numbers of violent acts over the previous 12 months and also significantly higher rates of injury compared to the community sample of men. Hines \& Douglas (2010) conclude that:

taken together, there is strong evidence that the female partners of men in the help-seeking sample conform to Johnson's (1995) conceptualisation of intimate terrorism. This is not violence that can be viewed as trivial, humorous or merely annoying, as some have suggested. (p 52)

Although these previous studies provide some support for Johnson's typology, other studies have found conflicting results that suggest that his typology may not provide significant value above and beyond already existing measures in regard to predicting violence and acting in a responsive and appropriate manner (Graham-Kevan \& Archer, 2008; Frye, Manganello, Campbell, Walton-Moss, \& Wilt, 2006; Anderson, 2008; Gulliver \& Fanslow, 2015).

A study by Graham-Kevan and Archer (2008) of 113 male and female students, 43 women from a shelter and 108 male prisoners, tested the association between spousal violence and controlling behaviours. The sample was effectively doubled, as responses from respondents' partners were also included, creating an artificially constructed sample of 486 unique cases for analysis. When looking at levels of controlling behaviour across the different relationship categories, they found that controlling behaviour was a significant predictor of physical aggression regardless of how the violence was categorised. 
Frye et al. (2006) also conclude that the presence or absence of control does not clearly differentiate between different types of IPV. Frye and colleagues directly assessed Johnson's typology using a sample of 331 physically assaulted women across 11 North American cities. They found that control was present across $69 \%$ of women who reported being physically assaulted, suggesting that intimate terrorism and situational couple violence may not be as distinctly different as Johnson hypothesised when using control as the differentiating variable. In fact, when escalation and injury were factored into the analysis a more fine-grained distinction between experiences of violence was found. Frye and colleagues suggest that there are not two distinct typologies but rather that control, injury and escalation of violence are factors that characterise different experiences of violence that may change or 'evolve' over time. Surprisingly Frye and colleagues also found that situational couple violence was reported at a low rate in the population sample, which raises questions about Johnson's hypothesis that violence within a community sample is more likely to represent situational couple violence compared to clinical and shelter samples.

Anderson (2008) examined Johnson's typology when carrying out analysis on a sample of 4,575 married women who had taken part in the NVAWS in the USA. She looked to see whether Johnson's typology, when compared to other measures of violence, explained the variation in a range of negative outcomes such as the number and type of violent acts used. She reported mixed support for Johnson's typology, as it added little value when compared to a more simplistic measure of the number and types of violent acts experienced by the respondents. Anderson also found a group of women who reported harmful levels of relationship control in the absence of physical violence, and she called out the limitations of Johnson's typology in not including harmful non-physical relationship violence. This is an important finding given what we know about high control reducing the need for physical violence.

Straus and Gozjolko (2014) categorised an international dating violence study sample of 13,877 university students into Johnson's categories and found that $26 \%$ of women and $20 \%$ of men reported high use of control, therefore qualifying them as intimate terrorists. Straus and Gozjolko stated that the relatively high percentage of young adults classified as intimate terrorists is inconsistent with the intention of creating a category that reflects less frequent but more damaging cases as reported by battered 
women or seen in the criminal justice system. Straus and Gozjolko (2014) surmised that Johnson's inadequate methods mean the intimate terrorist label is misleading and that this type of violence needs to be a reflection of "frequent or severe assaults, injury, fear or subjugation in order to warrant classification as intimate terrorism" ( $\mathrm{p}$. 62). Likewise, Jasinski, Blumenstein and Morgan (2014) applied Johnson's procedure to a sample of married men and women from the NVAWS. Of the women who reported being a victim of IPV, $36 \%$ could be classified as a victim of intimate terrorism, while $35 \%$ of male victims of IPV could be so classified. Further analysis of the gender differences of those classified as intimate terrorist also failed to show any differences between frequency and severity of the violence. They too concluded that control alone was not the most adequate way to classify differences in violence experiences when the control is not measured in context. As Jasinski and colleagues (2014, p. 85) explain "the crucial element to consider is how much power does each partner bring to the relationship to turn those acts of annoyances into actual coercion? In other words, which partner is actually able to maintain and enforce her or his control."

More recently, Gulliver \& Fanslow (2015) tested Johnson's typology using latent class analysis to identify the types of relationships experienced in a population-base sample of New Zealand women. They found that they were unable to replicate Johnson's categories and found that there was not a mutually exclusive relationship between his categories when control was used as the distinguishing variable. Instead they posit that their findings support an approach that focuses on a continuum of violence. They also concluded that their results support the need to further investigate typological approaches and their utility in understanding the complexity of violence in intimate relationships, particularly given that:

violent relationships are neither static nor homogeneous and are fuelled by issues of control and interdependence, neither of which have yet been adequately understood or measured. (Gulliver \& Fanslow, 2015, p. 41)

These studies showed mixed support when replicating Johnson's typology. 


\section{GENDER DIFFERENCES}

One of the main reasons for Johnson's typology was to develop a way to reconcile differences found between family conflict and feminist-based theorists on the role of gender. Johnson posits that by classifying respondents into his typology, symmetrical violence will be identified across situational couple violence where men and women will report similar levels of perpetration, while men will predominantly be the perpetrators of intimate terrorism. A small number of studies have specifically investigated the role of gender across Johnson's categories, particularly focusing on the gendered nature of intimate terrorism.

Bogaerts, van der Veen, and van der Knaap (2011) analysed data from 320 participants in the Netherlands, which was a subset from an online nationwide study carried out in 2008 on domestic violence. The 320 participants in this study were included as they reported perpetrating violence against a current or ex-partner, and included 119 male (40\%) and 201 female (60\%) respondents. Consistent with Johnson's hypothesis, Bogaerts et al. found that men were more likely than women to be categorised as intimate terrorists, $21 \%$ and $14 \%$ respectively.

In contrast, Graham-Kevan and Archer (2004) reported results that contradicted Johnson's hypothesis, in that more women than men were categorised as intimate terrorists. Graham-Kevan and Archer (2004) carried out a study with a sample of 1,339 students and staff from a UK university. Results showed that $9 \%$ of men were categorised as intimate terrorists compared to $13 \%$ of women. Likewise, in their 2014 publication Bates, Graham-Kevan, and Archer found that there were no differences in the use of controlling behaviour between male and female participants, and when adopting Johnson's typology women were, in fact, more likely to be categorised as intimate terrorists. Laroche (2005) categorised participants from the 1999 Canadian General Social Survey of victimisation and spousal violence, and also found that more women were categorised as intimate terrorists than men, $26 \%$ and 19\% respectively.

Similar results were reported by Hines and Douglas (2010) who analysed a sample of 520 men from a community cohort. They categorised participants' responses about their partner into high and low control, thus creating categories in line with Johnson's typology. They reported that women were nearly twice as likely as men to be categorised as high control, $20 \%$ and $12 \%$ respectively. These results suggest that 
while some studies demonstrate that men are more likely to be categorised as intimate terrorists, particularly when they involve clinical samples, this is not always the case. Analysis of community and general population samples suggest that both men and women can be classified as intimate terrorists.

While this section provides some support for Johnson's typology there are also a number of studies that reported results inconsistent with Johnson's hypothesis and conclusions. These inconsistencies highlight potential issues with his typology, and more generally in the creation and utility of typological research.

\section{LIMITATIONS TO JOHNSON'S TYPOLOGY}

Limitations with Johnson's typology have been documented by a number of scholars such as Meier (2015) and Wangmann (2011) and it is important to address these before progressing with a discussion on the utility and application of his typology. One potential limitation to Johnson's protocol is his concept of coercive control. Within Johnson's typology he classifies anyone with three or more control tactics as high coercive control, with little explanation for this cut-off and its applicability to other samples. It also raises the question as to whether confirming the use of one of the control tactics qualifies the respondent to be classified as coercively controlling without understanding more about the gravity of that control tactic and the context within which the control is enacted (Meier, 2015).

Another area identified through the qualitative inquiry where Johnson's typology appears weak, was the general narrative underpinned by attitudes and beliefs that reinforced traditional gendered norms and the use of violence. The use of coercive control is an important element in Johnson's typology as it is essentially an exaggerated view of gendered stereotypes where male dominance is normalised and females enact a submissive role (Swan, Gambone, Caldwell, Sullivan, \& Snow, 2008), which creates the foundation for use of physical violence in an intimate relationship (Pence \& Paymar, 1993). However, this raises the question of whether coercive control is the primary variable to distinguish between different patterns of violence, and is there just one variable to do this? The interview findings bring into question the role of the strong cultural and social contexts that drive attitudes and behaviours supportive of 
gendered norms and attitudes supportive of violence, and how they may be integrated into an approach to classify types of violence (Nybergh, Enander, \& Krantz, 2016).

Linked to this is the fact that Johnson's typology is based on individuals who use coercive control in the context of physical violence, therefore there is a possibility that there is missing information around relationships where other violent and controlling tactics are used to enforce control, outside of the presence of physical violence. There is strong evidence that control in the absence of physical violence can have significantly negative outcomes, with the use of psychological aggression being reported as more harmful for both male and female victims compared to physical violence (Anderson, 2008)

\section{A central limitation of the use of the intimate terrorist/situational couple violence typology is that it does not consider the effects of high relationship control apart from the context of physical violence. Indeed, women who do not experience physical violence are not identified as victims by the intimate terrorist/situational couple violence typology even if they experience extremely high levels of the controlling behaviours described as terrorism by Johnson. (Anderson, 2008, p. 1166)}

More broadly there are suggestions that coercive control has been a neglected area in the research literature with the emphasis on more visible physically violent relationships (Stark, 2007). Johnson's focus on control in the context of physical violence raises the question of how to treat high control in the absence of physical violence and what we are missing when we omit relationships where there is control in the context of other violent and aggressive behaviours, including psychological or sexual violence. A way to resolve this limitation is to extend Johnson's typology to include relationships where control is present in the absence of physical violence; this is discussed in Chapter Eleven in the section on Future Research Areas and Possibilities.

Another possible limitation that could impact the results of this study's ability to more fully support Johnson's typology, is whether there are different types of violence or whether abuse is on a continuum. Dating violence is more often representative of early stage of relationship formation and therefore violence looks less severe than that in 
relationships that are more well established. Meier (2015) described the possible scenario where some studies could be capturing information early in a relationship where there is violence but little control. This same relationship could be re-classified as it progresses when the perpetrator has increased their power and control and the effects of this long-term control and violence have instilled fear and caused severe negative impacts for the victim. If the typology is merely picking up the same couples at different points in their violent relationships then the typology itself is far less salient because future harm and impacts of violence cannot be distinguished, meaning the typology descriptions do not meaningfully and helpfully apply to violent experiences (Meier, 2015).

Given these limitations I would be cautious about the wholesale application of Johnson's typology in practical terms as there are risks in applying such a typology. In a review of Johnson's typology, Australian academic Jane Wangmann (2011) discussed the implications of misclassification that may result in the system responding in a way that could increase the risk of harm to the victim. Wangmann (2011) quotes from Pence and Dasgupta (2006) who articulate the risk of wrongly categorising a victim/violent couple:

Practitioners and advocates may only get one chance to successfully intervene in a victim's bid to end violence and hold the batterer accountable for his behaviour. Misjudging battering for other kinds of violence ... and intervening incorrectly might make the difference between life and death for a victim. Thus, until we can create highly sensitive, valid, and reliable diagnostic tools and techniques to identify batterers, we can hardly risk any errors in our assessments ... before such a time arrives, we would rather err on the side of caution. (p. 17)

As this quote highlights, the risk of misclassification can mean that our attempts to intervene, particularly in intimate terrorist-style relationships, could put the victim in harm's way or cause more psychological distress if support is not delivered in an appropriate way. Johnson himself is cautious about using his typology as a classification tool for intervention and aware of the implications of making incorrect classification. He states that "it is probably wise to assume that all violence is intimate terrorism until proven otherwise". (Johnson 2008, p. 82). 
Alongside the risk of misclassification is the risk of reinforcing minimising attitudes towards violence in intimate relationships. For example, there could be a perception that situational couple violence is common and perceived as less severe by the justice system thus reinforcing myths that it is not that serious, it was just momentary loss of control and that men and women are equally as violent (Wangmann, 2011). Meier (2015, p. 23) states that the label situational couple violence "intrinsically minimizes the severity of any violence and makes it sound mutual". Likewise, Pence and Dasgupta (2006) raise concerns about the labelling of this category, saying it could be used to inappropriately minimise and excuse the behaviour of violent individuals who present a serious risk to their partners. As such some researchers suggest there is a need to be cautious when attempting to apply theoretical typologies into practice and decision-making:

To ensure effective and safe intervention in families where IPV is being perpetrated, this training needs to include a focus on recognizing patterns of behavior rather than focusing on incident-based physical violence... At present, typologies are too under-developed to be used to guide clinical decisionmaking. (Gulliver \& Fanslow, 2015, p. 41)

While there are limitations with Johnson's approach, the body of research presented above does highlight the importance of viewing IPV as a complex phenomenon, and creates the opportunity to deviate from a one-size-fits-all approach to prevention and intervention (Flood, 2019). Johnson's typology has shone a light on the study of IPV in that it can no longer be usefully viewed as a unitary phenomenon (Kelly \& Johnson, 2008; Wangmann, 2011). Throughout this chapter, the evidence presented across a number of studies supports elements of Johnson's typology. Given that IPV research is founded on contentions between the role of gender and measurement and sampling issues it seems that the competing findings presented here are not surprising. As there is some empirical support for Johnson's typology or modification and extensions of his typology, it seems appropriate for this thesis to acknowledge its limitations while using it to build a wider understanding of the different experiences of violence, and how this can be applied to violence experienced in dating relationships in the New Zealand context. This study is also looking further into Johnson's typology through qualitative enquiry which will provide further support, or otherwise, on its application. 


\section{CHAPTER SUMMARY}

In this chapter the background to the development of Johnson's typology was outlined along with research supporting his work, while the limitations and concerns held by others researching IPV and dating violence were canvassed.

Despite criticism and apparent contradictions, Johnson's typology has support from a number of studies that have sought to replicate his typology, and as such it is widely accepted as a framework to represent the complexity of experiences of violence and the differing roles of gender. This chapter highlights the potential value in distinguishing between types of violence as differentiation can support a focus on designing and developing policy, services and prevention to address the different needs of those in a range of violent relationships (Wangmann, 2011). It is therefore considered acceptable to use Johnson's typology as a basis for this thesis.

The next chapter will describe the methodology used to measure the level of violence experienced by young adults in dating relationships in New Zealand and to explore the validity of Johnson's typology by replicating his methodology through a quantitative online survey. From this I aim to explore the utility of the typology in understanding the complexity of dating violence experiences with a view to assessing its potential to act as a valid framework for understanding the different experiences of violence in dating relationships. 


\title{
CHAPTER FOUR - Method
}

\author{
Mixed methods research can benefit any area of study ... and is particularly \\ appropriate for studying physical and sexual violence ... a complex, \\ multifaceted phenomenon, occurring within a social context that is influenced \\ by gender norms, interpersonal relationships, and sexual scripts. (Testa, \\ Livingston, \& VanZile-Tamsen, 2011, p. 237)
}

In this chapter I detail my approach in answering the research questions posed in this study. I begin with one of the foundational elements I used to refine my research focus and approach, which was through interviews with a number of key informants. I then set out the design and implementation of the quantitative online survey. Finally, I discuss the approach taken for the qualitative in-depth interviews

My motivation for this thesis was to understand more about the role of gender within intimate partner relationships, particularly in the context of young adult dating relationships in New Zealand. As detailed in Chapters Two and Three, following on from research into the experiences of female victims of IPV at the hands of their male partners, a growing body of work began to evidence that women were as violent as men, and that IPV was often bi-directional, sparking claims that there is gender symmetry in the perpetration of violence in intimate relationships (Douglas \& Straus, 2006). The hotly contested role of gender in the perpetration of IPV continues, and as a result a number of theories and typologies have been developed. The focus of Chapter Three was on one of those typologies, developed by Johnson (2008) to reconcile the differences in the findings on the role of gender. For this reason I chose to explore the utility of Johnson's (2008) typology of violence within the current New Zealand context of dating relationships.

\section{KEY INFORMANTS}

With a focus on replicating and testing Johnson's typology I was privileged through my job to work with a number of experts in the field of family violence in New Zealand who agreed to be key informants for this study. These key informants were critical in supporting me to shape and narrow my research focus. With their assistance I was able to test and discuss my ideas, to work through gaps in my logic and to refine 
and focus my research. Specifically, I talked with my key informants about my proposed research questions and methodology; to gather best practice in research on sensitive issues, advice, and experience from researchers in this area and/or who work with the study population (victims and perpetrators); to gather information on key theories and debates; to understand how the research could inform practice and policy; and to discuss recruitment of and access to young adults to take part in this study.

Appendix 1 lists the key informants that I talked with and the role and expertise that they brought to the conversations. They represented a range of views, occupations and organisations, including working in government and non-government sectors; in crisis support; across the prevention spectrum; with Māori and Pacific people; and in social research.

To engage the identified key informants, I emailed them an outline of my proposed study including the aim of the research, the research questions, the literature that was drawn on, and the proposed method and approach. The email also included the topic areas to be discussed with them (while allowing space for new thoughts, ideas and theories to emerge). I arranged times to speak with the key informants who were available to participate. Where possible I arranged to speak with them in person, although phone interviews were needed for those who were not based in Wellington. The discussions lasted approximately an hour and were recorded with their consent.

I used the learning and recommendations from my key informants to adapt the research questions and direction of the research. For example, the original research question was to look at pathways and the nature and consequences of dating violence but through the interviews the research aim and research questions became more focused on testing the applicability of Johnson's (2008) typology of violence and on capturing young adults' narratives on dating and experiences of dating violence. These areas were identified as critical as little is known about the adaptability of the typology to other relationships and ages (e.g., young adults in dating relationships) and the ways different types of violence are experienced by young adults. This could have significant implications for how 'types' of dating violence are addressed by the family violence sector (e.g., the type and intensity of interventions based on different motivations and outcomes). Some of the other main themes or lessons that came out of the key informant discussions included the need to focus on the gendered nature of 
violence; a focus on power and conflict dynamics; thinking about couple conflict versus violence; and what is included in the definition of violence.

\section{RESEARCH APPROACH}

From here I developed my final research questions, which were presented at the end of Chapter One. My resulting focus was to measure the level of violence experienced by young adults in dating relationships in New Zealand and to explore the validity of a published typology of violence by replicating the methodology used by Johnson (2008) through a quantitative online survey. From this I aimed to use qualitative interviews to explore the utility of his typology in understanding the complexity of dating violence experiences with a view to assessing its potential to act as a valid framework for understanding the different experiences of violence in dating relationships.

To address these questions a mixed methods study was designed, where measures were collected using multiple methodologies (Barnett, Miller-Perrin, \& Perrin, 2005). The strength of using a mixed method approach in this thesis was that valuable quantitative survey data was enhanced through the voices of young adults collected in the qualitative interviews. The intensive process of conducting interviews provided a depth of insight into the experience of dating and violence that could not feasibly be collected through survey methodologies yet was a pivotal complement to support analysis and understanding of the survey data.

This design allowed for synthesised multi-method evidence of the experiences of dating violence, young adults classification into Johnson's typology of violence and the narrative of young adults around their lived experiences of violent dating relationships in young adulthood. This mixed method approach, coupled with key informant data and current national and international literature, allowed for triangulation of sources. Triangulation is the use of multiple methods of data collection to study the same phenomenon and to more fully explore research questions from a number of data perspectives. Triangulation of data provides a rich and diverse view and can present a wider range of perspectives across research studies (Tashakkori \& Teddlie, 2003). 


\section{FOCUSING MY SAMPLE}

As detailed in Chapter One, I focused on young adults aged 18 to 25 years old, an age when young people often start to establish more long-term relationships and/or relationships where they take on roles and responsibilities close to those of married couples. They are also likely to become more independent as they leave home, enter into training or education, and find employment. The sample in the online survey and in the qualitative interviews was limited to never married 18-25 year olds. This allowed for the inclusion of a range of degrees of relationship seriousness.

To extend this study sample beyond students, I specifically recruited participants not only through universities and polytechnics but also through crisis support agencies. I employed this recruitment frame to ensure experiences of violence across the continuum of seriousness were included, while capturing the views of those immersed in university culture where the social context of drinking and casual sexual encounters was most likely to be found.

While not deliberately excluded, young adults in same-sex relationships were not a specific focus of this research. The findings are largely limited to a heterosexual perspective, although a number of young people did identify that they were in same sex relationships $(\mathrm{n}=28,7.5 \%)$, with one interview participant referring to their samesex relationship in their experience of dating violence. The heterosexual lens does limit the scope of this research, particularly as members of marginalised groups are disproportionately impacted by dating violence and other forms of intimate violence (Beres, 2017; Dank, Lachman, Zweig, \& Yahner, 2014; Freedner, Freed, Yang, \& Austin, 2002). This research was primarily focused on a heterosexual perspective of dating violence but there is opportunity and need for this research to be extended to same-sex relationships as discussed in Chapter Eleven.

Deliberate attempts were made to ensure the representation of young Māori in this study (see later in this chapter on recruitment techniques for the online survey including targeting specific university courses that had higher representation of Māori and Pacific students). Māori are the indigenous people of New Zealand and are disproportionally impacted as a result of structural inequalities, colonisation, poverty and from living in a Westernised society that does not fully recognise or value a Te Ā̄ 
Māori perspective (the Māori world) (Beres, 2017). This includes being overrepresented in statistics on victimisation and perpetration of IPV and other family violence statistics (Dobbs \& Eruera, 2014). As reported in Chapter Five, in total 35 survey respondents identified as Māori (9.4\%) and 23 reported that their current or expartner was Māori (6.1\%). In the interviews one male participant identified himself as being Māori and one female participant reported their partner was Māori. This provides an overall under-representation for those identifying as Māori, which means that there are limitations from a diverse ethnic perspective. The impact of the sample and need for culturally-specific research, frameworks and solutions to prevent and support young Māori engaging in dating relationships are discussed in detail in Chapter Eleven (Eruera \& Dobbs, 2010; Beres, 2017).

\section{QUANTITATIVE ONLINE SURVEY}

With my research focus and approach finalised, I began to develop a quantitative online survey to address the first research aims. The survey was developed to measure the level of violence experienced by young adults in dating relationships in New Zealand and to explore the validity of a typology of violence by replicating Johnson's methodology (M. Johnson, Personal Communications, 12 November 2014). I utilised a range of existing measures to assess the level of dating violence experienced and to replicate Johnson's (2008) typology of violence.

Two main populations were targeted for the online survey: the student population and a crisis support agency sample. The purpose of collecting data from these two samples was to ensure that a range of experiences from minor to severe violence were captured, and subsequently to allow for robust testing of Johnson's (2008) typology. Accessing clients from crisis support agencies provided a targeted sample where violence had occurred.

The participants in the study self-selected into the survey producing a convenience sample. It was not possible to select a random sample of young adults from within the student and non-student populations within the frame of this study. While this convenience sample limits the generalisability of the results to the wider New Zealand young adult population, it does give an insight into the characteristics of university and polytechnic students (range of ages, ethnic groups, education levels) and crisis support 
agency clients (range of experiences in intimate relationships). The lack of a random sample for the online survey is deemed as acceptable in this study as it is not a prevalence study but aims to provide an understanding of the types of dating violence experienced by young adults in New Zealand and to inform the qualitative study.

\section{MeAsures}

Through my review of the literature I identified key variables linked to experiences of violence and key scales and measures used to establish the amount of violence experienced, levels of control, and the impact of the violence. Primarily, I included the measures Johnson used to create his typological categories in order to be consistent in the development of the typology. These measures included the CTS2 (Straus, Hamby, Boney-McCoy, \& Sugarman, 1996) and the Coercive Control Scale (Johnson, Leone, $\& \mathrm{Xu}, 2008)$, both of which are described below. A range of demographic information was also collected.

\section{The Revised Conflict Tactics Scale (CTS2)}

I used the Modified Revised Conflict Tactics Scale (CTS2; Straus et al., 1996) to establish the occurrence of physical assault, psychological aggression, sexual coercion and injury experienced by this sample of young adults. The original Conflict Tactics Scale (CTS, Straus, 1979) was revised as a result of a range of criticisms. These included the original CTS's inability to provide context to the types of violence used including severity (Barnett et al., 2005). Fuller discussion on the CTS2 is covered in Chapter Two. While there are limitations to the CTS2, it is still widely used to measure experiences of violence in relationships and was used by Johnson (2008) to develop his typology, which is being replicated in this study. The following subscales from the CTS2 were included - physical assault, psychological aggression and sexual coercion.

Examples of items in the CTS2 include "how often have you done the following to a current or ex-partner: threw something at my partner that could hurt; beat up my partner; insulted or swore at my partner; insisted on sex when my partner did not want to; placed unsolicited phone calls to them; vandalised their property or destroyed something they loved". All of these questions ask "how often" the respondent's current or ex-partner has done this to them and how often they have done the same to a current or ex-partner (for example see: Straus et al., 1996). 
The injury scale from the CTS2 (Straus et al., 1996) was also used in this study. This scale was developed from data collected through the National Family Violence survey, conducted in 1985. The scale asks how often types of injury occur. The scale includes six items that described types of violence that the participant could report experiencing. Examples of items used to measure injury include "I had a sprain, bruise, or small cut because of a fight with my partner", "I went to a doctor because of a fight with my partner" and "I had a broken bone from a fight with my partner".

\section{Coercive Control Scale}

The final scale that was included in this study was the Coercive Control Scale that Johnson used in his studies (Johnson et al., 2008). Johnson (2008) describes control as a continuum where everyone controls his or her partner to some degree in a relationship. When the continuum moves to a place where 'coercion' is used, is where control can have a significantly negative impact. Coercive control is where someone uses threats or negative consequences to get someone to do something they do not want to do. The coercive nature of the control often coincides with intimidation and threats (Johnson, 2008). Examples of items used to measure coercive control include "how often is your ex- or current partner jealous or possessive" and "how often does your ex- or current partner try to provoke arguments".

The survey also included questions to establish the demographic characteristics of those who participated, including their age and their partner's age; their ethnicity and their partner's ethnicity; their gender and their partner's gender; relationship status (dating or engaged); length of the relationship; if they were New Zealand-born, if not then the length of time living in New Zealand; where the participant currently lives; living status (e.g., flatting, at home, with a partner, on their own, etc); current employment status; their income; total number of partners; and how they found out about the survey.

\section{DEVELOPING THE ONLINE SURVEY}

Through the scoping stage I reviewed methodologies from other studies and identified an online survey as the most reasonable way to collect information on experiences of dating violence. An online survey allows anonymity for participants, which can increase disclosure of sensitive issues such as relationship violence; participants can 
also take part in the survey when they are ready. Additional benefits of an online survey are that the software can manage skips and 'logics' in the survey so participants do not need to manage what answers they are required to respond to; the software collects data in a form for analysis which limits potential data entry errors; and it outputs datasets in ready-to-use formats for most statistical software packages.

These days computer access is less of a limitation for participation in an online survey. All students have access to computers through their university or polytechnic, making access and participation optimal. For the crisis support agency sample, computer and Internet access may not be as readily available or easy to access, and for this reason I gave participants the option to complete the survey in paper form. This option was not taken up by any participants.

The survey was developed by combining the identified measures and replicating similar surveys in the literature, this included the best sequencing of questions, items and scales; the format and length of the survey. Once the survey had been drafted, I carried out cognitive testing face-to-face with young adults to test the understanding and meaning of the questions. In other words, were these young adults answering the questions as intended or were they interpreting what the questions were asking in a different way? This process also allowed for testing of the terms in the survey. Most of the scales have been developed overseas and few have been used in the New Zealand context, so it was important to make sure the (mostly American) language was 'kiwi' enough and the terms were consistently understood in the New Zealand context. The most significant term tested was 'dating'. As noted earlier, this is a term used largely in the American literature, so it was important to test if this term had the same meaning for young New Zealanders, even if it is a term that is not commonly used to label such relationships. During cognitive testing the term 'dating' was explored for its utility as a term to explain relationships that ranged from hook-ups that last more than just one encounter, through to relationships or pairings where there was some level of exclusivity, from a less serious short-term relationship to a more serious 'pre-marital' relationship. These range of relationship types were understood by these young adults to include forms of 'dating' practices and therefore the term 'dating' was deemed as appropriate to use throughout the survey. 
Once the cognitive testing was complete, the order/sequencing of the survey was finalised. The sequencing was as follows: a consent page; screening questions (age, ever married, length of relationship); Coercive Control Scale; CTS2; demographics; and permission to contact for the qualitative in-depth interviews.

The survey was then entered into the online survey tool, Qualtrics. Qualtrics is an easyto-use web service that provides an intuitive interface with point and click functionality; online reporting and data export functionality; ability to publish reports online that update as responses are received; and one click data export to Excel, CVS and other formats. Qualtrics can be accessed from any computer with an Internet connection. At this point I piloted the survey to test the length, ease of finish and that skips and logic in the survey were operating properly.

I then developed a study website to provide an easy link for participants into the survey. The website was developed using iWeb (an Apple-based product) and included information about the survey such as its purpose, the consent process and ethical approval. Having this information on the web page meant that the online survey tool was not cluttered with information and meant that once a participant had opted into the survey by giving consent they would be directed straight to the start of the survey. Each page in the website had a link to the online survey so participants could start whenever they were ready. (See Appendix 9for screenshots from the website).

\section{RECRUITMENT OF PARTICIPANTS}

To recruit participants into the study, I developed posters that I placed on notice boards around university and polytechnic campuses and gave to crisis support agencies to circulate. A PowerPoint slide was also developed and emailed to heads of schools to be presented in lectures and classes. Heads of schools were identified through classes that had large numbers of students, including Māori and Pacific students to maximise possible respondents. These classes included undergrad 100-300 level classes such as Psychology, Mathematics, Criminology, and Business.

The uptake of participants through crisis support agencies was less than I had anticipated. I was only able to recruit participants through the posters placed in agency waiting rooms (the implications of this will be discussed in Chapter Eleven). To increase participation of those who had accessed crisis support agencies and/or who 
had experienced more severe violence, I asked key informants to identify individuals who were sent the link to the survey if they were happy to participate in the study. A snow-balling technique was also used, where participants passed on survey information to other contacts who then took part in the survey.

As a result of this adapted recruitment process, the exact number of survey participants who would be classified as the 'crisis support sample' could not be calculated due to the survey question that asked how participants found out about the survey. Respondents could either reply "through a poster on campus", "through a poster at an agency" or "through word of mouth". The number of participants recruited through "word of mouth" will include those who were recruited through key informants as well as participants who found out about the survey from other avenues (e.g., through Facebook).

The online survey ran for 18 months (from November 2011 to November 2012). During this time in-depth interviews were carried out with participants who consented to being contacted for this purpose. It was important to contact willing participants immediately as waiting until the end of the survey data collection phase might mean that participants were no longer willing or able to take part, that contact details were no longer valid or that the circumstances that they reported in the survey were no longer current.

\section{QUANTITATIVE DATA ANALYSIS}

The primary aim of the quantitative data analysis was to measure the level of violence experienced in dating relationships in New Zealand and to validate and replicate Johnson's (2008) typology.

As detailed above, the Coercive Control Scale was used to replicate Johnson's typology (Johnson et al., 2008); the critical component, alongside the CTS2 responses, used to develop Johnson's typology. It measures respondents' own controlling behaviour and the controlling behaviour of their dating partner and consists of nine items.

In applying Johnson's analysis protocol to develop his typology, I first categorised participants and their partners into no, low or high control groups. Johnson's 
recommended cut-off was used to determine high and low control, whereby those who reported experiencing no acts were classified as experiencing no coercive control, those who reported fewer than five acts were considered low control and those who reported experiencing five or more coercive controlling acts were classified as high control (Personal Communications, 12 November 2014).

Following the classification of respondents and their partners into low, high or no coercive control categories, respondents were coded into Johnson's typology following his procedure (Personal Communications, 12 November 2014). First, respondents and their partners were split by those who have perpetrated physical assault and then by the level of coercive control they enacted. From this, they were classified into one of three categories: situational couple violence; intimate terrorism; or, non-violent.

The benefit of collecting information on both partners is the ability to combine both the respondent's and their partner's use of violence and control into dyadic typological categories. Using the classification of the respondent and their partner, a combined four-cluster classification was developed accounting for the dyadic nature of the violent and controlling behaviour. All non-violent respondents and partners are classified as non-violent. If an individual (respondent or partner) is violent, he or she is classified as:

1. Intimate terrorism if he/she is high control and his/her partner is either nonviolent or violent but low control;

2. Violent resistance if he/she is low control and his/her partner is violent and high control;

3. Situational couple violence if he/she is low control and his/her partner is either nonviolent or violent and low control; or,

4. Mutual violent control if he/she is high and control and his/her partner is violent and high control.

Where both the respondent and their partner were reported as non-violent, their relationship typology classification was also 'non-violent'.

The first stage of the analysis was to extract the data from Qualtrics and read it in to SAS for data formatting and analysis. All statistical analysis for the quantitative study was done using the statistical computer software SAS v9.1. Within SAS, variables 
were created to calculate relative frequencies and scores for scales. Scales were reverse-scored where necessary.

Descriptive statistics were calculated for the demographic variables to look at the distribution of the survey population and the frequency of reporting for the study measures including physical, psychological and sexual violence. The coercive control measure was dichotomised to create Johnson's (2008) typology of partner violence. Ideally the scale is clustered into two groupings, high and low coercive control. However, depending on the nature of the sample, the cut-off point may differ limiting its comparability to other typological analysis. Johnson performed a cluster analysis on the 7-item Coercive Control Scale for his sample (from the NVAWS; Tjaden \& Thoennes, 1999) that included a "reasonable spread" of intimate terrorism and situational couple violence. Johnson created the clusters using a k-means cluster analysis with a two-cluster solution. The cut-off point was identified as high control, being the reported use of three or more of the seven control tactics while those with two or less control tactics were categorised as low control. To create consistency in developing the typology in this study, Johnson's (2008) cut-off was used. To expand on Johnson's typology and to address potential limitations with his method (See Chapter Three for a discussion on limitations of Johnson's research) the current study included both males and females as respondents. The full analysis and a discussion of these findings can be found in Chapters Five and Six.

A series of chi-square tests were run to test associations between variables. In cases where the chi-square assumption of no more than $20 \%$ of expected counts being less than five was violated, the Fisher exact test was used instead. An alpha level of .05 was used as the significance criterion for these tests. Noting, to avoid issues of multiple testing, I did not carry out all possible tests but rather tested specific hypotheses and presented all results whether significant or not.

\section{QUALITATIVE INTERVIEWS}

With the survey analysis underway I began to organise in-depth interviews with survey participants who agreed to take part in this next stage of the study. The qualitative indepth interviews were conducted between February 2012 and December 2012 to address the second research aim, i.e., to understand the personal stories of young 
people within the context of dating relationships. The qualitative interviews captured the narratives on young adults' understanding of their experience of dating and dating violence in New Zealand and explored the utility of the typology in understanding the complexity of dating violence experiences to assess its potential as a valid framework to understand the different experiences of violence in dating relationships.

I selected a qualitative approach for this study to capture, describe and understand the depth and complexity of the experiences of young people in the context of peers, romantic partners and the wider social context and societal expectations. Such data can provide a greater depth of interaction with participants and allow researchers to look at observable patterns and meaning through narrative forms of data (Heith \& Miller, 2015).

For the in-depth interviews, the focus was on those who had experienced violence in an intimate dating relationship. A participant selection matrix was developed to include a range of experiences and circumstances and participants were selected who represented points along the continuum of violence. The criteria for selection from among those willing to be interviewed included coverage of the spectrum of violent experiences, ensuring representation of the two main categories of Johnson's (2008) typology, intimate terrorism and situational couple violence; student and non-student sample; and males and females.

During the time that the online survey was running, those who provided consent to be contacted for the in-depth interviews were assessed against the participant selection matrix and if they met these criteria were contacted immediately to take part in the study. Contact was made through email and the selected participants were sent the interview information sheet (see Appendix 5) as well as options for times to meet up. Depending on where the participant was recruited (university, polytechnic or crisis support agency) and their location (e.g., outside of Wellington) an appropriate venue was chosen. Online survey participants who were willing to be contacted but did not fit the selection matrix were emailed and thanked for their willingness to participate further but informed that they were not required to do so. They were also reminded of safety information and referred to the website for further queries or information. 
Sixty-nine of the 374 respondents who completed the online survey provided details/were happy to be contacted to take part in the interviews. Forty-three of these 69 respondents were contacted via the email address they provided. Twenty-six respondents were not contacted as they did not provide full information or meet the interview participant matrix. Of 43 respondents that were contacted, 16 were interviewed. This was fewer than the number invited to participate due to some people not being available during the interview period or not responding to email correspondence to arrange an interview time. While this is a small number it is large enough for a qualitative study, and allowed for the responses to get to the point where convergence in experiences was found for a number of topic areas. Most interviews took place at the university, three took place at cafes around Wellington, two were done over the phone and one interview was done via Skype. The interviews lasted an average of 50 minutes. The interviews took place in spaces that supported the safety of the interviewer and the participants but were also places participants felt were private and safe enough to speak about personal and sensitive matters with relative comfort.

As will be discussed further in Chapter Eleven, the sample self-selected into the study and were predominantly, but not exclusively, university students. In this sense the experiences of the social context were largely embedded within the New Zealand university culture. The majority of the participants were also based in Wellington as this was where the recruitment of participants primarily took place, but as discussed in Chapter Eleven the unanticipated consequence of survey respondents sharing the survey link on social media, and snowballing recruitment techniques through key informants meant that some participants lived outside of the Wellington region.

Here, in Table 1, I present the 16 participants using the pseudonyms that are used in the later chapters. The typology classifications in the table below (situational couple violence, intimate terrorism and not violent), age and ethnicity came from the survey analysis detailed in Chapter Five. The other descriptive details were captured during the qualitative interviews. 


\begin{tabular}{ll}
\hline Name & Participant Description \\
\hline Rebecca & Rebecca was a 20-year-old female who identified as New Zealand European. \\
& She described her dating relationship with ex-boyfriend Ben, who was 20 years \\
& old. They had been dating for about two years. She lived in Wellington and was \\
& a second-year student at Victoria University of Wellington. Rebecca was \\
& classified as situational couple violence and her boyfriend as situational couple \\
& violence, and she described minor physical aggression and psychological \\
& violence. High levels of jealousy were described. She described agreeing to \\
& have sex with Ben, even when did not want to. \\
Sarah & Sarah was a 22-year-old New Zealand European female who described her \\
& current dating relationship with Simon, aged 27 years old. They had been \\
& together around 18 months and lived together. Both were classified as \\
& situational couple violence and she described minor violence such as pushing \\
& each other and she would bite him. Sarah also described scenarios where she \\
had used a wine bottle as a weapon. Sarah also talked about frequently getting \\
drunk and being unsure if she had engaged in sexual intercourse with her \\
boyfriend. Sarah also experienced injury that did not require hospital treatment.
\end{tabular}

Sue Sue was a 21-year-old Malaysian female. Her current boyfriend, John, was also Malaysian and was 24 years old. Sue and John had been together for nine years. Sue was classified as non-violent and John was classified as situational couple violence and used minor physical violence that did not result in injury. Sue described strong patterns of control that she described as being part of her culture, she also described a relationship that was traditional and where they did not engage in any sexual activity. John's jealousy and control appeared grounded in strong cultural beliefs on the role of men and women.

Kathryn Kathryn was a 21-year-old Ukrainian woman who described her relationship with her ex-boyfriend Brad. He was New Zealand European and 23 years old. Kathryn was a student and Brad worked full-time. Kathryn was classified as non-violent while Brad was classified as situational couple violence. She described minor violence including psychological violence and pressure to engage in sexual acts to "keep him happy".

Mary Mary was classified as situational couple violence while her ex-boyfriend David was classified as non-violent. She was aged 20 at the time of the interview and 
David was 21 years old. They had been living together during their relationship. She described her use of physical violence and that she caused minor injury. Mary talked about how she pressured David to have sex and threatened to harm herself if he broke up with her.

Emma Emma was 19 years old and identified as New Zealand European. She described her current boyfriend Kyle, he was 20 years old and of New Zealand European decent. Emma worked full time while Kyle was unemployed. They were living together. Emma was classified as non-violent and Kyle was classified as situational couple violence. She described 'play fighting' that was physical in nature and caused minor injury. He also forced her to engage in sexual activity. Emma described his anger that was the result of his unease with her being employed and him not earning money.

\begin{tabular}{|c|c|}
\hline Beth & $\begin{array}{l}\text { Both Beth and her current partner Ethan were classified as situational couple } \\
\text { violence. Beth was a } 21 \text {-year-old university student and Ethan was } 22 \text {-year-old } \\
\text { student. Both were New Zealand European. They lived together. Beth described } \\
\text { how they both pushed and shoved each other and they yelled and called each } \\
\text { other names. }\end{array}$ \\
\hline Drake & $\begin{array}{l}\text { Drake was a 22-year-old male who described his relationship with his ex- } \\
\text { girlfriend Claire. Both were NZ European and university students. Drake was } \\
\text { classified as non-violent and Claire as situational couple violence. He described } \\
\text { her calling him names and using psychological abuse. Drake struggled with } \\
\text { being a male and being a victim and described himself as "pathetic". }\end{array}$ \\
\hline
\end{tabular}

Jack Jack and his ex-partner Pauline were both classified as situational couple violence. He was a 20-year-old student and his girlfriend Pauline was also a student and 19 years old. He described their use of minor physical violence. He normalised coercive sexual experiences. He had also used objects in the home to cause injury.

Grant Grant identified as a young gay man who described his relationship with his exboyfriend Ryan. Grant was a 21-year-old student and Ryan was a 23-year-old 'young professional'. They lived together during their relationship. Grant was non-violent while Ryan was classified as situational couple violence. Grant described physical violence that resulted in minor injury. He also described how his ex-boyfriend threatened to 'out him' through the use of technology and non- 
consented recording of sexual activity.

\begin{tabular}{|c|c|}
\hline Jessica & $\begin{array}{l}\text { Jessica was a } 25 \text {-year-old student who described her relationship with her ex- } \\
\text { boyfriend Zac, who was also } 25 \text { years old. They had been dating for three years. } \\
\text { She was classified as non-violent and he was classified as an intimate terrorist. } \\
\text { The abuse she described included extreme control and escalated to include } \\
\text { severe physical violence, injury from an incident with a knife and him strangling } \\
\text { her. He also coerced Jessica into having sex with him. The impact of Zac's } \\
\text { violence required medical attention and police intervention. }\end{array}$ \\
\hline Sammy & $\begin{array}{l}\text { Sammy was a } 21 \text {-year-old female who had been in a two-year relationship with } \\
\text { her ex-boyfriend Tim. Tim was } 22 \text { years old Albanian, she identified as New } \\
\text { Zealand European. They were both students and lived together while they were } \\
\text { dating. Sammy was classified as non-violent while Tim was classified as an } \\
\text { intimate terrorist. She described his irrational and unpredictable anger, breaking } \\
\text { objects and physical violence, which lead to minor injury. He forced her to have } \\
\text { sex with him and had recorded them during sexual activity without her consent, } \\
\text { which he used to threaten her if she said he thought she was going to leave him. } \\
\text { She described high levels of jealousy and anger issues. }\end{array}$ \\
\hline
\end{tabular}
Karen Karen described a very violent relationship with her ex-boyfriend Craig. He was classified as an intimate terrorist while Karen classified as non-violent. Karen was aged 22 years, a university student and New Zealand European. Craig was also 22 years, a student and originally from Australia. Karen described how he was physically violent and was scary as he "flipped between being a gentleman and a monster". She experienced forced sexual activity. She experienced minor injury but did not seek medical attention.

Laura Laura was a 23-year-old New Zealand European woman who was employed full-time. Her ex-boyfriend Harry was a 24-year-old Māori man who was unemployed. She was classified as non-violent and he was classified as an intimate terrorist. Laura described severe violence and surveillance tactics that resulted in injury. He was highly controlling and unpredictable and had worked to isolate her from her friends and family. She described the ever-changing rules that created uncertainty in how he would react to her.

Ricky Ricky was a 25-year-old Māori man who described his relationship with his onagain off-again ex-partner Jane. He was employed but in sporadic, seasonal 


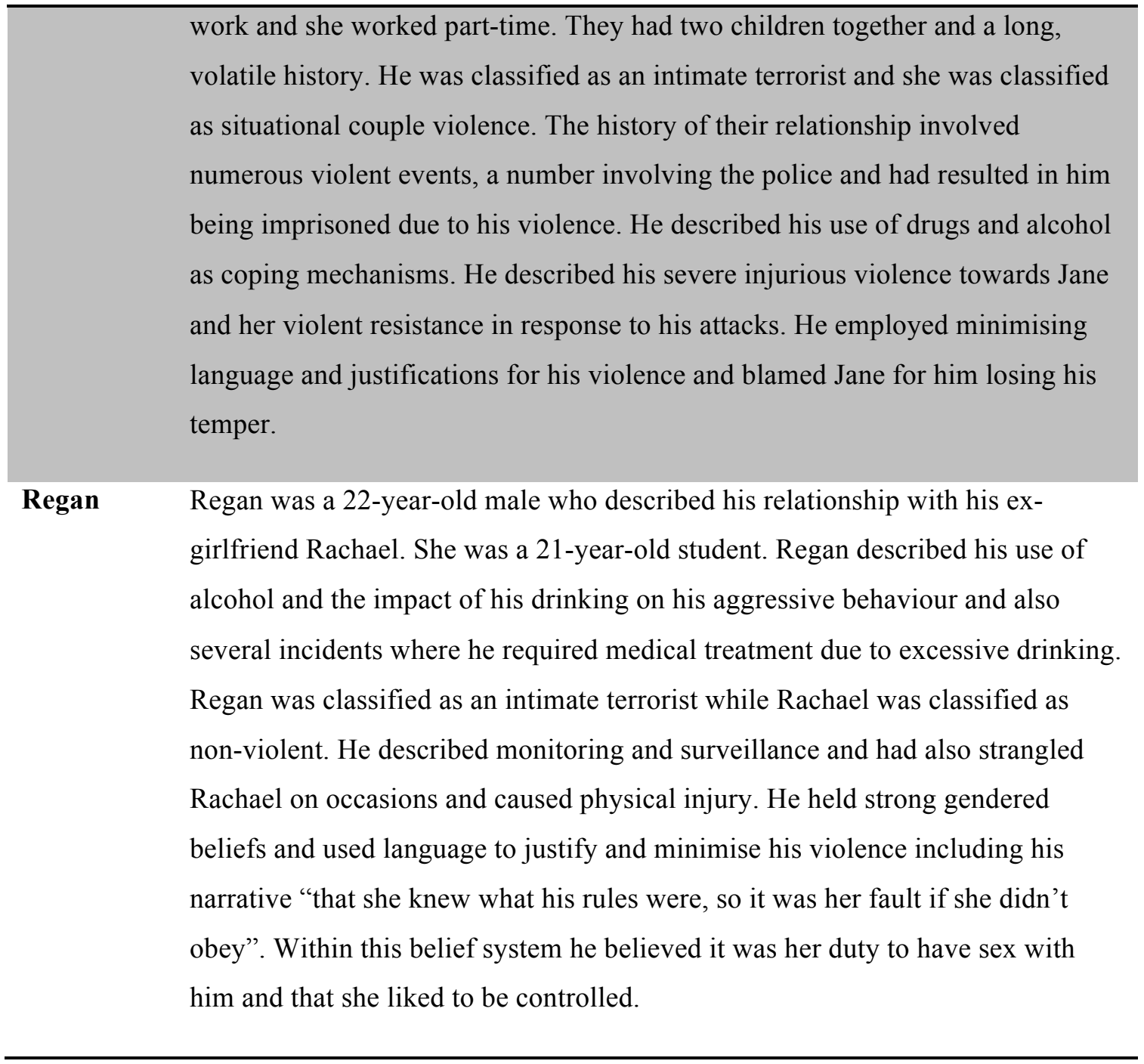

I developed a semi-structured interview guide based on the findings from the review of the literature, the key informant interviews, and the online survey. The semi-structured participant-led approach allowed the conversation to move in and out of the personal and general discussion depending on how the interviewee led the discussion and how comfortable they felt talking about their experiences. It allowed for the participants' flow of thoughts and a more natural conversation-style approach to be followed, left room for emergent themes and made the process more 'participant-driven' rather than primarily 'researcher-driven' (McIntosh \& Morse, 2013). The interview guide was not rigid but was there to assist me to cover key areas and to have prompts for key questions if necessary. See Appendix 5 for the interview guide.

Five topic areas were identified through the literature review, key informant interviews and through the online survey. Within each topic area a range of questions was 
developed that would prompt the interviewer to promote conversation and help the interview flow. These topic areas included the current social context of dating and relationships for young adults in New Zealand; participant's experience of dating; experience of dating violence; understanding of dating violence (actual and perceived); and the impact of dating violence.

The format of the interviews was as follows: I began by introducing myself and thanking the participant for coming in for the interview, I explained the goals of the project, provided the information sheet, obtained informed consent from the participant (a copy of which can be found in Appendix 7), and asked for the participant's permission to record the interview (I explained how the recording would be used, how it would be protected/secured and when the recording would be destroyed).

Once consent was obtained the digital recording began. Rather than starting the interviews with discussions of violence that could feel threatening and uncomfortable for participants, I built rapport by asking participants a little bit about themselves, for example where they were from, how they found out about the survey and their current situation. This led into questions related to the social context of violence. These questions included the participant's ideas of what dating means, what is acceptable or not in a dating relationship, and what relationships look like when they are healthy or not.

The interview then moved into the 'formal' topic areas addressing personal experiences of dating and violence. Several strategies were used to assist the participants in telling their story. These included asking the participant to describe their experience of dating including what the culture around dating is in New Zealand; discussing 'hooking-up' and meeting people, the establishment of relationships and how these relationships 'typically' progress; discussing negative experiences in relationships and specific violent events; and asking for their thoughts on power and control and healthy and unhealthy relationships.

I was cognisant of the potential for these young adults to want to sanitise or minimise their experiences of control, conflict and violence. Most participants were clear that they had not been in a violent relationship, but as they became more comfortable through the interview they began to share more about the negative experiences they 
had encountered. I was very deliberate about not directly asking the interviewees of their experience of violence or to imply a judgement on what was 'bad' so I framed the questions about positive and negative experiences in their relationships. I did not want to close down the conversation or to apply a pre-determined understanding or definition of what dating violence is - the narratives were a way to understand the lived experiences of the participants. I was quite surprised with how open the participants were in their descriptions of their dating experience, views on sex and their engagement in the hook-up culture and night-time economy.

I also wanted to ensure that the interviews concluded with conversations of a more positive nature, particularly if the discussions had focused on negative or concerning experiences. Since some discussions did cover distressing and sensitive topics, it was important to consider participants' well-being and end the interview reflecting on the more positive elements of our discussion. However, all those interviewed were provided with details of support services in case they were required. The participants were thanked for their time and given a \$20 supermarket voucher as koha (gift) in recognition of their time and willingness to disclose their personal stories.

As a way to follow up further and check on any impact from discussing their experiences with me, within a day of the interview I emailed the participants to thank them for their contribution to the research and, again, provided them with links to support agencies. This email also reminded them that they could withdraw from the study at any time and request that their information not be used, that they could follow up with any questions or comments, and I confirmed they would receive a copy of the study if they had previously stated their interest.

\section{Positionality}

Before I continue with detailing my qualitative analysis process, it feels appropriate to disclose my own upbringing and views that potentially influenced the framing and focus of this study and my analysis.

It is well established that it is rather naïve to assume the idea of pure objectivity in research analysis, particularly in qualitative studies within the social sciences. The notion of objectivity comes from the physical and natural sciences and was held up by positivists as a goal to aspire to in social science research. However subsequent 
criticisms have shown it to be impossible to achieve; any research conducted by humans reflects their subjectivities to some extent whether that be in selecting the area they choose to study, how they approach their research or their interpretation of their data (Norman \& Lincoln, 2000; Fonow \& Cook, 2005). Recognising the subjectivities involved in conducting qualitative research has led to considerations of reflexivity, which:

means turning of the researcher lens back onto oneself to recognize and take responsibility for one's own situatedness within the research and the effect that it may have on the setting and people being studied, questions being asked, data being collected and its interpretation. As such, the idea of reflexivity challenges the view of knowledge production as independent of the researcher producing it and of knowledge as objective. (Berger, 2015, p. 220)

In other words, researchers ought to be aware of how they position themselves in the research and to detail how that experience may shape the research and analysis that is presented:

consequently, researchers need to increasingly focus on self-knowledge and sensitivity; better understand the role of the self in the creation of knowledge; carefully self-monitor the impact of their biases, beliefs, and personal experiences on their research; and maintain the balance between the personal and the universal. (Berger, 2015, p. 220)

One step to foster reflexivity is to describe how experiences, beliefs, values and lived assumptions of the researcher may play out through the research process. Mauthner and Doucet (2003) described the importance of "being reflexive about how we interpret our data, our role in the analytic process, and the pre-conceived ideas and assumptions we bring to our analysis" (p. 417). Or in Charmaz and Belgrave's (2019) words "it entails learning to recognize how our worldviews, language, meanings, and unearned privileges enter our research in tacit ways as well as examining ourselves in the research process" (p. 750). Therefore, while sharing my experience as a young adult and of dating is perhaps somewhat indulgent, it is the lens that I bring to understanding and interpreting the narratives collected in this study. 
I lived a relatively 'sheltered' life as a young adult. I grew up in a Christian household where there was no alcohol and strong beliefs prevailed on the role of marriage and sex within the confines of marriage. I spent my university years studying and working - I did not engage in the night-time economy or the wider university culture of socialising and partying. I met my husband when I was 14 years old, we started dating when I was 19 years old and were married in 2006 when I was 25 years old. I lived at home until we were married and at that point I was working as a research and evaluation analyst in the New Zealand public service. My experience of dating and violence was in stark contrast to a majority of the young people I talked to in this study; I had only experienced 'healthy' relationships, no violence or control and stood outside of their experiences of drinking and casual sex.

I had spent most of my teenage years working with 'at-risk' youth and 'deserving kids' (children from violent families and families living in severe poverty), and went overseas on a church mission to work with disadvantaged communities and children and young people with severe and chronic disabilities. I developed a strong empathy and ability to see others' experiences for what they were without much judgement. The ability to be able to connect to stories of violence was also strengthened through my experience as a public servant - interviewing victims and perpetrators of family violence, working closely with youth offenders, reviewing cases of abuse of children in state care and working with young adults in the broader social system. This experience was alongside working with experienced advocates and academics and helped to broaden my understanding of these experiences, to make sense of them (as much as you can) and to reconcile my experiences with those of victims and perpetrators of relationship violence.

To perhaps complicate matters, at the time of conducting the interviews I had just become a mother. My first daughter was just a few months old at the time, and as I will discuss in my reflection in Chapter Eleven, the reality of what my own daughter's future experiences might be hit home. Perhaps I brought my own concern for what she might be exposed to in years to come as I talked to the young women and men, but this new role as a mother also further fuelled my motivation to understand and support learnings in the field of dating violence to afford the generations to come the best 
opportunity to experience healthy relationships and a positive journey through young adulthood.

\section{ANALYTIC APPROACH}

I will now move on to discuss the qualitative analysis process that I employed as I worked through the sense making of the interviews that I conducted. As I collected the interview data, I began to identify themes as they emerged, and these also helped to refine or identify areas of focus for subsequent interviews. This analysis was done using Grounded Theory. Sociologists Glaser and Strauss (1967, in Keddy, Sims, \& Stern, 1996) developed grounded theory in the early 1960s, partially in response to certain schools of thought which questioned the validity of qualitative methods as more sophisticated quantitative methods emerged. This also led to dispute about the validity of qualitative research through the analysis of participant meanings (Charmaz, 2006). To address this tension, Grounded Theory provides a framework to inform qualitative analysis, which Glaser and colleagues argued provided control through the research process and increased the power of the analysis undertaken. Grounded Theory has since been adopted and the basic notions and steps of the research process are adapted to provide diversity in study approaches and findings (Charmaz, 2006).

Grounded theory is an iterative, comparative, and interactive method that begins with inductive data. We grounded theorists analyse our data using comparative methods from the beginning to the end of the research process. (Charmaz \& Belgrave, 2019, pp. 743-44)

There are several stages of analysis within Grounded Theory. Following the capture of rich data, the next step is to develop a set of themes that are important to the participants - through 'open coding' or 'line-by-line' coding of the transcripts. Throughout this process the researcher is looking for similarities and differences between descriptive categories (Burck, 2005). Next these themes are clustered into higher-level categories that are more conceptual or interpretive. The data are then reexamined against these categories to extend these concepts. These higher-level categories are then linked to "theoretical memos" where ideas are explored and synthesised to create a core category that explains the links between these categories this is where the theory is generated in the form of a model or a typology. 
Throughout the data collection phase I reflected at the end of each interview on what I had heard and the key areas that struck me as important to capture. I made notes about my first impressions and used this to refine the interview guide, to identify areas I would want to probe more about in subsequent interviews, and to start identifying significant themes.

As we learn how our research participants make sense of their experiences, we begin to make analytic sense of their meanings and actions. (Charmaz, 2006, p. 11)

I also personally transcribed each interview. This was a time-consuming process. I was not fast at typing but, rather than outsourcing this part of the process, I knew that it was invaluable for me to work through the recordings. Doing so took/transported me back to the interview and enabled me to pick up more of the nuanced language and descriptions that the young people, often quite eloquently and starkly, expressed.

In the interests of my personal well-being, I was deliberate about when I worked on this task. As I re-lived some of the more distressing narratives of violence and harmful behaviours, I made sure that I enacted self-care techniques including transcribing away from my young child; engaging when I knew I could make contact with my supervisor; and, ensuring that I had time away from the process so that I did not become completely consumed by the narratives.

As I carried out the interviews my initial thematic analysis began to converge - by the sixteenth interview I felt I had captured a strong saturation in themes that would provide the insight and understanding needed for this study. I was left with a large number of themes, similar to Charmaz's memo writing in that key codes and themes were created and compared, grouped and solidified into more analytical elements that became the dominant or headline themes in the study, with lower level memos or evidence that sat under the headlines (Charmaz, 2006). As I worked through writing up the results, the overarching key findings and themes became clearer with other findings categorised as sub-themes, and some deemed irrelevant.

There were different iterations on how I chose to present these findings. I began with just one chapter on violence but the depth of information that I had collected needed to be represented further, therefore the analysis ended up spanning three chapters. The 
first of the three qualitative chapters described the social context, norms and expectations that the young adults described. The second focused on the experience of dating violence. The third qualitative chapter described the underlying attitudes and beliefs that were far more overwhelming and consistent across this study than I had anticipated and thus warranted their own chapter. The need to shift attitudes and beliefs was reinforced through the young adults' narratives and is discussed more in Chapter Eleven.

The full analysis and a discussion of these interview findings can be found in Chapters Seven to Nine.

\section{SAFETY AND ETHICS}

This study involved research with human subjects about very sensitive issues and experiences, so careful consideration of ethical issues was taken. This included submission of an ethics application to Victoria University of Wellington's Social and Cultural Studies Human Ethics Sub-Committee. Ethical approval was granted in November 2010 (Ethics Number 17969; see Appendix 2).

For all participants, in both the online survey and in-depth interviews, consent was required before data were collected. An information sheet was provided to participants so they knew that they were participating by choice, they understood what the data were for and how they would be used (survey analysis, key themes, case studies, confidential with no identifying information used), and that they did not have to answer questions they were not comfortable with. Throughout the online survey, participants were reminded that they could exit the survey at any point. This was important as they may have felt distress in answering the questions due to their sensitive nature and especially as participants may be/have been victims or perpetrators of dating violence or wider family violence. If participants chose to exit from the survey before they completed it, they were directed to a page with a list of agencies, resources and information about dating violence should they need to contact someone. The online survey participants were also informed that if they did stop the survey partway through without submitting their responses, their data would not be used in any analysis. However, once participants submitted their data they could not request its 
deletion, as the data was anonymous. Incomplete surveys were not included in any analysis.

When participants finished the online survey, they were asked if they were willing to be contacted for an in-depth interview. Before giving consent, participants were told what the interviews would cover, how long the interview would take and that any information filled out on the online survey would be matched to them by the interviewer to provide context for the in-depth interview (meaning that their online data would no longer be anonymous).

Those who participated in the online survey were required to tick a box to state that they had read the consent details and that they were willing to continue with the survey (they were not able to start the survey if they did not tick the 'accept' button). For those participating in the in-depth interviews, the information sheet highlighted that they could cease participation in the interview at any time, and they could choose for their information not to be included in the study. A written consent form was given to participants at the beginning of the in-depth interview and I talked it through with them. If they agreed be interviewed, they were asked to sign the consent form before the interview began and were provided with a copy of the form and information about the study to take with them. Participants were also able to bring a support person (family, whānau, friend or counsellor) to their in-depth interview if they felt this would help them through the interview process.

The online survey and in-depth interviews included questions about participants' past and current intimate relationships and the occurrence and nature of their personal experiences of violence. Research with victims of partner violence (and other sensitive subjects) suggests that involvement in such studies can have therapeutic value as victims and perpetrators become more aware of their situation (Temple, Weston, \& Marshall, 2005). However, participating in such a study and relaying their traumatic experiences could potentially cause harm to the participants if it resulted in them feeling re-victimised. To help mitigate the risk of harm to participants, prior to the start of interviewing and throughout the interviewing process, respondents were reminded that they did not have to answer all the questions and could stop the interview at any time they wished. At the end of the interviews, respondents were provided with a list 
of local agencies and services including the 0800456450 National Family Violence Information Line.

Participants in this study could be perpetrators or victims of violence. To ensure safety for both the interviewer and participants, careful consideration was taken as to where the interviews took place (for example location, visibility and time of the day).

\section{CHAPTER SUMMARY}

This chapter outlined the mixed methods approach used to answer the study research questions. The phases of the data collection provided me with constant learning and reflection as I developed my focus and findings. The interviews with my key informants allowed me access to a wealth of knowledge, experiences and perspectives grounded in the New Zealand context. Throughout the data collection and analysis I was constantly challenged in my own positioning and had to broaden my views on young adults' attitudes and engagement in the night-time economy of drinking to excess and casual sex and assess my reactions to this as being negative and harmful. This was challenged, as I learned more about the culture of young adults, particularly those engaging in student/university culture. It was also sometimes distressing listening to the stories of young women and men describing their violent relationship and the trauma they had already experienced in their lives.

In the following chapters I provide a detailed discussion of the findings from the quantitative online survey (Chapters Five and Six) and the qualitative in-depth interviews (Chapters Seven, Eight and Nine); and a synthesis and discussion of the findings across this thesis, weaving together the strands of data including the literature review, survey analysis and interview findings (Chapter Ten). 


\title{
Chapter Five - EXPERIENCE OF DATING VIOLENCE, IS IT REALLY THAT COMMON?
}

\author{
IPV is a serious and pervasive problem in New Zealand. It is not limited to any \\ gender, cultural, economic, or religious group. (Robertson \& Murachver, \\ 2007a, p. 639)
}

In this chapter I will present the descriptive analysis from the quantitative study. As discussed in Chapter Four, this analysis aims to measure the level of violence experienced by young adults in dating relationships in New Zealand through the quantitative online survey. I begin firstly with a description of the sample that took part in this survey followed by the reported frequency of dating violence for this sample of young people and end with statistics on the levels of injury reported by participants as a result of dating violence.

The data from this study came from an online survey that examined young adults' experiences of violence, including psychological aggression, physical assault and sexual coercion. The analysis was based on 374 respondents who met the study criteria and completed the survey (see Chapter Four for a detailed description of the survey methodology employed in this study). A series of significance tests were run using chisquare and Fisher exact tests. An alpha level of .05 was used as the significance criterion for these tests.

\section{SECTION ONE: DEMOGRAPHICS}

A number of demographic variables were collected about both the respondent and their current or ex-partners. Table 2 summarises this self-reported information and includes the distributions across categories for the respondent and for the partner that they reported on. The following section details these results.

The study yielded a total of 314 female (84\%) and 60 male (16\%) respondents who met the study criteria. Smith (2008) writes that it is common for there to be an overrepresentation of females participating in self-selected research. The majority of these young adults reported being in a heterosexual relationship $(n=345,92.2 \%)$. Of these, 294 were females who responded about a current $(n=238)$ or ex- $(n=56)$ relationship 
with a male partner. Fifty-one respondents were males with 46 responding about a current female partner and five responding about an female ex-partner. The remaining 29 respondents $(7.8 \%)$ reported being in a same-sex relationship. Twenty females described a current $(n=11)$ or ex- $(n=9)$ relationship with a female, while nine males described a current $(n=7)$ or ex- $(n=2)$ relationship with a male.

Respondents' ages ranged from 18 to 25 years old with a mean age of 20.5 years. Nearly three-quarters of the sample were aged $18-21$ years old (71.1\%). Only $28.9 \%$ of respondents were 22-25 years old. A slightly different pattern was found for the age of respondents' partners, with $57.2 \%$ of participants reporting their partner's age as $18-21$ years old, $30.7 \%$ were aged $21-25$ years, while $12.0 \%$ of partners were reported to be over the age of 25 . The reason for pattern in age distribution for respondents' partners was that while respondent selection criteria into the study included only those aged 1825 years old, there was no restriction on the age of their partners.

The majority of the sample reported their ethnicity as New Zealand European (74.1\%) and their partner's ethnicity also as New Zealand European (71.7\%). Less than 10\% (9.4\%) of respondents identified as Māori, with 16.6\% classified as 'other'. 
TABLE 2 DEMOGRAPHIC CHARACTERISTICS FOR RESPONDENTS AND THEIR PARTNERS

\begin{tabular}{|c|c|c|c|c|}
\hline & \multicolumn{2}{|c|}{ Respondents } & \multicolumn{2}{|c|}{ Partners } \\
\hline & Frequency & Percentage & Frequency & Percentage \\
\hline \multicolumn{5}{|l|}{ Gender } \\
\hline Female & 314 & $84.0 \%$ & 71 & $19 \%$ \\
\hline Male & 60 & $16.0 \%$ & 303 & $81.0 \%$ \\
\hline \multicolumn{5}{|l|}{ Age } \\
\hline $18-21$ years & 266 & $71.1 \%$ & 214 & $57.2 \%$ \\
\hline $22-25$ years & 108 & $28.9 \%$ & 115 & $30.7 \%$ \\
\hline Over 25 years & $\mathrm{N} / \mathrm{A}^{2}$ & & 45 & $12.0 \%$ \\
\hline \multicolumn{5}{|l|}{ Ethnicity } \\
\hline NZ European & 277 & $74.1 \%$ & 268 & $71.7 \%$ \\
\hline Māori & 35 & $9.4 \%$ & 23 & $6.1 \%$ \\
\hline Other Ethnicity & 62 & $16.6 \%$ & 83 & $22.2 \%$ \\
\hline \multicolumn{5}{|l|}{ Highest qualification } \\
\hline Any school qualification & 200 & $53.5 \%$ & & \\
\hline $\begin{array}{l}\text { Bachelor degree or Post- } \\
\text { graduate degree }\end{array}$ & 127 & $34.0 \%$ & & \\
\hline $\begin{array}{l}\text { Diploma, certificates, } \\
\text { others }\end{array}$ & 47 & $12.6 \%$ & & \\
\hline \multicolumn{5}{|c|}{ Source of income (the previous 12 months) } \\
\hline No income & 19 & $5.1 \%$ & & \\
\hline Wage, salary & 176 & $47.1 \%$ & & \\
\hline $\begin{array}{l}\text { Student allowance, other } \\
\text { benefit }\end{array}$ & 159 & $42.5 \%$ & & \\
\hline Other source of income & 20 & $5.3 \%$ & & \\
\hline \multicolumn{5}{|c|}{ Total income (the previous 12 months) } \\
\hline$\$ 1-\$ 10,000$ & 198 & $52.9 \%$ & & \\
\hline$\$ 10,001-\$ 40,000$ & 128 & $34.2 \%$ & & \\
\hline$\$ 40,001$ or more & 21 & $5.6 \%$ & & \\
\hline No income & 5 & $1.3 \%$ & & \\
\hline Refused & 22 & $5.9 \%$ & & \\
\hline
\end{tabular}

${ }^{2}$ Survey respondents were screened out of the survey if they were over 25 years old. 
This distribution of ethnicity is similar to other New Zealand-based research on IPV. For example, Robertson and Murachver (2007b) conducted a study that included university students, a general population sample and incarcerated participants. Of those in the student population 4.5\% identified as Māori, 3\% identified as Pacific and 83.6\% of students identified as Pākehā $\bar{a}^{3}$. Similarly, Jackson (1998a) conducted a study on dating violence with 373 senior high school students aged 16-20 years and reported that $8.4 \%$ of the sample reported their ethnicity as Māori while $9.4 \%$ of students reported their ethnicity as Pacific. This under-representation of Māori and other ethnic groups in the current study limits the generalisability to the wider New Zealand population; the implications are detailed in Chapter Four.

Respondents were asked questions about their highest qualification. Table 2 shows that $53.5 \%$ of respondents listed their highest qualification as "any school qualification", although $75.7 \%$ of respondents were currently enrolled at Victoria University of Wellington, Whitireia (1.9\%), WelTec (1.3\%) or "other" educational institution $(7.8 \%)$. Respondents were also asked what their main source of income was in the previous 12 months. Just under half the respondents reported their income came from "wages, salary, commissions, bonuses, etc, paid by my employer" (47.1\%), with 159 respondents $(42.5 \%)$ receiving a student allowance or other government benefit. Five percent of the sample received no income during this time. Respondents were also asked what their total income was before tax in the previous 12 months. Half of respondents $(52.9 \%)$ earned $\$ 10,000$ or less during that period.

Participants were also asked how they found out about the survey. Just under half of respondents were recruited through in-class methods while only $12.8 \%$ of the sample reported being recruited through an agency. In Chapter Four there is a discussion of the limitations of this variable. In summary, the recruitment process for participants that engaged with crisis support agencies was altered and participants were recruited through key informants and snow-balling techniques. The implications for the survey were that response categories could not capture clients of agencies as such, and some were likely to have responded to hearing about the study through "word of mouth".

\footnotetext{
${ }^{3}$ Pākehā is the term used to describe people of European descent living in New Zealand.
} 
This category will therefore include those who were recruited through key informants as well as participants who found out about the survey from other participants (e.g., through Facebook).

Table 3 shows that nearly all of the respondents reported being in a dating relationship $(96.5 \%)$ with only $3.5 \%$ reporting that they were engaged. Four-fifths of respondents reported being in a current relationship $(80.7 \%$ ) while $19.3 \%$ of respondents responded to the survey about a past relationship.

Nearly half of the respondents reported that they were in a relationship that lasted three to $11^{4}$ months $(44.9 \%)$. Those who were in current relationships were more likely to be in a longer lasting relationship, with $28.8 \%$ in relationships that had lasted between one and two years compared to no-one reporting on a past relationship; and 31.1\% reported a current relationship that had lasted from two to four years compared to $4.2 \%$ of those who reported a past relationship. Only 22 respondents had been in a relationship that lasted four years or more and they were all still currently with that partner.

The majority of respondents reported that they lived in a flatting situation without their partner (59.9\%), while nearly one-third of respondents (29.4\%) lived with their partner whether that be in a flatting situation, in their own dwelling or with parents. A further $10.7 \%$ of respondents lived with a parent in their family home.

Data from this study were collected through young adults who self-selected into the study and the above sample is not representative of young adults in New Zealand, therefore prevalence scores could not be calculated in this analysis. As a result, the analysis in the remaining chapters should be understood in the context of the sample that participated in the study. The limitation and implications of the sample will be discussed in the final chapter, Chapter Eleven.

\footnotetext{
${ }^{4}$ Participants were excluded from the survey if they had not had a current or recent relationship that had lasted 3 months or more.
} 
TABLE 3 CHARACTERISTICS OF THE RELATIONSHIP

\begin{tabular}{|c|c|c|}
\hline \multirow[t]{2}{*}{ Characteristics of respondents } & \multicolumn{2}{|c|}{ Participants } \\
\hline & Frequency & Percentage \\
\hline \multicolumn{3}{|l|}{ Current or ex-partner } \\
\hline Current partner & 302 & $80.7 \%$ \\
\hline Ex-partner & 72 & $19.3 \%$ \\
\hline \multicolumn{3}{|l|}{ Sexual orientation } \\
\hline Heterosexual & 346 & $92.5 \%$ \\
\hline Same-sex & 28 & $7.5 \%$ \\
\hline \multicolumn{3}{|l|}{ Relationship status } \\
\hline Dating & 361 & $96.5 \%$ \\
\hline Engaged & 13 & $3.5 \%$ \\
\hline \multicolumn{3}{|l|}{ Length of relationship } \\
\hline $3-11$ months & 168 & $44.9 \%$ \\
\hline $12-23$ months & 87 & $23.3 \%$ \\
\hline $24-47$ months & 97 & $25.9 \%$ \\
\hline 4 years or more & 22 & $5.9 \%$ \\
\hline \multicolumn{3}{|l|}{ Living status } \\
\hline $\begin{array}{l}\text { Live in the family home - not with } \\
\text { partner }\end{array}$ & 40 & $10.7 \%$ \\
\hline Live with flatmates - not with partner & 224 & $59.9 \%$ \\
\hline Living with partner & 110 & $29.4 \%$ \\
\hline \multicolumn{3}{|l|}{ How they found out about survey } \\
\hline Through an agency & 48 & $12.8 \%$ \\
\hline In class & 184 & $49.2 \%$ \\
\hline Social media / Word of mouth & 142 & $38.0 \%$ \\
\hline
\end{tabular}

\section{SECTION TWO: EXPERIENCE OF VIOLENCE - AS VICTIMS AND AS PERPETRATORS}

This section presents findings on the frequency of violence experienced by respondents, both as a victim and/or perpetrator of dating violence. Analysis includes overall counts of dating violence; frequency of different types of violence including psychological aggression, physical assault and sexual coercion; the type of conflict tactics used; and counts for minor and severe violent experiences. 
Respondents' experiences of dating violence were measured through the CTS2 (Straus et al., 1996). As detailed in Chapter Four, the CTS2 asks respondents how often they had experienced certain behaviours as a victim and/or perpetrator in the previous 12 months. In this study respondents answered these questions about the current or expartner who was identified at the start of the survey. Further analysis in this chapter will look at other factors alongside the CTS2 score including severity of the violence; number of acts of violence used; the types of violence experienced; and experience of injury, to add more context to this analysis and the experience of respondents.

\section{FREQUENCY OF VIOLENCE - OVERALL COUNT}

Table 4 summarises the frequency of violence in the previous 12 months experienced by both victim and perpetrator. Respondents who reported experiencing one or more acts on the CTS2 were classified as a victim of violence, and where respondents reported engaging in one or more acts on the CTS2 were labelled as a perpetrator. The same classification was applied to their current or ex- dating partner.

TABLE 4 THE NUMBER OF RESPONDENTS WHO HAVE EXPERIENCED VIOLENCE BETWEEN AN INTIMATE PARTNER OVER THE PREVIOUS 12 MONTHS

\begin{tabular}{|c|c|c|c|}
\hline & Total & Females & Males \\
\hline Respondents victimisation from violence & $\mathbf{2 9 0}$ & 245 & 45 \\
& $\mathbf{7 7 . 5 \%}$ & $78.0 \%$ & $75.0 \%$ \\
\hline \multirow{2}{*}{ Respondents perpetration of violence } & $\mathbf{2 9 8}$ & 251 & 47 \\
& $\mathbf{7 9 . 7} \%$ & $79.9 \%$ & $78.3 \%$ \\
\hline
\end{tabular}

Results in Table 4 show that the majority of respondents experienced one or more acts of violence in the previous 12 month (77.5\%), with 78.0\% of female respondents and $75.0 \%$ of male respondents reporting being a victim of one or more acts from the CTS2. Similarly, this study found that the majority of respondents (four-fifths or 79.7\%) reported perpetrating one or more acts from the CTS2 against their dating partner in the previous 12 months, representing $79.9 \%$ of female respondents and $78.3 \%$ of male respondents.

Chi-square analysis was conducted for victimisation and perpetration to investigate whether the differences in reporting violence by gender were significant. The results showed that there were no significant differences between the number of male and female respondents who reported experiencing one or more acts on the CTS2, both as 
victims $\left(\chi^{2}(1)=.120, p=.730\right)$ and perpetrators $\left(\chi^{2}(1)=.012, p=.914\right)$. These results suggest gender symmetry in the experience of violence in young adult relationships.

These findings are consistent with other studies, for example in Archer's (2000) metaanalysis of dating violence he reported that both sexes reported perpetrating violence, and he also concluded overall women were actually more likely than men to use violence, which is consistent with this study where women also reported slightly higher rates of perpetrating violence against their dating partner.

\section{DistribUtion OF RESPONSE TO EACH CTS2 ITEM}

The CTS2 has a number of scales made up of minor and severe conflict tactic items.

Table 5 shows the CTS2 scale items used to measure experiences of violence and the frequency of responses across the range of items.

The distribution of responses is seen across all items of the CTS2, with a greater frequency of responses for minor items such as "I swore at my partner"; "I shouted or yelled at my partner"; "I shoved my partner". However, there also was coverage of responses across the severe items of the CTS2, especially across psychological violence such as "I called my partner fat and ugly"; "I threatened to hit or throw something at my partner"; and physically aggressive acts such as "I punched or hit my partner with something that could hurt".

TABLE 5 THE DISTRIBUTION OF RESPONSES TO EACH OF THE CTS2 ITEMS OVER THE PREVIOUS 12 MONTHS

\begin{tabular}{|l|l|c|c|c|c|}
\hline & & \multicolumn{2}{|c|}{$\begin{array}{c}\text { Respondents } \\
\text { victimisation from } \\
\text { violence }\end{array}$} & \multicolumn{2}{|c|}{$\begin{array}{c}\text { Respondents } \\
\text { perpetration of } \\
\text { violence }\end{array}$} \\
\hline & & Frequency & $\%$ & Frequency & $\%$ \\
\hline Psychological aggression & \multicolumn{5}{|l|}{$\%$} \\
\hline I insulted or swore at my partner & Minor & 231 & $61.8 \%$ & 253 & $67.6 \%$ \\
\hline I shouted or yelled at my partner & Minor & 174 & $46.5 \%$ & 194 & $51.9 \%$ \\
\hline $\begin{array}{l}\text { I stomped out of the room or } \\
\text { house or yard during a } \\
\text { disagreement }\end{array}$ & Minor & 147 & $39.3 \%$ & 208 & $55.6 \%$ \\
\hline $\begin{array}{l}\text { I said something to spite my } \\
\text { partner }\end{array}$ & Minor & 143 & $38.2 \%$ & 108 & $28.9 \%$ \\
\hline I called my partner fat or ugly & Severe & 64 & $17.1 \%$ & 36 & $9.6 \%$ \\
\hline $\begin{array}{l}\text { I destroyed something belonging } \\
\text { to my partner }\end{array}$ & Severe & 34 & $9.1 \%$ & 22 & $5.9 \%$ \\
\hline I accused my partner of being a & Severe & 38 & $10.2 \%$ & 52 & $13.9 \%$ \\
\hline
\end{tabular}




\begin{tabular}{|c|c|c|c|c|c|}
\hline lousy lover & & & & & \\
\hline $\begin{array}{l}\text { I threatened to hit or throw } \\
\text { something at my partner }\end{array}$ & Severe & 38 & $10.2 \%$ & 38 & $10.2 \%$ \\
\hline \multicolumn{6}{|l|}{ Physical assault } \\
\hline $\begin{array}{l}\text { I threw something at my partner } \\
\text { that could hurt }\end{array}$ & Minor & 40 & $10.7 \%$ & 33 & $8.8 \%$ \\
\hline $\begin{array}{l}\text { I twisted my partner's arm or } \\
\text { hair }\end{array}$ & Minor & 41 & $11.0 \%$ & 19 & $5.1 \%$ \\
\hline I pushed or shoved my partner & Minor & 69 & $18.4 \%$ & 80 & $21.4 \%$ \\
\hline I grabbed my partner & Minor & 59 & $15.8 \%$ & 49 & $13.1 \%$ \\
\hline I slapped my partner & Minor & 47 & $12.6 \%$ & 64 & $17.1 \%$ \\
\hline $\begin{array}{l}\text { I used a knife or gun on my } \\
\text { partner }\end{array}$ & Severe & 9 & $2.4 \%$ & 2 & $0.5 \%$ \\
\hline $\begin{array}{l}\text { I punched or hit my partner with } \\
\text { something that could hurt }\end{array}$ & Severe & 36 & $9.6 \%$ & 20 & $5.3 \%$ \\
\hline I choked my partner & Severe & 20 & $5.3 \%$ & 8 & $2.1 \%$ \\
\hline $\begin{array}{l}\text { I slammed my partner against a } \\
\text { wall }\end{array}$ & Severe & 23 & $6.1 \%$ & 12 & $3.2 \%$ \\
\hline I beat up my partner & Severe & 20 & $5.3 \%$ & 4 & $1.1 \%$ \\
\hline $\begin{array}{l}\text { I burned or scalded my partner } \\
\text { on purpose }\end{array}$ & Severe & 3 & $0.8 \%$ & 2 & $0.5 \%$ \\
\hline I kicked my partner & Severe & 25 & $6.7 \%$ & 22 & $5.9 \%$ \\
\hline \multicolumn{6}{|l|}{ Sexual coercion } \\
\hline $\begin{array}{l}\text { I made my partner have sex } \\
\text { without a condom }\end{array}$ & Minor & 58 & $15.5 \%$ & 31 & $8.3 \%$ \\
\hline $\begin{array}{l}\text { I insisted on sex when my } \\
\text { partner did not want to (but did } \\
\text { not use physical force) }\end{array}$ & Minor & 89 & $23.8 \%$ & 55 & $14.7 \%$ \\
\hline $\begin{array}{l}\text { I insisted my partner have oral } \\
\text { or anal sex (but did not use } \\
\text { physical force) }\end{array}$ & Minor & 38 & $10.2 \%$ & 20 & $5.3 \%$ \\
\hline $\begin{array}{l}\text { I used force (i.e., hitting, holding } \\
\text { down, or using a weapon) to } \\
\text { make my partner have oral or } \\
\text { anal sex }\end{array}$ & Severe & 7 & $1.9 \%$ & 1 & $0.3 \%$ \\
\hline $\begin{array}{l}\text { I used force (like hitting, } \\
\text { holding down, or using a } \\
\text { weapon) to make my partner } \\
\text { have sex }\end{array}$ & Severe & 17 & $4.5 \%$ & 2 & $0.5 \%$ \\
\hline $\begin{array}{l}\text { I used threats to make my } \\
\text { partner have oral or anal sex }\end{array}$ & Severe & 5 & $1.3 \%$ & 3 & $0.8 \%$ \\
\hline $\begin{array}{l}\text { I used threats to make my } \\
\text { partner have sex }\end{array}$ & Severe & 16 & $4.3 \%$ & 2 & $0.5 \%$ \\
\hline
\end{tabular}

The frequency of experiencing different types of violence was determined by counting the number of respondents who experienced at least one of the items in each CTS2 
category - psychological aggression, physical assault and sexual coercion. The following section details the frequency of victimisation and perpetration reported by respondents over the 12 months by each subscale.

Table 6 shows that nearly three-quarters of the sample experienced psychological aggression, indicating that this is a common experience for young adults in dating relationships. Female respondents reported higher rates than male respondents $(74.5 \%$ and $68.3 \%$ respectively) although this was not a significant difference $(\chi 2(1)=.699$, $\mathrm{p}=.403)$. Nearly one-third of the sample (32.6\%) experienced physical assault although there was no significant difference between reported experience by female and male respondents $(33.1 \%$ and $30.0 \%$ respectively; $(\chi 2(1)=.104, \mathrm{p}=.747))$.

The survey revealed relatively high rates of sexual coercion with $32.2 \%$ of female and $25 \%$ of male respondents reporting having experienced sexual coercion. No significant difference was found between female and male respondents in their experience of sexual coercion $(\chi 2(1)=.897, p=.344)$. Of note, in the current study, $8.3 \%$ of respondents reported "forcing their partner to have sex without a condom", or for $15.5 \%$ this was done to them; and $14.7 \%$ of respondents either "insisted on sex with a partner when they did not want to", or for $23.8 \%$ this was done to them.

TABLE 6 THE NUMBER OF RESPONDENTS WHO HAVE EXPERIENCED VIOLENCE BETWEEN AN INTIMATE PARTNER OVER THE PREVIOUS 12 MONTHS BY TYPE OF VIOLENCE

\begin{tabular}{|c|c|c|c|}
\hline \multirow{2}{*}{$\begin{array}{c}\text { Experienced one or more items for the CTS2 } \\
\text { categories }\end{array}$} & Total & \multicolumn{2}{c|}{ Participants } \\
\cline { 3 - 4 } & & Female & Male \\
\hline Respondents experience of violence & \multicolumn{2}{|}{} \\
\hline Psychological aggression & $\mathbf{2 7 5}$ & 234 & 41 \\
& $\mathbf{7 3 . 5 \%}$ & $74.5 \%$ & $68.3 \%$ \\
\hline Physical assault & $\mathbf{1 2 2}$ & 104 & 18 \\
& $\mathbf{3 2 . 6 \%}$ & $33.1 \%$ & $30.0 \%$ \\
\hline Sexual coercion & $\mathbf{1 1 6}$ & 101 & 15 \\
& $\mathbf{3 1 . 0 \%}$ & $32.2 \%$ & $25.0 \%$ \\
\hline Respondents perpetration of violence & \multicolumn{2}{|}{} \\
\hline Psychological aggression & $\mathbf{2 8 4}$ & 238 & 46 \\
\hline Physical assault & $\mathbf{7 5 . 9 \%}$ & $75.8 \%$ & $76.7 \%$ \\
\hline Sexual coercion & $\mathbf{1 4 4}$ & 115 & 29 \\
& $\mathbf{3 8 . 5 \%}$ & $36.6 \%$ & $48.3 \%$ \\
\hline & $\mathbf{8 4}$ & 61 & 23 \\
& $\mathbf{2 2 . 5 \%}$ & $19.4 \%$ & $38.3 \%$ \\
\hline
\end{tabular}


Table 6 presents these findings by type of violence and broken down by gender. It shows that nearly three-quarters of the sample experienced psychological aggression, indicating that this is a common experience for young adults in dating relationships. Female respondents reported higher rates than male respondents $(74.5 \%$ and $68.3 \%$ respectively) although this was not a significant difference $\left(\chi^{2}(1)=.699, \mathrm{p}=.403\right)$. Nearly one-third of the sample (32.6\%) experienced physical assault although there was no significant difference between reported experience by female and male respondents $\left(33.1 \%\right.$ and $30.0 \%$ respectively; $\left.\left(\chi^{2}(1)=.104, p=.747\right)\right)$.

The survey revealed relatively high rates of sexual coercion with $32.2 \%$ of female and $25 \%$ of male respondents reporting having experienced sexual coercion. No significant difference was found between female and male respondents in their experience of sexual coercion $\left(\chi^{2}(1)=.897, p=.344\right)$. Of note, in the current study, $8.3 \%$ of respondents reported "forcing their partner to have sex without a condom", or for $15.5 \%$ this was done to them; and $14.7 \%$ of respondents either "insisted on sex with a partner when they did not want to", or for $23.8 \%$ this was done to them.

These figures align with other studies reporting on data collected from student populations; for example, Harned (2001) reported high rates of psychological abuse with $82 \%$ of women and $87 \%$ of men experiencing this. Physical aggression was experienced by $22 \%$ of women and $21 \%$ of men, and $39 \%$ of women and $30 \%$ of men reported sexual victimisation in dating relationships. These figures suggest that high rates of dating violence are not uncommon when examining the experiences of a, predominantly, student population.

Jackson (1998a) found similarly high rates of emotional abuse with $81.5 \%$ of females and $76.3 \%$ of males experiencing emotional abuse. Her reported rates of sexual coercion were almost twice as high as those in the current study with $76.9 \%$ of females and $67.4 \%$ of males reporting experiencing this type of abuse. However the measure Jackson used was unwanted sexual activity ranging from 'kissing' to 'held down, pushed, punched'. The difference in rates is likely due to her operationalisation of sexual abuse. Jackson's questionnaire was developed directly from the qualitative interviews that she conducted with high school students through a series of focus groups, and did not use an existing validated measure. Questions were focused on 
unwanted sexual activity and items included the following non-consensual sexual activity: kiss, felt up, french kiss, hug, and/or sex.

The present data also shows that sexual coercion is experienced by a large proportion of young adults. This supports findings such as Gavey (1991) who concluded that sexual abuse and coercion is far more commonly experienced by young adults than previous studies have suggested, particularly when questions are included on less severe, but still harmful behaviours such as insisting on sex when their partner did not want to (but did not use physical force), suggesting a significant focus on preventing this type of behaviour is imperative.

Alongside analysis on their role as a victim of dating violence, respondents were also asked about their perpetration of violence towards a dating partner. When respondents in this study reported on this, similar rates to victimisation were found. Of the different types of violence, $75.8 \%$ of female and $76.7 \%$ of male participants perpetrated psychological aggression, $36.6 \%$ of females and $48.3 \%$ of males perpetrated some level of physical assault. Finally, $19.4 \%$ of female and $38.3 \%$ of male respondents reported perpetrating sexual coercion.

These high rates of self-reported perpetration were also found across a number of other studies. For example, Shorey, Brasfield, Zapor, Febres and Stuart's (2015) study of 204 college males found that $54.9 \%$ reported perpetration of psychological aggression, $18.9 \%$ perpetrated physical assault and nearly a quarter of men reported perpetrating sexual aggression $(24.3 \%)$.

Significance tests were calculated on this study data using chi-square analysis. For reported perpetration of violence, men and women reported perpetrating psychological aggression and physical assault at the same rates, however male respondents were significantly more likely to report perpetrating sexual coercion compared to females' reports of their perpetration $\left(\chi^{2}(1)=9.282, p=.002\right)$. In other words, gender symmetrical violence was seen for perpetration of psychological aggression and physical assault but not for sexual coercion.

These figures align with studies such as Harned (2001) whose analysis on a sample of 874 undergraduate and graduate students from a USA university found that, while men 
and women reported similar rates of overall perpetration, men were in fact more likely to report higher levels of perpetration of emotional abuse and sexual violence.

\section{OVERLAP OF TYPES OF VIOLENCE}

An important element in understanding the use and experience of violence is whether certain tactics are used in isolation or if multiple tactics are used in a violent relationship. As reported by Cale et al., (2016) physical violence rarely occurs in isolation from other types of violence. Studies such as O'Leary and Maiuro (2001) show that physical violence is often present with psychological violence.

To explore this in the current study, two Venn diagrams were developed (see Figure 1) that show the number of respondents who reported the range of combinations possible in their experience of violence - as a victim of their partner's violence in the previous 12 months (left hand diagram) and as a perpetrator of violence against their partner in the previous 12 months (right hand diagram). The diagrams do not include the 82 respondents who reported that they were not a victim of any form of violence in the previous 12 months and the 73 respondents who did not report perpetrating any violence against their partner in the previous 12 months.

FIGURE 1 EXPERIENCE OF ONE OR MORE TYPES OF VIOLENCE FOR VICTIMS AND PERPETRATORS OF DATING VIOLENCE IN THE PREVIOUS 12 MONTHS

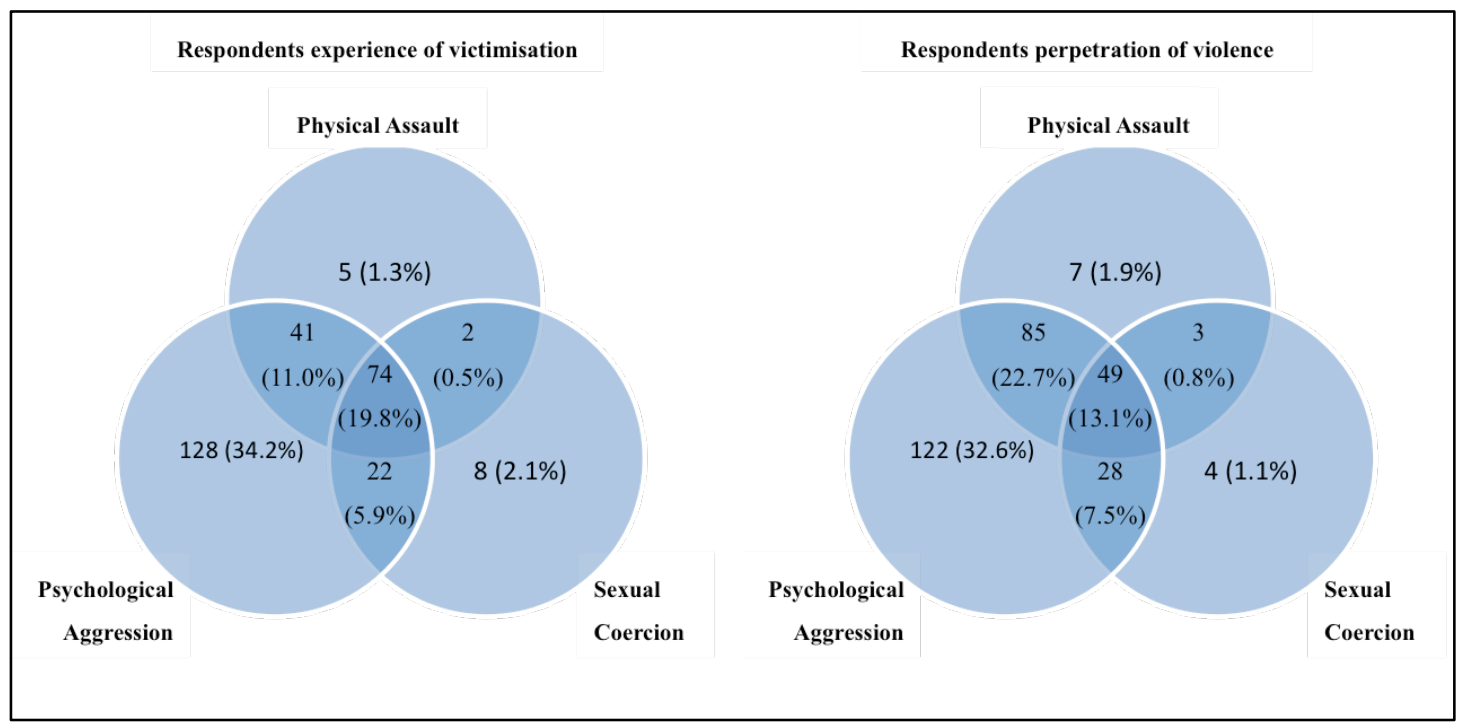


Figure 1 demonstrates that psychological aggression is the most commonly experienced form of violence, on its own and in combination with other types of violence. Physical assault was more likely to occur in the context of experiencing other forms of violence, for both those experiencing violence from their partner and those perpetrating violence towards their partner. Likewise, sexual coercion was less likely to be experienced on its own, but was experienced in combination with psychological aggression only or in combination with psychological aggression and physical assault.

\section{Mutual Violence}

As the previous section demonstrates, dating violence is a common experience as both a victim and perpetrator. With such a significant proportion of young adults reporting this, it appears inevitable that within dating relationships an individual will be both a victim and perpetrator of dating violence. In this current study analysis was conducted to look at the reciprocity of violence by respondents and their partner and found that, where violence is reported, it was much more likely to be experienced in a reciprocal pattern with participants reporting that they and their partner were both violent, at some level, in the relationship.

Figure 2 shows that 279 respondents reported both they and their partner were violent in the relationship (74.6\%) with only 65 respondents reporting that both partners were non-violent (17.4\%). There were 30 participants who reported that only one partner was violent and the other partner was not $(8.0 \%)$ with just $3 \%$ of relationships where the respondent was a victim-only and 5\% where they were a perpetrator-only.

Chi-square analysis was conducted to test the association between respondents being violent or not, compared to their partner being violent or not. Results show significant differences suggesting that if the respondent was violent, their partner was also likely to be violent, and conversely if the participant was not violent their partner was also likely to be non-violent $\left(\chi^{2}(1)=213.316, p=.000\right)$. 

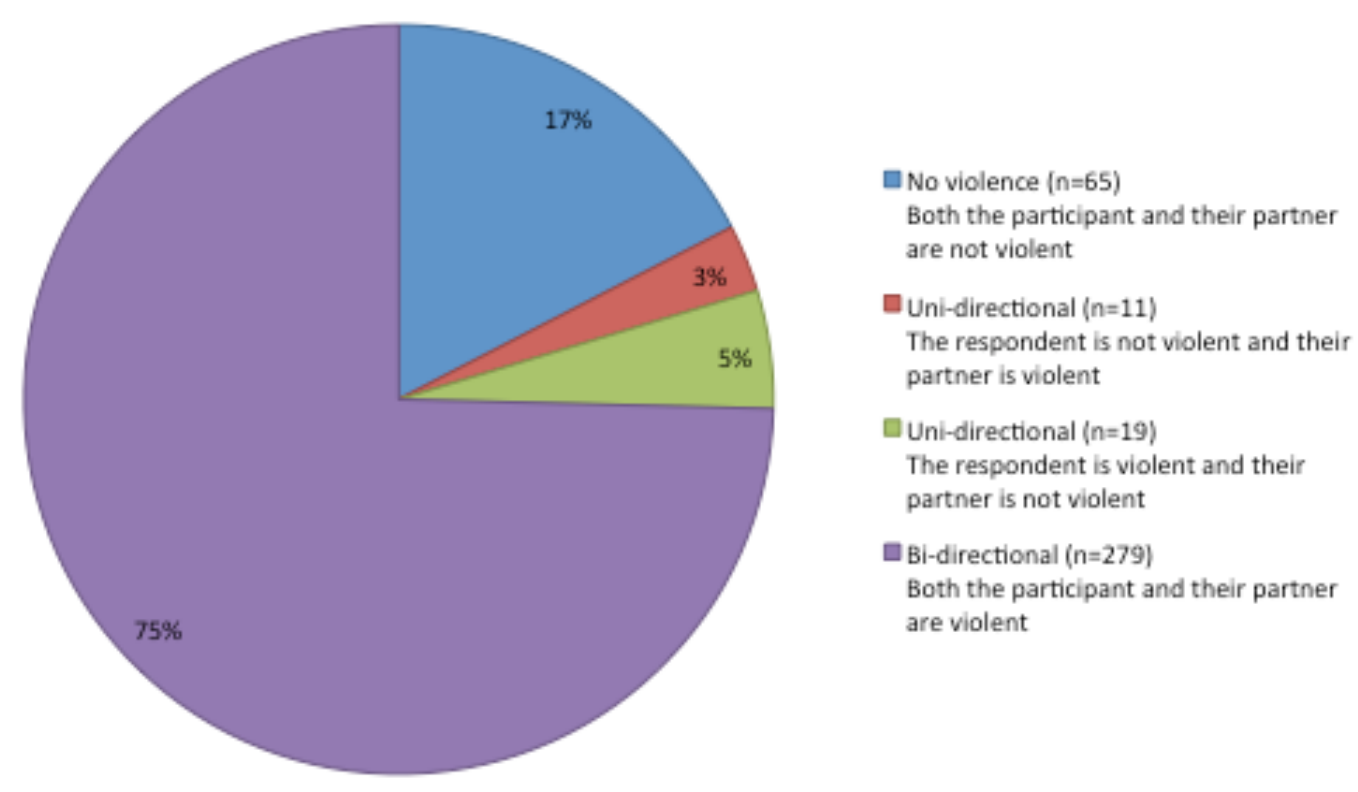

This pattern has been reported in a number of other studies including by Chiodo, et al. (2012); Rapoza and Baker (2008); and Straus (2004). These studies also showed that in relationships where there was a bi-directional pattern of violence, the violence was more frequent and more severe when compared to relationships where there is unilateral violence (Hautala, Sittner Hartshorn, Armenta, \& Whitbeck, 2017).

\section{Minor AND SEVERE VIOLENCE}

Within the CTS2 scale Straus et al. (1996) classify some items as minor events and others as severe events. Table 7 shows that across all types of violence minor forms of violence were more commonly experienced than severe forms of violence. Table 5 supports this in that the most extreme forms of violence such as "I used a knife or gun on my partner"; "I burned or scalded my partner on purpose"; "I used force to make my partner have oral or anal sex" were reported less than $1 \%$ of the time".

Table 7 shows the reported use and experience of each type of violence broken down by minor and severe ratings. Table 7 shows that across all types of violence minor forms of violence were more commonly experienced than severe forms of violence. 
Table 5 supports this in that the most extreme forms of violence such as "I used a knife or gun on my partner"; "I burned or scalded my partner on purpose"; "I used force to make my partner have oral or anal sex" were reported less than $1 \%$ of the time".

TABLE 7 RESPONDENTS' EXPERIENCE OF MINOR AND SEVERE VIOLENCE BETWEEN AN INTIMATE PARTNER OVER THE PREVIOUS 12 MONTH

\begin{tabular}{|c|c|c|c|c|c|c|}
\hline \multirow{2}{*}{$\begin{array}{c}\text { EXPERIENCE Minor } \\
\text { and severe acts of } \\
\text { violence }\end{array}$} & \multicolumn{3}{|c|}{$\begin{array}{c}\text { Respondents victimisation } \\
\text { from violence }\end{array}$} & \multicolumn{3}{|c|}{$\begin{array}{c}\text { Respondents perpetration } \\
\text { of violence }\end{array}$} \\
\hline & Total & Females & Males & Total & Females & Males \\
\hline \multicolumn{7}{|l|}{ Psychological aggression } \\
\hline Minor only & $\begin{array}{c}176 \\
64.0 \%\end{array}$ & $\begin{array}{c}148 \\
63.2 \%\end{array}$ & $\begin{array}{c}28 \\
68.3 \%\end{array}$ & $\begin{array}{c}203 \\
71.5 \%\end{array}$ & $\begin{array}{c}171 \\
84.2 \%\end{array}$ & $\begin{array}{c}32 \\
69.6 \%\end{array}$ \\
\hline Severe & $\begin{array}{c}99 \\
36.0 \% \\
\end{array}$ & $\begin{array}{c}86 \\
36.8 \% \\
\end{array}$ & $\begin{array}{c}13 \\
31.7 \% \\
\end{array}$ & $\begin{array}{c}81 \\
28.5 \%\end{array}$ & $\begin{array}{c}67 \\
28.2 \% \\
\end{array}$ & $\begin{array}{c}14 \\
30.4 \% \\
\end{array}$ \\
\hline \multicolumn{7}{|l|}{ Physical assault } \\
\hline Minor only & $\begin{array}{c}68 \\
55.7 \%\end{array}$ & $\begin{array}{c}56 \\
53.8 \%\end{array}$ & $\begin{array}{c}12 \\
66.7 \%\end{array}$ & $\begin{array}{c}95 \\
66.0 \%\end{array}$ & $\begin{array}{c}81 \\
70.4 \%\end{array}$ & $\begin{array}{c}14 \\
48.3 \%\end{array}$ \\
\hline Severe & $\begin{array}{c}54 \\
44.3 \% \\
\end{array}$ & $\begin{array}{c}48 \\
46.2 \% \\
\end{array}$ & $\begin{array}{c}6 \\
33.3 \\
\end{array}$ & $\begin{array}{c}49 \\
34.0 \% \\
\end{array}$ & $\begin{array}{c}34 \\
29.6 \% \\
\end{array}$ & $\begin{array}{c}15 \\
51.7 \% \\
\end{array}$ \\
\hline \multicolumn{7}{|l|}{ Sexual coercion } \\
\hline Minor only & $\begin{array}{c}94 \\
81.0 \%\end{array}$ & $\begin{array}{c}81 \\
80.2 \%\end{array}$ & $\begin{array}{c}13 \\
86.7 \%\end{array}$ & $\begin{array}{c}80 \\
95.2 \%\end{array}$ & $\begin{array}{c}60 \\
98.4 \%\end{array}$ & $\begin{array}{c}20 \\
87.0 \%\end{array}$ \\
\hline Severe & $\begin{array}{c}22 \\
19.0 \%\end{array}$ & $\begin{array}{c}20 \\
19.8 \%\end{array}$ & $\begin{array}{c}2 \\
13.3 \%\end{array}$ & $\begin{array}{c}4 \\
4.8 \%\end{array}$ & $\begin{array}{c}1 \\
1.6 \%\end{array}$ & $\begin{array}{c}3 \\
13.0 \%\end{array}$ \\
\hline
\end{tabular}

Across respondent's victimisation, minor violence was more likely to be reported, which is consistent with Magdol et al's (1997) analysis of the Dunedin Longitudinal study where they reported similar patterns of IPV seriousness. Similarly, Rapoza and Baker (2008) reported from their study of dating couples that most of the physical assault was minor assault with only a small number of severe assaults reported across their sample of 171 young people.

Looking across the types of violence, male respondents were more likely to report being victims of minor violence compared to female respondents. Conversely, female respondents were more likely to report experiencing severe violence compared to male 
respondents. However these differences were not significant across either level of violence or by type of violence.

There were however significant differences between male and female respondents when looking at reported perpetration of severe violence. Across all types of violence females were more likely to report perpetrating minor violence at higher rates than male participants, while male participants were more likely to report perpetrating higher rates of severe violence. While these differences were not significant for psychological violence, they were for the use of severe physical violence with male respondents being significantly more likely to report perpetrating severe physical violence compared to female participants $(p=.006)^{5}$. Likewise, male respondents were also significantly more likely to report perpetrating severe sexual coercion compared to female participants $(p=.014)$.

As shown in Figure 3 those coded as experiencing severe violence across the different types of violence were also likely to report experiencing minor violence but for the purpose of the above analysis they were classified in the severe category only. This means that participants are only counted once allowing for the totals to add up to the overall proportions of dating violence reported in Table 4.

\footnotetext{
${ }^{5}$ The Fisher Exact test was used to test significance as the frequency assumptions for Pearson's chi-square did not hold.
} 


\section{FIGURE 3 CROSSOVER OF MINOR AND SEVERE VIOLENCE EXPERIENCED BETWEEN AND INTIMATE}

\section{PARTNER OVER THE PREVIOUS 12 MONTHS}
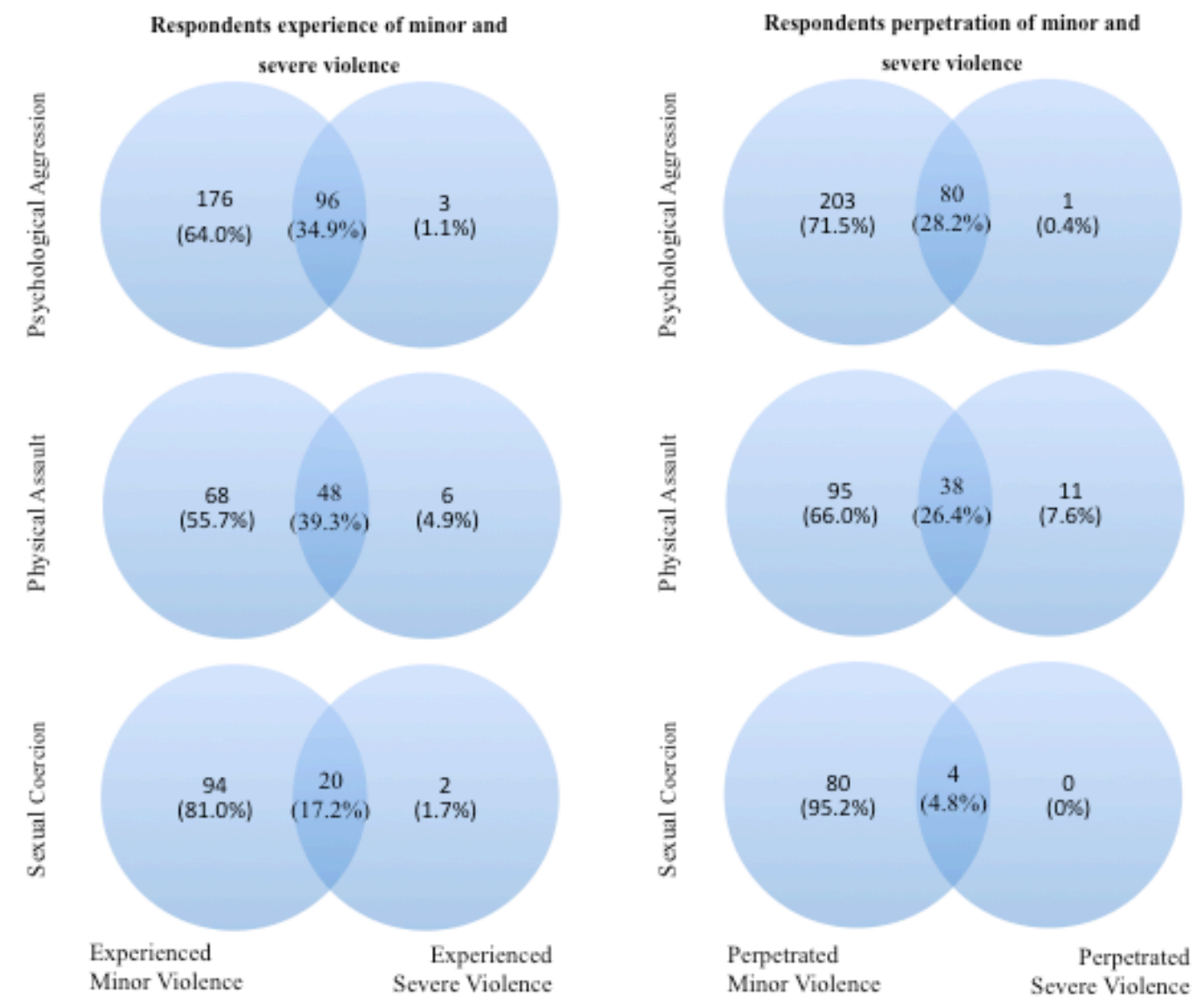

This section has added further evidence to the significant body of dating literature from the USA that shows that a large majority of young people in dating relationships experience some form of violence, and that these young people are often experiencing multiple forms of violence, not just one type in isolation. The absence of violence was only found in around $20 \%$ of relationships, demonstrating the far reach of violence and the need to for attention on this issue as one of significant concern.

These findings also demonstrate the dyadic nature of dating violence with a large proportion of young adults describing their dual role as both a perpetrator and a victim of violence, highlighting the complexity in how we describe and intervene in this type of experience.

At first glance these findings also indicate support for the notion that women and men experience some forms of dating violence at similar rates, and as both perpetrators and victims. However further analysis looking at the proportion of men and women who 
experienced different types of violence showed a more pronounced gendered picture. These findings support a number of other studies that demonstrate that research into violence in dating and other intimate relationships needs to provide context and investigate the impact when looking into the experiences of violence, requiring researchers to collect a range of information to understand the complexities that underlie these dynamic and harmful relationships.

\section{SECTION THREE: INJURY FROM DATING VIOLENCE}

As discussed in Chapter Two, the discussion around the equivalence of women's violence makes it imperative to provide context and investigate the impact of violence in our analysis and narrative of dating violence. There is a need to include information about the nature and motives of the violence being experienced by young adults to test whether male and female perpetrated violence have the same impact on victims (Hamberger, 2005).

One way to develop a deeper understanding of the nature of such violence is to look at the levels of injury experienced by respondents, both in their role as a victim and/or perpetrator. Investigating the level of reported injury is an important step as a significant body of research provides general support for the argument that male violence has far more negative impacts than does female violence in intimate relationships when looking at severe physical injury, medical treatment, psychological outcomes and fear (Holtzworth-Munroe, 2005; Harned, 2001).

The injury scale from the CTS2 (Straus et al., 1996) was used in this study and included six items that described types of physical injury that participants could report experiencing (see Table 9 for the list of these six items).

\section{EXPERIENCING INJURY AS A RESULT OF DATING VIOLENCE}

Table 8 summarises the frequency of physical injury experienced in the previous 12 months for both victims and perpetrators. Twelve percent of respondents reported receiving a physical injury as a result of their partner's violence over the past 12 months. Female respondents reported experiencing higher rates of physical injury than male respondents over the previous 12 months ( $13.1 \%$ and $6.7 \%$ respectively), although this was not a significant difference $(p=.116)$. Overall $8.3 \%$ of respondents 
reported causing physical injury to their dating partner in the previous 12 months (5.4\% females; $23.3 \%$ males). Significant differences were found between male and female perpetration of injury with male participants perpetrating significantly higher levels of injury during the 12-month period $(p=.000){ }^{6}$

TABLE 8 THE NUMBER OF RESPONDENTS WHO EXPERIENCED INJURY BY AN INTIMATE PARTNER OVER THE PREVIOUS 12 MONTHS

\begin{tabular}{|l|c|c|c|}
\hline Experienced one or more Injury items for & \multirow{2}{*}{$\begin{array}{c}\text { Total } \\
\text { the CTS2 categories }\end{array}$} & \multicolumn{2}{|c|}{ Participants } \\
\cline { 3 - 4 } & & Female & Male \\
\hline Injury experienced by participants & $\mathbf{4 5}$ & 41 & 4 \\
& $\mathbf{1 2 . 0 \%}$ & $13.1 \%$ & $6.7 \%$ \\
\hline Injury caused by the participants & $\mathbf{3 1}$ & 17 & 14 \\
& $\mathbf{8 . 3 \%}$ & $5.4 \%$ & $23.3 \%$ \\
\hline
\end{tabular}

These rates are consistent with studies such as Archer's (2000). In his meta-analysis, he found that women perpetrated physical acts of violence in their dating relationship at a higher rate than males, however he also found that when looking at the impact of the violence women were significantly more likely to sustain injury inflicted by their male dating partner, with $62 \%$ of those injured being women.

Table 9 shows the distribution of injury items that respondents reported experiencing or using to cause injury, by gender, in the previous 12 months. The most commonly reported items were experiencing "a sprain, bruise, or small cut" and "feeling physical pain that still hurt the next day". Those items least likely to be experienced were "needing to see a doctor because of a fight" and "experiencing a broken bone from a fight with my partner".

\footnotetext{
${ }^{6}$ The Fisher Exact test was used to test significance, as the frequency assumptions for Pearson's chi-square did not hold.
} 
TABLE 9 THE DISTRIBUTION OF RESPONSE TO EACH INJURY ITEM FROM THE CTS2 IN THE PREVIOUS 12 MONTHS

\begin{tabular}{|l|l|c|c|c|c|}
\hline Injury item from the CTS2 & & \multicolumn{2}{|c|}{$\begin{array}{c}\text { Injury experienced } \\
\text { by participants }\end{array}$} & \multicolumn{2}{|c|}{$\begin{array}{c}\text { Injury caused by } \\
\text { the participants }\end{array}$} \\
\hline & & Frequency & $\%$ & Frequency & $\%$ \\
\hline $\begin{array}{l}\text { I had a sprain, bruise, or small cut } \\
\text { because of a fight with my partner }\end{array}$ & Minor & 30 & $8.0 \%$ & 20 & $5.3 \%$ \\
\hline $\begin{array}{l}\text { I felt physical pain that still hurt the } \\
\text { next day because of a fight with my } \\
\text { partner }\end{array}$ & Minor & 34 & $9.1 \%$ & 15 & $4.0 \%$ \\
\hline $\begin{array}{l}\text { I passed out from being hit on the } \\
\text { head by my partner in a fight }\end{array}$ & Severe & 4 & $1.1 \%$ & 12 & $3.2 \%$ \\
\hline $\begin{array}{l}\text { I went to a doctor because of a fight } \\
\text { with my partner }\end{array}$ & Severe & 9 & $2.4 \%$ & 20 & $5.3 \%$ \\
\hline $\begin{array}{l}\text { I needed to see a doctor because of a } \\
\text { fight with my partner, but I didn't }\end{array}$ & Severe & 7 & $1.9 \%$ & 20 & $5.3 \%$ \\
\hline $\begin{array}{l}\text { I had a broken bone from a fight with } \\
\text { my partner }\end{array}$ & Severe & 3 & $0.8 \%$ & 3 & $0.8 \%$ \\
\hline
\end{tabular}

Table 10 shows the number of tactics respondents reported experiencing or using to cause physical injury, by gender, in the previous 12 months. The CTS 2 provides six different items that respondents reported against.

TABLE 10 THE NUMBER OF ITEMS RESPONDENTS EXPERIENCED AS A VICTIM AND PERPETRATOR OF INJURY IN THE PREVIOUS 12 MONTHS

\begin{tabular}{|l|c|c|c|c|c|c|}
\hline & \multicolumn{3}{|c|}{ Injury experienced by participants } & \multicolumn{2}{c|}{ Injury caused by the participants } \\
\hline $\begin{array}{c}\text { Number } \\
\text { of injury } \\
\text { items } \\
\text { from the } \\
\text { CTS2 }\end{array}$ & Total & Female & Male & Total & Female & Male \\
respondent & respondent & & respondent & respondent \\
\hline $\mathbf{0}$ items & $\mathbf{3 2 9}$ & 273 & 56 & $\mathbf{3 4 3}$ & 297 & 46 \\
& $\mathbf{8 8 . 0 \%}$ & $86.9 \%$ & $93.3 \%$ & $\mathbf{9 1 . 7 \%}$ & $94.6 \%$ & $76.7 \%$ \\
\hline $\mathbf{1}$ item & $\mathbf{1 0}$ & 9 & 1 & $\mathbf{1 3}$ & 10 & 3 \\
& $\mathbf{2 . 7 \%}$ & $2.9 \%$ & $1.7 \%$ & $\mathbf{\%}$ & $4.1 \%$ & $5.0 \%$ \\
\hline $\mathbf{2}$ items & $\mathbf{5}$ & 5 & 0 & $\mathbf{7}$ & 5 & 2 \\
& $\mathbf{1 . 3 \%}$ & $1.6 \%$ & $0.0 \%$ & $\mathbf{1 . 9 \%}$ & $1.6 \%$ & $3.3 \%$ \\
\hline $\mathbf{3}$ items & $\mathbf{1}$ & 1 & 0 & $\mathbf{4}$ & 1 & 3 \\
& $\mathbf{0 . 3 \%}$ & $0.3 \%$ & $0.0 \%$ & $\mathbf{1 . 1 \%}$ & $0.3 \%$ & $5.0 \%$ \\
\hline $\mathbf{4}$ items & $\mathbf{5}$ & 4 & 1 & $\mathbf{4}$ & 0 & 4 \\
& $\mathbf{1 . 3 \%}$ & $1.3 \%$ & $1.7 \%$ & $\mathbf{1 . 1 \%}$ & $0.0 \%$ & $6.7 \%$ \\
\hline $\mathbf{5}$ items & $\mathbf{6}$ & 4 & 2 & $\mathbf{1}$ & 0 & 1 \\
& $\mathbf{1 . 6 \%}$ & $1.3 \%$ & $3.3 \%$ & $\mathbf{0 . 3} \%$ & $0.0 \%$ & $1.7 \%$ \\
\hline $\mathbf{6}$ items & $\mathbf{1 8}$ & 18 & 0 & $\mathbf{2}$ & 1 & 1 \\
& $\mathbf{4 . 8 \%}$ & $5.7 \%$ & $0.0 \%$ & $\mathbf{0 . 5} \%$ & $0.3 \%$ & $1.7 \%$ \\
\hline
\end{tabular}


Of the 45 respondents who experienced physical injury caused by their partner in the previous 12 months, just under half reported that their partner used four or fewer injury items $(n=21,47 \%)$, however just over half of respondents reported that their partner used five or six injury items to cause physical injury ( $n=24,53 \%)$. Of the 31 respondents who reported causing physical injury towards their partner, most reported using four or fewer injury items $(n=28,90 \%)$ with only three respondents reporting the use of five or more injury items.

\section{MINOR AND SEVERE INJURY EXPERIENCED}

Within the gender symmetry debate and the discussion on equivalence of violence between men and women, there is debate over whether women have the ability to cause similar levels of physical injury compared to men (Hamberger, 2005). The analysis above on the proportion of young men and women who experienced injury and who caused injury shows that there is a gendered element, with young men being more likely to cause injury and young women being more likely to be harmed by their male partner.

Table 11 shows that there was a strong gender difference in reporting of physical injury. Young men were significantly more likely than young women to report causing minor injury, with $21.7 \%$ of the 60 male respondents reporting they caused minor injury compared to $5.4 \%$ of the 314 female respondents $(p=.000)$. Likewise, young men were significantly more likely to report causing severe injury with $15 \%$ of the 60 young male respondents reporting they caused serious injury compared to $5.4 \%$ of the 314 female respondents $(p=.000)$. Female participants also reported higher rates of being injured by their dating partner compared to male participants, although this was not significant for either the experience of minor or severe violence.

The counts against the acts that are presented in Table 11 were combined into a dichotomous variable where those who experienced severe injury were classified first with the remaining participants counted as experiencing minor injury only. As shown in Figure 4 those coded as experiencing severe injury were likely to also report experiencing minor injury but for the purpose of the above analysis, they were classified in the severe category only. This means that participants are only counted once allowing for the totals to add up to the overall proportions of dating violence reported in Table 8 . 
TABLE 11 RESPONDENTS' EXPERIENCE AS A VICTIM AND PERPETRATOR OF MINOR AND SEVERE INJURY OVER A 12-MONTH PERIOD

\begin{tabular}{|c|c|c|c|c|c|c|}
\hline & \multicolumn{2}{|c|}{$\begin{array}{c}\text { Injury experienced by } \\
\text { participants }\end{array}$} & \multicolumn{3}{c|}{ Injury caused by } \\
& \multicolumn{3}{|c|}{ participants } \\
\hline Experienced minor and & Total & Females & Males & Total & Females & Males \\
severe injury* & & & & & & \\
\hline Minor & & & & & & 13 \\
& $\mathbf{4 3}$ & 39 & 4 & $\mathbf{3 0}$ & 17 & $13 \%$ \\
\hline Severe & $\mathbf{1 1 . 5 \%}$ & $12.4 \%$ & $6.7 \%$ & $\mathbf{8 . 0} \%$ & $5.4 \%$ & $21.7 \%$ \\
& $\mathbf{2 6}$ & 25 & 1 & $\mathbf{1 1}$ & 2 & 9 \\
& $\mathbf{7 . 0} \%$ & $8.0 \%$ & $1.7 \%$ & $\mathbf{2 . 9 \%}$ & $0.6 \%$ & $15.0 \%$ \\
\hline
\end{tabular}

* Note that participants could be counted as reporting both minor and severe injury, therefore the total count of injury in this table is larger than the total number of participants who reported experiencing injury in Table 8.

Looking at this overlap in Figure 4, of the 45 young adults who experienced injury, 19 experienced minor injury only and two experienced severe injury only, while 24 respondents experienced both minor and severe injury in combination.

\section{FIGURE 4 CROSSOVER OF MINOR AND SEVERE INJURY EXPERIENCED BY AN INTIMATE PARTNER OVER} THE PREVIOUS 12 MONTHS

Respondents experience of minor and severe injury

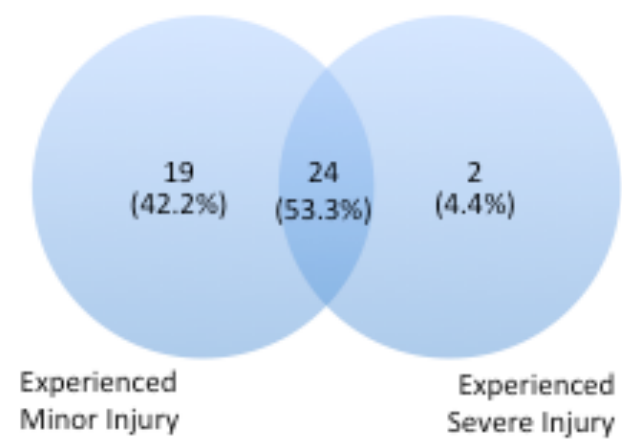

Respondents perpetration of minor and severe injury

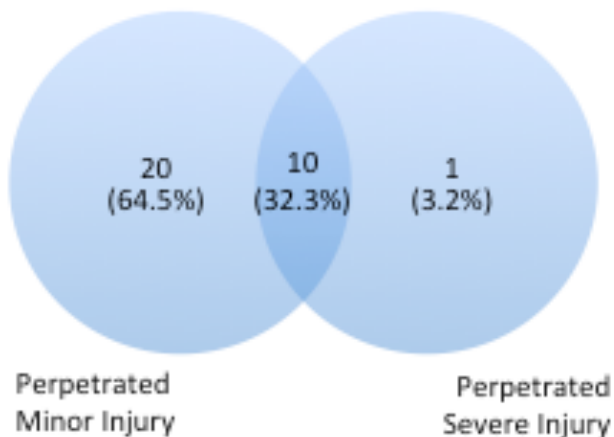

A slightly different pattern was found for the self-reported perpetration of injury with more young adults reporting causing minor injury-only. Of the 31 young adults who reported causing injury to a dating partner, only one reported causing severe injury to their partner with 20 reporting minor injury only and 10 young adults reporting that they caused both minor and severe injury at some point during the dating relationship. 


\section{CHAPTER SUMMARY}

This chapter has provided data from one of the few New Zealand-based samples of young adults that reports on violence in the context of contemporary New Zealand dating relationships. This study adds to the body of dating violence research that shows that young adults are experiencing high rates of violence in dating relationships. While most of this violence was more minor in nature, a number of these young adults experienced a range of more severe forms of violence which suggests that a range of experiences are encountered by young dating adults, particularly those from a largely student sample.

A number of chi-square tests were run to look at the relationship between gender and violence victimisation and perpetration over 12 months. While overall levels of experiencing and perpetrating any form of violence in the previous 12 months was reported at similar rates between the young men and women in this study, once the different types of violence were more closely analysed, the severity of that violence and the impact from that violence revealed a strong gendered pattern.

While not statistically significant, young women were more likely to be victims of psychological aggression, physical assault and sexual coercion; conversely young men were significantly more likely to report perpetrating physical assault and sexual coercion. Young men were significantly more likely to perpetrate severe physical violence compared to young female respondents, and this was also the case for perpetration of both minor and severe sexual coercion. This gendered pattern was also evident for those experiencing injury, although the numbers were smaller so caution should be exercised.

The findings from this chapter demonstrate the complex nature of measuring and understanding the experience of violence in dating relationships. Like other intimate relationship studies, such as Capaldi and Owen's (2001) USA-based study and Robertson and Murachver's (2007b) New Zealand-based study, women reported their own use of violence, and likewise men reported their victimisation at the hands of their female partner. However, the findings in this study do support placing dating violence, and violence in other intimate relationships, in the context of a gendered analysis. 
The debate as to the nature and motives of this violence and the need to develop more nuanced frameworks for understanding the complex nature of such relational violence warrant further investigation. One approach is to use the typology of violence developed by Johnson (2008), which include a range of data from different populations, to account for the different experiences of violence and to develop a more sophisticated explanation of the nature and experience of violence (Enander, 2011).

In the next chapter I will apply Johnson's typology by replicating his methodological protocol to this online survey data to re-create his typology categories and then examine the distribution of the types of violence that occurred in dating relationships in this study. This will form the foundation for later chapters that look to assess the validity and utility of the typology as a framework to understand the different experiences of violence in dating relationships and their complexity. 


\title{
CHAPTER SIX - REPLICATING JOHNSON'S TYPOLOGY
}

\author{
Although we appear to know a good deal about what was initially a most \\ surprising incidence of partner violence in dating relationships, this literature \\ is as plagued by lack of distinctions as is the marital violence literature. \\ (Johnson \& Ferraro, 2000, p. 951)
}

The previous chapter showed the unsurprising results that violence is experienced by a large proportion of young adults in the context of dating relationships and that for those who experience the more severe forms of violence there is a higher chance of being injured, particularly young women by their male dating partner.

In this chapter I apply Johnson's (2008) typological framework and his corresponding analysis protocol to create his typology categories and then examine the distribution of the types of violence that occur in dating relationships. Participants were categorised as non-violent, intimate terrorism or situational couple violence using the categories described in Chapter Three. A series of significance test were run using chi-square and Fisher Exact tests. An alpha level of .05 was used as the significance criterion for these tests.

\section{SECTION ONE: LEVELS OF COERCIVE CONTROL}

As discussed in Chapter One coercive control is a central concept in the literature about violence against women where it is theorised as being a central feature of men's use of violence in intimate relationships in order to maintain power and control (Pence \& Paymar, 1993). The presence of coercive control in a relationship can be so effective in gaining control and compliance it is argued that the use of physical violence is not always necessary; therefore a large proportion of coercive control happens in the absence of physical and/or sexual violence (Stark \& Hester, 2019).

It is this notion of the presence of coercive control that saw Johnson (2008) use it as the distinguishing element when describing different types of IPV. As detailed in Chapter Three, Johnson argues that the presence of coercive control helps explain the 
conflicting gendered results across studies (Meier, 2015). The nature of high coercive control makes for more terrifying and impactful violence.

To replicate Johnson's typology the Coercive Control Scale was used (Johnson et al., 2008). The Coercive Control Scale is the critical component, alongside the CTS2 responses, used to develop Johnson's typology. It measures respondents' own controlling behaviour and the controlling behaviour of their dating partner and consists of nine items. Table 12 shows the items used to measure this construct and the reported frequency across these items.

From these results there is a wide range of controlling behaviours reportedly used by both the participant and by their partner. Table 12 shows that the most commonly described item of control was being jealous or possessive $(38.5 \%)$, this was reported at similar rates for both male (45.0\%) and female respondents $(37.3 \%)(p=.311)$. Males were significantly more likely to report that they frighten their partner compared to female respondents $-20.0 \%$ and $1.6 \%$ respectively $(p=.000)$. Male respondents were also significantly more likely to make their female partners feel inadequate $(p=.002)$; and to prevent them from knowing about or having access to the family income $(p=.000)$.

Similarly, the most commonly reported control tactic used by respondents' partners was being jealous or possessive (27.5\%). Respondents reported that $17.9 \%$ of partners made them feel inadequate, compared to only $6.4 \%$ reporting that they did this to their partner. Male partners were also significantly more likely to frighten their partner compared to female respondents in this study $(p=.048)$. 
TABle 12 The distribution Of ReSPONSES TO EACH OF THE COERCIVE CONTROL SCALE ITEMS OVER THE PREVIOUS 12 MONTHS

\begin{tabular}{|c|c|c|c|}
\hline & \multicolumn{3}{|c|}{ Respondents Coercive Control } \\
\hline & Total & $\begin{array}{c}\text { Female } \\
\text { respondent }\end{array}$ & $\begin{array}{c}\text { Male } \\
\text { respondent }\end{array}$ \\
\hline I am jealous or possessive & $\begin{array}{c}144 \\
38.5 \%\end{array}$ & $\begin{array}{c}117 \\
37.3 \%\end{array}$ & $\begin{array}{c}27 \\
45.0 \%\end{array}$ \\
\hline I try to provoke arguments & $\begin{array}{c}75 \\
20.1 \% \\
\end{array}$ & $\begin{array}{c}58 \\
18.5 \% \\
\end{array}$ & $\begin{array}{c}17 \\
28.3 \% \\
\end{array}$ \\
\hline $\begin{array}{l}\text { I try to limit their contact with family and } \\
\text { friends }\end{array}$ & $\begin{array}{c}16 \\
4.3 \% \\
\end{array}$ & $\begin{array}{c}8 \\
2.5 \% \\
\end{array}$ & $\begin{array}{c}8 \\
13.3 \% \\
\end{array}$ \\
\hline $\begin{array}{l}\text { I insist on knowing who they are with at } \\
\text { all times }\end{array}$ & $\begin{array}{c}61 \\
16.3 \% \\
\end{array}$ & $\begin{array}{c}48 \\
15.3 \% \\
\end{array}$ & $\begin{array}{c}13 \\
21.7 \% \\
\end{array}$ \\
\hline $\begin{array}{l}\text { I call them names or put them down in } \\
\text { front of others }\end{array}$ & $\begin{array}{c}30 \\
8.0 \%\end{array}$ & $\begin{array}{c}21 \\
6.7 \%\end{array}$ & $\begin{array}{c}9 \\
15.0 \%\end{array}$ \\
\hline I make them feel inadequate & $\begin{array}{c}24 \\
6.4 \%\end{array}$ & $\begin{array}{c}14 \\
4.5 \% \\
\end{array}$ & $\begin{array}{c}10 \\
16.7 \% \\
\end{array}$ \\
\hline I shout or swear at them & $\begin{array}{c}71 \\
19.0 \% \\
\end{array}$ & $\begin{array}{c}60 \\
19.1 \% \\
\end{array}$ & $\begin{array}{c}11 \\
18.3 \% \\
\end{array}$ \\
\hline I frighten them & $\begin{array}{c}17 \\
4.5 \% \\
\end{array}$ & $\begin{array}{l}5 \\
1.6 \% \\
\end{array}$ & $\begin{array}{c}12 \\
20.0 \% \\
\end{array}$ \\
\hline \multirow[t]{3}{*}{$\begin{array}{l}\text { I prevent them from knowing about or } \\
\text { having access to the family income }\end{array}$} & $\begin{array}{c}14 \\
3.7 \% \\
\end{array}$ & $\begin{array}{c}1 \\
0.3 \% \\
\end{array}$ & $\begin{array}{c}13 \\
21.7 \% \\
\end{array}$ \\
\hline & \multicolumn{3}{|c|}{ Partners Coercive Control } \\
\hline & Total & $\begin{array}{l}\text { Female } \\
\text { partner }\end{array}$ & Male partner \\
\hline $\mathrm{He} / \mathrm{she}$ is jealous or possessive & $\begin{array}{c}103 \\
27.5 \% \\
\end{array}$ & $\begin{array}{c}19 \\
26.8 \% \\
\end{array}$ & $\begin{array}{c}84 \\
27.7 \% \\
\end{array}$ \\
\hline $\mathrm{He} / \mathrm{she}$ tries to provoke arguments & $\begin{array}{c}75 \\
20.1 \% \\
\end{array}$ & $\begin{array}{c}14 \\
19.7 \% \\
\end{array}$ & $\begin{array}{c}61 \\
20.1 \% \\
\end{array}$ \\
\hline $\begin{array}{l}\text { He/she tries to limit my contact with } \\
\text { family and friends }\end{array}$ & $\begin{array}{c}39 \\
10.4 \%\end{array}$ & $\begin{array}{c}3 \\
4.2 \%\end{array}$ & $\begin{array}{c}36 \\
11.9 \%\end{array}$ \\
\hline $\begin{array}{l}\text { He/she insist on knowing who I am with } \\
\text { at all times }\end{array}$ & $\begin{array}{c}69 \\
18.4 \%\end{array}$ & $\begin{array}{c}11 \\
15.5 \%\end{array}$ & $\begin{array}{c}58 \\
19.1 \%\end{array}$ \\
\hline $\begin{array}{l}\text { He/she calls me names or puts me down } \\
\text { in front of others }\end{array}$ & $\begin{array}{c}44 \\
11.8 \% \\
\end{array}$ & $\begin{array}{c}6 \\
8.5 \% \\
\end{array}$ & $\begin{array}{c}38 \\
12.5 \% \\
\end{array}$ \\
\hline $\mathrm{He} / \mathrm{she}$ makes me feel inadequate & $\begin{array}{c}67 \\
17.9 \% \\
\end{array}$ & $\begin{array}{c}8 \\
11.3 \% \\
\end{array}$ & $\begin{array}{c}59 \\
19.5 \% \\
\end{array}$ \\
\hline $\mathrm{He} / \mathrm{she}$ shouts or swear at me & $\begin{array}{c}53 \\
14.2 \% \\
\end{array}$ & $\begin{array}{c}14 \\
19.7 \% \\
\end{array}$ & $\begin{array}{c}39 \\
12.9 \% \\
\end{array}$ \\
\hline $\mathrm{He} /$ she frightens me & $\begin{array}{c}29 \\
7.8 \% \\
\end{array}$ & $\begin{array}{c}1 \\
1.4 \% \\
\end{array}$ & $\begin{array}{c}28 \\
9.2 \% \\
\end{array}$ \\
\hline $\begin{array}{l}\text { He/she prevents me from knowing about } \\
\text { or having access to the family income }\end{array}$ & $\begin{array}{c}17 \\
4.5 \%\end{array}$ & $\begin{array}{c}1 \\
1.4 \%\end{array}$ & $\begin{array}{c}16 \\
5.3 \%\end{array}$ \\
\hline
\end{tabular}


Table 13 shows the distribution of items that participants reported using to control their partner and the number of controlling tactics that their partner used to control them. The numbers across some categories are too small to run significance testing, however a pattern is strongly evident whereby males are reported to use a greater number of controlling acts towards their partner compared to female respondents. Similarly, male partners also reported using a greater number of controlling tactics towards the respondents compared to female partners. These patterns are discussed in the next section when they are combined to determine the level of coercive control used (see Table 14).

TABLE 13 THE NUMBER OF ITEMS OF COERCIVE CONTROL USED BY RESPONDENTS AND THEIR PARTNER IN THE PREVIOUS 12 MONTHS

\begin{tabular}{|c|c|c|c|c|c|c|}
\hline \multirow[b]{2}{*}{$\begin{array}{l}\text { Number of } \\
\text { items used }\end{array}$} & \multicolumn{3}{|c|}{ Respondent's Coercive Control } & \multicolumn{3}{|c|}{ Partner's Coercive Control } \\
\hline & Total & $\begin{array}{c}\text { Female } \\
\text { respondent }\end{array}$ & $\begin{array}{c}\text { Male } \\
\text { responde } \\
\text { nt }\end{array}$ & Total & $\begin{array}{l}\text { Female } \\
\text { partner }\end{array}$ & Male partner \\
\hline 0 items & $\begin{array}{c}165 \\
44.1 \%\end{array}$ & $\begin{array}{c}146 \\
46.5 \%\end{array}$ & $\begin{array}{c}19 \\
31.7 \% \\
\end{array}$ & $\begin{array}{c}199 \\
53.2 \% \\
\end{array}$ & $\begin{array}{c}40 \\
56.3 \% \\
\end{array}$ & $\begin{array}{c}159 \\
52.5 \% \\
\end{array}$ \\
\hline 1 item & $\begin{array}{c}91 \\
24.3 \%\end{array}$ & $\begin{array}{c}77 \\
24.5 \%\end{array}$ & $\begin{array}{c}14 \\
23.3 \%\end{array}$ & $\begin{array}{c}60 \\
16.0 \%\end{array}$ & $\begin{array}{c}11 \\
15.5 \%\end{array}$ & $\begin{array}{c}49 \\
16.2 \%\end{array}$ \\
\hline 2 items & $\begin{array}{c}50 \\
13.4 \%\end{array}$ & $\begin{array}{c}43 \\
13.7 \% \\
\end{array}$ & $\begin{array}{c}7 \\
11.7 \% \\
\end{array}$ & $\begin{array}{c}36 \\
9.6 \% \\
\end{array}$ & $\begin{array}{c}7 \\
9.9 \% \\
\end{array}$ & $\begin{array}{c}29 \\
9.6 \% \\
\end{array}$ \\
\hline 3 items & $\begin{array}{c}35 \\
9.4 \%\end{array}$ & $\begin{array}{c}31 \\
9.9 \%\end{array}$ & $\begin{array}{c}4 \\
6.7 \%\end{array}$ & $\begin{array}{c}29 \\
7.8 \%\end{array}$ & $\begin{array}{c}7 \\
9.9 \% \\
\end{array}$ & $\begin{array}{c}22 \\
7.3 \%\end{array}$ \\
\hline 4 items & $\begin{array}{c}18 \\
4.8 \%\end{array}$ & $\begin{array}{c}15 \\
4.8 \%\end{array}$ & $\begin{array}{c}3 \\
5.0 \%\end{array}$ & $\begin{array}{c}11 \\
2.9 \%\end{array}$ & $\begin{array}{c}4 \\
5.6 \%\end{array}$ & $\begin{array}{c}7 \\
2.3 \%\end{array}$ \\
\hline 5 items & $\begin{array}{c}5 \\
1.3 \%\end{array}$ & $\begin{array}{c}2 \\
0.6 \%\end{array}$ & $\begin{array}{c}3 \\
5.0 \%\end{array}$ & $\begin{array}{c}13 \\
3.5 \%\end{array}$ & $\begin{array}{c}1 \\
1.4 \%\end{array}$ & $\begin{array}{c}12 \\
4.0 \%\end{array}$ \\
\hline 6 items & $\begin{array}{c}4 \\
1.1 \%\end{array}$ & $\begin{array}{c}0 \\
0.0 \%\end{array}$ & $\begin{array}{c}4 \\
6.7 \%\end{array}$ & $\begin{array}{c}14 \\
3.7 \%\end{array}$ & $\begin{array}{c}1 \\
1.4 \%\end{array}$ & $\begin{array}{c}13 \\
4.3 \%\end{array}$ \\
\hline 7 items & $\begin{array}{c}4 \\
1.1 \%\end{array}$ & $\begin{array}{c}0 \\
0.0 \%\end{array}$ & $\begin{array}{c}4 \\
6.7 \%\end{array}$ & $\begin{array}{c}3 \\
0.8 \%\end{array}$ & $\begin{array}{c}0 \\
0.0 \%\end{array}$ & $\begin{array}{c}3 \\
1.0 \%\end{array}$ \\
\hline 8 items & $\begin{array}{c}1 \\
0.3 \%\end{array}$ & $\begin{array}{c}0 \\
0.0 \%\end{array}$ & $\begin{array}{c}1 \\
1.7 \%\end{array}$ & $\begin{array}{c}1 \\
0.3 \%\end{array}$ & $\begin{array}{c}0 \\
0.0 \%\end{array}$ & $\begin{array}{c}1 \\
0.3 \%\end{array}$ \\
\hline 9 items & $\begin{array}{c}1 \\
0.3 \%\end{array}$ & $\begin{array}{c}0 \\
0.0 \%\end{array}$ & $\begin{array}{c}1 \\
1.7 \%\end{array}$ & $\begin{array}{c}8 \\
2.1 \%\end{array}$ & $\begin{array}{c}0 \\
0.0 \%\end{array}$ & $\begin{array}{c}8 \\
2.6 \%\end{array}$ \\
\hline
\end{tabular}

\section{ClassificAtion OF High AND LOW COERCIVE CONTROL}

To develop Johnson's typology, the first step is to sort participants and their partners into no, low or high control groups. To determine the level of control used by respondents and their partners Johnson's recommended cut-off was used, whereby those who reported experiencing no acts were classified as experiencing no coercive 
control, those who reported fewer than five acts were considered low control and those who reported experiencing five or more coercive controlling acts were classified as high control.

Table 14 shows the distribution of the participants and their partner into the control categories. These results show that $36.4 \%$ of respondents reported that their partner used low levels of coercive control while $10.4 \%$ of respondents reported experiencing high levels of coercive control at the hands of their dating partner. Reporting on their own use of control, $51.9 \%$ of respondents were classified as low control while $4.0 \%$ were classified as high coercive control.

TABLE 14 THE DISTRIBUTION OF PARTICIPANTS AND THEIR PARTNER INTO LEVEL OF CONTROL USED IN THE PREVIOUS 12 MONTHS

\begin{tabular}{|c|c|c|c|}
\hline & \multirow[t]{2}{*}{ Total } & \multicolumn{2}{|c|}{ Gender of partner } \\
\hline & & Female & Male \\
\hline \multicolumn{4}{|c|}{ Partner's use of Coercive Control } \\
\hline No Coercive Control & $\begin{array}{c}199 \\
53.2 \%\end{array}$ & $\begin{array}{c}40 \\
56.3 \%\end{array}$ & $\begin{array}{c}159 \\
52.5 \%\end{array}$ \\
\hline Low Coercive Control & $\begin{array}{c}136 \\
36.4 \% \\
\end{array}$ & $\begin{array}{c}29 \\
40.8 \% \\
\end{array}$ & $\begin{array}{c}107 \\
35.3 \% \\
\end{array}$ \\
\hline \multirow[t]{3}{*}{ High Coercive Control } & $\begin{array}{c}39 \\
10.4 \%\end{array}$ & $\begin{array}{c}2 \\
2.8 \%\end{array}$ & $\begin{array}{c}37 \\
12.2 \%\end{array}$ \\
\hline & Total & \multicolumn{2}{|c|}{ Gender of respondent } \\
\hline & & Female & Male \\
\hline \multicolumn{4}{|c|}{ Respondent's use of Coercive Control } \\
\hline No Coercive Control & $\begin{array}{c}165 \\
44.1 \% \\
\end{array}$ & $\begin{array}{c}146 \\
46.5 \% \\
\end{array}$ & $\begin{array}{c}19 \\
31.7 \% \\
\end{array}$ \\
\hline Low Coercive Control & $\begin{array}{c}194 \\
51.9 \% \\
\end{array}$ & $\begin{array}{c}166 \\
52.9 \% \\
\end{array}$ & $\begin{array}{c}28 \\
46.7 \% \\
\end{array}$ \\
\hline High Coercive Control & $\begin{array}{c}15 \\
4.0 \%\end{array}$ & $\begin{array}{c}2 \\
0.6 \%\end{array}$ & $\begin{array}{c}13 \\
21.7 \%\end{array}$ \\
\hline
\end{tabular}

Chi-square analysis was performed and revealed that levels of partners' use of coercive control (respondents experiencing violence) varied by gender with male partners more likely to be classified in the high coercive control category compared to female partners, $12.2 \%$ and $2.8 \%$ respectively.

Gender is also strongly associated with the level of coercive control that respondents reported they used in relationship. A significant difference was found for respondents' use of coercive control as a function of their gender, showing that male respondents were significantly more likely to report using higher levels of coercive control than 
female respondents, while female respondents were significantly more likely to report using low or no coercive control $\left(\chi^{2}(2)=58.431, \mathrm{p}=.000\right)$.

\section{SECTION TwO: CREATION OF THE TYPOLOGY}

Following the classification of respondents and their partners into low, high or no coercive control categories, respondents were coded into Johnson's typology following his procedure (Personal Communications, 12 November 2014). Figure 5 shows the decision tree used to classify respondents and their partners into Johnson's typology. First, respondents and their partners were split by those who have perpetrated physical assault and then by the level of coercive control they enacted. From this, they were classified into one of three categories, situational couple violence (SCV), intimate terrorism (IT) or non-violent (NV).

Of the total sample of 374 respondents, 144 reported that they had perpetrated physical violence $(38.5 \%)$. Of these:

- $\quad 9.0 \%(\mathrm{n}=13)$ were classified as High Coercive Control - therefore labelled as intimate terrorist

- $70.8 \%(\mathrm{n}=102)$ were classified as Low Coercive Control and were labelled as situational couple violence, and

- $20.1 \%(n=29)$ participants who reported using violence but did not report using any coercive control were also classified as situational couple violence.

In total, of the 144 respondents who reported being physically violent, $9.0 \%$ were classified as intimate terrorist and $91.0 \%$ classified as situational couple violence. The 230 respondents that did not perpetrate physical violence were all categorised as nonviolent. 


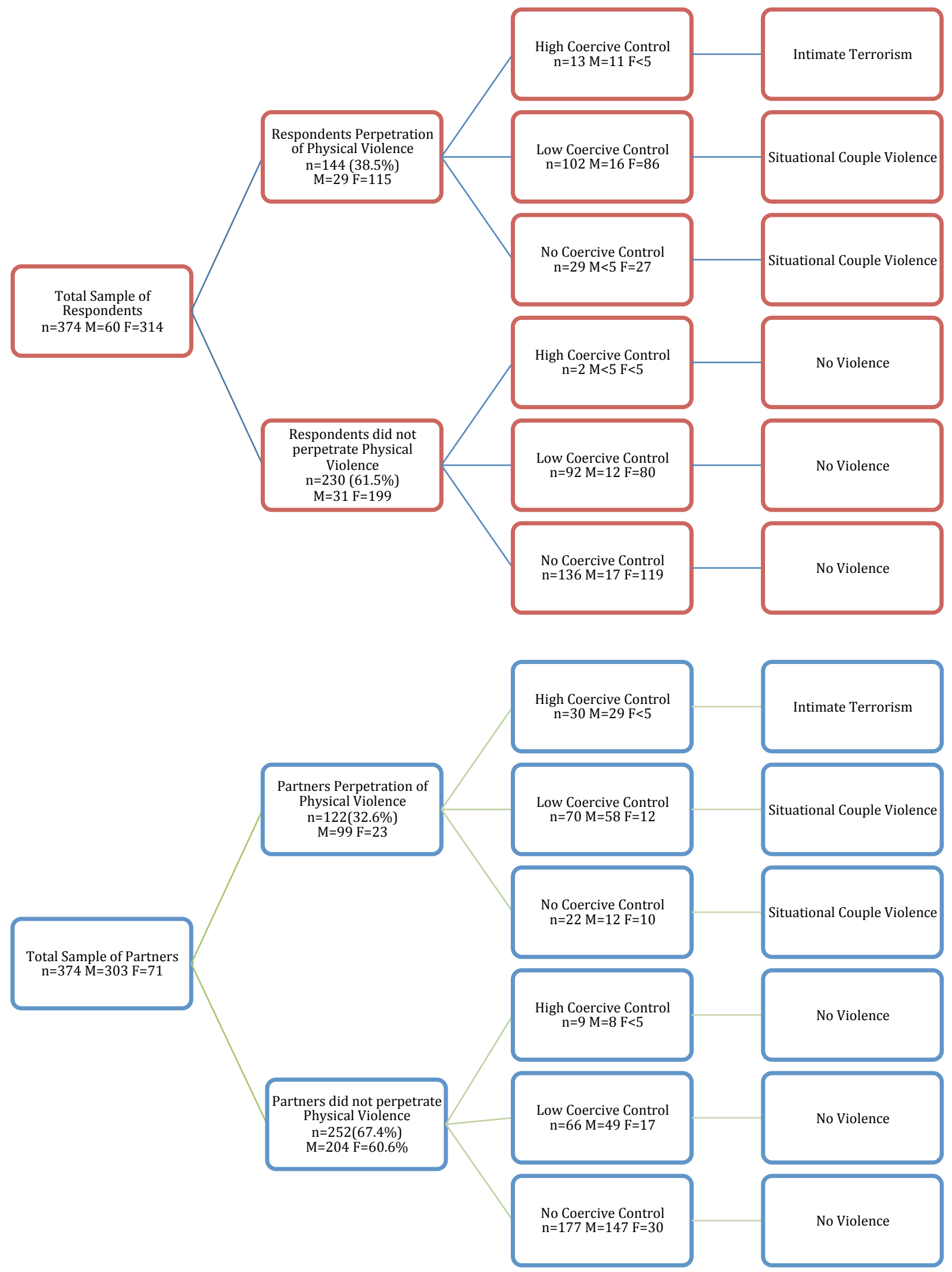


The same procedure was applied to respondents' partners. Of the 374 dating partners, 122 were reported as having perpetrated physical violence against respondents $(32.6 \%)$. Of these:

- $122,24.6 \%(\mathrm{n}=30)$ were classified as High Coercive Control - therefore labelled as intimate terrorist

- $57.4 \%(\mathrm{n}=70)$ were classified as Low Coercive Control and were labelled as situational couple violence, and

- $18.0 \%(\mathrm{n}=22)$ participants who reported not using any coercive control were also classified as situational couple violence.

\section{SECTION THREE: DEMOGRAPHIC AND RELATIONSHIP CHARACTERISTICS}

Table 15 shows the distribution of the typology categories by the range of demographic variables that were collected about the respondent and the nature of their dating relationship.

Of all these demographics, the only significant finding was by gender, where female participants were significantly more likely to report perpetrating levels of violence and control classified as situational couple violence compared to male participants $(p=.001)$. Conversely, female respondents were less likely to report levels of violence and control that are classified as intimate terrorism compared to male respondents.

However, the analysis also showed that some female respondents did commit physical violence in combination with high coercive controlling behaviours. While these are extremely small numbers, results do show that women can commit violence and exhibit coercive control that is consistent with this classification. The results align with other studies, particularly largely community samples and student samples. For example, in a British-study Graham-Kevan and Archer (2003) analysed women living in shelters and their partners $(n=86)$, men in domestic violence treatment programmes and their partners $(n=8)$, male prisoners and their partners $(n=192)$ and a sample of male and female students $(n=208)$. They reported that females were more commonly classified as situational couple violence while men were nearly six times more likely than the female participants to be classified as intimate terrorist. Likewise, Bogaerts et al's (2011) nationwide study of participants in the Netherlands found that men were 
more likely than women to be categorised as intimate terrorist $(21 \%$ and $14 \%$ respectively). In contrast, Straus and Gozjolko's (2014) international dating violence study found men and women were equally likely to be categorised as intimate terrorist.

TABLE 15 CHARACTERISTICS OF THE RESPONDENTS AND RELATIONSHIP DESCRIPTIONS BY TYPOLOGY CLASSIFICATION

\begin{tabular}{|c|c|c|c|c|c|c|}
\hline & \multicolumn{2}{|c|}{$\begin{array}{l}\text { Intimate } \\
\text { Terrorist }\end{array}$} & \multicolumn{2}{|c|}{$\begin{array}{c}\text { Situational } \\
\text { Couple Violence } \\
\end{array}$} & \multicolumn{2}{|c|}{ Non-violent } \\
\hline & $\mathbf{N}$ & $\%$ & N & $\%$ & $\mathbf{N}$ & $\%$ \\
\hline \multicolumn{7}{|l|}{ Gender of respondent } \\
\hline Female & 5 & $1.6 \%$ & 110 & $35.0 \%$ & 199 & $63.4 \%$ \\
\hline Male & 8 & $13.3 \%$ & 21 & $35.0 \%$ & 31 & $51.7 \%$ \\
\hline \multicolumn{7}{|l|}{ Age of respondent } \\
\hline $18-21$ years & 10 & $3.8 \%$ & 99 & $37.2 \%$ & 157 & $59.0 \%$ \\
\hline $22-25$ years & 3 & $2.8 \%$ & 32 & $29.6 \%$ & 73 & $67.6 \%$ \\
\hline \multicolumn{7}{|l|}{ Current or ex-partner } \\
\hline Current partner & 11 & $3.6 \%$ & 111 & $36.8 \%$ & 180 & $59.6 \%$ \\
\hline Ex-partner & 2 & $2.8 \%$ & 20 & $27.8 \%$ & 50 & $69.4 \%$ \\
\hline \multicolumn{7}{|l|}{ Sexual orientation } \\
\hline Heterosexual & 13 & $3.8 \%$ & 122 & $35.3 \%$ & 211 & $61.0 \%$ \\
\hline Same-sex & 0 & $0.0 \%$ & 9 & $32.1 \%$ & 19 & $67.9 \%$ \\
\hline \multicolumn{7}{|l|}{ Living situation } \\
\hline $\begin{array}{l}\text { Live in the family home }- \text { not } \\
\text { with partner }\end{array}$ & 1 & $2.5 \%$ & 18 & $45.0 \%$ & 21 & $52.5 \%$ \\
\hline $\begin{array}{l}\text { Live with flatmates - not with } \\
\text { partner }\end{array}$ & 8 & $3.6 \%$ & 71 & $31.7 \%$ & 145 & $64.7 \%$ \\
\hline Living with partner & 4 & $3.6 \%$ & 42 & $38.2 \%$ & 64 & $58.2 \%$ \\
\hline \multicolumn{7}{|l|}{ Length of relationship } \\
\hline $3-11$ months & 4 & $2.4 \%$ & 48 & $28.6 \%$ & 116 & $69.0 \%$ \\
\hline $12-23$ months & 2 & $2.3 \%$ & 38 & $43.7 \%$ & 47 & $54.0 \%$ \\
\hline $24-47$ months & 5 & $5.2 \%$ & 39 & $40.2 \%$ & 53 & $54.6 \%$ \\
\hline 4 years or more & 2 & $9.1 \%$ & 6 & $27.3 \%$ & 14 & $63.6 \%$ \\
\hline \multicolumn{7}{|l|}{ How found out about survey } \\
\hline Agency & 3 & $6.3 \%$ & 17 & $35.4 \%$ & 28 & $58.3 \%$ \\
\hline In class & 4 & $2.2 \%$ & 70 & $38.0 \%$ & 110 & $59.8 \%$ \\
\hline Social media / Word of mouth & 6 & $4.2 \%$ & 44 & $31.0 \%$ & 92 & $64.8 \%$ \\
\hline
\end{tabular}


Across the remaining variables no significant differences were found, likely due to the small number of participants characterised as intimate terrorist. Of note though was the distribution of the typology categories when looking at the length of the dating relationships, and how the participant found out about the survey. Table 15 revealed that participants who were classified as intimate terrorists had been in a relationship for a greater length of time than those participants classified as situational couple violence.

Table 15 also shows that participants in the intimate terrorist category were also more likely to have been recruited through an agency sample (6.3\%), with $4.2 \%$ finding out about the survey through word of mouth. Those participants who found out about the survey in class were least likely to be classified as intimate terrorist $(2.2 \%)$ and more likely to be classified as situational couple violence (38.0\%). While not statistically significant, it is supported by findings from Hines and Douglas (2010), and by Johnson, Leone and $\mathrm{Xu}$ (2014) that it is more likely that situational couple violence is found in general survey samples while intimate terrorism is more likely to be found in agency or court samples.

The data also to a large extent confirmed my reasoning that intimate terrorism and violent resistance would not appear in survey data, because intimate terrorists would not want to implicate themselves and their victims would fear the consequences should their intimate terrorist partner learn that they had been answering questions about their family life. (Johnson, 2017, p. 153)

\section{DYADIC FOUR CATEGORY TYPOLOGY CLASSIFICATION}

The benefit of collecting information on both partners is the resulting ability to combine both the respondent and their partner's use of violence and control into dyadic typological categories. Using the classification of the respondent and their partner, a combined four-cluster classification was developed accounting for the dyadic nature of the violent and controlling behaviour. Where both the respondent and their partner were reported as non-violent, their relationship typology classification was also 'non-violent'. Figure 6 depicts how the four categories were developed and the associated numbers calculated from this study. Essentially, if an individual (respondent or partner) is violent, he or she is classified as intimate terrorist (IT) if he/she is high control and his/her partner is either non-violent or violent but low 
control; violent resistance (VR) is assigned if he/she is low control and his/her partner is violent and high control; situational couple violence (SCV) applies if he/she is low control and his/her partner is either non-violent or violent and low control; and, mutual violent control (MVC) is used if he/she and his/her partner are both violent and high control.

These figures are presented to provide insight into the dyadic nature of dating violence, however no further analyses were run as there was little likelihood of finding any significant results.

There were three relationships from this sample that were classified as mutual violence, i.e., where both participants were classified as intimate terrorist. There were also 26 couples where there was violent resistance, i.e., where one partner was classified as intimate terrorist and the other as situational couple violence. So even in a sample that is over-represented by students, there are still cases of more extreme dating violence, as classified through Johnson's (2008) typology.

\section{FIGURE 6 THE DYADIC INTERACTION OF THE INDIVIDUAL CATEGORIES TO CREATE THE FOUR}

\section{CATEGORY TYPOLOGY}

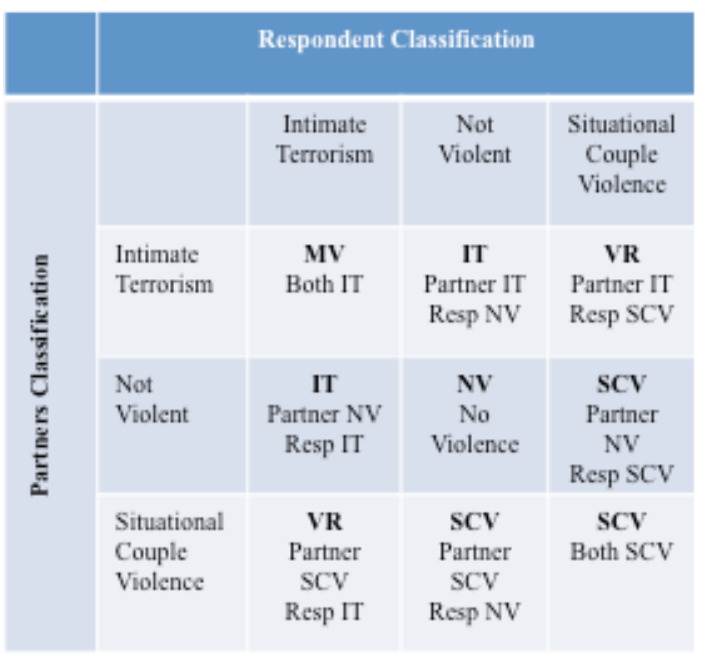

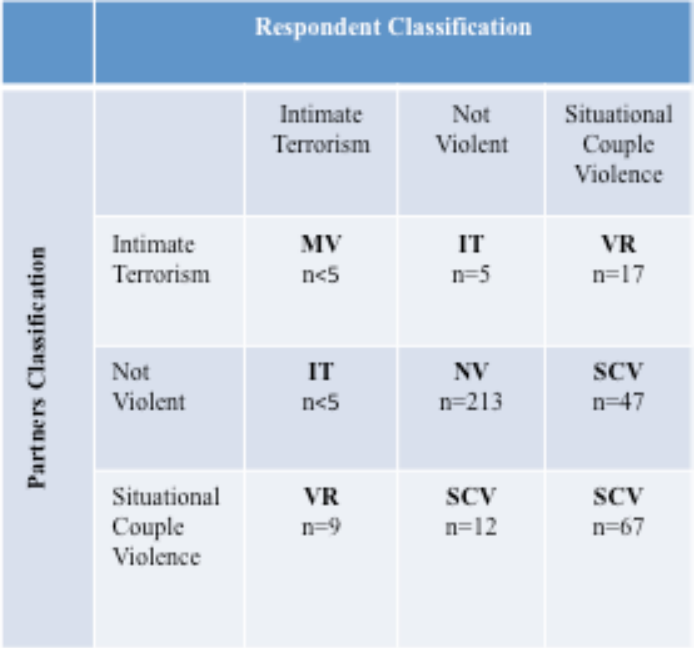

It is likely that the distribution of relationships falling across the four categories is a result of the deliberate sampling of both students and crisis support clients, and also likely that those classified, as intimate terrorist and mutual violent relationships were participants recruited through crisis support agencies as they provided a targeted sample where more severe violence had occurred. This is supported by findings in Table 15 looking at the two-way individual classification which found that those 
classified as being in intimate terrorism relationships were more likely to be from an agency sample, while those in the situation couple violence category were more likely to be from the student sample (note the limitations in the variable about how participants found out about this survey, detailed in Chapter Four).

\section{CHAPTER SUMMARY}

In this chapter I applied Johnson's (2008) typological framework and his corresponding analysis protocol to re-create his typology categories and then examine the distribution of the types of violence that occurred in dating relationships. Chapter Five demonstrated that there is a complex picture underpinning young adult dating relationships with cases of both young men and young women reporting victimisation in such a relationships and/or perpetrating violence.

Johnson's typology was easily applied, as the current analysis supported his typological categories, although there were small numbers of participants in the intimate terrorist and mutual violent control categories. This is consistent with studies that show less intimate terrorism-based violence occurring in population-based samples (Hines \& Douglas, 2010). The findings reported here support the importance and value of applying some form of clustering of victim/perpetrator characteristics to better understand the different aetiology of violence that lies within.

The question that naturally arises asks whether Johnson's typology is just a statistical manipulation of a cohort or does it strongly represent the experience of those that are classified, in this case young adults in dating relationships? In the next chapter, Chapter Seven, I will therefore explore the narratives of young adults who described the current social and cultural context for dating in New Zealand. In Chapters Eight and Nine I will report these narratives in the context of Johnson's typology labels. This qualitative analysis will look more deeply into whether Johnson's typology makes sense when used to frame what young adults say about their experiences in dating relationships. 


\title{
ChAPTER SEVEN - CURRENT SOCIAL AND CULTURAL CONTEXT OF DATING
}

\author{
To me, dating is what smart women (and men) do... New Zealand seems to \\ treat singledom in a very odd way. Not only is it a failure, it's a situation to be \\ avoided at all costs because, socially, it's not the done thing. (McDonald, \\ 2009)
}

In this chapter I will explore young adults' narratives to capture what dating means for them and how this experience was constructed within the current New Zealand context. In the previous chapter Johnson's (2008) procedure was applied to the survey data and participants and their dating partners were categorised into his typology categories: situational couple violence and intimate terrorist.

Before testing the thesis research question to see whether young adults' narratives of dating violence support Johnson's statistically-derived typology, it is important to understand the current social context that my sample of young adults is navigating. As described in Chapter One, the social context within which young adults currently live is a very different landscape from that of just a few decades ago (McHugh \& Frieze, 2006).

Contemporary young adults navigate their own development, identities and relationships in a social environment that is much more fluid with a less clear path to successful attainment of adulthood, including potential relationship formation. These contextual issues may affect the degree to which Johnson's typology is applicable to current young adults' experiences. This social context will also provide a backdrop for the final chapter and the discussion on ways to support and educate young adults to maintain healthy romantic relationships and reduce the experience of violent dating relationships.

This chapter provides a narrative from interviews with 16 young adults who were recruited through the online survey (see Chapter Four for a detailed description of the method, respondents and analysis protocol). The chapter is divided into two sections. The first section discusses findings on participants' narratives of the current dating context in New Zealand including narratives on socialising; forming romantic 
relationships; social norms; and the attitudes, values and beliefs that influence the nature and experience of dating for young adults in New Zealand. The second section focuses on the influence and proliferation of technology, social media and popular culture in young people's everyday lives, the impact of wider social influences, and how these together have affected young adult attitudes, behaviour and beliefs supportive of violence and control.

To protect anonymity, participants have been given pseudonyms. The associated typology category is the actual category assigned through the quantitative analysis in Chapter Six. See Appendix 8 for a description of interview participants and their partners' typology classification.

\section{SECTION ONE: THE CURRENT DATING CONTEXT IN NEW ZEALAND}

A central element that ran across all the participants' narratives was their interaction with alcohol. Over the past decades there has been increasing attention in the Western world, including New Zealand, on the "cultures of intoxication". Hutton et al. (2013) describe a dominant "culture of intoxication" in New Zealand, where this term represents the "well-developed national culture of drinking to intoxication" (Hutton, Griffin, Lyons, Niland, \& McCreanor, 2016, p. 73). This theme was supported through participant narratives, where they described going out to party with their friends. This pattern of socialising often occurred in public places, and included drinking heavily and engaging in sexual encounters with people they may have only just met.

When participants described this culture of intoxication they used terms such as going out to "get wasted", "get plastered" or "get smashed", demonstrating their intention to drink to excess. This culture of intoxication has been supported by a relaxation of alcohol laws, lowering the age of legal purchase of alcohol and an expansion of public places for young people to seek both leisure and pleasure (James, 2010). These factors have supported the increase in young adults planning to go out and get excessively drunk or 'wasted' in what Hutton et al. (2013, p. 454) term as "determined drunkenness" and the growing "night-time economy" (participation in the consumer cultures of clubs, pubs, and bars). This excessive drinking and acceptability of casual 
sexual encounters or 'hooking-up' reflected a normalised cultural expectation where it is atypical for young adults to not participate in such behaviours (Hutton et al., 2013).

Yeah we go out drinking all the time, it's just what you do. Usually we preload at a mate's first, that's the cheap way to get smashed. Then we head into town to find chicks. The best night out is student night at the pub, it's a big night, you get totally smashed. It's just what you do you with your crew, that's how we hang out. It gives us awesome stories to laugh about after. (Regan, IT) We party it up all the time, you gotta let loose and relax. We go out and meet up with friends, you have to always go 'cause you definitely don't want to miss out on an epic night. You don't want to be the one who wasn't there for some epic night of mayhem. (Jack, SCV)

Student nights are big, you go out and get as drunk as you can. I just want to feel out of control, ya know, just relax and be crazy without any worries. It's a time to be with my girlfriends and have a good time. That's how we create memories. (Jessica, IT)

What participants showed in these quotes is that engaging in the night-time economy and this culture of intoxication is part of their identity as young people and is part of how they form bonds and relationships with their peers and intimate partners. A similar narrative was found in a study by Hutton et al. (2013) where a largely student population discussed the normality of binge drinking and their engagement in the night-time economy. This was also described by Fry (2011) where socialising and drinking were part of how young adults conformed to social norms and formed bonds and relationships:

Being drunk signified socialisation, enjoyment, fun and pleasure, with anticipated excitement felt prior to the consumption event and 'war stories' discussed post-consumption. Socialising in the night-time economy was a euphemism for 'having a good time'. In turn, 'having a good time' was a euphemism for drinking to excess. (Fry, 2011, pp. 67)

Participants' narratives on their engagement in the night-time economy and drinking practices were consistent for both male and female participants. What seemed 
apparent from participants was the traditionally male culture of drinking and socialising had shifted to one where it was now socially acceptable for females to drink in the same setting. Hutton (2012) also found, in a study of New Zealand university students, that their engagement in going out partying and to partake in binge-drinking practices was not gendered, and that there were similar patterns of drinking for both young men and young women. The observation of the lack of gendered engagement in this night-time economy was described by Day, Gough and McFadden (2004, p. 172):

Here, the implication appears to be that it makes no sense to talk about laddism or ladettes anymore because alcohol consumption and other traditionally 'male' or 'masculine' endeavours are no longer the preserve of men. Indeed, an overall discursive pattern which was identified during analysis was one which constructs drinking culture as becoming increasingly feminised ... the "feminisation of drinking".

However, some evidence of strong gendered rules and norms were identified in relation to how young women should behave and certain expectations on them that were not placed on young men. Similarly, Hutton et al. (2016) posit that there is an expectation that young women engage in the night-time economy while maintaining a level of respectability. This means navigating a fine line between having fun but having to maintain a level of "decency, desirability and respectability", behaving appropriately, so that they do not become unfeminine and undesirable.

They must at all times avoid the spectre of the "drunken slag", while trying to attain the "right" image and the "right" level of intoxication. (Hutton et al., 2016, p. 86)

This section has highlighted the dominant drinking culture and almost universal engagement by participants, who were predominantly university students, in the night-time economy that supported a culture of going out, partying and getting drunk. This section has also demonstrated that participants viewed this culture as a largely normative way to interact and socialise with their peers. 


\section{HOOKING-UP - ROMANTIC ENCOUNTERS}

Within the strong drinking culture and engagement in the night-time economy participants described an implicit link to that culture and to casual sex, hook-ups and the establishment of relationships. Nearly all participants (14 out of 16) described how they went 'out into town' (socialising with friends in public bars, getting drunk) with the goal of 'hooking-up' with someone.

\section{CASUAL SEX EXPERIENCE}

The term 'hook-up' was used frequently by participants and described scenarios where sexual intimacy would result; casual sexual encounters included a range of scenarios which are covered in Beres and Farvid's (2010, p. 381) definition in their study of heterosexual casual sex experiences in Canada and New Zealand, where casual sex ranged "from one-time sexual experiences with someone a woman had met that day, to a long-term sexual arrangement outside of a 'committed' relationship, to sex with an ex-boyfriend... the main element of casual sex was depicted as its 'uncommitted' nature".

We go into town to score someone. I guess it's that simple. You see someone that looks good and send out signals. If you're lucky you'll end up scoring [sexual activity] each other. You might swap details or find them on Facebook or something. And maybe get in touch with each other. Otherwise you just go back out the next week and find someone else. (Grant, SCV)

Within the narratives of casual sex is a strong undercurrent of a sexual double standard and dominant sexual scripts that are discussed later in the chapter. Here I consider it important to mention the "the complex and often confusing quality of consent" as the section presents young adults narratives of hooking-up, casual sex and alcohol use (Beres, 2007, p. 93). I am not going to go into detail on the complexities of consent - the definitional, theoretical and practical issues involved are discussed well elsewhere by writers such as New Zealand sociologist Melanie Beres (for example Beres, 2007 and Beres, 2014b). However, I do touch briefly on the complexities of communicating consent as young adults navigate in a more relaxed social context and how consent is understood and negotiated. 
In general, definitions of sexual consent are not consistent but the notion that young women, in particular, are free to consent or not to sexual activity simplifies the complex social context that young adults are engaging in. Strong social pressures were identified by the participants in this study where some unwanted sexual interactions were condoned or normalised and in some instances sex was consented to as a result of social pressure, either indirectly from dominant sexual scripts or from more direct pressure from a partner (Gavey, 2019; Beres \& MacDonald, 2015).

The concept of consent and unwanted sexual behaviour was captured in narratives on the normalised acceptability of unwanted touching, groping or kissing. Female participants described a level of acceptance within the hook-up culture of these types of sexualised behaviours as acceptable and in some instances this behaviour was a signal to young women that they were both desirable and attractive to men. While Emma described how "dirty" she felt by guys "constantly feeling her up", she also talked proudly about the frequency with which this happened signalling that she was "hot" and "attractive". Fileborn (2017) observed a similar occurrence and states that "unwanted sexual attention is also occurring within a cultural context where wanted sexual attention can be a welcomed if not desired feature of a night out” (p. 215). Likewise, Flood (2019) points to the pervasiveness of sexual harassment and states that within heterosexual norms the routine objectification of young women and the pressure young women feel to accommodate the desires of young men lead to beliefs that such sexual harassment is expected and normalised.

Young male participants also described how they routinely enacted such sexually invasive behaviours to get the attention of women and to indicate to those they found attractive that they were interested in them. Importantly, participants did not perceive these behaviours as unacceptable. Although in the violence literature these types of behaviours are labelled as "sexually aggressive", according to Becker and Tinkler's (2015) participants they represented a common and normalised range of behaviours witnessed and experienced in the bar and clubbing scene, and were not necessarily labelled as unacceptable. 


\section{WHERE RELATIONSHIPS BEGAN}

As a result of the drinking culture and engagement in the night-time economy most participants had met their current/ex-partner while socialising with friends in bars and getting drunk. Two participants described how they went home with a person they had hooked up with and then they "just stayed around" which eventually developed into a 'steady' dating relationship.

I guess hooking-up is common. You find someone, go home with them and then the relationship just goes from there. I mean, often the person is a friend of a friend so it's not completely random. (Sarah, SCV)

We met at a friend's drinks, a group of us went into town together and then we fell into bed together. There was lots of attraction. I guess after that it was easier for her to just stay at my place. It was convenient. (Jack, SCV)

While the majority of participants reflected the hooking-up culture, two participants described more traditional courting behaviours where they met their boyfriend as part of a group and got to know each other, eventually forming a romantic relationship once they decided that they had enough in common and there was sufficient attraction. Both participants discussed the need to get married before they would live with an intimate partner, complementing their strong religious and cultural beliefs.

We had met each other through friends, so we spent a lot of time together as a group hanging out. I got to know him quite well. We started hanging a little bit more on our own and eventually we started dating. It's not like some of my New Zealand friends though, we don't spend much time together alone, like we're in public when we're together, and we don't get too affectionate with each other. For us it's not acceptable, we will get married before we sleep together. (Sue, SCV)

This was consistent with comments made by New Zealand researchers Pryor and Josie (2005, p. 24) where they observed that only "a minority of couples get married before living together, and they usually bring with them the external structures of religious beliefs or family and social expectations". 


\section{ROLES IN ESTABLISHING RELATIONSHIPS/HOOKING-UP - ROMANTIC}

\section{ENCOUNTERS}

Within the narrative on the culture of hooking-up and the establishment of dating relationships there was also a strong narrative on the roles that males and females needed to enact in the dating process. In particular, the acceptable scripts and roles they must uphold and the social norms they felt they needed to adhere to, where men were expected to hold more "masculine agency" and women take on a "traditional feminine passivity” (Eaton \& Rose, 2011, p. 845). Sexual scripts are described as providing cues to how individuals or "actors" navigate through sexual situations and the appropriate behaviours to engage in, including the roles in initiating sexual interactions and the order that behaviours occur. While there are dominant sexual scripts, these vary across individuals and cultures, and these scripts are not necessarily "mutually exclusive or exhaustive” (Beres, 2014a, p. 83).

Within these varied sexual scripts, some researchers, such as Beres et al. (2019), describe shifts beginning to take place around the traditional roles of initiating sexual relationships, moving towards more egalitarian sexual scripts. However, they question how far these less traditional initiation patterns hold alongside strong underlying concepts of masculinity. Baker and Elizabeth (2013) state that it is still widely held that men are expected to initiate relationships and control their progression through to marriage. They labelled the pressure that young females felt to adhere to acceptable and more traditional dating scripts as a 'double standard of sexuality', arguing that women who initiate romantic encounters are viewed negatively as sexually aggressive. As one of my participants said:

If you like a guy you can't just go up and come on to him. You have to do things to get his attention and get him to initiate it, otherwise you just look real desperate, and then if something does happen you don't know if he really likes you or just thinks you're easy. (Kathryn, SCV)

The distinct gendered behaviours surrounding dating positions men as initiating relationships while women are limited to responding to the men's advances (Lamont, 2014). As Beres (2014, p. 77) states, while there may be evidence of shifts occurring in the gendered nature of sexual scripts: 
...many researchers argue that normative scripts place men in the position of initiator for dates and sex and women in the role of gatekeeper, who ultimately decide whether or not and when a couple engage in sex (Byers and Heinlein 1989; Dworkin and O'Sullivan 2005; Laner and Ventrone 1998; O'Sullivan and Byers 1992; Weaver and Herold 2000). These roles place men in positions of power during the early stages of "courtship". Men are reported to ask for dates and initiate kissing or invitations for a second date.

Lamont (2014) discussed how normative sexual standards are more strongly applied when there is uncertainty or where reassurance is needed, making the dating period, when the relationship is in the early stages of establishment, a time where young adults may be more likely to follow traditional gendered scripts. Likewise, Eaton and Rose (2011) discussed the need to transform gendered power patterns to allow more equality in relationships, but also noted that this is likely to be more difficult when young adults are trying to conform to acceptable dating scripts to ensure they make a good impression.

Even for participants who viewed themselves as egalitarian and who sought this in their relationships, there was a strong narrative around dating scenarios where the male was expected to be assertive and initiate casual sex and other dating interactions while females were described, by themselves and male participants, as passive players (Lamont, 2014).

I really liked him but it's just not cool to be all over him and ask him to dance. So I just played it cool, flirted heaps and made sure I looked hot. Eventually he got the hint that I was interested in him and wouldn't turn him down. He asked me if I wanted a drink and it went from there. That's a better story to tell than one where I sounded desperate, I mean, you just don't know if they really like you then. They need to make all the moves. (Rebecca, SCV)

In summary, participants described the almost universal culture of engaging in 'hookups' with relaxed attitudes to casual sex. However, within this culture there was a strongly perceived requirement to maintain gendered scripts when initiating and progressing through a relationship. As such, there was a need for participants to manage expectations of engaging in romantic encounters and forming relationships 
within cultural norms of what is appropriate, which appeared to be grounded in traditional gender roles and double standards.

\section{REASONS AND MOTIVATIONS FOR BEING IN A RELATIONSHIP}

Throughout the interviews, participants described different motivations for wanting to be in a romantic relationship. For most participants, as in the dating literature, participants described positive themes including being able to do things as a couple, the security of having someone around, and feelings of companionship, closeness and intimacy (Allen, 2004).

However, underlying some of the more positive motives for establishing a romantic relationship were more negative and harmful beliefs on the need to be in a dating relationship. Female participants described the cultural pressure to be in a relationship even if it was a negative relationship, as well as the need to be in a relationship to gain self-worth and to legitimise sexual relations. This was a gendered expectation. In contrast, male participants held dominant narratives around being single and out for a good time, especially as there was strong agreement from participants that it was far more acceptable for males to be single and have multiple sexual partners. However, some young men in the study did describe their desire to find someone special and to eventually be in a long-term relationship.

\section{Pressure to Be IN A RELATIONSHIP OR ACTIVELY LOOKING FOR A PARTNER}

Participants also described pressure to be on 'the lookout' for a partner even if meeting someone was not a personal priority. The main narrative for female participants around feeling the need to be in a relationship was based on a dominant romantic narrative that defined successful adulthood by being in a romantic relationship (Bay-Cheng \& Goodkind, 2016). Young women who were not in a relationship stated this reflected on their ability to fulfil and conform to traditional gender roles (Power, Koch, \& Jackson, 2006). Towns and Scott (2008) extend this narrative from their work on the 'culture of cool' and found that:

For some young women within this culture it is cool to be a girlfriend, being one provides a girl with social standing and allows her to be popular. (p. 75) 
For female participants this societal pressure was most strongly supported by their peers who they described as wanting them to be out and actively looking for a partner so that they would be in a relationship and not be alone. Such pressure was founded in a belief that being in a relationship would "make them happy".

We would go out and see people pashing [kissing], there is such a load of pressure to hook-up. I guess I felt cynical to others hooking-up, because there is this expectation that you need to find some guy and hook-up, everybody is on the prowl. (Rebecca, SCV)

My friends wanted me to go out with them and to try and hook-up with a guy. Like somehow they think a relationship will make me happy. That I need a man to make me complete. You know, like I can't be single and alone and happy. Even though I was happy being single my friends made me feel like a loser that I couldn't find a guy. (Emma, SCV)

This narrative is consistent with Allen's (2004) observations that young people's behaviour can often be explained through the influencing factor of peer pressure. Therefore it is reasonable to assume that peer pressure is a strong motivator for some young people to feel they need to engage in dating relationships to assimilate with group norms and to fit in with peer expectations.

\section{GAINING SELF-WORTH AND VALUE FROM BEING IN A RELATIONSHIP}

While there was strong peer pressure to socialise publicly, and to be seen to be looking to engage in romantic encounters, another driver for female participants was the desire to have a boyfriend. In this context, having a boyfriend provided a sense of self-worth and self-esteem, as a romantic relationship represented desirability and successfully conforming to cultural norms.

I've always wanted to have a boyfriend, to feel wanted. You just know it's part of being a grown up, and it's cool. And yeah to feel wanted. (Beth, SCV)

For two female participants, the perceived inability to find a romantic partner led to reports of feeling depressed as they associated being in a relationship with their own self-worth and self-esteem. In this sense the dual attainment of self-worth and selfesteem were reported as a strong driver for these young women to be in a relationship. 
Jackson (2001) discussed the inextricable link women describe between love and sex, which was consistent with some female participants in the current study who talked about how sex represented the male wanting them and a tool for their self-validation. Having sex was described as an element that defined one's worth and desirability, as illustrated by Sarah.

Interviewer: So sex is an important aspect of the relationship for you?

Sarah: Yeah, I feel good and, like, get self-esteem from having sex because it makes me feel wanted and all that stuff so if I don't get much sex then I don't feel that good about myself.

Interviewer: ... don't feel good about yourself, can you explain that a bit more?

Sarah: I just want the attention and to feel wanted. But my boyfriend is a big boy and so he doesn't always feel that good about himself and so he doesn't want sex but then that makes me feel crap too, that he doesn't want me. So if we're not having sex then we cuddle but it's not enough. I didn't know if it's me he isn't interested in or just because he had his own issues.

Interviewer: Do you talk about it with him?

Sarah: Nah, it's too awkward and he might tell me I'm useless in bed and that would destroy me. So I just hang out and wait for him to initiate it [sex].

(Sarah, SCV)

Another participant described how she and her partner were not having much sex and that if she initiated sex he would not respond. This led her to feel worthless and she talked about how her partner's rejection lowered her self-esteem and became a driver for her depression and alcohol abuse.

I've pressured him for sex, not in a bad way. But we have a weird sex life, like we only do it once every two weeks because he's so busy. Which isn't very much. We had code signals to each other like "how you doing" and he often says "I'm tired", so that means no sex. I guess the code is there so I don't get disappointed if I make actual advances. Even still, when he says no I get 
disappointed, I feel like I'm not good enough, not pretty enough. One time I broke down crying when he turned me down, he just, like, didn't even notice. I guess that shows that he does think I'm ugly and not worth making the effort. The constant rejection made me just feel shit, I started drinking all the time and hit rock bottom, like totally depressed and wanting to make the feelings just go away. (Mary, SCV)

This was a similar theme reported by Beres et al's (2019) study on the changing context of heterosexual sex. They found that within the belief that it is natural for men to want sex, some young women described men refusing their sexual advances as self-deprecating, creating a personal judgement that they, as young women, were not good enough.

\section{RELATIONSHIP REQUIRED TO LEGITIMISE YOUNG WOMEN HAVING SEX}

Another narrative from female participants was the importance a romantic relationship played in their need to legitimately engage in physical elements of a relationship (sexual relations). Jackson (2001) discussed how, for females, relationships can legitimise sex and serve as a metaphor to justify a women's own sexual desire. Jackson's analysis was consistent with some female participants in the current study who held a strong narrative around needing to be in a relationship to legitimise sex. For some females they stayed in a relationship simply to have access to sexual intimacy without being viewed negatively.

It's true for some girls, that they do want it [sex] but girls you have to catch. Society makes girls feel like gatekeepers and not to be easy, but they do want it. Females want it but have to hold out otherwise they're seen as sluts. (Mary, $S C V)$

Girls give a lot, guys just take. As a girl you can't go out and sleep around because you look like a slut so this feeds the need to be in a relationship rather than look like a slut. It provides a cover story "he's my boyfriend so it's okay for me to be having sex". For guys it's easier to sleep around, but it's good if you have one permanent [girlfriend] on tap because she's going to give it without the guy trying too hard. (Rebecca, SCV) 
Participants described how females who had multiple sexual partners without being in a steady relationship were perceived negatively, commonly attributed with labels of "sluts" or "easy", or "not a good girlfriend prospect". Both male and female participants described how it was far more acceptable for men to have multiple sexual encounters with different women outside of a relationship. Effectively, a societal discourse was reported as permissively treating males having multiple sexual encounters as acceptable while for females this was not the case.

This is what Farvid and Braun (2014) term as an enduring double standard towards women's casual sex where social norms make it acceptable for men to have casual sex, while for women casual sex is positioned as problematic and even dangerous. Beres and Farvid (2010) describe how this sexual double standard can result in women being labelled as promiscuous when engaging in casual sex and if they experience negative consequences in the context of casual sex, they are blamed for this. Baker and Elizabeth (2013) also discuss the double standard of sexuality and although they argue it has eroded over the past few decades, the ability for males to more legitimately participate in non-monogamous sexual relationships still appears to be far more acceptable.

There's a coolness factor for guys to say they've been with a lot of girls. Girls not so much, if they hook-up with loads of different guys they look like sluts. (Kathryn, SCV)

Participants described the acceptability of males having multiple sexual partners as cached under the banner of 'being a bloke' and 'getting the bachelor out'. Farvid and Braun (2014) described this social norm in their analysis of men's magazines that support this cultural script:

The modern man should be able to act in any way he pleases, free of feelings of guilt, depression, anxiety, doubt or ethical complexity. Masculine subjectivity was thus constituted as carefree, careless, and uncomplicated. (p. 120)

In fact, male participants described how it was often expected that men should 'brag' with their mates about their romantic encounters, where "sex has become a set of 
achievements for men, a means by which men perform masculinity and heterosexuality” (Farvid \& Braun, 2014, p. 124).

While it appeared, from participants narratives, that it was far more acceptable for young men to engage in casual sex and sex outside of a formal relationship, one male participant described how he would treat a young woman differently if he was "really serious about her". Jack described how he would engage in more casual romantic encounters with girls but when he met a girl he was serious about, he took a more traditional approach in how he established the relationship with her. This included taking things slow, getting to know her and showing a greater level of respect.

I took a more traditional approach when I met a girl that I was really infatuated with. I wanted to do everything right. I wanted to get to know her, not just fall into bed. I was so intrigued with her. I wanted the romance to be memorable, I cared about it more. (Jack, SCV)

While Jack talked about how his behaviour changed in the context of establishing a relationship, two male participants also described pressure to get male peer approval by overtly displaying a need for sexual activity, "lots of it, all the time".

Demonstrating a constant appetite for sex did not reflect their actual desire for and beliefs about sex. This is highlighted in the current study by Drake who described putting on a front, using sexualised language and machismo-derived behaviour to appear "manly". Of interest, neither male participant would follow through with the behaviours 'expected' of them. These contrasting narratives highlight the notion that there is not one standard set of ideals; in fact, there are multiple masculinities operating with some sexual scripts deviating from traditional attitudes and behaviours towards how men initiate and engage in experiences of sex (Beres et al., 2019).

There's this view that guys are always wanting it but they don't, so they cover it up, like saying "she's ugly..." so you make it about the girl to hide that you don't want sex. There's just pressure to always want sex. Guys are pressured to be macho and not doing gay things. Macho guys overdo it, they say what they think they need to say but it's over the top. Lots of guys value family and respect girls but have this front of being macho. (Drake, SCV) 
Interestingly, while male participants talked about the acceptability from peers to have sexual partners outside of a relationship, they also discussed how they had to carefully balance an image of being a bloke and wanting sex with not being seen by females as a player, otherwise they would lose their status as being a 'viable' dating option. As Chung (2005) states, conforming to gendered expectations inside of heterosexual relationships for men can mean that potential girlfriends can be turned off by a male if he is deemed too promiscuous, meaning that they do not trust them. In the long-term this can interfere with a male's ability to conform to the need to eventually engage in some form of more enduring long-term relationship, which is also a function of performing successful masculinities in later life. As Beres et al. (2019, p. 128) state, "this will mean that they will take up various masculine positions (and even, at times, "feminine" positions) according to the needs of a given interaction, most often with reference to other men".

I've had a lot of girls, you know what I mean? And that's cool to brag about with my male buddies but then you have to make sure you play it down around chicks. You don't want to look like a total dog, then no one will get with you [engage in a sexual activity]. It doesn't make you good boyfriend material. (Regan, IT)

This section described participants' motivations to be in a romantic relationship. For most of the participants, the motives for being in a relationship were positive, including having companionship and intimacy. However, there was a strong narrative from female participants about the pressure from friends to be in a relationship, even if it was a negative relationship, and the need to be in a relationship to gain selfworth, and to legitimise sexual activity. As Jackson and Vares (2015b) write:

... young women are subjected to constant surveillance and regulation, granted sexual freedoms on the one hand but attached to regulatory moral conditions of white middle-class respectable femininity on the other. (p. 482)

A double standard also existed, where female participants' described the need to be in a relationship to legitimise casual sexual activity, while this was not viewed as required for male participants. 


\section{LIVING TOGETHER AND THE ROLES IN THE HOUSEHOLD}

Western society has seen a dramatic increase in the rate of cohabitation with the Organisation for Economic Co-operation and Development (OECD) reporting that over three-quarters of couples now cohabit before marriage (OECD, 2011 in Baker \& Elizabeth, 2013). Throughout the interviews, most participants reported that living together was not seen as a reflection of the seriousness of their relationship but more often a result of circumstance or convenience. Consistent with previous research (see Baker \& Elizabeth, 2013), the current study found that moving in together was not necessarily a proxy for seriousness but was often viewed as being "convenient" or "making common sense", such as one partner not having a place to live, to share costs, or to be closer to each other.

We just ended up living together. He was flatting and there was a spare room so I moved in. I needed somewhere to live so it worked out great. It was just convenient, good timing really. (Beth, SCV)

It kinda just made sense, we were hanging out heaps at my place and there wasn't much point in us paying rent at different flats, especially since he was never at his place. So he just moved in. (Emma, SCV)

Participants commonly described a less traditional experience where they ended up living together while dating, and often from quite early on in the relationship, or as Baker and Elizabeth (2012, p. 12) explain, young adults "incrementally slide into cohabitation with little actual decision making or enhanced commitment". Very few participants talked about cohabitation being a conscious decision made together based on relationship seriousness.

He just came home with me one night and didn't leave. We were together all the time, it just happened naturally. I don't think we really thought about it being a serious thing, it just meant we could hang together. (Grant, SCV) One night while I was really drunk I said to him "when will you ask me out". So he did, and I said yes. A few nights later I fell asleep at his place so I stayed the night, he didn't know what to do. Then essentially we lived together from then. (Mary, SCV) 


\section{TRADITIONAL GENDER ROLES}

Participants' narratives highlighted the shift in the acceptability and normality of living with a romantic partner outside of relationship commitment, seriousness and marriage. Although participants described a less traditional establishment of dating relationships and a high level of cohabitation, the roles within dating relationships quickly established a more traditional gender-based ideology where gender roles were adhered to, contrary to conversations about equality. Lamont (2014) discussed how women primarily seek egalitarian relationships where work and home responsibilities are shared, although Lamont (2014) noted that women were still often carrying out the majority of the household chores.

Participants described the desire for an egalitarian relationship in and out of the household, but female participants' narratives quickly shifted to describing situations where they adopted roles "like their boyfriend's mothers", indicating that they often took on the more traditional gender-based roles in the performance of gender.

Sometimes I felt like his mother - I cooked, cleaned, did all those jobs. Ifelt like his mum not his girlfriend. It just developed into those roles, I tried to change this but it just led to arguments. (Rebecca, SCV)

Ifeel a bit like his mum - his mum cooks and cleans all the time so he has expectations that I'll do this. And so I do a lot of cooking and cleaning now. I feel domesticated but a bit too much. (Beth, SCV)

He was so nice and so amazing but we were living together and I was like his mother doing all the cooking and cleaning, washing. He never took me out we just stayed home and I did all the chores while he sat and watched TV. Eventually it became set in stone, it developed into those roles. And I couldn't get out of that routine. (Sarah, SCV)

Lamont (2014) discussed the "moral dilemma" that results from seeking equality in dating relationships that competes with the traditional gender-based ideologies that strongly persist for young adults. Such positioning presented female participants with a moral dilemma. Counter to narratives of wanting egalitarian relationships, most participants described their desire for more conventional courtship behaviours when 
the male would pay for the first date, would "ask them out" (make the relationship exclusive) and would enact 'chivalrous' gestures such as buying them flowers. These acts of chivalry often represented to a woman that the male is respectful, caring and not just wanting a sexual encounter (Lamont, 2014).

It's still right for the guy to buy you drinks, pay for dinner and that sort of thing. It's sort of up to them to ask a girl out and then do the right thing of looking after her. I would always offer to pay but would expect that the guy would insist on paying. It's just how it should be. (Sue, SCV)

This section has presented a narrative of participants' around the attitudes, values and beliefs that they navigate within the modern New Zealand cultural context and the way young people socialise and form romantic relationships within it. The contemporary social context that was described by participants was embedded in a dominant drinking and hooking-up culture where it was largely accepted for young adults to go out partying and get drunk.

Participants described that they felt there was also a strong expectation that young adults, particularly young women, engage in romantic encounters and form romantic relationships within the cultural norms of what is appropriate, which appeared to be grounded in traditional gender roles and double standards. The motivation to be in relationships was driven by perceived expectations that relationships were part of achieving adult status. For female participants, a romantic relationship had a stronger incentive as it was viewed as a way to gain self-worth and to legitimise sexual activity.

However, there was also an additional influence that was strongly associated and interacted intimately with by participants, and that was through the growth in access and the use of technology, social media and popular culture. The extent of this influence warrants its own section and is described next.

\section{SECTION TWO: IMPACT OF TECHNOLOGY, SOCIAL MEDIA AND POPULAR CULTURE}

This section focuses on the role of technology, social media, the internet and wider media in influencing the social norms and expectations as described by the young 
adults in this study. In particular, this section will focus on how technology and wider media influence what is acceptable to wear, how to act, and how to be in a relationship and how it drives the constant scrutiny by others regarding how young adults measure up to these set norms and expectations.

\section{THE ROLE OF TECHNOLOGY, THE INTERNET AND THE MEDIA}

All participants described the proliferation of modern technology, the internet and social media, in both positive and negative ways. Mobile phones - in widespread ownership - along with sites such as Facebook, were described as standard tools used in social and dating interactions. All participants referred to texting mates, following people on social media, using social media to meet people, to stay in touch and to know what social events were coming up. It was an important tool in how these young people interacted and socialised. Technology was also widely used by participants in the establishment of a relationship, as ongoing communication during relationships, and even in ending a relationship (Baker \& Carreno, 2016). Technology was not used by participants just to stay in touch and keep connected but also as a mechanism to access information and education in all aspects of their lives, including general day-to-day information, and to support work and study. It was also hugely influential in setting group norms and what was "cool" and "in" (Konstam, 2019).

Social media was discussed by participants as setting norms by influencing how they thought they should look when they were out socialising, to fit in and maintain group norms, how they should act when out with friends, to inform them about what a relationship should look like and how they should behave with their romantic partner (Konstam, 2019). Participants commonly described how the wider media itself was a constant source of entertainment and education with advertising and media in New Zealand promoting an "unquestioned and accepted" ideal for young people (Towns \& Scott, 2008, p. 75). The proliferation of media not only created unrealistic ideals on how they should look and behave, it was also a mechanism by which harmful attitudes and beliefs were established and reinforced how young men should treat women in relationships and the acceptance of violence in dating relationships. As Hutton et al. (2016, p. 76) described, social media sites have become a key way of communicating for young adults, but they are also places where "social relationships around gender, ethnicity and class are performed and negotiated". 
Participants described how group norms were strongly influenced by social media and wider media including television, the internet, magazines, reality TV and reality stars. Emma summarised the influence of technology and media in her comments on how she "knew" what to wear to maintain cultural and peer norms.

Shit, I am always on social media to see what people are wearing, what celebrities are doing. That's where you can see what's cool to wear, what's cool to talk about. My group of girlfriends spend a huge amount of time making sure we're dressing right and looking amazing. You have to keep up to be part of the group. (Emma, SCV)

Conversely, modern technology, the internet and social media were mechanisms that caused participants to set unrealistic expectations of self-image and relationship experience. It influenced behaviours that encouraged participants to develop a false image and also allowed greater monitoring and surveillance of each other. This is described by Manago et al. (2015) where young adults curate flattering images of themselves and post images that are sexually provocative to gain attention of both the same and opposite sex. They state that young adults are constantly assessing what their peers are engaging in, gauging social norms and assessing their standings in relation to peers through their engagement and use of social media.

Participants felt that through social media, their peers set expectations on how they should look and behave, therefore a very common narrative from participants was how important it was for them to present an image of "perfection" so that friends and unknown audiences would see them in a socially acceptable way. The prolific use of the word "perfection" was seen in Jackson and Vares' (2015a, p. 352) research where the word 'perfect' was used by young women who implicitly "recognised perfection as a normative requirement of femininity".

Both male and female participants talked to varying degrees about the pressure they felt to create a false image through social media where they appeared happy, looked good, were out socialising and, when they were in a relationship, the importance of using social media to create the appearance that they were in a perfect relationship. Such talk demonstrates the way social media usage has to be carefully managed to ensure the most ideal visual representation (Hutton et al., 2016). 
It's the angle you take a photo from, what drink you have in your hand, where you're hanging out. You have to make sure you get the right look so people see you as being cool and on trend. (Beth, SCV)

There's always someone with a camera or posting something so you always have to make sure you're looking good. And I always look at the pics that people tag me in, if I look like crap then I will un-tag myself or delete the pic from my feed. A few times I've made chicks take down photos, they just aren't a good look for me. You have to maintain an image. (Jack, SCV)

The most common narrative was within the context of going out socialising and engaging in the night-time economy. Participants described using social media to create a perception of having a good time but at the same time not being "out of control". In other words, participants needed to negotiate the right kind of online image while at the same time be seen out engaging in 'appropriate' drinking to gain social capital and belonging to a peer group but not broadcasting any behaviours that could be "labelled as promiscuous or disreputable” (Hutton et al., 2016, p. 77).

\section{SELF-MONITORING AND SURVEILLANCE TO MAINTAIN AN ACCEPTABLE IMAGE}

Essentially the narrative of broadcasting appropriate images via social media led to participants modifying and sanitising their behaviours and appearance and placed pressure on them to self-monitor. The impact of this was that participants critically assessed what they posted to social media to maintain an appropriate appearance. Jackson and Vares (2015a) discuss this need for young women, in particular, to participate in their own self-surveillance to achieve an aesthetic that fits with wider societal expectations of appropriate performances of femininity.

Similarly, male participants also described a need for self-surveillance although this was motivated by the need to conform to strong notions of masculinity, to be "one of the boys", balancing that with the need to maintain a certain level of behaviours that would still make them desirable to females, and maintaining a traditional image of being a suitable partner.

You have to show that you're out partying and being one of the boys, ya know, having a good time, drinking, scoping out chicks. But you can't look like too 
much of a dog, you don't want chicks to think you're such a male slut that they don't want to hook-up with you. (Ricky, IT)

The pressure to portray the right image was described by participants as part of being involved in the social environment and although it did cause stress for some participants, modifying and sanitising their behaviours was not particularly harmful. However, some participants described more serious impacts from viewing the sanitised versions of their friends' experiences of the night-time economy.

Hutton et al. (2016) described how the false image that young adults portray through social media, by not showing less acceptable behaviours such as vomiting or becoming aggressive, can impact on the behaviours of other young adults as they perceive that there are no negative consequences to the excessive 'fun' drinking experience. This unrealistic perception can lead to a dangerous situation where young people can drink to excess, black out, and become increasingly vulnerable.

One participant described how they viewed a peer's behaviour online and emulated this behaviour. This led to a harmful situation of excess drinking resulting in his admission to hospital.

My mate always posts pics of his big nights, a line-up of empty bourbon bottles and beer, and he always looks like he is having a blast. I figured I could handle that much alcohol, so I just skulled drink after drink. I don't really remember what happened but apparently I was pretty aggressive, got kicked out of the pub and passed out on the street. I smashed my head open. It was pretty messy. I got taken to A\&E. It was pretty shameful, like I couldn't handle it at all like him. (Regan, IT)

\section{EXPECTATIONS OF WHAT RELATIONSHIPS SHOULD LOOK LIKE BASED ON PEERS'} SOCIAL MEDIA PRESENCE

While participants talked more generally about the need to create an acceptable selfimage on social media across all aspects of their lives, including appearance, drinking activity, and socialising, a particular focus for female participants was the use of social media to see what other romantic relationships looked like and to compare what they were experiencing in their own romantic relationship. Although participants 
knew it was likely that what they were seeing was a sanitised version for 'show', they still felt the need to match up to these unrealistic expectations.

You live relationships quite differently with social media. You use Facebook to make relationships look perfect but actually it's not reality. But you gotta save face 'cause you have 1,000 Facebook friends. (Jessica, IT)

Knowing that peers would be viewing their online presence to assess and judge each other's lives also created a culture where participants felt like they could not be honest about the realities of daily life and relationships. This made it hard to create dialogue with friends around their negative experiences, leading to young adults staying in bad relationships longer to avoid admitting they were not in a perfect relationship.

They've got such a perfect relationship, they're such a cute couple, they've been together forever. You kinda want people to say that and not that they are such a disaster and should break up. It's all about putting that front on. I just wish I'd been more confident to end things sooner and not to be such a coward. I was just ashamed that I'd made it look so happy and great but really I was miserable but couldn't be honest about it. It was a real trap. (Rebecca, SCV)

On the whole participants appeared to spend a significant amount of time and energy establishing the perceived expected way to act and negotiating group norms in all elements of their daily life. Their juggling of appearances and expectations was a constant pressure.

MEDIA'S ROLE IN HOW TO ACT, DRESS AND WHAT RELATIONSHIPS SHOULD LOOK LIKE

Aside from peer influences, participants were also strongly influenced by popular culture and wider media which appeared to more explicitly and purposefully set up unrealistic expectations of what to look like, what to buy, what a relationship should be like, and the roles young adult men and women should play in romantic encounters and dating relationships. 
As Jackson (2001) described, from a young age women are constantly confronted through music videos, television shows, reality television, teen fiction, magazines and advertising with "romantic narratives" that create unrealistic ideas of what relationships should look like and how women and men should behave in a relationship and the roles they must play. The issue is that what is being portrayed and the stories told through media and pop culture are exaggerated and hyped, thus creating unrealistic expectations for young adults.

A number of participants described how the wider media portrayed the ideal relationship as hot and glamorous, placing pressure, on women in particular, to create an outward appearance of this in their own lives, and driving a second image that was not how they actually felt or how they wanted to behave. Beth described how most of what young people are exposed to daily creates unrealistic expectations and adds to the pressure to look perfect, act in a certain way and to be in a relationship.

Constantly you are being sold this story of what relationships are like. If you don't stop and pay attention you start to believe that it's reality or something. You can't look like a model, have a boyfriend that is some kind of perfect, always be smiling in the latest styles, on some beach. As if that's possible, and so you are made to think that your relationship sucks 'cause it's not like that. That you're not enough. I'm not saying my relationship with Ethan was great but it was never going to be all sunshine, sex and six-packs. (Beth, SCV)

The unachievable ideal created by media set some participants on a path to acquiring a certain look, to act in a certain way and educates them on how they needed to behave to secure a romantic partner, and how to behave in a romantic relationship. This placed pressure on participants to continually seek to reach this standard set by the media. However, these unrealistic expectations and unobtainable standards lead to continual disappointment as these participants found themselves in relationships that seemed less than satisfactory and where they never found their Prince Charming.

Most of the female participants described continued disappointment from being in relationships that did not measure up to expectations driven by social media and the wider media. Female participants discussed that although they felt that the 
relationships on reality television and portrayed in the media were glamorised, it set up a desire for something that was likely unrealistic and unattainable.

I often think maybe our relationship is ok, maybe this is good and I want something that isn't real. But how do you know that, how do I know if I should expect more or if this is enough? I'm supposed to be swept off my feet and infatuated but I'm not, but maybe that's ok. It's really hard to know. (Sarah, $S C V)$

I still have hopes that a movie ending will play out. I guess I'm just waiting for my Prince Charming, but at the moment I'm just aiming for good. (Rebecca, $S C V)$

\section{MEDIA SUPPORTING AND GLAMORISING CONTROL AND VIOLENCE}

In addition to the influence and impact of different forms of media and technology on setting expectations, a number of participants talked in detail about the negative impacts that media had on supporting violent behaviours by normalising control and glamorising violence.

Participants reported the common occurrence of physical aggression and violence being depicted across all forms of media, whether it be in movies, television shows, music videos or YouTube clips. Within this media-context is the representation of love and romantic relationships that occurs in the context of jealousy, control and violence (Breger, 2017).

The representation of romantic relationships in the media depicting harmful behaviours as normal in the context of love can cause young adults to conflate this behaviour with intimacy and love (Papp, Liss, Erchull, Godfrey, \& WaalandKreutzer, 2017). This narrative of seemingly loving and chivalrous behaviours being reinterpretations of control and ownership were also picked up in participants' descriptions of celebrity relationships they saw reported in the media.

At the time of the interviews there was a significant focus in the media and gossip magazines on the new relationship between celebrity Kim Kardashian and rapper Kanye West. Without prompting, four female participants discussed this celebrity relationship. The latest story had been about Kanye throwing out all of Kim's clothes 
and buying her new clothes that he wanted her to wear. The participants were conflicted between seeing this behaviour as chivalrous and romantic or as what can be described as, the performance of toxic masculinity as Kanye was telling Kim what to do and controlling her appearance. As Kathryn processed her thoughts she began to see how his seemingly generous gesture could actually be viewed as controlling behaviour.

Kathryn - It's so glam, to have anything you want, look the way you want. Like for Kim he just bought her all this stuff. He didn't like what she wore so Kim talked about how he went through her wardrobe and chucked the stuff he didn't like and then went and spent all this coin on new stuff he liked. I mean, that's pretty sweet.

Interviewer - so he got rid of clothes he didn't like?

Kathryn - Yeah, it just sounds really cool, someone spends all that money on you to look good. I'm guessing she had some say in it. Otherwise it would kinda be a little crap. It wouldn't be cool if he made all the decisions on what she could do if she didn't want that. That's how the story sounded, that it was ok for him to do that but maybe it really isn't? If it was a way to control her or make her be someone she's not. Who really knows? (Kathryn, SCV)

Another significant story in the popular/celebrity media at the time of the interviews was Chris Brown's (singer) assault on his girlfriend Rihanna (singer), his subsequent arrest and conviction. At the time of the interviews they had re-established their relationship and were reported as dating. Several participants discussed this story when talking about what violence was in a relationship.

There's been lots about how Chris Brown beat Rihanna. There was a picture that was taken of her face. It looked horrific. The picture was on the front of all the magazines. It would've been a good opportunity to write some quality article about how this behaviour is not ok, but some of the stories, the comments on social media show that we're a long way off quality reporting. Implying that it was her fault for upsetting him, that he'd come from a bad background but was reformed and this was just a one off. There's no excuse and the way that headlines run and magazines get you to read them is by 
reporting stuff like this, hyping it up. It doesn't help in educating people about what's really ok and what is never acceptable. (Beth, SCV)

What was evident from discussions was how the media went from reporting their disgust at the violence that Chris Brown used, including publishing pictures of Rihanna's bruises on the front covers of their magazines, to then seemingly supporting them re-establishing their romantic relationship together and using language that minimised the previous violence. In fact, as participants described, the media's stories likely had little, if any, fact or truth behind them and in no way supported educating young people on what behaviours are not acceptable. Instead, quite the opposite was described, where the media became a catalyst of misinformation and helped to reinforce the myths and beliefs supportive of men's use of violence (Breger, 2017). Sarah described her reaction to the media stories:

The headlines, the stories they make out that a guy's violence is natural, that they can't control themselves, the media makes out like it's just men being men, it's appalling really, that were supposed to think that 'cause men can't control their emotions that it's ok for them to be violent and hurt someone they love, as if it is actually love. That's not love. (Sarah, SCV)

What this section presents is the proliferation and everyday interaction and influence of modern technology, the internet and social media, how it shapes attitudes and beliefs and drives behaviours based on false images to ensure young adults maintain image and status amongst their peers. Any programmes or interventions designed to promote healthy relationships and eliminate dating violence need to account for the influence and role that media and technology can play - both in a positive way (connection, forms of social movements and social good) or a negative one (misinformation, driving negative attitudes and beliefs).

\section{CHAPTER SUMMARY}

The main purpose of this chapter was to establish what 'dating' means for young New Zealanders and how this was constructed within the current New Zealand context and the broader global youth culture. In other words, to understand how the global culture that is evident in the New Zealand context plays a role in shaping the experiences of dating for our young adults as they move into more serious relationships and are at a 
greater risk of experiencing the negative impacts of violence in their dating relationships.

Overall this chapter presents participants views on a social context that has shifted dramatically from even just a couple of decades ago. What it has highlighted is that the contemporary social context that participants are navigating is complex as more modern expectations are magnified by technology and media, while competing alongside traditional expectations about the successful enactment of masculinity and femininity in the dating context.

This analysis is important to place the experience of violence encountered by these participants within their reality, which is embedded in the contemporary social context that was presented in this chapter. This helps to give context to the narrative on participants' experiences of dating violence that are described in the next chapter that will look to validate Johnson's (2008) statistically-derived categories through qualitative investigation. In doing this I will assess the validity and utility of his typology in understanding the complexity of dating violence experiences, assessing the extent to which this typology is a valid framework to understand the different experiences of violence in dating relationships. 


\title{
CHAPTER EIGHT - CONFLICT AND VIOLENCE IN DATING RELATIONSHIPS
}

\author{
You hit me once | I hit you back| You gave a kick, I gave a slap \\ You smashed a plate over my head | Then I set fire to our bed \\ My black eye casts no shadow | Your red eye sees nothing \\ Your slap don't stick $\mid$ Your kicks don't hit $\mid$ So we remain the same \\ Love sticks | Sweat drips | Break the lock if it don't fit \\ A kick to the teeth is good for some \\ A kiss with a fist is better than none \\ Lyrics from 'Kiss with a Fist' by Florence and the Machine
}

As established in Chapter Two, dating violence is a serious issue that appears to be commonly experienced by young adults as evidenced from both the international literature and through this study. The analysis in Chapter Five demonstrated this high rate of violence, with analysis in Chapter Six extending this to look at whether classifying such violence by levels of control provided a stronger distinction between experiences of violence. This chapter investigates whether there is a qualitative difference between Johnson's categories of violence. Participants were categorised as non-violent, intimate terrorism or situational couple violence using the analysis protocol described in Chapter Three.

This chapter focuses on the behaviours that were described by participants that were classified as dating violence. Dating violence can be enacted through a range of tactics including non-physical forms such as intimidation, emotional abuse, isolation, coercion and threats and physical forms like physical or sexual assault (Nybergh et al., 2016). Therefore this chapter is divided into two sections. The first section presents narratives on the experience of non-physical tactics and the second section presents narratives on the experiences of physical and sexual violence. 


\section{SECTION ONE: PARTICIPANTS' EXPERIENCE NON-PHYSICAL DATING VIOLENCE}

There are a range of non-physical tactics including coercion and threats, intimidation, and economic abuse. This section describes instances where participants talked about these elements and the context in which these occurred. I also discuss how participants experiences are consistent with Johnson's typology and the categories they were assigned to, as discussed in Chapter Six.

\section{MONITORING AND CONTROL THROUGH TECHNOLOGY AND SOCIAL MEDIA}

As described in Chapter Seven, participants were operating in a contemporary social context where there is a proliferation of technology and media, and within the context of violence these became tools that enable participants to monitor and control their dating partners (Baker \& Carreno, 2016). Participants described modern technology in both positive and negative ways. On one level technology was described as a mechanism to meet and stay in touch, for networking and support, but conversely it was a tool that allowed greater monitoring and surveillance of behaviours and even provoked additional violence (Henry \& Powell, 2016).

The proliferation of technology and social media, and the common experience of this by all participants as a tool for monitoring and surveillance of dating partners, was a central narrative in this study. The use of technology and social media outlets were a mechanism for such surveillance, a practice where people's actions and decisions are controlled "by making them accountable to another who will scrutinise and criticise their actions" (Towns, 2009, p. 31). Jessica illustrated this theme well as she described her experience of being monitored by her boyfriend during their relationship and how it led to violence.

He used to keep track of where I was at, who I was with, what I was doing. If I didn't respond then he would turn up to my house or the party I was at demanding to know what I was doing, he always assumed the worst. This one time I was tagged in a photo posted on Facebook with a guy and he flipped out because he'd come up with some made up story about what we were up to. Nothing happened but he flipped out. That was the first night that he choked 
me, it was petrifying. The sad part was that the guy in the picture was actually my cousin and it was totally innocent. (Jessica, IT)

Likewise, Laura described how her boyfriend checked up on her all the time and wanted to know where she was and insisted on picking her up from university, work and when she was out with friends. He would do this by contacting her on her cell phone and by monitoring her social media use and tags. At first Laura described how she interpreted this attention as a sign of his love and dedication to her, but when this behaviour became part of a range of other controlling behaviours she realised that his actions were a way to monitor and control her, and his acts were no longer interpreted as caring and chivalrous. The following quote shows how her boyfriend used social media and technology to monitor her. The quote also demonstrates how Laura viewed movies as a reference point that reinforced the representation of harmful behaviours as normal in the context of love, which can cause young adults to conflate this behaviour as representing intimacy and love (Papp et al., 2017).

He was always in touch, so much attention, like he cared, he was really sweet. It was like the movies, you know where the guy is there all the time, or like, knows what you've been up to, and you think that, like, that's cool that he cares. That kinda attention, that is what good boyfriends do. Then it switches into the stalker mode. He started doing other weird stuff like just turning up to work, or my uni classes, when that began the constant messaging and checking in became like as sort of, like chains, like I felt that I couldn't do anything without him knowing and there started to be bad things that happened when he didn't like what he saw, who I was with, or something. (Laura, IT)

These examples from Jessica and Laura represent monitoring and surveillance as part of a more systematic pattern of control, and in the context of an intimate terrorist boyfriend (this will be further described later in this chapter).

\section{Men MAINTAINing AN IMAGE OF BEING A BLOKE}

Another non-violent controlling tactic described by females classified as experiencing situational couple violence and intimate terrorism relationships was when they were forced to dress in certain ways to maintain a "picture perfect" image as the 
respectable girlfriend. As in Chapter Seven, participants described the pressure they felt to portray the 'right' image, particularly through their social media representation, but this also extended into a pattern of control when, in this case, some male dating partners required their girlfriends to look, behave, and represent themselves in a particular way to support their male status.

Rebecca explained how she recognised a gradual increase in control over what she could wear, to the point where she was not comfortable any more and felt like she was just there to be his "hot trophy girlfriend".

There's already mad pressure on girlfriends on how to look and act, that's what famous chicks and media do to us. Then on top of that Ben took a whole lot of my clothes off me because he didn't want me dressing like that, too dowdy. It did start with low key "change your way" statements; it wasn't real in your face. He just modified things a little bit at a time. It was easier to ignore at first. But then one day I didn't feel like me anymore, I wasn't wearing anything I felt comfortable in, it was stuff he made me buy so I would look hot. He just wanted me to be his hot trophy girlfriend, although there were some outfits I could only wear when I was out with him. He probably didn't trust me enough to be smokin' without him there to keep an eye on me. (Rebecca, SCV)

While Rebecca described her boyfriend dressing her to be a "show pony", Karen described how it made her feel when her partner forced her to dress how he wanted, to make her appear less desirable to other men.

Karen described how she put on an outfit and her boyfriend said she looked like a slut. She was ordered to remove it and wear what he picked out. Karen used the word 'ordered' to demonstrate her lack of ability to disagree and went on to describe how this behaviour was done to de-sexualise her and make her less desirable to other males. She still needed to look "perfect" so that his image was not tainted. Her image represented his status, that he had a desirable girlfriend but he did not want his mates to see too much of her body. She had to look "respectable". It was through his describing their "hot sex life" to his mates that he could demonstrate how desirable 
she was sexually, and how manly he was by being able to get her to "service him" and "pleasure him" when and how he wanted.

So yeah, he did want me to look like a respectable girlfriend, like still put together and not dowdy but not too hot. He got super jealous if other guys paid attention to me like that, I had to make sure I acted like that too, respectable, like not slutty. I stayed close by him and he would make comments and jokes to his friends, like through that he would imply that the sex was good, that I was good at pleasing him and all, you know what I mean? (Karen, IT)

Both these young women provided a strong narrative on their boyfriend's use of dominance to control their image and ensure, as good girlfriends, they supported his status of being "the man", and not doing anything to make him look bad. These young women described how they needed to behave and act like what their boyfriend defined as the perfect girlfriend to prove his ownership of them and to maintain his status amongst his male peers.

The practices evident in the young women's accounts indicate the development of power relations between the young woman and her partner that resulted in restrictions in her choices and her ability to act with autonomy. Similar to domestic violence, her usual practices were discursively reconstituted as gendered transgressions and requiring her boyfriend's control and authoritative intervention. (Towns \& Scott, 2013, p. 551)

\section{MEN CHANGE THEIR BEHAVIOUR IN PUBLIC VERSUS IN PRIVATE}

Tied with some female participants description of their boyfriends control over their image was the need for some male participants to ensure they conformed to the image they wanted to portray in order to maintain status amongst their male peers. Some young men were also described as modifying their own behaviour in certain situations in a way to control their image to others outside of the relationship, by creating an image of being a 'good guy'. This tactic, where they publicly modified their behaviour, served to discredit a partner's story of violence.

Two of the young women with male partners classified as intimate terrorists described how their boyfriends would make themselves look good in public to mask their 
violence and to discredit any attempt she might make to draw attention to his behaviour. Their boyfriends' ability to modify their behaviour in different contexts created a perception to friends and family that he was a 'nice guy'; this is what he was like in public so this is what he was like all the time. In this way friends and family became part of the system that supported his acts of control, further trapping the young woman in the relationship where she doubted herself and her reaction to his behaviour. For instance, Karen described how her boyfriend was 'lovely' around her friends but acted as a 'monster' after they were alone. Her friends felt she was exaggerating her complaints of his controlling behaviours since he was "such a nice guy".

Karen - He put on this show, like he could be real nice, almost a completely different person when we were out with mates or with my family. It was pretty amazing. My friends adored him; he was so charming and sweet, I mean that's why I liked him to start off with. But then it just got creepy and he was really controlling. He just did things that made my skin crawl, they were normal as, I guess, unless you knew what he could really be like.

Interviewer - Can you give me an example of this?

Karen - Yeah I guess, um I went out one night with my girlfriends for dinner and he was clear that I had to stay at the restaurant and go nowhere else, and that he would pick me up. My friends thought it was so sweet that he let me go out for the night and that he'd go out of his way to pick me up instead of catching a bus. I knew it wasn't cos he was being nice, he just wanted to make sure he knew where I was and who I was with. It was all about being the boss. But my girlfriends didn't see it. I mean, maybe I was just being a bitch, that's what I thought, maybe I was being a brat. I look back and know that's not the case but they made me doubt it. (Karen, IT)

Likewise, Sammy described how her boyfriend would act differently around their friends and family, to the point where she believed that it was, perhaps, okay that he made her feel like he did because he could be so nice when he wanted to be and perhaps he was just trying to help her "become a good girlfriend". She was 
particularly vulnerable to his controlling tactics as this was her first serious romantic relationship.

Others saw it [our relationship] as perfect, like "he cooks and cleans" and "he's so sweet how he protects her". I guess it did look like we were in a movie where he did so much and seemed so manly - caring and doing everything for me, but actually it was boring. It meant that I couldn't be me, I couldn't think for myself or survive on my own. He set up a dependency thing on him because he was in control of everything. I couldn't decide what to spend my own money on, I had to check what I was allowed to do when I left the house, he even checked any of my Facebook updates before I was allowed to post them. Through me, he created this front to my mates who thought I was happy, who thought he was just such a great guy. My mum even told me he was a good catch and not to mess it up, how could I leave after that? (Sammy, IT)

These young men's ability to modify their behaviour in public meant that those outside of the relationship interpreted 'kind' gestures as such, when actually these behaviours were experienced as tactics to evoke further control and terror. Adams et al. (1995) describe how such a scenario can be interpreted by outsiders as a loving warm gesture, while the behaviour evoked fear and terror for the victim:

The ambiguity serves both to increase the woman's sense of isolation and to limit her ability to respond by camouflaging to outside observers the threatening message. (p. 392)

Laura described this scenario when her boyfriend showed up unannounced with flowers to her friend's house. Her friends interpreted this behaviour as romantic and sweet but it demonstrated to Laura that her boyfriend had the ability to find her wherever she was.

We'd had a massive argument; I was quite scared so I just took off to my friend's flat. I knew my girlfriends would be there and a couple of their flatties. Safety in numbers, right? It wasn't long after I got there that Harry turned up. I freaked, how did he know I was there? How did he find me? He walked in nice as, he had flowers and a bottle of wine for my friends and sat 
down with us as if he had been invited to hang out. My friends had no idea that we'd had an argument and how scared I was of him. And they all thought he was so cool, such an amazing boyfriend. I just played along. I didn't want them to know the truth, and I didn't want him to flip out even more when we got home. I just had to play happy girlfriend and hope he wasn't mad as hell when we got home. (Laura, IT)

While participants' narratives classified as 'intimate terrorism victims' centred around modifying their behaviours to comply with their boyfriends' expectations, they also described the constant change of expectations and rules from their boyfriend which created an additional level of fear for them where they were never sure what was expected of them and what might lead to their boyfriend's anger/violence. New Zealand researchers Alison Towns and Hazel Scott (2008) described this use of everchanging rules as a way to keep women in a constant state of uncertainty and how this allowed young men greater control over what young women do and how they act. By changing the rules on what was acceptable behaviour and what was punishable, the perpetrator creates a sense of additional control, or feeling of no control for the female, by creating more fear and uncertainty for them.

Laura described her experience of her boyfriend's ever-changing rules and how she would do her best to abide by her boyfriend's rules to avoid conflict and violence but these seemed to change; in situations where she had once been okay, now he seemed to snap.

[What motivated his behaviours?]. I'm actually not sure, I mean I tried so hard to do what he wanted but I just couldn't get it right. I could never figure out what the rules were or what would set them off, I just didn't know when he would snap or what would set him off. (Laura, IT)

Not only were the rules ever-changing and the consequences of breaking the rules inconsistent, there was also a double standard in how these rules were applied. Similar to Towns and Scott's (2008) findings, male participants in this study described how the rules were only applicable to their girlfriend and not themselves. The young women had no control over what the rules were, or what was acceptable behaviour for her or him. This became a powerful tool in the range of controlling tactics that 
intimate terrorist perpetrators employed as it left the victims feeling disempowered and frightened of their partners' possible responses to their behaviour. Sarah described how there were definite rules for her with varying consequences if she broke them, but the same rules never applied to her boyfriend.

He could go out whenever he wanted with other girls and drink and party all night. Then he would turn up at my place at 3 am expecting me to have sex with him. He would go out all the time and I wouldn't know where he was. He could do what he wanted. I wouldn't even ask to go out without him, and if I did do something, like with my family, I would let him know what I was doing throughout the night and make sure I got back to his place when I said I would be there. And then hope he was in a good mood and that I hadn't gotten anything wrong. It really wasn't worth going out in the end, there was too much stress about what he was stewing about at home and what state he would be in when I got there. (Sarah, SCV)

\section{USE OF THREATS AND MANIPULATIVE LANGUAGE}

Another example of control through non-violent tactics was the use of manipulative language, which was commonly described by the young women and men in this study, and often in the context of keeping the relationship together. This was supported by Bay-Cheng and Goodkind (2016) who reported the need young women felt to be in a relationship as a result of the dominant narrative that defined successful adulthood as being in a romantic relationship. Towns and Scott (2008, p 75) extend this narrative from their work on the Culture of Cool by concluding that "for some young women within this culture it is cool to be a girlfriend: being one provides a girl with social standing and allows her to be popular".

For example, Mary described how she used threats of self-harm when her boyfriend broke up with her.

My boyfriend broke up with me just after my birthday. I had told him if he ever broke up with me I'd kill myself. I went through severe depressive states. I had bi-polar/depression. I knew he wanted to leave so I was in this real suicidal phase. I think it kept him around a bit longer but then he just had enough. It was the same with my mum, she didn't know what to do with me and so she 
kicked me out of home for acting out, and screaming. I guess I needed help but people just chose to leave instead. (Mary, SCV)

Jack described a similar situation where he had tried to break off a relationship, but the female threatened to self-harm, forcing him to remain in the relationship to avoid her following through with her threats. Jack described an incident where he ended the relationship and his ex-girlfriend was hospitalised. After that they got back together for a while, but the relationship eventually broke down and he ended the relationship soon after, with the same threats of self-harm occurring again.

She said stuff that was worrying - I called the police because I was real concerned she'd kill herself. We broke up a few times, she always rang saying "I love you, don't leave me, I can't live without you, I'd rather die", I just couldn't handle her crying, I guess I went back even though I knew it was the wrong decision, I didn't want her to be distraught. And I definitely didn't want her death on my hands. When you care about someone it can be to the detriment of each other especially when the relationship isn't healthy. It was pretty messy for the next few months and it still ended bad, staying longer didn't help. (Jack, SCV)

The potential of the relationship ending was a very strong motive for some participants to try and control a partner's behaviour. This was a particularly powerful narrative for females who felt a strong social pressure to be in a relationship to maintain social standing. As discussed in Chapter Seven, female participants described the social pressure to be in a relationship even if it was a negative relationship, as well as the need to be in a relationship to gain self-worth and to legitimise sexual relations.

Rebecca provided her experience of this concept where she worked to maintain a relationship that she was not happy in, in an effort to avoid becoming single.

He said he was going to leave me; I couldn't let that happen, I couldn't be single. It was better to be with him and try and avoid arguments than have to be single again, to have to be back out looking for a guy. So I just did what he wanted me to. (Rebecca, SCV) 
For male participants, relationship dissolution was also problematic but for a different reason. Male participants presented a narrative that demonstrated that they could lose status amongst their peers if it was their partner who ended the relationship. Therefore male participants described how they would try and "get in first" and end the relationship themselves, or as Towns (2009) states "control the ending". Towns found a similar theme in her New Zealand study on the Culture of Cool and Being a Man where men ended the relationship to avoid humiliation and maintain their pride. This need to control the ending of the relationship was demonstrated by Joe where, through his narrative, he described how he assessed the possibility of being 'dumped' by his girlfriend as being too risky for his reputation and that it was optimal for him to end the relationship before his girlfriend, as an attempt to maintain his social standing with his peers.

She was talking like she had had enough; she said she was ready to get out of it [end the relationship], like I couldn't be the one who got dumped. That would be so lame; you can't tell your mates you got dumped so I had to end it first. I guess I called her out. She was threatening to break up with me, ah, no, I don't think so bitch. I liked her but I wasn't going to change for her, she'd still probably be unhappy, girls just are never happy, it's never good enough. So see ya later! I win. (Joe, SCV)

Another tactic, and one that was described across a majority of the interviews was the use of and presence of emotional abuse across the relationships through different behaviours. Emotional abuse is a prevalent form of aggression that consists of a range of tactics that emotionally target the victim with the intention to control, demean or threaten their partner. Such behaviours include put downs, making them feel bad about themselves, calling them names, making them think they are crazy, playing mind games, humiliating them, and making them feel guilty. These behaviours can result in the partner becoming isolated, restricted in what they are allowed to do, humiliated or degraded (Hammock, Richardson, Lamm, Taylor, \& Verlaque, 2017).

There was a very strong narrative about the use of emotional tactics by all the participants, both males and females and in situational couple violence and intimate terrorism relationships. Common amongst all relationships, for participants and/or 
their partners, was the use of emotional tactics such as name calling, shaming on social media, and comments to make the person feel worthless.

He called me horrible names, it would start on something small, then get big, he'd leave, then eventually come back and apologise. It was petty stuff that didn't really hurt. He called me horrible names but I did deserve it for what I did [cheated on him]. We just didn't deal with the downs; we swept them under the carpet. Eventually there was no room under the rug. It just had to come out. I guess the only way for him to get the frustration out was to be honest and brutal about what he thought of me. I was a stupid slapper and I was a worthless whore. He was just being honest. I deserved that too. (Kathryn, SCV)

The difference was that within the situational couple violence relationship the emotional abuse experienced by participants was not embedded in the same levels of control and lacked the 'coercive' elements described by participants in intimate terrorism couples. Within the intimate terrorist relationships, the emotional abuse the female participants described was as part of a pattern of control and appeared to have more negative effects, especially when young women described their isolation through their boyfriend's behaviours. Whereas seemingly less severe tactics such as name calling and put downs were harder for these female participants to validate as lies when other support was not there to dismiss the claims.

The significant impact of emotional abuse has been documented as possibly worse than physical violence with greater negative outcomes and damage from constant attacks on self-esteem and self-worth (Anderson, 2008). This was evident in Karen's interview where she described the terror of the physical violence she experienced inside an intimate terrorism relationship but also how it was intertwined with psychological elements.

Physical is scary at the time because it's happening, it hurts, but the mental is far worse, I was always thinking where is he? What kind of mood is he in? What have I done wrong today? What next? He was just so unpredictable. There was plenty of physical stuff but the manipulation was all the time. The physical rage comes and goes but the mental shit is ongoing. Occasionally he 
would get physical as a way to freak me out, but I was more terrified of what was going on in my own head, thinking about what he was capable of, what he might do. How bad it would be this time if I didn't do what he told me. (Karen, IT)

Another non-violent tactic is economic abuse. In this study economic abuse in dating relationships was not a predominant feature of participants' narratives. Behaviours described in the IPV literature include controlling access to money, prohibiting the partner from having a job, earning an income or having access to their income (Pence \& Paymar, 1993). The absence of economic abuse by participants in this study may be a by-product of the nature of dating relationships set in the current New Zealand culture of hooking-up and the age of participants. There were few couples in this study who had combined finances, or they may have had a joint account for the shared dwelling but still had primary control over their own finances.

However, there were two examples of participants in more longer-term relationships where economic abuse was described; one was in the context of a situational couple violence female having more power financially and the other was within an intimate terrorism relationship where the boyfriend took money from their business before leaving her with the debt he created.

Emma described how her boyfriend used particular financial tactics to control their relationship and her independence. Her boyfriend was unemployed while she had a fulltime job. Emma described how, although it looked like she had the financial independence and control, he actually took that away from her, in what could be framed as a display of hegemonic masculinity reflecting his need to regain a perceived lack of control and status by not being the financial provider in the relationship. Since he was not able to be in control of the financial side of the relationship because he had no income, he created control by restricting their activities. He also employed violent tactics if she suggested they go out, as her 'shout'. As a rule they did not go out in public as that would upset the gendered power balance, although he would 'allow' her to spend money on private activities like PlayStation games and expensive food and alcohol. 
We don't go out much because he's got no money and he says that the man has to pay. So we just don't go out. I mean, nah, he would never let me pay. He totally thinks that he should pay. After a few offers to shout him a night out he started to get really shitty. One night he just lost it. He said I was rude thinking it was acceptable that a chick pays for a dude. He slapped me on the face real hard, so I guess I learnt from then not to bring it up again. I mean we just ended up hanging at home playing PlayStation and eating takeaways. He didn't mind if I spent money on that stuff, maybe because it wasn't in public, and it was his decision what to buy so I guess he had the upper hand on that one. (Emma, SCV)

Within the narrative of the intimate terrorism relationship, economic abuse appeared significant and ongoing and endured past the end of the relationship. Karen described how she and her boyfriend went into business together. However, he took control of the business aspects of running the restaurant while she ran the staff and floor. He made these roles clearly distinct and, as a result, he would prevent her from having access to the business financial accounts. As it transpired, when the relationship ended Karen found out that he had been taking money from the business and had incurred huge debt which was all in her name as he had a criminal history and had convinced her that the business needed to be in her name.

This section has detailed the range of non-physical tactics that participants talked about throughout their interviews, the context in which these tactics were used and how this interacts with Johnson's typology. The next section presents the experience of physical and sexual abuse in dating relationships described by participants.

\section{SECTION TWO: PARTICIPANTS' EXPERIENCES OF PHYSICAL AND SEXUAL VIOLENCE}

The previous sections described the non-physical tactics that were reported by participants in this study, alongside these narratives were reports of physical and sexual violence. These tactics can be used to enforce, enact or demonstrate an intimate partner's control. If the non-violent tactics fail to control their partner or the partner is seen to fight back, then physical tactics can be used to regain power and control, assert strength and prove intention and capacity to harm (Stark, 2006). 
The physical tactics experienced by participants in this study ranged from pushing, shoving, slapping, punching through to being repeatedly hit, kicked and punched, and in three instances a weapon was used to cause injury.

Participants within their dating relationships commonly experienced minor acts of physical violence such as pushing, shoving and slapping. These behaviours are categorised in the CTS2 as minor acts, a categorisation that complements participant narratives that it was "just a wee push" or "I just slapped him".

I just kinda slapped him, like in the movies. Chicks do it all the time. It isn't a big deal, I was just doing it to make a point, to get his attention. (Mary, SCV)

A large number of these minor acts of violence were described within mutually violent situational couple violence relationships and were often seen to be mirrored. For example, one partner would push or shove, so the other partner would do this back, almost as a way to mimic their partner so as to regain power balance or due to a lack of coping techniques.

There was pushing and slaps in the relationship, I didn't hit back, but I did push back. Once he pushed me up against a wall so I shoved him back really hard. I don't think he expected it because he totally lost his balance. (Beth, $S C V)$

[Was there violence in the relationship?] Any violence? nah, oh, I don't know. I mean we would yell quite hectically at each other and call each other horrible names. It would start with a little problem then I'd explode and he'd explode and it would end up as a yelling match where you took a stab at every little thing you had, any ammo that you could think of. (Rebecca, SCV)

For Sarah, biting was a tactic she described using frequently, especially when her boyfriend was holding her down or trying to stop her from hitting him. This was also where Sarah described 'mutual' violence, where he would physically break objects and hit the wall next to her head. Note here that while there was mutual violence it was not equal in terms of the described impacts, with Sarah describing the initiation of violence by her boyfriend, the physical injury caused by her boyfriend and the 
greater level of fear from his violence as he was far more dominating and physically stronger.

Sarah - I guess you could say that I harmed him, I would just bite him because I was so bloody angry, I just wanted to hurt him. He was often close enough to bite because he would hold me so I would listen to him, so I couldn't escape when he was trying to tell me something. So I would bite him.

Interviewer - what would his reaction be to that?

Sarah - He would bang the walls, like smash the wall next to my head like he was gonna hit me but he didn't, maybe if he was really shitty he might've. It scared me because I didn't wanna get hit and I didn't want him to get hurt when he smashed things. (Sarah, SCV)

What was obvious from the interviews was the prevalence of these more minor acts of violence amongst those in situational couple violence relationships, and that for the majority of these situational couple violence relationships this was the extent of their experience of physical force. However, for intimate terrorism participants, while they did describe many of these minor acts, they were much more likely to be part of pattern of more severe physical force.

The more severe end of the CTS2 has items such as "I punched or hit my partner with something that could hurt". While these acts were not exclusively described within the context of intimate terrorism relationships, there was little mention of this more severe violence by situational couple violence participants. The goal of using more severe physical violence by intimate terrorist perpetrators was described by participants as a way to enforce threats where intimate terrorism physical violence was part of a range of other tactics used by the perpetrator to hold their partner captive, prove their physical power and ability to dominate.

The most extreme example of physical violence in this study was the recounting of an incident by Regan, a young man classified as intimate terrorist, where he described strangling his partner. A strong risk factor of IPV homicide is the use of non-fatal strangling by an intimate partner (Glass et al., 2008). Here Regan described how this incident unfolded and the harm it caused his girlfriend. 
Just this one day, she was just like, "nah nah nah”, she wouldn't shut up. I started yelling at her to, "shut the fuck up". The next thing I know I had my hands around her throat. For a second I was like, "yeah she's bloody quiet now" then she started to go a bit limp, like at first she was fighting back, but then she just didn't seem to have the strength. I freaked a bit and let go. It just happened so quick. It was pretty freaky, I guess we both saw how strong I could be and what I was capable of. (Regan, IT)

The use of choking and strangulation by intimate partners was highlighted in the wellpublicised 2018 murder of Grace Millane. On the 8th of December 2018, 22-year-old British backpacker Grace Millane, who was travelling in New Zealand, was murdered by a 26-year-old man while on a date. They had met through Tinder, a dating application, and subsequently spent the evening together drinking before returning to a central Auckland hotel where the man was staying, and where he killed Grace. Grace's body was then disposed of by the man who buried her in a suitcase, which he abandoned in local mountain ranges. The defendant was subsequently convicted of murder by a jury after just five hours of deliberation and on the $21^{\text {st }}$ of February 2020 was sentenced to life in prison.

Grace's post-mortem showed that she died as a result of pressure to the neck, highlighting the importance of recognising the severe consequences arising from such physical violence. This is reinforced by studies such as Glass et al. (2008) who report that non-fatal strangulation is a strong risk factor for IPV, and is strongly associated with attempted or completed homicide.

In New Zealand, the growing awareness and seriousness of strangulation in intimate relationships has recently been recognised with a new offence for the strangulation or suffocation of someone. This new offence came into effect in December 2018 under New Zealand's 1961 Crimes Act and carries a maximum sentence of seven years imprisonment. This new offence was recommended by the New Zealand Law Commission after their 2016 report concluded that strangulation by an intimate partner increased the risk of fatal attacks for victims (Te Aka Matua o te Ture Law Commission, 2019). Since the law came into effect on 3 December 2018, through to 28 February 2019, 416 people had been charged by the New Zealand police for 
strangling or suffocating their intimate partner (Te Aka Matua o te Ture Law Commission, 2019).

\section{WEAPONS TO CAUSE INJURY}

While the use of physical violence was common across all participants, due to that being part of the criteria for inclusion in the interviews, the use of a weapon to enact violence was less frequently described by participants. Of the 16 participants, five used an object or weapon against their partner. In these scenarios the object was often within reach and used opportunistically in the heat of an argument, rather than being a pre-meditated or intentional action. Here Jack describes causing injury with an object, in the context of situational couple violence.

Jack - She told me I was being psycho. She went to grab her phone so I grabbed it first and threw it at her.

Interviewer - How did she react?

Jack - It left a whopping mark on her forehead, she was a bit shocked I guess. I was real sorry straight away. I don't really know what came over me, it wasn't like me, I just saw her go for it and I got in first. (Jack, SCV)

One of the three participants who reported using an object to cause harm was Sarah, a young woman who was recorded as situational couple violence as was her partner. In this excerpt Sarah describes how she used a wine bottle to make up for her lack of size and strength.

We were arguing again, sometimes it felt like a bit of a game, I'd win if it was using my head, I could out smart and out talk him, but when it got a bit physical he'd usually win, I'm just not as strong as him. This one time we were pushing each other and arguing and I decided it was enough, so I grabbed a wine bottle nearby and used it to hit him on the back. You know, make it a fair fight. It made him stop and I won that round. I don't think I hurt him and I'm pretty sure he could've grabbed the wine bottle off me and hurt me worse, but in that moment it was enough to shock him and for me to come out on top.

(Sarah, SCV) 
For two of the participants in intimate terrorism relationships, the use of a weapon was described as more intentional. For both Jessica's description of her boyfriend's violence and Ricky's description of his own violence, the use of a weapon came on the back of multiple threats to use violence and was seen as necessary by the perpetrator as other non-violent control tactics were not effective in eliciting power and control. Jessica described one episode that lead to her boyfriend using a knife on both her and himself.

It had been this ridiculous week, I think now I can see how he was really unravelling. I had tried to push back a bit, like defy him I guess. I really wanted to go to this party with my girlfriends and he wasn't gonna let me. And I told him I was going anyway. He grabbed me and dragged me through to the kitchen and grabbed a knife out of the block. I can still feel the fear, the feeling inside of, yeah, of fear. I thought "wow this is it, I'm gonna die over a stupid party". He was waving the knife around all like "I warned you not to cross me bitch". And he cut me across the arm, said my neck would be next. He was just going psycho, next thing he's cut his wrist flipping out saying "look what you made me do". That's when his flatmate walked in. You know he probably saved my life in that moment. He called an ambulance and the Police came. It was chaos. I could've been on the news as some poor girl who'd been killed by her psycho boyfriend. (Jessica, IT)

\section{SeXuAl COERCiOn, ViOLENCE AND RAPE}

This section has so far demonstrated the high, frequent and sometimes severe physical violence that was described by young adults in this study. Also described in this context were forms of sexual coercion, most often unwanted sexual activity. Like other studies such as Beres, Senn and McCaw (2014), a broad definition was applied to narratives of coercion. Here, sexual coercion covers a range of non-physical behaviours used to force someone to perform sexual acts and can include psychological acts, blackmail, threats and intimidation, and includes having sex with someone who is unable to consent, for example through intoxication (Conroy, Krishnakumar, \& Leone, 2015).

Three distinct types of sexual coercion have been conceptualised by Conroy et al. (2015): forced, coerced and willing unwanted sexual activity. Forced sexual activity is 
defined where physical force is used to 'obtain' sexual activity from an unwilling partner. Coerced sexual activity is where non-physical force is used to obtain sex, while the third category is engaging in willing unwanted sexual activity where a partner consents to sexual activity although they do not desire to engage in this (Muehlenhard \& Peterson, 2005). While this typology of sexual violence may have limitations, it provided a useful framework for the narratives of young people in this study.

Willing unwanted sexual activity was a commonly described experience of participants in this study, and most young adult females described situations where they consented to have sexual intercourse with their partner or as part of a hook-up, even though they did not necessarily desire sexual activity. This is consistent with other studies that report women are more likely to consent to this behaviour (O'Sullivan \& Allgeier, 1998). The most common narrative around willing unwanted sexual activity was one where they did not want to disappoint their partner or to cause an argument. Katz, Kuffel and Brown (2006) reported a similar description of young women's willing engagement in unwanted sex where they reported consenting to this activity to avoid feelings of tension in the relationship, to promote intimacy and to satisfy the needs of their boyfriends. Gavey (2019) also described how young women engage in casual sex for reasons devoid of their own desires but for reasons such as wanting to please their partner and to conform to compulsory heterosexuality. These were evident in the current study:

Sometimes you just have to sleep together, guys like sex and it keeps them happy. I often had sex out of duty. (Kathryn, SCV)

I went home with him, I knew that meant we'd likely have sex. I'd hoped we could just play around but then he wanted to go all the way so I felt like I had to, I went home with him so that pretty much implied I was up for it. I didn't want to have to deal with how he'd react if I said no. (Beth, SCV)

As mentioned above, Conroy et al's (2015) categories, used here as the framework for this analysis on sexual coercion and violence, have limitations, in this sense the oversimplification of a binary of willing and unwanted sexual activity. As Beres (2010 p.10) writes: 
... willingness to participate can, at times, be incongruous with sexual desires and that there are a myriad of reasons why someone may agree to have sex.

It is therefore important to acknowledge, as shown by the narratives in this section, that experiences and feelings around engaging in sexual activity are far too complex to capture in a binary of wanted or not, and of being willing or not. In other words, that wanting sex and consenting to sex are not the same, where sex can be consented to but not wanted or where sex may be wanted but consent is not given (Muehlenhard \& Peterson, 2005).

While willing unwanted sexual activity was common in this study, so was coerced sexual activity. Like other studies reporting on coerced sexual activity (see Katz et al., 2006), participants in this study commonly described verbal pressure in the absence of physical force. This was described by the young women in the study as a common experience. There were three scenarios that came out of the interviews with these young women; the first being the use of threats or emotional manipulation by their partner to coerce them into sexual activity.

There were times where I really didn't want to sleep with him. I just didn't want to. But he would just keep pushing it and say that I owed him for taking me out for dinner or something. In the end I felt like I had to have sex with him just shut him up. (Emma, SCV)

This quote challenges the theory that sexual violence and coercion is a result of miscommunication of consent between young men and women. The Miscommunication hypothesis posits that men misunderstand or misperceive refusal messages to their sexual advances (McCaw \& Senn, 1998). However, this quote from Emma and other narratives in this study indicate that young men deliberately persisted with sexual advances even when a young woman indicated she was not interested. Even when refusal to young men's sexual advances were displayed as a physical response, and not verbalised, young men ignored these signs. The persistence of young men to engage or progress in sexual activity when they faced resistance is a factor associated with increased risk of sexual assault for young women (Beres et al., 2014). The use of manipulative tactics is also described through Emma's quote above - as a response to her resistance, her boyfriend used guilt tactics to manipulate her 
into consenting to having sex with him, which is a theme reported across other studies of coercion such as Littleton and Axsom (2003).

The second scenario of coerced sexual activity, described by most female participants as deeply entrenched in the hooking-up culture, was where the over-consumption of alcohol resulted in participants being coerced into sexual activity. This type of alcohol-fuelled coerced sex was 'normalised' by participants who described socialising with friends, getting drunk and then ending up going home and sleeping with someone, often in an intoxicated state. In this sense the experience of coerced sexual activity was embedded in what Fisher, Daigle and Cullen (2010) described as the routine activities and lifestyles of young adults as they engaged in the night-time economy and drinking to intoxication. This type of coerced sexual activity was described exclusively by female participants, at the hands of a male. The alcoholfuelled situation led to young women feeling confused about their level of consent and knowledge of what happened due to memory loss from their intoxication.

It's not that great probably; I've been out a couple of times and gone home with random guys. That's how me and Simon hooked up. I was off my face and we ended up back at his house. I kinda woke up during the night in this strange bed naked; I guess we'd had sex. Not that I can remember. It's not that great, I guess, I mean I don't really remember what happened, he coulda done whatever and I didn't even know. It's probably really not that cool, as a female it's probably really silly. He could've been a total psycho and I didn't know where I was or what was happening. (Sarah, SCV)

The third scenario was coerced sexual activity within a male discourse of young women pretending they did not want sex, but the male continuing. Some male participants rationalised that the young women's apparent resistance to sex was because they did not want to appear too easy, and it allowed the young man to be the dominant player in the scenario.

You know, when you're fooling around, sometimes she'd say "stop it" but if I just kept going a little bit more, made her really enjoy it, then she'd usually give in. Especially after we'd been together a while, it was harder for her to say no, like walk away. She never got real upset, if she'd gotten real angry or 
upset I would've stopped but she was more like "don't, come on, stop" and kinda giggly, so I reckon that's a good sign they don't really mean just stop. Girls do have to make sure they aren't being too easy, like giving it up too easy so that giggly flirty "no" is more them keeping up that appearance of being respectable, kinda a 'nice' girl. (Jack, SCV)

She led me on, I mean she instigated the kissing and so I took it from there. That's what guys do, they have to take control of the situation and be the man and direct the girl. It stops her from being, from looking like a slut, if she's too forward. Guys don't want that, they need to be more in control of it. Girls like that too, to be shown who's the boss. So I didn't stop. (Regan, IT)

This narrative was also evident in Hird and Jackson's (2001) research where they found that young men talked about girls fulfilling a role as the "gatekeeper", relying on young women to self-monitor and "close the gate" when they assess it is necessary.

Such positioning places girls in a no-win situation. They can deny their sexuality in order to conform to expectations of 'angelic purity', or girls can be sexual and risk being labelled 'slut'. Consistent with the 'angel' identity, girls are the gatekeepers of male sexuality (Bateman, 1991). The dichotomized construction of female sexuality as 'slut' or 'angel' creates conflict for girls as they negotiate their sexual identities. As 'sluts', girls are expected to sexually excite boyfriends, but as 'angels' they are expected to apply the brakes to rampant male sexual desire. One position this creates for girls is, in the words of a New Zealand girl, 'cock-tease'... Not surprisingly, this belief is often cited as a justification for rape. (p. 34)

While willing unwanted sexual activity was relatively common, along with examples of coerced sexual activity, forced sexual activity was less commonly described by participants and when it was, occurred within the category of intimate terrorist relationships.

Within the narratives of forced sexual activity was a scenario where the female initially consented to sexual activity but during the course of the activity the act became violent. In this context the sexual activity began with elements of 
sadomasochism (S\&M: the giving or receiving pleasure from acts involving the receipt or infliction of pain or humiliation (Weierstall \& Giebel, 2017)) but this soon got out of control and although the woman wanted the role-playing to end, her partner did not stop.

This raises a question around the construction of consent and whether viewing consent as a discrete event is useful, versus consent as a process that continues throughout the event of sexual initiation and throughout sexual activity (Beres, 2014b). Researchers on the subject of consent, such as Humphreys and Herold (2007), report that young men are more likely to view consent as being a discrete one-off event compared to young women who view it as a process that continues throughout the sexual event and across sexual encounters. In other words, consent was something young women saw as being an ongoing decision, not just in the first sexual encounter and at the start of the advances, but throughout the sexual interaction and for any subsequent sexual activity.

This construction of consent is described in Karen's account of sexual violence that occurred in the context of consensual S\&M activity, which turned physically forceful and caused her great distress and injury. She described how she enjoyed sex and consented to having sex with her boyfriend but on several occasions he became very physically forceful with her, turning her enjoyment of sex into something she began to fear. She described the resentment that grew towards her boyfriend and the shame she felt for "letting him do that to her".

I am a girl who enjoys sex. It's something lots of chicks enjoy. So of course me and Craig had sex, a lot. That's kind of how the relationship began, you know hooking-up one night, the sex was great back then. But after a few months he started to get real nasty. It started as a little bit rough, like slapping me on the arse and role playing, talking dirty; which I thought was really hot, very '50 shades of grey'. But then he like ramped it up and started slapping me across the face, he started to push real hard, I mean like you know, it's embarrassing but like thrusting real hard until I bled, and I was crying, begging him to stop, waiting for it to be over. It didn't take long from then to really hate him, to hate having to sleep with him. I look back now and can't believe I let him do that to me so many times, it's pretty shameful. (Karen, IT) 
Another narrative around sexual coercion was in the context of the use of media and technology and its role in facilitating manipulation of the victim. Two participants described acts of consensual and enjoyable sex within the realms of a normal 'healthy' relationship. However, both described the after-effects of discovering that the sexual activity had been recorded without their consent and the later use of those recordings by their boyfriends to control and manipulate them.

Like forced sexual activity, this type of manipulation was perpetrated by male partners, and in the context of intimate terrorism relationships as part of a wide range of tactics used to enforce power and control in the relationship. Sammy described how her boyfriend used a recording of them having sex to keep her in the relationship, with her boyfriend threatening to post the recorded clip if she left him.

I didn't know he was doing that, that he was taping us having sex. It became something he used all the time if I said I was going to leave, he would threaten to post it to the web, once something's on the web you can't get it back. I would freak out about who might see it, maybe I wouldn't get a job, I mean it was totally embarrassing seeing myself like that, totally degrading. It was utter betrayal. (Sammy, IT)

The second participant described a similar situation where a video was used as a threat to keep him in the relationship, but in this instance the underlying threat was that the video would be used to 'out' him. Grant described how this was his first homosexual relationship and his boyfriend used the fact that Grant had not told his family or friends that he was gay as a way to control him and keep him in the relationship. The physical evidence of the video made the situation more horrifying for Grant as it was not only something he did not want to have out in the public arena but it was also concrete proof of his relationship with another man.

It was the worst moment of my life. To be threatened, that if I didn't stay he would out me through some sex video that he had of us. I was horrified. I felt really powerless to do anything. One press of a button and the video could be seen by everyone. I wasn't ready to let the world know I was gay, and even if I was, I didn't want something like that in the public domain. It was a 
nightmare. At that moment all I felt like I could do was agree to stay, to make him think I loved him and wasn't going anywhere. (Grant, SCV)

All participants experienced minor acts of violence, however more severe violence was most likely experienced in intimate terrorist relationships. Unwanted sexual activity was commonly described, and in somewhat normative ways, where young women consented to sexual activity even though they did not desire to engage in it. Coerced sexual activity was less likely to be described by participants, and when it was it was often in the contemporary social context of hooking-up and drinking where alcohol played a role in participants' experiences.

\section{CHAPTER SUMMARY}

This chapter has described the range of non-violent and violent tactics experienced by participants within the context of their dating relationships and provides positive support for Johnson's (2008) typology in showing that there are difference between the types of violence experienced in young adults dating relationships. In support of Johnson findings (see Johnson, 2009a; Johnson \& Leone, 2005), this chapter found that situational couple violence couples were more likely to report more mutually perpetrated, lower impact violence that resulted from conflict that escalated to violence. In contrast the pattern for intimate terrorism was more likely to be unidirectional violence where young men were the perpetrators and this type of violence was more likely to result in more negative impacts for young women including greater levels of psychological harm and physical injury.

Alongside the common experiences of dating violence for participants, was the persistence of technology and social media in the monitoring and surveillance of dating partners where these became tools that enabled participants to monitor and control their dating partners (Baker \& Carreno, 2016) and extended the ability for dating violence to occur (Flood, 2019).

The next chapter will provide a detailed analysis on the attitudes and beliefs presented from the narratives by young adults throughout the qualitative interviews. A significant body of research has demonstrated that holding certain attitudes and beliefs can create a culture where some violent acts are condoned, which provides an environment that supports justification, minimisation and excuses for such violence 
(Harris, Honey, Webster, Diemer, \& Politoff, 2015; Nabros \& Jasinski, 2009). Given the attitudes and beliefs that were presented by young adult participants, and their impact on the nature and experiences of dating violence, an examination warrants its own chapter. 


\title{
Chapter Nine - AtTitudes AND Beliefs AND THE CONTEXT OF DATING VIOLENCE
}

\author{
...we need to address the underlying gender norms that shape the way this \\ violence is understood and allow for the continued perpetration of such \\ violence. In other words, we need to provide alternative norms about both \\ masculinity and femininity that value non-violence and non-aggression in boys \\ while allowing for vulnerability. (Beres, 2017, p. 7)
}

The previous chapter described young adults' experiences of non-physical and physical acts of dating violence. This chapter provides narratives on the context within which this violence occurred and the way these experiences were minimised and excused.

I have focused this chapter on the interview data that adds to the significant body of research on the role of beliefs and attitudes that support violence (Nabros \& Jasinski, 2009). Holding attitudes and beliefs that support violence does not necessarily mean that someone will use violence, but evidence suggests that when such attitudes are present across a group of people this can create a culture where some violent acts are condoned. This in turn can create a culture that supports the justification, minimisation and excuses for such violence (Harris et al., 2015). These attitudes and beliefs not only impact on the perpetration of violence, but also on victims' responses to such acts and the way that communities holding such beliefs respond to this violence (Flood \& Pease, 2009).

\section{SECTION ONE: MINIMISING AND JUSTIFYING DATING VIOLENCE}

In the current study participants' narratives centred on minimising and justifying the violent behaviour both from the way that victims described and made sense of their experience of violence and in the way that perpetrators described their use of violence. This section is written in a more explicitly gendered way as the analysis is largely situated within a discourse that supports traditional gender norms and beliefs. Therefore I use terms such as female victims and male perpetrator to describe how participants justified and minimised violence in their dating relationships. 


\section{VICTIMS' JUSTIFICATION OF THEIR PARTNER'S USE OF VIOLENCE}

One way that female participants justified their experience of control and violence was to minimise it by defining behaviours and tactics as normal and okay. Some female participants minimised the violence because they did not believe the behaviour was wrong, while others created excuses to hide or ignore the violence. The common narrative pertaining to the minimisation of violence was the portrayal of the partner as 'not normally violent' and that the relationship was 'generally fine'. Karen and Sue articulated this when they described their partners' frequent use of violence.

He doesn't realise what he is doing to me. It's almost worth putting up with because when he's lovely, he's an awesome guy who can cook well and is generous with money and time. I guess he flips between being a gentleman and a monster. When he's nice he is so wonderful, I guess that's what made me stick around longer. (Karen, IT)

He would change things long enough, he would change enough for a couple of days and then I'd be like "oh, maybe he is ok and he can change, it could be worth staying". And then he would slowly move back into his old ways and you'd still think he is a nice guy, I mean I know he has it in him to not be like this. (Sue, SCV)

Another way participants minimised controlling behaviour was through their narratives around the perpetrator in the role of protector, where his behaviour was reinterpreted as a "knight in shining armour" and his behaviour was a sign of love and caring, not of jealousy and control (Chung, 2005). Although participants in controlling and violent relationships felt trapped, scared and powerless, Sammy explained how her boyfriend's motives were based on him wanting to be a nice guy and look out for her.

Well, it was just that he really cared about me and wanted to make sure I was ok, that he was just making sure I was with the right people, that I was not out being slutty, or not getting drunk and looking silly. (Sammy, IT)

Within this narrative of minimisation was a sub-narrative of violence tolerance. In this sense, the violence was "not that bad", suggesting that the violence was somehow tolerable since it was not as bad as it might have been. Sammy described how her 
boyfriend's violence was tolerable as it was not nearly as bad as the violence she experienced in childhood, demonstrating the normalisation of such behaviour when it is part of a child's experience.

My first boyfriend was very placid, fights wouldn't get too violent. Tim's more verbal, he doesn't mean it but makes me feel stink, I don't class that as abusive, just a throwaway line I wasn't supposed to go away and analyse. I guess when he did get violent and stuff it was scary, I guess that's when I saw that he's very like my father, when it's good it's really good, but when it's bad it's really shit. But the bad times weren't so bad, not as bad as Dad was so it was easier to hang around for the good times. (Sammy, IT)

Violence was also minimised through placing the behaviours in the context of play. As reported in the literature, violent incidents were reinterpreted by participants in this study as 'play fighting' (Foshee V., Bauman, Linder, Rice, \& Wilcher, 2007). This was most commonly reported by some participants in situational couple violence relationships, where violence was framed as a "joke" or "just playing around". Even when injury occurred, this was described as "over-zealous play" and "not intended to cause harm". However, the impact from play violence and demeaning and derogatory jokes often resulted in negative outcomes.

It was just play fighting, it wasn't aggressive, we'd just push each other. A few times one of us would end up with bruises but nothing major, it was just when it got out of hand. If he was ever actually violent I'd leave him. But he's not, really. (Sarah, SCV)

Emma described incidents where she was injured as a result of 'play' but she found it hard to link her label of the behaviour as play with feelings of not being okay about the situation where her boyfriend caused harm and injury.

Interviewer - How did you feel during or after this happened, after the play fights ended?

Emma - It wasn't that bad, I knew it was just mucking around. I guess when it happened I was a bit worried about whether he would stop, and when he 
started to hurt me I felt a bit scared ... but as soon as he saw me cry he stopped and apologised.

Interviewer - Oh, so he stopped as soon as he realised it started hurting, did it happen again?

Emma - Well he stopped eventually, I guess he might have thought I was over reacting, but after a couple of minutes he must've seen how freaked out I was getting, it had happened before, well lots of times. But he did always apologise. (Emma, SCV)

Two other female participants described how the joking and derogatory comments about them impacted significantly on their self-esteem and how put-downs, in the context of "joking around", affected their self-image. Jessica described how her partner would joke about her appearance and say he did not want to have sex with her "because she was a fatty". Jessica described in detail how his joking behaviour made her withdraw into the relationship where she felt increasingly trapped by other controlling and harming behaviours that continued to build as her power decreased. Jessica was left feeling worthless and with little strength to leave the relationship.

He did it as a joke, he would laugh at me and call me names, but it made me feel stink. Often it would start with sex, he would tell me to "get my fat arse over here" then say, "I figure I owed you a couple [have sex] since you are my girlfriend, even though you're a fatty." "Maybe I'd give it to you more if you lost a few kilos." Then he would laugh and say "oh, don't get upset you know it's just jokes", but I don't think it was. And it didn't make me feel good. I just started to hate how l looked, I even ended up starving myself for a while. Then he made fun of me for puking [vomiting] all the time. I just had to start being more secretive about it all. I just wanted him to say I was pretty. I'd ditch class and exercise for hours. Then I failed a class, he said I was so stupid, “you can't even pass a simple undergrad course”. He laughed, he thought he was hilarious. It was true, though, I was stupid. You know, it wasn't long after that I dropped out of Uni completely. It suited him because I just worked and earned him money and I could do more for him and do what he needed around the flat. (Jessica, IT) 


\title{
Perpetrators' JUSTIFICATION OF Their USE OF VIOLENCE
}

Perpetrators in this study frequently justified their own use of violence against their dating partner. Attitudes that men's violence is justified and acceptable are shaped by socially constructed gender norms and constructions of masculinity such as that men are biologically more prone to violence, it's harder for men to control their emotions, and that men have strong natural sexual urges and desires (Brown et al., 2010; Butler, 1994). These beliefs extend to blaming women for their role as a victim because they deserve it or provoked it, for example if they dress in a provocative way.

\begin{abstract}
Attitudes have a fundamental and causal relationship in the perpetration of violence against women. There is consistent evidence of an association between violence-supportive beliefs and values and the perpetration of violent behaviour, at both individual and community levels. For example, men with more traditional, rigid, and misogynistic gender-role attitudes are more likely to practice marital violence (Heise, 1998; O’Neil \& Harway, 1997). Boys and young men who endorse more rape-supportive beliefs are also more likely to have been sexually coercive (Anderson, Simpson- Taylor, \& Hermann, 2004). (Flood \& Pease, 2009, p. 126)
\end{abstract}

There was a strong narrative by the two intimate terrorist male respondents around blaming their girlfriends for their need to use violence. Regan's excuse for his violence was his girlfriend's failure to act the way he wanted, so if she did not play the role of the perfect girlfriend and follow his authority then he had the right to punish her as a way to enact and regain control and intimidate her.

Most of the time it was when she wouldn't listen, when she didn't do what I told her to, she knew I had strict rules and expectations, that was part of us being together, she knew what I was like. So if she didn't do the right thing then I had to punish her, to let her know that wasn't ok. She knew what she needed to do, so if she broke the rules then the consequences were on her. (Regan, IT)

For Ricky, his narrative centred on how his girlfriend liked his behaviour, that his control and the terror he inflicted gave her boundaries and meant she knew that he 
was the dominant partner which he believed made her feel more secure in their relationship.

I don't know, I got the feeling she liked it [violence], it gave her boundaries, gave her security. She knew I was in charge and, like our kids, you have to set boundaries and then if they break them you have to follow through with what you say you'll do. Kids respond to that, they push boundaries to check you're watching, they want to know you are paying attention. Jane was the same, if she broke a rule then I had to follow through. There's some security in that, I'm watching and I'll follow through. A dependable guy. (Ricky, IT)

Another way perpetrators minimised responsibility for their violence was to blame it on a lack of self-control. A common language employed by male participants who used violence against their dating partner was around their loss of control. It was part of a narrative that used phrases such as "I just lost it" or "I just snapped". For situational couple violence males and female participants, the explanation for aggression and violence frequently centred around expressive explanations that were emotionally driven such as a loss of control. This was often in a particular situation or argument rather than a general pattern in the relationship. The need for control in situational couple violence was also often described as a combination of being angry and not being able to manage impulse.

I just lost it, we were having an argument and he just didn't get it and I was getting so angry and I just lost it and started hitting him in the chest and swearing at him. I just couldn't get through to him so I just snapped. (Beth, $S C V)$

For intimate terrorism, the inability for self-control was articulated well by Ricky. In the section above Ricky described his beliefs that his girlfriend liked the violence; in a sense he believed that his violence provided security and boundaries for his girlfriend. Here he implied that she had control over his violence, if she did not break his rules. Ricky justified his intimate terrorism further through his inability to control himself when he got into a rage.

If I do get angry it's not good for myself or people around me, I lose mental capacity, there's not much going on upstairs, I black out. I can't help what I 
do when that happens, I don't have control. I think it's something to do with a continuation of my teenage anger from hormonal imbalance. I really couldn't help it when I lashed out at her. She just needed to see the signs and leave before I got that bad. She needed to take some responsibility for hanging around when she could see I was getting into a rage. (Ricky, IT)

Within two SCV relationships, where the females were violent, there was a strong gender minimisation discourse, in other words female violence was minimised because "he just wouldn't be scared of her" even though she was trying to hurt him. This was a less common discourse but supported by societal norms that often minimise female violence as humorous and inconsequential. There is an acceptance of females engaging in this type of behaviour, which would be unacceptable if a male did the same thing.

Gender norms are quick to sanction or discount physically violent women, whereas physical violence is often a normalised practice of masculinity; men's physical violence is generally bestowed with power and considered threatening whereas women's physical violence is not. (Nybergh et al., 2016, p. 199)

Some female participants knowingly and deliberately used this belief that their violence would be discounted. Mary described her violence and how she used this gendered discourse to minimise its seriousness.

There was this time when I was drunk and I was yelling at him, "you're a fuckin' cunt" then I started hitting him in the face. But that was funny, like, that was the violence in the relationship. The alcohol brought it out. I don't remember it so I don't know what happened. He was the same size as me so it was quite good, it was fair game. I'm a chick so it's just not that bad, I can't do that much harm, right? No one will think it's an issue. (Mary, SCV)

Another female participant, who reported that she was the main instigator of violence in her relationship, also situated her narrative in a violence minimisation discourse to describe how she felt it was more acceptable for a female to be violent compared to a male. 
My violence is okay. If a girl is out all night and doesn't come home that night and he starts hitting her and I'm like what? But if he's out all night and she starts hitting him I'd still be, "Like what?", but if she's really at her wits end and she's like, "Hey can you just do the dishes" and he says, "No that's a girl's job" and she slaps him... I don't believe in women beating their partners but I do understand slapping. But it depends on the situation. We're all adults here, there probably shouldn't be any hitting at all. (Mary, SCV)

This also meant that these female participants were often not held accountable for their violent behaviour, as it was not taken seriously. In this sense, the female's violence was legitimised and minimised because she was a female, and was supported by common beliefs that it is men who are primarily the violent aggressors in relationships. This reinforces traditional female traits of being meek and unable to gain control over males (Nowinski \& Bowen, 2012).

However, this also meant that some of the male participants, who experienced violence by their girlfriends or where there was mutual violence in the relationship, felt degraded and made to feel like their experiences were not legitimate. Drake described how his girlfriend was violent within the context of a range of other psychological tactics. She would use personal information about him that she knew left him feeling vulnerable and this would impact on his self-esteem and self-worth. This left him feeling depressed and isolated as he could not admit to her violence, nor could he reconcile his reactions to the violence and to his inability to stop her.

I was quite fat, she called me names, and she hit me a lot, she laughed and said there was so much fat there I wouldn't feel anything anyway. She said I was a weak man because I didn't fight back. It was all true I guess, I was fat and wasn't man enough for her. And I couldn't tell anyone, I mean any guy who gets hit by his girlfriend and who is feeling sad and depressed because she calls him names, well that's pretty pathetic. I was pathetic, I didn't need anyone else knowing that. She was just real mean, I didn't have it in me to fight back, to stop her. I just took it like a pussy-boy. Pathetic right? (Drake, $S C V)$ 
Another narrative from young male victims was that of minimising the female's physical ability to cause harm and that no one would believe a female could be capable of harming a male. Javaid (2018) describes how societal beliefs hold that men cannot be victims without diminishing their successful attainment of masculinity, and how their victimisation challenges and contradicts the expected performance of traditional gender roles. Jack explained his experience of violence, and through the minimisation narrative.

Sometimes she'd get real angry and hit out. It's hard to explain, as a guy I could've probably stopped her if I got physical too, but that wasn't really me. But it did make me question if I was much of a man, letting a woman hurt me like that. And what would happen? If I tried to stop her I would be the violent one, no one would believe that a girl would start it, that a guy was just defending himself. There was part of me that probably felt stupid admitting that I'd been beaten by a girl. As I say that, it sounds ridiculous but I think there was something about not wanting to look weak, god it's hard to explain, I wasn't really scared of her hurting me physically, maybe hurting my reputation? (Jack, SCV)

Another perspective on this was given by Grant who described his same-sex relationship in which his partner was violent towards him. Through his narrative he relayed his views more broadly about same-sex relationship violence and the legitimacy of men as victims.

Grant - Males abuse to males? I think it plays out the same, there's always one person violent towards the other no matter who, if it's going to happen it will be one person even if they are the same sex.

Interviewer - What about a guy as a victim?

Grant - I don't think people think about it 'cause they don't see it, there isn't any coverage of same sex violence I don't know if it's 'cause people aren't aware of it or they don't care. You know out of sight out of mind kind of attitude

Interviewer - What about when a male is the victim? 
Grant - They say it but you don't take it the same way, as if it was a guy hitting a girl, even when a girl is abusive to a guy people just don't buy itthat it can't be harmful. It goes down to gender stereotypes. Guys are supposed to be all macho and strong yet everyone can be a victim, it will affect you either way. (Grant, SCV)

Here Grant described this gender minimisation discourse, where the disbelief that a man can be a victim overrides and de-legitimises their experience. This narrative was supported in analysis by Gillum and DiFulvio (2012), who surveyed 109 sexual minority young adults aged 18-24 years from a university in the USA. They concluded that in sexual minority relationships there is still a strong need to navigate prescribed gendered roles which construct the role of men as dominant. Young men's victimisation does not align with these gendered norms, which can lead to their experience as a victim of dating violence not being taken seriously. This suggests that examining same sex dating violence through a heterosexual lens may have limitations in fully understanding the unique elements of these experiences and that existing prevention programmes using heterosexual relationship concepts may be less effective for this group.

This section provided a detailed analysis of attitudes and beliefs described by participants that were supportive of and/or used to justify and minimise violence, by both victims and perpetrators. The analysis in this section was written in a more gendered way due to the different roles of men and women in the violence and how it was justified and minimised.

Violence minimisation was commonly used when female participants explained the violence they experienced, using phrases such as "he was an awesome guy", that he "really cared about me" or with tolerating language such as "it wasn't that bad". Play fighting and arguing was also used by some participants to minimise some violent and aggressive behaviours. This gender minimisation narrative was also apparent when talking about women's violence but from a different perspective, where their violence was described as acceptable because they were women and their violence was therefore inconsequential. Male perpetrators in this study tended to use narratives that implied or more directly blamed their girlfriends for their violence. 


\section{SECTION TWO: A STORY OF CONTEXT}

As discussed in Chapter Two, a key component in the literature is the importance of understanding the context surrounding acts of violence. Researchers such as Hamberger (2005) and Holtzworth-Munroe (2005) have posited that when context and patterns surrounding relationships and violence are examined together, a more complex picture is uncovered that can more fully demonstrate different patterns and experiences of violence. Within Johnson's (2008) typology this would imply that the experiences of those in situational couple violence would be qualitatively different from intimate terrorist relationships. Situational couple violence is believed to be more expressive - emotionally driven and based around conflict and mutuality of violence while intimate terrorism is instrumental - violence embedded in the need to assert or restore power and control with coercive controlling patterns that are experienced in a more gendered way with far greater negative impacts on the victims (Flood, 2019).

This section covers a number of contextual factors that were identified thematically throughout the interviews and were supported through the literature. They include narratives on who initiated the violence, the motivations for the violence and the outcomes and impacts from the violence. Through this analysis I looked to see if there is a strong argument for symmetry in violence within and across Johnson's typology categories or if there is a starker contrast in experience when this context is accounted for.

\section{INITIATING VIOLENCE}

One area discussed in the IPV literature is around the initiation of violence. Research around this is mixed but the argument put forward by feminist-based research around women's use of violence is that it is most often a response to their male partners' violence. In that sense, men are more likely to initiate violence and if a woman does use violence it is in response to their partner's violence (Swan \& Snow, 2006). This pattern was not found in this study for those in situational couple violence relationships, where a common theme was that when arguments or conflict occurred that sometimes erupted into violence, these could be initiated by either the young male or female. This was particularly evident for those couples that were living together. 
He called me horrible names, it would start on something small, then get big, he'd leave, then eventually come back and apologise. It was petty stuff that didn't really hurt. I guess the only way for him to get the frustration out was to be honest and brutal about what he thought of me. (Kathryn, SCV)

There was pushing and slaps in the relationship, I didn't hit back, but I did push back. Once he pushed me up against a wall so I shoved him back really hard. (Beth, SCV)

This differed from intimate terrorism relationships where the initial dating period was described by participants as a positive time when males were described as behaving at their best, where the controlling behaviours were seen as chivalrous and caring. These behaviours included their partner sending texts and using social media as a means of checking in on their partner. Other examples included driving and picking the victim up and restricting the victim's social interactions. The female intimate terrorism victims in this study described viewing these control mechanisms as chivalry and interpreted these actions as a demonstration of love at the start of the relationship. Laura described how the relationship started with her boyfriend acting like the "perfect boyfriend". However, as his behaviour became more intense and she started to challenge him, to gain back some independence and to not conform to his rules or to not behave as he instructed, he soon became more dominating and this was when physical violence began.

Interviewer - Was it something that progressed over time?

Laura - He was okay for a few weeks, I guess looking back I can see that the control started from the beginning. I just thought then that it was him being romantic.

Interviewer - Can you give me an example of something.

Laura - Um well, he said he wanted me to come over to his place, then he asked me to stay. I said I wanted to go home later and he wouldn't take meI'd had a couple of drinks, and he took my phone off me and told me he would take care of me. I thought at the time it was nice that he cared but I still had this niggle, I think because I had been drinking I didn't realise how bad it was. 
If anything had happened I wouldn't have been able to get away. He just manipulated every situation. Before I knew it I felt so trapped and I realised that I had been totally removed from things that were normal for me.

Interviewer - Can you explain that a bit more, removed you from things that weren't normal...?

Laura - Yeah he said my friends didn't like me and he'd heard them bad mouthing me, we never went out we just stayed at his house, sometimes he wouldn't even let me talk to my mum on the phone. Later I found out that he'd made it all up. That my friends had never said anything bad about me and he'd told them that I didn't need them anymore and that's why I wasn't getting back to them. They believed him, and I believed him, he was just a nice guy. That's what people thought of him. He could just turn it on and off when he wanted. It made me doubt how I felt. I was trapped. (Laura, IT)

Likewise Sammy described how amazing the start of their relationship was but how quickly her partner's kind and protective behaviours became controlling and 'suffocating'. Sammy was a 21-year-old student who had been with Tim for two years. They meet out clubbing and quickly formed an intense relationship. Within weeks she had moved in with him. She described the early days of the relationship as being fun, he was attentive, they did lots together, and she barely had time to hang with her friends. He encouraged her to miss classes at university so they could hang out at home together. They had lots of date nights in, but when she started suggesting they go out to parties, hang with friends, he pushed back. She realised that he was quite controlling and by this stage she was quite trapped in the relationship. Her belongings were in his flat and he knew all about who her friends were, her university and work schedule, passwords for some of her social media sites. He started to follow her, turn up to her work, and be waiting outside lectures. She became scared and when she told him she was moving out that's when she described that he first "lost it" and physically assaulted her. She had a strong network of friends who were concerned for her and acted as a protective mechanism, helping her to leave safely. He began to stalk her more and she often felt like he was watching her. He would leave presents at her new flat, which meant he still knew where she was. All this happened within the 
space of about four months so escalated quickly in terms of intensity, entrapment, fear and violence.

Ifelt like I was suffocating, that he was everywhere, I was always on edge. I knew I had to leave and get my life back but, I look back, I was terrified of what he might do, where he'd be, that he'd find me. I knew he could, at the start when he was so sweet and caring he did that by following me, knowing my habits and routines. This became his tool for stalking me. (Sammy, IT)

This section has demonstrated the differences participants described in how violence was initiated in the relationship between situational couple violence and intimate terrorist couples. Situational couple violence was more likely to occur during an argument that erupted into violence, and could be initiated by either the young male or female. Conversely initiation of violence in intimate terrorist relationships in this study was gendered and had a more gradual appearance, where the initial dating period was described by female participants as positive and the controlling behaviours were initially seen as chivalrous and caring, but became more negative and violent as the relationship progressed.

\section{Motivations FOR VIOLENCE}

Another contextual element is the motivation for the use of violence. A growing body of research centres on the debate of whether men's and women's use of violence is similarly motivated. As stated in Chapter Two, motives that have been identified in the literature include intimidation, control, self-defence, to 'show who's the boss', poor impulse control, poor conflict management and expression of feelings (Hamberger, 2005; Dutton \& Goodman, 2005; Pence \& Paymar, 1993). Within the range of motives there appears to be some overlap in the reasons given by male and females who perpetrate violence, while there also appear to be some commonly reported differences (Holtzworth-Munroe, 2005).

Feminist-based studies tend to focus on men using violence in an attempt to exert power and control while women's violence is explained as an attempt to defend themselves during violence or as a form of retaliation for violence, citing self-defence as the overarching motive (Hamberger, 2005; Pence \& Paymar, 1993). However, as more research on the motives for using violence has emerged, there has been a 
growing number of studies, particularly in the dating context, where women reported using violence to dominate and control their male partners, and where a small number of men reported their use of violence in self-defence (Hines \& Douglas, 2010).

Throughout the interviews young adults described a number of reasons for violent behaviour. These included beliefs about jealousy, traditional gender role conformity and expectations, and ownership and maintaining power within the context of masculine performance. This is where there were more distinct differences in this study between intimate terrorism and situational couple violence couples, whereby situational couple violence often occurred around the need to control a situation, or due to poor conflict management, while intimate terrorism violence was often motivated by a much stronger need to control the individual within the relationship at all times.

One of the most common motives for violence and control in this study was jealousy. Both male and female participants described the manifestation of their partner's jealousy as a motive for violence, whether overtly displayed or felt in an underlying sense by participants.

$$
\begin{aligned}
& \text { Sue - Jealousy to a degree is good, like not over the top } \\
& \text { Interviewer - So what's good and what's over the top? } \\
& \text { Sue - Over the top is texting "where are you", "who are you with", "when are } \\
& \text { you coming home" } \\
& \text { Interviewer - So what you just described, what you have... } \\
& \text { Sue - um yip - [laugh] yeah I guess that is what David does [laugh]. }
\end{aligned}
$$

There appeared to be some level of normalcy to the presence of jealousy in dating relationships as described by participants, however within controlling and violent relationships there were negative implications from the enactment of jealousy. Capaldi, Kim and Shortt (2007) reported that for younger couples where there may be lower levels of commitment and less relationship stability, jealousy is more likely to be present. In a study by Adams and Williams (2014) they reported findings from focus groups with 15-17 year old Mexican American youth: 
Adolescents described jealousy as a phenomenon that permeated their partnering experiences and as pivotal in how they defined and experienced violence in their dating relationships... Rather, jealousy surfaced as the most prominent relationship problem discussed among adolescents across the sample and in this sense, all were prone to it. (p. 304)

This was supported by Rebecca, who described how her boyfriend's jealousy led to arguments and aggression.

For us, jealousy was a big problem, I was just not trusted. I couldn't be friends with another guy. If he thought I was with someone I wasn't allowed to be with he would turn into a scary monster. He knew he was a jealous guy, I would see him get angry and aggressive about the situation. He'd get this look in his eye, the way he talked got quite harsh, short, like really blunt. Then there would be this massive explosion and that's when I didn't know what was coming next, would he just storm off, say something mean or maybe this time he would hit me. (Rebecca, SCV)

Jessica described how her boyfriend was jealous of her hanging out with certain groups of friends and how she had to change her behaviour and avoid certain social situations to ensure his aggression would not boil over into physical violence.

I changed my behaviour because of his jealousy. I would only hang out with certain people. He'd have a bit of a rage. Then I'd apologise. I had to apologise to keep the peace. I guess in the end it was just easier to hang out, mostly at his place and watch TV instead of going out with my mates. He's very jealous and protective. (Jessica, IT)

Jealous reactions were not limited to males, with Jack describing his girlfriend's show of jealousy in their relationship and the behaviours that followed.

I hugged a female friend and my girlfriend went absolutely off at me, absolutely nuts. There were lots of similar incidents. Especially with Facebook posts, there'd be a picture of me and she would think she knew what had happened, nothing had happened. There was no trust, she expected me to be unfaithful. If I'd been out and she didn't know who I was with she'd turn up to 
my apartment and scream at me, she would want to come in and see if there was some other girl in my bed. (Jack, SCV)

This response to jealousy appeared to differ between situational couple violence and intimate terrorism couples, although jealousy created a significant impact on the relationship and subsequent behaviours for both groups in this study. However, an underlying element of jealousy in intimate terrorism was based on strong beliefs and gendered attitudes motivated by the need to perform successful masculinity. Central to Johnson's (2008) theory of men's intimate terrorism is the enactment of masculinity displayed through beliefs of ownership and male superiority. These beliefs hold that men should be superior and dominate 'weaker' groups, particularly women (Brown et al., 2010). This dominance, as seen in many violent relationships, is enacted through psychological harm and violence. It was not surprising that such views of ownership and control were evident in respondents narratives about male intimate terrorism respondents in this study.

I don't get jealous but he does - he gets protective then gets jealous. He gets pretty angry if I don't obey, like real angry. He doesn't allow me to drink because he's not there to protect me if I get silly. It's okay though, it's his job to tell me how to behave and make sure I'm not disrespecting him. (Sammy, IT)

Yeah I didn't like her talking to other guys. I don't know, maybe I was jealous or something, I just needed to know what she was doing and who she was to check she was breaking the rules or not... rules, well it's pretty clear about how a girlfriend should act, and what they should do. So I'd keep an eye on her. That's the job of a boyfriend; to check in on them and make sure they're doing the right things and not embarrassing themselves. (Regan, IT)

Laura described how she felt as a result of her boyfriend's toxic masculinity and the language that he used to demonstrate his power over her.

I guess I was made to feel, I mean I wasn't his girlfriend but his missus, like Outrageous Fortune. He would totally say to me "I own you" that I was his. I was just another possession really. (Laura, IT) 
The tension between modern views of gender equality and contradictory beliefs about maintaining traditional gender-based norms and attitudes in relationships was also more broadly described by participants. Results in Chapter Seven presented a common narrative where participants described the desire for an egalitarian relationship, but female participants' narratives quickly shifted to describing situations where they took on socially constructed gender roles and ended up in roles "like their boyfriend's mothers", indicating that they often took on more traditional gender-based ideologies (Lamont, 2014).

Within the analysis of violence, this conflict around gender role beliefs and ideologies led to a number of arguments that resulted in physical violence or threats within some situational couple violence relationships. Female participants almost uniformly expressed feeling that there was an expectation that they would adopt traditional female roles within the relationship. Female participants described that when they continually challenged these traditional gender roles, such as cooking meals and cleaning, this often led to arguments, and in some circumstances, physical altercations.
Sometimes I thought "I'm just like my mother", all I do is cook, clean, all those jobs. I felt like his mum, not his girlfriend. I guess we just developed into those roles, and then when I tried to change this it led to arguments. I guess I should've said no from the start and not set it up as normal. It just seemed to happen so naturally. (Beth, SCV)

For intimate terrorism couples, increased violence and other tactics also emerged when the female started to challenge these traditional gender-based norms and roles. In the early stages of their relationships two intimate terrorist participants described their boyfriends' chivalrous gestures such as buying them flowers and how this showed them he was a respectful and caring boyfriend. However, over time these female participants realised that these behaviours were ways to draw them in. The enactment of traditional gender-based scripts by the men displaying such gestures meant they had, in fact, taken on traditional roles in the relationships, and this was starting to impact on their freedom and individuality. 
As Sammy described, by the time she realised she had "become his mother" and had very little power to make decisions for herself, her boyfriend had also managed to isolate her and he had significantly eroded her self-esteem through constant put downs about her appearance and level of intellect. This left her feeling even more trapped in her 'new' identity within the relationship and there was no resolution but for her to conform to his expectations.

I ended up just being like the good housewife, like something from a 1960s show. I was expected to keep house, like I was in a time warp. It started out ok, like I wanted to be a good girlfriend and help out but then there was some unwritten expectation that it was my job and not his. I felt so bad about myself, he made me doubt myself and so I just did something he said I was good at. I had to anyway, at that point I was so trapped that if I didn't take on the good girlfriend role then he would get so angry and that would lead to him just getting even more nasty. It was such a trap, I was so trapped. (Sammy, IT)

While there was a strong narrative of conflict and violence motivated by jealousy and intimate terrorist men's use of violence to sustain power and control by forcing women to conform to gendered-roles, there were a number of female participants who described their deliberate motivation to cause violence and 'chaos' in their own right, and not in response to their partner's violence. In other words, some young women in this study reported their use of violence as deliberate and not as an act of self-defence or a more passive sense of fighting back. This was consistent with studies such as Harned (2001) who reported that both male and female participants described their use of violence as a way to gain control over the other person, to feel more power and to get their partner's attention.

Female situational couple violence participants described a number of ways in which they deliberately enacted control over their partner. Mary described her intent as being calculated and manipulative, she knew how to get her own way. Mary also articulated clearly that she knew she could get away with this behaviour because, as a female, no one would believe that she had that much power over a male.

I think people believe that its sort of acceptable for women to hit men, it's only a punch in the face when it's the girl but if it's the other way around it's the 
end of the world. So I knew that hitting him wasn't a biggy because people would be ok with it, I mean I'm a harmless chick right. And it got such a reaction from him, I loved the drama and the chaos I could cause. He didn't know how to handle me or what to do to respond, I knew he didn't have a violent bone in his body. I felt so in control, so powerful 'cause he was really a big teddy bear and I could show him who's the boss. (Mary, SCV)

This need for control and how young women can enact it was supported in the narrative from Mary who was classified as situational couple violence. Mary reported her use of control in their dating relationship with her motives of gaining power and control. While this control was not rooted in patriarchy and male privilege, it was still strongly embedded in a more general need to be in control of her boyfriend.

Like I said, I was definitely not always the best girlfriend, maybe a bit violent. It just felt good to be able to control David. He was meek and timid; he would never do anything to hurt me. I knew what his weaknesses were and I used those to manipulate him, to get him to do what I wanted. Sometimes when I flipped out I hit out but that was to show him that I was the boss in the relationship. I had so many of my own hang-ups that it was good to be able to be in control of someone else and have some power in one area of my life. He was an easy target. (Mary, SCV)

The need for control and the more continual pattern of manipulation and violence that Mary described throughout her interview was not common in the other situational couple violence relationships in this study, although three female participants did describe patterns of violence and control that became regular aspects of their relationship.

It just became more normal, it happened more often and became the way we would argue, almost like a movie script, in that scene where you would argue and then there would be the pushing and shoving sequence, it would get aggressive, then we would say sorry and wait for the next scene where that would play out again. (Emma, SCV) 
It was more likely that it would end up physical, the physical stuff became frequent, just the new normal I guess. That became the way he would get his emotions out, it worked so he kept doing it. (Sue, SCV)

We'd argue and I guess it just became more normal. He'd get jealous or be telling me what to wear and I'd push back and then it would get a little bit, like, physical. Not major or anything but it happened a few times, it became part of the argument. Part of how we'd resolve it. (Rebecca, SCV)

This section has presented the types of violence experienced by these young adults and the motives behind them, suggesting that there are some differences in the impact of violence between situational couple violence and intimate terrorist relationships. The next section extends this analysis to consider how the narratives surrounding the impact of conflict, violence, and aggression in dating relationships differed between situational couple violence and intimate terrorism participants.

\section{THE IMPACT OF VIOLENCE}

Central to discussion on gender symmetry and context is the impact of the violence and differences experienced by males and females in terms of severe physical injury, medical treatment, psychological outcomes and level of fear (Holtzworth-Munroe, 2005; Harned, 2001). Dating violence has been shown to produce fear, injury and emotional health consequences for both males and females, however these studies often show the negative impacts to be far greater for females (Borsky et al., 2016).

Most participants described experiencing one or a range of dating violence acts and a range of motives for the use of violence - there was also a range of impact described by participants. While not wishing to minimise the seriousness of any of these experiences, the impact was often less severe both psychologically and physically for those categorised as situational couple violence. Notably, however, a large number of participants described lasting mental health issues resulting from negative relationship experiences.

One of the most visible impacts of violence is the experience of injury. Research suggests that injury is more likely to be inflicted by men resulting in women experiencing greater severity of injury and needing to seek medical support (Hamberger, 2005). Such evidence supports the view that women bear a far more 
significant brunt of the physical impacts of violence, whether they are a victim-only or involved in bi-directional violence.

There were qualitative differences in the nature of injuries between situational couple violence and intimate terrorism relationships. Seven of the ten situational couple violence couples reported experiencing injury. For most of these young adults the injury was minor, which is consistent with Johnson's (2008) theory that situational couple violence relationships largely experience less severe violence. However one participant described severe injury in the context of a situational couple violence relationship. Sarah recounted an incident where her boyfriend became violent and she ended up having to go to the Accident and Emergency Department as a result of an argument.

We had a few different times where our arguing got too much. One night we were going at each other, I can't even remember what it all started about, but I pushed him and he grabbed my arm. I don't quite remember the sequence of it all but he pushed me back hard and I hit my head on the corner of the desk. The blood was crazy, I was bleeding quite a bit. We decided to go to the after hours to get it sorted, make sure if wasn't real bad. Simon didn't come, I didn't want him to get in trouble so I rang my friend and she drove me. I just needed a couple of stitches. It brought me a few days grace, he was super nice after that. (Sarah, SCV)

For intimate terrorism participants, minor injury was a common occurrence but this was often coupled with the experience of severe injury. In four of the six intimate terrorist relationships participants described severe physical injury resulting from physical violence, with one of the female partners needing to visit a medical professional and one incident where the police were involved following a violent attack.

In the quote above, Sarah described the most severe incident of physical violence where her boyfriend severely assaulted her resulting in her admission to hospital. It was as a result of this attack that her mother became aware of the violent relationship and they were able to work with the local police to charge her boyfriend with assault and for her to get support to safely leave the relationship. 
Below, Ricky describes more severe physical injury within the context of a wider argument around access to his children. He became increasingly violent, and then turned his aggression on himself. The result for his girlfriend was significant facial injury, and for him, as the result of self-harm, admission to ICU and potential for brain injury, followed by the police charging him with assault.

I hit her, she was screaming, I was yelling. It was chaos. She started yelling "I'm just going to kill myself". She grabbed a belt and put it around her neck so I grabbed it and put it around my neck and said "that's how you do it". I passed out, I broke a bone in my neck, I was having seizures. I went into a state of euphoria. The cops came and they had to wrestle me down and put relaxants in me. I don't remember much about that part, apparently during all that I broke the back door and her nose. Maybe she did that to herself, I can't even remember. I just woke up in intensive care. Apparently I wasn't far off being brain dead. The cops did nothing about her assaulting me, they just turned a blind eye to it. But not to me, I got charged for it all. I've been really bitter about that, really angry. (Ricky, IT)

While 13 of the 16 respondents in this study experienced injury, the main difference between narratives of situational couple violence and intimate terrorism was the use of language to describe the impact of the violence. While situational couple violence participants described being scared within particular situations, it was the distinct mention of the word "terror" by intimate terrorism victims that captured the additional level of impact the violence had within a pattern of control.

The experience of terror is a defining feature of relationships that have high levels of coercive controlling behaviours and are designed to intimidate and frighten their intimate partner. Instilling terror allowed perpetrators to assert their power and strength and forced victims to conform to behaviours expected by their romantic partner and to modify their behaviours to avoid violence and injury (Hamberger, 2005). In this study, participants' narratives of fear and terror appeared to be a gendered experience, particularly in the context of intimate terrorism where male perpetrators used a range of tactics including more severe physical violence. These findings were consistent with Dutton and Goodman (2005) who also found that the 
experience of terror was more commonly described in relationships involving high levels of coercive control.

The young women participants who were with an intimate terrorist partner described a much stronger pattern of fear, unpredictability and terror compared to young women with situational couple violence partners. The behaviours of the perpetrator were often described by the female participants as deliberate and calculated and left them feeling powerless to talk to anyone or to leave. The intimate terrorism victims in this study felt these controlling tactics were more strongly motivated by the need to control every aspect of their lives in contrast to situational couple violence participants whose experience of aggression and violence was more easily explained within situations of conflict and poor conflict management skills.

We met through some mutual friends. It's always clearer when you look back. When I met him I thought he was amazing, he was so into me. Caring and paid me loads of attention. We started doing everything together. But then it started to get more intense, where I couldn't do anything without him and he didn't trust me. Then the anger started to show through, when I'd done something he didn't like, he didn't agree with, he just couldn't hold it in. When he started pushing me around it was scary, but more because I didn't know what would set him off. He was really big on being respectful and following his rules. I just tried to do that, but even then sometimes something small would set him off. (Sammy, IT)

This added terror and unpredictability appeared to make it much more dangerous for intimate terrorism victims to leave the relationship, as there was a greater perceived risk of harm by these participants. This is supported by research that highlights the actual increase in harm experienced by young women when they leave abusive relationships. Statistics across jurisdictions also show that leaving a violent relationship can increase the risk of further harm and even homicide (Bruton \& Tyson, 2018). For Laura, her intimate terrorism boyfriend's beliefs of ownership and control led her to feel she was unable to safely leave the relationship. Laura said that when she was in the relationship she felt like she had no way out, that she could not leave unless he chose for her to, she felt trapped, isolated and vulnerable as a result of the control she felt her boyfriend had over her. 
I couldn't leave, I couldn't get away, I was there to make him look good. To service his needs. I was the item that had to be there. There was no leaving, no getting away. (Laura, IT)

For participants in situational couple violence relationships, there was some level of fear reported within the context of a fight but this fear often ended outside of those incidents. The violence was largely situational-based and was often more predictable. The violence was often minimised by participants as they felt that, while there may have been some level of control and aggression, it did not have a lasting impact on their wellbeing. So while participants described feeling scared or fearful at the time the violence occurred, or felt caged in by continued monitoring and surveillance from their partner, they generally described feeling that they had far more choice in whether they would stay in the relationship and most did not describe ongoing fear of their violent partner when they did decide to end the relationship.

Look, I guess we had loads of ups and downs. Looking back he did check up on me a lot, and there were a few times we got into some argy-bargy. I mean it was a little scary in the moment, when were really fighting, but it didn't last long. We always made up and most of the time he was cool, we were ok together. When I decided I wanted out he was pretty upset but I didn't feel like he was gonna do anything cray cray [crazy]. It just wasn't working out and I was better off on my own. So we broke up. We moved on. (Kathryn, SCV)

While the impact of violence was largely described as being significantly greater for females, both female and male participants within some situational couple violence relationships described female violence and the psychological impact this had on men as they tried to reconcile the disjoint between being male and being victimised.

Well, like when I get angry and I've reached my limit, it's like I just don't know what to do so hitting is the next option. And then there was a knife there so I thought I could use that to freak him out more, if I held it against my wrist. It worked, he got totally freaked, I mean I only did it once or twice and not to hurt him but to freak him out. I guess I wanted to frighten him and it did have that effect. (Mary, SCV) 
While this was not at the same level of terror that was described by intimate terrorism, it does highlight the need to acknowledge and support young men who experience coercive and violent tactics by female partners. Espinoza and Warner (2016) described how males who are victimised are placed in a role that moves them away from traditional gender expectations where they are supposed to be invulnerable. There is significant negative stigma attached to men who let their girlfriends abuse them. The view that men who are abused are weak or wusses is founded in gendered beliefs, which also lead to women's violence often being seen as less serious or ignored, providing further validation that male victims should feel shame for letting their girlfriends treat them like that.

This section provided detail around the context of the violence that these young people described. The way that violence was initiated into the relationships differed between situational couple violence and intimate terrorist young adults with situational couple violence describing violence as the result of the need to control a situation or due to loss of control of a situation. However, the initiation of violence in intimate terrorist relationships often began in the context of chivalrous and kind gestures that were the foundations of strong controlling and potentially violent relationships.

A narrative of jealousy was common across most relationships in this study, for both young women and men, as was the effect it had on these relationships. The impact of gendered roles and beliefs was also part of the motivation narrative where the desire to regain equality of roles led to arguments and, for some couples, violence.

The defining element where intimate terrorist and situational couple violence were not comparable between genders was in the experience of injury and terror. Overall injury was reported across most interviews, but this was mostly minor in nature and caused by both males and females in situational couple violence relationships. Severe injury and the use of the term terror was particular to female participants experiences in intimate terrorist relationships that were unpredictable and motivated by strong patriarchal beliefs aimed at control. This differed significantly from the impact narrative in situational couple violence relationships in this study. 


\section{CHAPTER SUMMARY}

This chapter has presented the narratives of young people demonstrating the role that attitudes, beliefs and context play in the acceptance, justification and impacts of dating violence and the differences found between those in situational couple violence and intimate terrorist relationships in the way this was described.

Overall it appeared that in some circumstances Johnson's typology holds, such as the differing motives for violence, and the injury and terror caused by violence between situational couple violence and intimate terrorist couples. However, while areas such as monitoring and surveillance, and justifications and excuses show differences across the typology categories in this study, the young adults' narratives also demonstrated the more general role of gender and the performance of masculinity and femininity which was a strong defining feature in the experiences of dating across most relationships rather than distinguishing features of the different typology categories.

The analysis in this chapter, and in Chapter Eight, provide a strong foundation for discussing the validity of Johnson's typology in the context of dating violence. While this conclusion is somewhat brief Chapter Ten provides an overall discussion of the applicability of Johnson's typology. It brings together strands from across this thesis and details the summary of findings from the literature, quantitative and qualitative chapters in more detail. 


\title{
CHAPTER TEN - SYNTHESIS OF FINDINGS
}

\author{
There is a need to understand the dynamics, nuances and components of \\ intimate partner violence with the idea that this critical comprehension is vital \\ in creating intervention and prevention models that befit the behaviour. \\ (Pence \& Dasgupta, 2006, p. 4)
}

This thesis is one of the few New Zealand-based studies that reports on young adults' experience of violence in the context of contemporary dating relationships. This chapter provides an overall synthesis and discussion of the findings, weaving together the strands of data including references to the literature review, survey analysis and interview material. I discuss supporting and contrasting evidence gathered through this research process and build a response to my research questions.

My main motivation for this thesis was to understand more about the role of gender within intimate partner relationships, particularly in the context of young adult dating relationships. To do this I explored the utility of Johnson's (2008) typology of violence that he developed as a way to reconcile the differences between studies that found gender symmetry in the perpetration of violence and those that did not.

This research identified two overarching findings. First, there is a high prevalence of young adults experiencing dating violence in the social context of New Zealand. Second, the analysis is largely supportive of using Johnson's typology to reconcile different experiences of dating violence and to understand different patterns of dating violence - although this comes with some considerations and cautions in its wider application.

\section{THE SOCIAL CONTEXT - A BACKDROP TO DATING AND VIOLENCE}

My focus on young adulthood was a deliberate attempt to understand dating and violence in the context of young people navigating their way from adolescence into adulthood. As detailed in Chapter Eight, the experience of dating violence was inherently linked to the contemporary social context as described by young adults. This social context was embedded in a dominant drinking and hooking-up culture that 
has shifted dramatically over the preceding decades (Hutton et al., 2013). Throughout the interviews, participants described less traditional socialising and courtship behaviours, within the backdrop of more liberal attitudes and social norms supporting drinking alcohol and casual hook-ups. Participants described the almost universal culture of engaging in 'hook-ups' with relaxed attitudes to casual sex. However, within this culture there was a strongly perceived requirement to maintain gendered scripts when initiating and progressing through a relationship. As such, there was a need for participants to manage expectations of engaging in romantic encounters and forming romantic relationships within cultural norms of what is appropriate, norms which appeared to be grounded in traditional gender roles and double standards.

However, the engagement of participants in the dominant drinking and hooking-up culture meant increased engagement in more harmful behaviours such as binge drinking and having sexual encounters with people they may have only just met. Analysis in Chapter Seven demonstrated that the majority of interview participants described going out, drinking excessively, hooking-up, and through this process potentially forming dating relationships. In fact, alcohol consumption was a common theme that ran across all the interviews in this study. Most of the young people described how they went into town or to parties and actively sought to get drunk. While research into drinking behaviours shows that excessive drinking is not unique to young adults (O'Keefe et al., 2014), there is a greater likelihood of negative outcomes for young adults from binge drinking compared to older age groups, as young adults described drinking often in more public settings, together with the casual sex and hooking-up culture (Schwartz, 2016).

Alongside these more relaxed and liberal attitudes towards drinking and hooking-up was a narrative around the significant impact technology, the internet, social media and popular culture had on shaping and reinforcing attitudes and beliefs around young people's self-identities and relationships (Hendrickson, 2007). As detailed in Chapter One, the last decade has seen an increased reach and influence of technology and media and its use as a way to stay in touch and keep connected. Social networking sites like Facebook and Instagram are part of everyday lives with nearly $70 \%$ of American adults estimated to have a social media profile (Seidman, Roberts, \& Zeigler-Hill, 2019). Since the interviews for this thesis were carried out in 2013, it is 
estimated that online dating has tripled for those aged 18 to 24 years, with over $27 \%$ using this form of technology to find a romantic partner (Hoffman, 2018).

Interviewed participants described the use of technology and the interaction with a range of social media as an ordinary part of everyday life.

Young adults in this study described the influence of technology as not just messaging and advertising focused on what women and men should look like, how they should behave and what they needed to be successful, but the influence of wider media as an educational tool to inform them about what relationships should look like and how to behave in a relationship. Participants described the often confusing and conflicting messages about what to expect in sexual and romantic relationships and struggled with what was a 'good' relationship, and what was not acceptable in a relationship. Participants are operating in an environment where the internet, social media and popular culture can conspire to misinform them, particularly when 'education' comes from sources such as "reality" television, popular culture reporting or pornography which glamorises and normalises excessive drinking, reinforces harmful attitudes and beliefs, and normalises violence and sexualised behaviours (Keene, 2019). These influences made it harder for participants to navigate their way to positive images of themselves, their peers and their relationships (Paynter \& Leaper, 2016).

This social context provided a backdrop to the experience of dating violence, which this study showed was a reality for a large number of participants. What this thesis has highlighted is that the contemporary social context that young adults in the study described navigating through was complex as more modern expectations were magnified through technology and media, yet still sit alongside more traditional expectations about dating roles and gender norms.

\section{THE HigH RATE OF DATING VIOLENCE IN NEW ZEALAND}

Within this social context this study found that a large majority of survey respondents experience some form of violence in a dating relationship, from more minor experiences to dating relationships characterised by severe and terrifying violence. Analysis of the survey data presented in Chapter Five found that over three-quarters of young adults respondents were a victim of at least one act of violence in the 12 months prior to the study and eight out of ten young adults reported perpetration of at 
least one episode of violence in the 12 months prior to the study. While most of this violence was more minor in nature, some young adult respondents experienced a range of more severe forms of violence. The total absence of violence was only reported in around $20 \%$ of dating relationships, demonstrating the pervasiveness of violence and the need for this issue to be recognised as one of significant concern. These survey findings also demonstrate the dyadic nature of dating violence with a large proportion of respondents describing their dual role as both a perpetrator and victim of violence, highlighting the complexity in how we describe and intervene in this type of experience.

Survey respondents reported that one type of violence was seldom experienced in isolation. The overlap between types of violence including psychological aggression, physical violence and sexual coercion showed that physical assault was nearly always accompanied by psychological aggression, as was sexual coercion. A number of survey participants had experienced all three types of violence in the past 12 months at the hands of their current or ex-partner. As reported by Cale et al. (2016), physical violence rarely occurs in isolation and studies such as O'Leary and Maiuro (2001) show that physical violence is often present with psychological violence. This pattern was supported by participant narratives.

Of note was the high rate of sexual coercion revealed in the survey, with one-third of female and a quarter of male participants reporting being victims of sexual coercion. As described in Chapter Eight, sexual coercion covers a range of non-physical behaviours used to force someone to perform sexual acts. The rates of sexual coercion reported in the online survey were supported by the qualitative interviews, which revealed a common experience of willing engagement in unwanted sexual activity. Within the contemporary social context of hooking-up, young women interview participants discussed how they consented to sexual activity even though they did not desire it. This was linked to beliefs that support the need for young women to conform to gendered expectations and to fulfil male sexual needs. The most common narrative around willing unwanted sexual activity was one where women did not want to disappoint their partner or to cause an argument. Katz et al. (2006) reported a similar description of young women's willing engagement in unwanted sex where 
they reported consenting to this activity to avoid feelings of tension in the relationship, to promote intimacy and to satisfy the needs of their boyfriends.

While willing unwanted sexual activity and examples of coerced sexual activity were relatively common in this study, forced sexual activity was less commonly described and when it was, it was in the context of young women with male boyfriends. It was undeniable that sexual coercion was more gendered than other forms of violence, and more likely to be in the context of intimate terrorism relationships. Throughout the interviews no young men in heterosexual relationships described being victims of sexual coercion. Within the intimate terrorist narratives, young men described their use of sexual coercion as embedded in male gendered beliefs of women meeting their sexual needs and feelings of male ownership.

In summary, this study demonstrated the common experience of violence that occurred in dating relationships for study participants. The intersection of the current social context of hooking-up and drinking to intoxication, and the proliferation of technology and media, created spaces for dating violence to occur in a number of ways for participants. These include the additional pressure to maintain an image that is encouraged by current social norms and acted out through social media, as well as technology emerging as a more recent tool that supports the surveillance and monitoring of dating partners.

\section{THE UTILITY OF JOHNSON'S TYPOLOGY}

The confirmed experience of dating violence by young adult in this study provided strong grounds to apply Johnson's typology in this current context. The descriptive survey results in Chapter Five showed mixed findings around the role of gender in the experience and impact of violence, with patterns of gender symmetry evident in some relationships while a more contradictory pattern was found in other relationships. Applying Johnson's typology was an attempt to see if a more nuanced understanding of the complexity of dating violence in this study could be developed.

The central element of Johnson's typology was to reconcile the differences between research that has demonstrated male perpetrated violence in the context of gender inequality and the use of male authority and domination through coercive control versus research that suggests that there are equivalent rates of victimisation and 
perpetration experienced by both men and women. As detailed in Chapter Six, Johnson's typology protocol was applied to my survey data, where participants were classified as either non-violent, situational couple violence or intimate terrorism. Partners were also classified into these three categories. The results from the survey found that 144 respondents reported being physically violent, with $9.0 \%$ classified as intimate terrorism and $91.0 \%$ classified as situational couple violence. Similarly, there were 122 dating partners who were reported to use physical violence; of these partners $24.6 \%$ were classified as intimate terrorism and $75.4 \%$ classified as situational couple violence.

\section{THE ROLE OF GENDER}

At first glance the survey findings indicated support for the notion that both women and men experience some forms of dating violence and at similar rates, as both perpetrators and victims. However, further analysis looking at the proportion of men and women who experienced different types of violence and the motivations and impact of that violence showed a more pronounced gendered picture. The current debate on female equivalence to male violence raises question of whether gender symmetry implicitly means that the motivations and impacts of violence are the same for women as men (Enander, 2011). This requires a focus on understanding context and allowing analysis that identifies different groups of experiences. In this study the gendered picture became more pronounced in Chapter Six once the typology was applied. Through the typological lens, gender became a significant factor when looking at the distribution of the participants classified as situational couple violence or intimate terrorism. As detailed in Chapter Six, female participants were significantly more likely than male participants to report levels of violence and control that met the classification of situational couple violence. In other words, females were more likely to be classified as perpetrators of situational couple violence compared to male participants. Conversely, female respondents were less likely than male participants to report levels of violence and control that met the classification of intimate terrorism.

This pattern was reinforced through the interviews. There were ten interviewees whose relationship was classified as situational couple violence - four where both partners in the relationship were classified as situational couple violence, three 
couples where the female participant was classified as non-violent and their male partner was classified as situational couple violence, one same-sex relationship where the male respondent was classified as non-violent while his male ex-partner was classified as situational couple violence. The final two cases were where the male was classified as non-violent and their female partner was classified as situational couple violence.

In contrast, the pattern for intimate terrorism was more likely to be uni-directional violence where men were classified as the violent partners in all five of the couples in this category, and the male was classified as intimate terrorism in the one mutual violence case while their female partner was classified as situational couple violence.

However, the survey analysis showed that some female respondents did commit physical violence in combination with high coercive controlling behaviours. While these were extremely small numbers ( $\mathrm{n}=5$ females), results do show that women can exhibit violence and coercive control that is consistent with this classification. While there are range of reasons are reasons posited to explain differences in the reporting of gender, women's use of violence in dating relationships cannot be discounted.

\section{CONTEXT MATTERS}

The growing controversy of women's use of violence more generally has led to significant debate on its equivalence to male violence and poses the question of whether gender symmetry implicitly means that the motivations and impacts of violence are the same for women as men (Enander, 2011). Research investigating the contrasting motives and outcomes of violence by men compared to women showed differences in the consequences of violence. Such studies suggest that while there may be mutual violence within relationships, this does not mean that the motives and effects of men and women's violence are equivalent (McHugh et al., 2005). The interview analysis provided the platform to test whether there were qualitative differences between people in the situational couple violence and intimate terrorism groups.

\section{INITIATING VIOLENCE}

One area that shed more light on the gender symmetry debate arose through an analysis of patterns of violence and whether there is equal likelihood of men and 
women initiating violence in the intimate relationship (Hamberger, 2005). As detailed in Chapter Nine, in agreement with Johnson's findings, the current study did not find a strong gendered pattern in the initiation of violence within situational couple violence context, with both female and male respondents reporting their role in initiating violence (Kelly \& Johnson, 2008).

In contrast, the initiation of violence described by participants in intimate terrorism relationships had a different pattern, with young women describing how it later became clear that young male intimate terrorism perpetrators' chivalrous and kind gestures at the start of the relationship were in fact early signs of coercively controlling behaviours. It became apparent that these behaviours were actually the beginnings of a more regular pattern of control that could escalate to physical violence (or threats of) and be used to reinforce relationship power if other controlling non-violent acts did not get the partner to conform.

\section{MOTIVATIONS FOR VIOLENCE}

Another theme in the current study that shed light on the gender symmetry debate was to focus on the motives for committing violence towards an intimate partner. As reported in a number of previous studies that have investigated motivations for the use of violence, there are diverse motives for both male-perpetrated and femaleperpetrated violence (Straus, 2008). Those that have been identified in the literature include intimidation, control, self-defence, to 'show who's the boss', poor impulse control, poor conflict management and expression of feelings (Hamberger, 2005; Dutton \& Goodman, 2005; Pence \& Paymar, 1993). In this study intimate terrorism couples reported violence that was more strongly motivated by the need to uphold and enforce gender roles, and the general belief of men's right to control their girlfriends. Male partners classified as intimate terrorist had a strong expectation that their female partners would fulfil traditional roles and that young women would conform to these norms to avoid negative consequences.

Conversely, and supportive of Johnson's theory, situational couple violence was much more likely to be motivated by the need to control a situation or through incidents of conflict for young adults in this study. It was also motivated through young adult participants' interaction in social contexts that made the likelihood of 
violence higher, particularly when alcohol and casual sex were present. In this sense, young adult participants in situational couple violence relationships described how violence could occur at different points in the relationship as either a one-off incident or strongly situated in a contextual scenario of conflict that got 'out of control'. Building on this, conflict in situational couple violence relationships was commonly centred around the desire for gender equality in the relationship. Situational couple violence couples that struggled with relationship equality found this led to conflict and, for some couples, violence. This narrative was distinct from intimate terrorism where equality was less likely contested or if it was, it was responded to through the intimate terrorist's use of coercive or violent behaviour to stop the young women from pursuing this goal.

An additional theme in the current study that shed light on the gender symmetry debate was the range of excuses and minimisation tactics used to explain the experience of dating violence and how they differed within a gendered analysis. Within the different excuse and minimisation narratives that were reported in this study, male intimate terrorism perpetrators were more likely to minimise their behaviour and blame it on their female partners by drawing on strong gender-norms and violence-approving attitudes. The types of excuses and minimising language stemmed from the external factors and the young men using such phrases as "I lost it", or "she liked it". Young men's perceived lack of self-control was also used as an excuse by perpetrators for their violent behaviour, more strongly for intimate terrorist males whose narrative was around how they "just snapped" or "lost mental capacity". The two male interviewees who were classified as intimate terrorists also justified their violence by directly blaming their girlfriends, explaining that their violence was necessary to enforce "boundaries" and to teach their girlfriend to be a "good girlfriend". In other words, male intimate terrorist participants believed their actions were justified through the need to keep their girlfriends in line and "respectable".

Within the context of female control and violence, a gender minimisation narrative was apparent in this study. As discussed in Chapter Nine, the young women participants who described their deliberate use of violence viewed this behaviour as acceptable because they were women and their violence was therefore less consequential. Two female participants used this narrative and broader gendered 
beliefs as an excuse to use violence, as they felt it would not be taken seriously. In this sense, the female's violence was legitimised and minimised because of the common belief that it is men who are primarily the violent aggressors in relationships. This belief supports traditional gender stereotypes of women being meek and unable to exert control over males (Nowinski \& Bowen, 2012).

Another excuse used to justify and minimise dating violence in this study focused within some situational couple violence narratives was the labelling of these occurrences as "play". Within the situational couple violence context there was a strong narrative used to label violent and aggressive behaviours where young men and women reinterpreted violence as a "joke" or "just playing around". Even when injury occurred this was described as "over-zealous" play and "not intended" to cause harm.

Jealousy was a common recurrent motive in narratives for using violence and control for both young women and men in this study. Adams and Williams (2014) described jealousy permeating through young peoples' relationships and as an element in how they defined and experienced violence. Jealous actions included attempts to control who their partner spent time with, where they went and what activities they engaged in. This was also reported within the context of monitoring and tracking partners through the use of social media or other tools like Global Positioning System (GPS) tracking.

While jealousy was commonly displayed in young adults' narratives, the way it was enacted appeared different between situational couple violence and intimate terrorist relationships. Within situational couple violence relationships, jealousy was more likely to be normalised and reinterpreted as underpinning the individual perpetrator's commitment and care. Participants romanticised both young men and young women's presentation of jealousy as a representation of commitment, devotion and a deep level of care (Papp et al., 2017). Conversely, jealousy within intimate terrorist relationships appeared to be grounded in the young men's strong patriarchal and gendered beliefs. As described by Power et al. (2006) in their analysis of women's narratives of their recovery from IPV relationships, jealous and possessive behaviours were masked as positive signs in the early stages of the dating relationships and not as signals of danger, only later becoming associated with wider controlling and violent behaviours. 


\section{THE IMPACT OF VIOLENCE}

While there were minor differences in some factors that were investigated as possible reasons for gender findings in the experience of violence, there was a more obvious difference in reporting of injury between young men and women in this study. The impact of injury is central to the discussion of gender symmetry. Within the literature dating violence has been shown to produce fear, injury and emotional health consequences for both males and females, however these studies often show the negative impact to be far greater for females (Borsky et al., 2016). Chapter Nine highlights research on patterns of violence showing a more complex gendered picture, with women more likely to experience fear and terror from coercively controlling violence and far more likely to be physically injured and experience greater psychological harm as a result of male-perpetrated partner violence (Hamberger, 2005; Holtzworth-Munroe, 2005).

This was also seen in the current study where the survey results showed that it was far more likely for young women to report experiencing injury compared to young men. This was supported further through the interview analysis in Chapter Nine, where young women in an intimate terrorist relationship were more likely than young men to experience injury and were also at higher risk of experiencing injury compared to young men in situational couple violence relationships. Of the ten situational couple violence couples, five reported some level of injury, in three cases it was the young woman that was reported as experiencing injury compared to two young men (one in a same-sex relationship, one in a heterosexual relationship). For all of these participants the injury was minor, which is consistent with Johnson's (2008) theory that situational couple violence relationships largely experience less severe violence. Only one of the female participants in a situational couple violence relationship reported experiencing severe injury that required medical treatment. Conversely, of the six intimate terrorist couple relationships, all the young women experienced minor injury and two young women experienced severe injury in the dating relationship.

While there was injury experienced in 11 of the 16 relationships in this qualitative study, there was a strongly different tone to the narratives between situational couple violence and intimate terrorism couples when describing the fear and terror caused by the violence. While situational couple violence participants described being scared 
within particular situations, it was the distinct mention of the word "terror" by the young women who were victims in the intimate terrorism relationships that captured the additional impact of the violence that occurred within a stronger pattern of control. The experience of terror is the defining feature of relationships that have high levels of coercive controlling behaviours, which are designed to intimidate and frighten their intimate partner (Hamberger, 2005). In this study, participants' narratives of fear and terror appeared to be gendered, particularly in the context of intimate terrorism where male perpetrators used a range of controlling tactics including more severe physical violence. These findings were consistent with Dutton and Goodman (2005) who also found that the experience of terror was more commonly described in relationships exposed to high levels of coercive control.

Within situational couple violence relationships, female interview participants' feelings of fear were linked to the situation in which the violence occurred, but often this fear did not last once the episode had been resolved. This produced narratives, presented in Chapter Nine, where young women described feeling scared or fearful at the time the violence occurred or feeling trapped by continued monitoring and surveillance from their partner, but most did not describe ongoing fear of their violent partner, even when they decided to end the relationship. In contrast, the fear experienced by the young women in intimate terrorist relationships was described as an ongoing experience of control and violence. This was often due to the unpredictable nature of their boyfriend's violence that was frequently situated in a context of changing rules. This added terror and unpredictability appeared to make it much more dangerous for intimate terrorist victims to leave the relationship, as there was a greater perceived risk of harm. This type of fear and terror is what Hamberger (2005) describes as one of the defining characteristics of more severe and controlling violence - an ability to instil fear and terror in the victim, which is used as a way to control them by forcing them to conform to behaviours that will reduce the potential to need to use violence. When such patterns of control are present, a victim is not able to anticipate when controlling tactics will boil over into physical violence. This increases the uncertainty of their partner's actions, and can develop into forms of mental and emotional torture (Johnson, 2009). 
While this level of fear and terror was mostly reported in the context of young men's violence, both female and male participants within situational couple violence relationships described the negative impact of female violence towards their boyfriend and the psychological impact this had on young men as they worked to reconcile the disjoint between being male and being victimised. While this was not at the same level of fear as described by females, it does highlight the need to acknowledge and support young men who experience coercive and violent tactics at the hands of female partners. This view is supported by researchers such as Hines et al. (2007) who state that severe male victimisation has largely been ignored in the study of IPV, resulting in little understanding of the impact of extreme female violence on men. They concluded it was important not to discount male victims of intimate violence and that their experience is potentially serious, causes psychological and physical harm, and is valid in its own right.

\section{THE ROLE OF TECHNOLOGY AND SOCIAL MEDIA}

A theme that was common across all relationships in this study, but particularly in intimate terrorist relationships, was the use of technology and social media in the monitoring and surveillance of dating partners. Such tools and technology have extended the ability for dating violence to occur (Flood, 2019; Henry \& Powell, 2016). Technology and social media tools became mechanisms to support the enactment of men's control and they were able to use this technology for surveillance, a practice where people's actions and decision are controlled "by making them accountable to another who will scrutinise and criticise their actions" (Towns, 2009, p. 31).

The role of technology was also present in other aspects of enabling violence, in particular sexual coercion. Through the interviews two participants described scenarios where the use of media and technology facilitated their manipulation by their dating partners. These two participants, a young woman and a young man, described acts of consensual sex with their male partner within the realms of a normal 'healthy' relationship. However, both described the after effects of discovering that their partner had recorded their sexual activity on a mobile device and the later use of those recordings by their boyfriends to control and manipulate them. 


\section{CHAPTER SUMMARY}

In summary, the findings from this study support Johnson's typology with qualitative differences between situational couple violence and intimate terrorism. The narratives support the notion that there are different types of violence, and that dating violence is not a unitary phenomenon. The typology appears to reconcile instrumental violence embedded in the need to assert or restore power and control with violence that is more expressive (emotionally driven and based in conflict) and often perpetrated in a more dyadic scenario. Johnson's approach offers a critical means to create a narrative around the need to understand, validate and find solutions to violence that occurs in a range of different situations, with different contexts, drivers, and impacts.

Throughout the interviews there appeared to be traditional views of gender and dominant sexual scripts across all dating relationships with examples across the typology showing partners were expected to perform traditional gender roles, with women placed in a subordinate, often dependent, position. This appeared to have more negative impacts in relationships where hegemonic masculinity acted as a cultural norm and the dating relationships became the vehicle for young men to assert their status. This was predominantly evident in intimate terrorist relationships that were all perpetrated by men, all of whom exhibited destructive behaviours such as being violent, unable to control their temper, and holding strong gendered attitudes and beliefs. The distinct pattern of coercive control and the resulting terror seen in the gendered intimate terrorist category in this study was in strong contrast to the violence seen in situational couple violence. In the latter, the violence could cause injury and fear, particularly for female participants, but these participants also reported initiating dating violence; were motivated by elements of control and jealousy to gain their boyfriend's attention; and could cause psychological harm to their boyfriend.

This is where Johnson's typology creates the space to include discussion of women's use of violence, and this study supports the recognition that some women do use violence and this violence should not be ignored. Acknowledging both men's and women's use of violence allows for the creation of gender-specific interventions that account for dual roles as both victim and perpetrator, which are more likely to successfully promote behaviour change (Swan et al., 2008). 
There are some limitations with Johnson's typology that are detailed in Chapter Three, including: the exclusion of relationships with high levels of coercive control but where no physical violence was reported; utilising the CTS2 as a measure of violence; and whether there are distinct categories of violence or if these experiences are on a continuum along which couples may move over time. For these reasons I would be cautious about the wholesale application of Johnson's typology in practical terms, as doing so may risk misclassifying couples who are experiencing violence or inappropriately minimising and excusing behaviour in a category that is deemed less serious (Wangmann, 2011). However, Johnson's typology has clear merit, and provides a platform for a much needed conversation on the different types and experiences of violence that occur in intimate relationships, and the role gender plays in these (Flood, 2019).

The implications of these findings in addressing and preventing dating violence will be discussed in Chapter Eleven, which is the concluding chapter for this thesis. Here I will provide a reflection on my journey through the completion of this thesis, limitations of this study, opportunities for further research, and final thoughts and recommendations. 


\title{
ChAPTER ElEVEN - FinAl THOUGHTS, NEXT STEPS
}

\author{
What continues to trouble Lesley is that, despite she and Sophie being \\ concerned at Weatherston's behaviour, neither recognised he was displaying \\ classic signs of an abusive partner. Only after Sophie's death did Lesley \\ become aware of the signs they both missed. Her fervent wish is to find a way \\ where young women and their family and friends can recognise those signs... \\ What Lesley has come to understand herself is how a manipulative and \\ dangerous person like Weatherston can so easily get inside the head of a \\ highly intelligent young woman. (O'Brien, 2011)
}

It seems fitting to start the final chapter in the way the first chapter began, through the reflections of Lesley Elliott on the murder of her daughter Sophie Elliott by her exdating partner. The data in this thesis provided many examples of violence that were experienced across young adult dating relationships. These accounts of dating violence offer insight to the various ways that violence is enacted and how the current social context supports, justifies and normalises dating violence for some young people. The narratives also provided insights into a range of solutions and points of intervention that, if taken together and responded to, could help prevent the experience of dating violence and even save the lives of young adults navigating dating relationships.

In an attempt to provide solutions to address the prevalent, and sometimes devastating, experience of dating violence in New Zealand, I will use this final chapter to reflect on findings that supported existing theory alongside the limitations of the current study. I also provide concluding thoughts and recommendations built from this analysis. These recommendations can be considered in future policy design, development of educational resources and training, and investment in services and programmes to address and support young adults as they navigate into adulthood and build and maintain positive relationships, free from violence. 


\section{MY JOURNEY, GETTING TO THIS POINT}

It is fitting to reflect on my journey from when this thesis was first conceived to where I now find myself. As described in Chapter One, the main motivation for this thesis was to understand more about the role of gender within intimate partner relationships, particularly in the context of young adult dating relationships. To do this, my thesis set out to understand the influence of the current social context on the experience of dating and dating violence in New Zealand. I wanted to test the application of Johnson's (2008) typology of violence, which he developed to reconcile the range of findings on the role of gender in intimate relationship violence and to capture the complexity of IPV experiences.

I developed a three-year plan to complete my $\mathrm{PhD}$ while working full-time, but I was dreaming. A year on from enrolling my life changed when I fell pregnant and 2012 saw the arrival of my first child. This had implications on the progress of my thesis and was part of the reason I am only now finishing it. However, more significantly, the reality of having my own daughter made my research more important to me. What social context would my little girl grow up in? What would her experience be as a young adult establishing her own relationships? Was there any way that I could contribute to the understanding of young adults' experiences in dating relationships and support a future where she would be respected and safe in her dating journey?

When my daughter was only a couple of months old I continued the process of interviewing young adults as part of the qualitative component of this thesis. I was somewhat troubled by what I heard through the narratives that these young people shared. They described the normality of drinking and hooking-up, the risky situations they put themselves in, and the more traditional attitudes and beliefs that still existed amongst a more liberal narrative of binge drinking and casual sex.

I went back to work in 2013 and life continued to be busy. I moved into a leadership role and was no longer an analyst working in the IPV prevention space. My husband and I built a new house while living with my in-laws, then we had our second daughter, there were restructures at work and a move to a new agency... how much life could change. That did not stop me. My passion and commitment to this topic never waned, and in fact it intensified as my daughters grew. I could see the potential 
impact on my own girls, which fuelled my motivation to contribute to this area and support the future that I wanted for them.

I had also collected the views from sixteen young adults; their voices needed to be represented and shared. The time that had elapsed since I began my $\mathrm{PhD}$ journey to now provided me with a renewed focus on understanding this topic and what needed to be shared, which brings me to this point, a decade on from when I began.

\section{WHAT DID THIS STUDY CONFIRM?}

I have learnt a lot on this journey. There were a number of findings that confirmed my hypotheses and supported prior research in this space.

\section{DIFFERENT TYPES AND EXPERIENCES OF DATING VIOLENCE}

Firstly, in agreement with the large body of evidence detailed in Chapter Two (for example, Marganski \& Melander, 2018; Shorey, Meltzer, and Cornelius, 2010; Shorey et al., 2008; Harned, 2002; Jackson, 1998a), a significant number of young adults in this study experienced dating violence and a range of violent experiences that included many relationships with low-level violence. However, there were also some relationships where there was severe and terror-inducing dating violence.

Within this pattern of more severe violence it was more likely that traditional patterns of male dominance and control were present. This pattern of dating violence suggested that there was a diverse range of experiences of violence, control and ways that young adults maintain power and control over their partner. Applying Johnson's typology to this data allowed me to identify clusters of experiences of dating violence and to test whether these groups had patterns consistent with Johnson's analysis.

This study supports Johnson's findings (see Johnson, 2009a; Johnson \& Leone, 2005) that situational couple violence is more likely to be mutually perpetrated and less severe than violence categorised as intimate terrorism. Perpetrating more serious forms of violence was most often reported by young men and resulted in more negative impacts for young women including greater levels of psychological harm and physical injury. While some limitations in the development and application of Johnson's typology have been raised by academics and practitioners such as Wangmann (2011) and Meier (2015) (discussed in Chapter Three), this study 
confirmed, as hypothesised, that a model or typology such as Johnson's does allow for a narrative and insights into a wider range of experiences of violence situated in different contexts and which support a multi-dimensional response to preventing dating violence. This has been recognised elsewhere, with Flood (2019) concluding that there is considerable evidence that there are multiple types and experiences of violence and that Johnson provides one of the more developed approaches to conceptualising and understanding the different etiologies of IPV.

\section{INFLUENCE OF TECHNOLOGY AND MEDIA}

Secondly, the study confirmed the widespread influence of technology and media in the lives of young adults, which has been documented by many academics and researchers in this area (Manago et al., 2015; Lambert et al., 2012). The reach of modern technology and the different forms of media young adults interacted with daily was not in itself a surprising element of young adults' experiences; what I found surprising were the ways that young adults in this study described how ideals and expectations around gender, class and ethnicity were negotiated and performed through technology and media (Hutton et al., 2016; Towns \& Scott, 2008). As demonstrated by a number of researchers, media and technology play a significant role in shaping young adults' attitudes and beliefs, influencing their behaviours and desires to ensure that they maintain the right image and status amongst their peers (Hutton et al., 2016; Jackson \& Vares, 2015b; Manago et al., 2015).

One way this played out in this study was the need young adult participants felt to self-monitor and to modify and sanitise their behaviours and appearances to align with societal expectations of appropriate performance. This theme was presented in Chapter Seven and is also considered by Jackson and Vares (2015b) from their work with pre-teen girls. They discuss how young women participate in their own selfsurveillance to achieve an aesthetic that fits with wider societal expectations of appropriate performances of femininity.

A further finding in relationship to technology and social media was the representation of romantic relationships presenting harmful behaviours as normal in the context of love (Papp et al., 2017). In this context participants described recent celebrity stories in the media and how these stories sought to normalise and reinterpret aspects of male control and ownership over their female partner as being 
loving and chivalrous. In this sense, the media could function as a catalyst of misinformation that reinforced myths and beliefs supportive of men's ownership and use of violence (Breger, 2017).

Finally, another finding in relationship to technology and social media tools was how they became mechanisms supporting the enactment of men's control. Participants described how technology was used for surveillance, and for those in a relationship with a partner fitting the intimate terrorist category, the use of technology was part of a more systematic pattern of control. Some participants noted what is described by Henry and Powell (2016, p. 409) that: "one notable difference made by electronic communications and other technologies is that a stalking perpetrator can potentially have constant access to their victims, extending the feelings of exposure, vulnerability and fear of victims".

This study, like others, highlighted the ways that technology and media extended the ways that dating violence could occur (Flood, 2019). Any work to support young adults in dating interventions needs to leverage off the influence and role that the media and technology can play in a positive way (connection, forms of social movements and social good) and to minimise the negative impact it can have (misinformation, driving negative attitudes and beliefs). This is discussed later in this chapter when I explore the practice implications from the findings in this study.

\section{RELAXED VERSUS TRADITIONAL ATTITUDES, BELIEFS AND}

\section{BEHAVIOURS}

A third finding confirmed the inter-connection of engaging in a night-time economy, drinking to intoxication, hooking-up with people they had only just met and the normalisation and acceptance of sexually invasive behaviours such as unwanted touching, groping or kissing (Becker \& Tinkler, 2015). As discussed in Chapter Seven, most female participants described a level of acceptance of these types of sexualised behaviours and in some instances this behaviour was a signal to young women that they were both desirable and attractive to men. Flood (2019) states that, within heterosexual norms, the routine objectification of young women and the pressure they feel to accommodate the desires of young men leads to beliefs that sexual harassment is both expected and normal. While there was a strong narrative in this study about sexually invasive behaviours within social settings such as public 
bars and parties, a concerning narrative was around the interplay of alcohol and the hooking-up culture when this involved being taken to an unfamiliar private setting under the influence of alcohol, and engaging in sexual activity without necessarily any subsequent recollection of this interaction. Consistent with a review of the literature on hooking-up and drinking by Garcia et al. (2019), this study confirmed that the night-time economy and relaxed attitudes to casual sexual relations placed some young adults at greater risk of dating violence, particularly sexual assault, through no fault of their own.

There was a tension between the more relaxed attitudes towards intoxication and casual sexual relationships that young adults in this study described which appeared to be in contrast with their narrative on the enactment of sexual scripts and behaviours supportive of more traditional gender roles and reinforcement of dominant male culture (Hutton et al., 2016; Beres, 2014a). The burden of these competing belief systems fell significantly more on young women in what Farvid and Braun (2014) term as an enduring double standard where social norms make it acceptable for men to have casual sex, while for women casual sex is positioned as problematic and even dangerous. This sexual double standard can result in women being labelled as promiscuous when engaging in casual sex and should negative consequences result, they carry the blame for this (Beres \& Farvid, 2010). As described in Chapter Seven, on the one hand there were strong expectations for young women to be active participants in the night-time economy of drinking and hooking-up, but there was also a strong pressure for these same young women to maintain a level of decency. This is supported by researchers such as Hutton et al. (2016, pp.86) who posit that there is an expectation that young women engaging in the night-time economy must navigate the fine line between having fun while maintaining a level of "decency, desirability and respectability", behaving appropriately in order not to become unfeminine and undesirable. A theme emerged within the sexual scripts and gendered nature of dating relationships of young women using relationship status and engagement in sex as a proxy for their own desirability and self-worth. Jackson (2001) found a similar theme, where there was an inextricable link for women between love and sex, whereby sex represented the male wanting them and became a tool for their self-validation. These beliefs influenced some young women to stay in a relationship, even if it was harmful, because it was preferable to being single. 
For men, having sex was part of their performance of successful masculinity and heterosexuality (Farvid \& Braun, 2014, p. 124). Participants described how it was far more acceptable for young men to engage in casual sex and sex outside of a formal relationship, although some young men also discussed that there was still a need to maintain their own reputation with young women. They described needing to be viewed as a 'viable' dating option, carefully balancing an image of being a bloke and wanting sex against being seen by females as a player. To risk losing their dating credibility could jeopardise their ability to eventually engage in some form of more enduring long-term relationship, which is also a function of performing successful masculinities in later life (Chung, 2005).

\section{LIMITATIONS OF THIS STUDY}

This thesis does not come without its limitations. As with most research, there are constraints that need to be taken into consideration when interpreting and applying the findings. Five main limitations from this study should be considered.

Firstly, data from this study were collected through a convenience sample of selfselected young adult university and polytechnic students and crisis support agency clients. This means that the sample was not random. The lack of a random sample for the online survey was deemed acceptable as it was not a prevalence study but aimed to provide an understanding of the types of dating violence experienced by young adults in New Zealand and served to inform the qualitative study. However, a convenience sample does limit the generalisability of the results to the wider young adult population, therefore the analysis from this study should be understood within the context of the population that participated. The recruitment material that was produced may have further impacted the generalisability of the results in this study. The recruitment poster and website (Appendix 9) deliberately represented the study as one on dating and not on violence so that potential participants were not deterred by the prospect of discussing their experience of relationship violence. However, the posters themselves looked like a teen magazine and may have contributed to attracting a mainly student sample that experienced and described relationships embedded in the night-time economy. Future research needs to consider recruitment 
strategies and the tone and presentation of recruitment collateral to ensure it appeals to the range of participant required in the study.

A second limitation was the inability to measure differences in dating violence experiences between the student sample and the crisis support agency sample.

Recruitment of online survey participants was carried out by placing posters on notice boards around university and polytechnic campuses and posters given to crisis support agencies to circulate. However, when I asked survey participants how they found out about the survey, over two-thirds stated that they found out about the survey through 'word of mouth', where they got information about the survey through a friend's Facebook post or through key informants who connected agency clients into the survey. I did not anticipate this mode of recruitment. As a result, the exact number of survey participants in the 'crisis support sample' could not be calculated due to the limited way in which the survey question asked how participants found out about the survey. Respondents could either reply "through a poster on campus" (49.2\%), "through a poster at an agency" (12.8\%), or "through word of mouth" $(38.0 \%)$. Numbers recruited through "word of mouth" are likely to include those who were recruited through key informants, even if they were agency clients, as well as participants who found out about the survey from other participants (e.g., through Facebook). As a result, analysis could not be meaningfully carried out by sample type as the variable was not an accurate measure of whether or not young adults had been involved with crisis support agencies. However, the main rationale for including crisis support clients in this study was to ensure survey respondents represented a range of experiences of violence from minor to severe. This was confirmed by the analysis in Chapter Five. This study also provided support for designing recruitment techniques that leverage off the use of social media and technology, particularly when recruiting young adults.

The third limitation of this study is connected to the self-report nature of the collected data. Participants in both the online survey and interviews were asked about their own experiences in dating relationships, and to report on their partner's use of control and violence. The self-report nature of this data relied on the respondents recalling and reporting different acts of violence across a 12-month period, both their own violent acts and reporting violent acts they recalled their partner committing. Research that 
has interviewed both partners in a relationship has found that there can be significant disagreement about the occurrence of violence: when it began, who initiated it and the impact of the violence (McHugh et al., 2005).

Research has also found that there are differences in the way that men and women report on experiences of violence, which is summarised by Lehrner and Allen (2018, p. 1702): “in sum, women's versus men's IPV were reported to have different social meanings, communicate different threats, provoke different peer responses, and have different consequences for those involved". Currie (1998) found that both women and men under-reported men's violence towards women while men were more likely to overstate women's violence towards them. The reasons for this pattern of reporting is complex. One reason posited in Chapter Two is that men's violence is far less acceptable than women's violence, therefore men may be less likely to report being violent due to the stigma of doing so, or due to beliefs that their behaviour is justifiable (Shorey et al., 2008). Women may also be more likely to report their use of violence in an intimate relationship because women's violence is viewed by society as less common and less serious and they are less likely to be condemned for its use. Women are also more likely to view their own aggression as violence and may recall such acts more readily than men as it is against the norm for women (Lewis et al., 2002). Another reason why there may be differences in reporting of violence is that women who are victims of men's aggression can normalise it by rationalising that it shows their partner loves them, or by blaming themselves for their partner's use of violence. These attitudes make it harder for women to reject violent men (Kimmel, 2002).

Options to reconcile relationship violence findings, including interviewing both partners, come with their own issues and limitations including the need for careful ethical consideration, particularly in intimate terrorist-style relationships where no interaction between partners should be facilitated that could put the victim in harm's way or cause additional psychological distress (Migliaccio, 2001). Therefore, while there may be inconsistencies in what is self-reported by males and females in this study, there is no empirical evidence that other methods of collecting data on the experience of IPV are more accurate in reporting it (Straus \& Gozjolko, 2014). 
Therefore, this was a valid approach for a study that looked at patterns of violence and did not seek to establish prevalence rates for dating violence in New Zealand.

The fourth limitation of the study was the use of the CTS2 (Straus et al., 1996) to measure the experience of dating violence reported in the online survey. As discussed in detail in Chapter Two, the CTS2 has been criticised for its inability to report the context of violence and for its simplicity in classifying how someone is violent, or not (for example see Barnett et al., 2005; Hamberger 2005, Sugarman \& Hotaling, 1989). Gradations of violence are treated as equivalent; for instance, a respondent who indicates that they 'shouted at their partner' is considered the same as a respondent who has reported using a range of frequent and severe tactics on the scale. However, the CTS2 is widely used across the dating violence literature so using it in this study allows for comparisons with findings in other jurisdictions. More importantly, the CTS2 was used by Johnson to develop and refine his typology (Johnson \& Leone, 2005). The CTS2 was therefore the optimal tool for collecting victimisation and perpetration data in order to replicate his typology. It is also important to note that the lack of contextual information collected through the CTS2 was mediated in this study through the qualitative interviews. The interviews were specifically focused on testing Johnson's typology so provided for significant analysis and discussion on the context, motives and impacts of injury. However, further studies on dating violence and utilising a typology such as Johnson's should consider applying definitions, tools and methods that may provide findings that are more sensitive in how they define and contextualise aspects of the study of intimate partner violence and limit the likelihood of reinforcing unhelpful classifications and conceptions of violence.

The final limitation to the study was the small numbers of survey participants across some of the four-category classifications. The survey sample had a high proportion of participants and partners who were classified as situational couple violence, with fewer in the intimate terrorism category and very few classified as mutual violence and violent resistance. The statistical implications for this were discussed in Chapter Six, with analysis of the sub-groups unable to be carried out due to the potential of participants being identified when there were fewer than 5 in a category. However, the survey data were used to form the basis of the qualitative inquiry where the classification was used to select interview participants and to test the validity of 
Johnson's typology, maintaining the rationale for classifying participants into these categories. It does raise the question on the use of the statistical methods in future studies that aim to understand the meanings and complexities of violence in dating relationships and whether qualitative inquiry-alone could achieve this. The strength and richness of the qualitative data in this thesis suggests that this is feasible and powerful in its own right.

\section{STRENGTHS OF THE CURRENT RESEARCH}

While there were limitations in this research, there were also a number of strengths that are worth noting, the most significant being the collection of data that distinguished between the types of violence experienced by young adults in dating relationships.

My conclusion from the literature review supported a need to apply a framework that was more nuanced and sophisticated in explaining the complexity of violence in dating relationships and to move beyond seeking a singular explanation to the contradictory findings of the role of gender. Following my discovery of Johnson's (2008) typology, I felt that there was merit in using this to reconcile the differences between studies that found gender symmetry and those that did not. Therefore, this study was specifically designed to measure concepts and elements necessary to replicate Johnson's typology and to test its applicability and relevance in the New Zealand dating context. This was the first time this approach had been applied to young adult dating relationships in New Zealand. In taking this focus I was also able to establish what dating meant to young adult participants in New Zealand and how this was constructed within their current social context. In other words, I could better understand the role of the New Zealand context in shaping the experiences of dating for the young adult that took part in this study, as they moved into more serious relationships and were potentially more at risk of experiencing the negative impacts of violence.

The study was strengthened by widening the sample beyond the student population to include participants sourced from crisis support agencies. This ensured the recruitment of a broader range of experiences of dating across the violence continuum from less severe to more severe and allowed data that represented the experiences of 
young adults who sought help and support for relationship violence to be captured. This was important as research based on national surveys, community samples and general population studies tend to produce different rates and constructs of IPV than studies using shelter samples, perpetrator treatment participants, hospital patients or those seeking help (Holtzworth-Munroe, 2005). Having different sample frames in this study ensured that a wider range of dating violence experiences was represented.

A further strength of this study was including the experiences of both males and females. As a result I was able to understand the experiences and complexities of dating and relationships from both the perspective of female and male participants. One of the limitations of Johnson's work is that it is based on married women's accounts of IPV, thus we know little about men's reported experiences of violence in his typological context. Acknowledging the experiences of both genders means different factors can be understood in the aetiology of dating violence (Ortiz et al., 2015). This is particularly important as there were a number of male participants who reported being victims of female violence (whether mutually or bi-directionally). It is important then that factors for female-perpetrated violence are researched and better understood to be able to address and prevent all types of dating violence (Espinoza \& Warner, 2016).

The final strength of this study was the use of a mixed methods approach which allowed the contribution of different methods to come together and extend the understanding of an important social issue. In doing so, I recognised the strengths and limitations of different methods and the value in combining these approaches to tell a more comprehensive story of the experience of dating and violence in New Zealand. Survey data were used to understand the amount of violence experienced by respondents and to look at patterns in these experiences from an anonymised position, while qualitative interviews were used to understand the complexity of these experiences. This allowed rich narratives on the intricate interplay of young adults, their social context, dating, and violence to be captured.

\section{FUTURE RESEARCH AREAS AND POSSIBILITIES}

In this thesis I replicated Johnson's typology of violence and tested the validity of the statistically derived categories through qualitative investigation. While investigating 
these research questions there were also a number of areas that emerged that I will highlight as opportunities for future research.

The first opportunity for future research is to extend the analysis of Johnson's typology to see if his model would be more useful if it included coercive control that occurs in the absence of physical violence. While Johnson (for example, see Kelly \& Johnson, 2008) talks about the negative effects of coercive control in the absence of physical violence, the sample inclusion criteria for his typology only included couples where there was physical violence present. To allow a more direct comparison with Johnson's findings this study maintained his analysis protocol, which was to only classify participants who reported physical violence against their level of coercive control. However, it seems critical to expand such a framework beyond physical violence when there is such a strong case for the detrimental effects of coercive control in the absence of physical violence (Anderson, 2008).

A second future research opportunity is to expand the quantitative analysis to look at the difference in risk factors and other characteristics of New Zealand young adults within Johnson's typology. This would provide more understanding of the risks, motivations and attitudes and beliefs that might differ between categories, further enhancing the typology. This would also strengthen the development of different approaches to intervention and prevention support that could address the different drivers and impacts of the range of violence young people experience.

While not deliberately excluded, young adults in same-sex relationships were not a specific focus of this research. However, the impact of dating violence and other forms of IPV disproportionally impact members of marginalised groups including ethnic and gender minority groups (Beres, 2017). Research regarding dating violence within the LGBTQI (Lesbian, Gay, Bisexual, Transsexual, Queer, Intersex) communities is growing but still has a way to go in understanding the specific scale and nature, unique attitudes, beliefs, inequalities and risk factors that support or endorse such violence. Where studies have been conducted, findings show that there are high rates of dating violence within the LGBTQI community. A study by Freedner et al. (2002) found that bisexual men and bisexual women were more likely to report sexual abuse and were more likely to report fearing for their safety, compared to heterosexuals. These findings were supported in a more recent study in the USA 
where significantly higher rates of dating violence were experienced by LGB youth, both as victims and perpetrators (Dank et al., 2014).

Likewise, there is limited research in New Zealand on Māori and other ethnic minorities in dating relationships, and more broadly in IPV (Eruera \& Dobbs, 2010). Prevalence data show that Māori are over-represented in statistics on victimisation and perpetration of IPV and other family violence statistics. This was demonstrated in the 2018 New Zealand Crime and Victims Survey, which reported that Māori were more likely than other ethnic groups to experience IPV and/or sexual violence over their lifetime. For example, 23\% of Māori respondents reported experiencing IPV compare to $16 \%$ of NZ European respondents and 15\% of Pacific People (Ministry of Justice, 2018). However we know little about the relevance of Western models and frameworks in capturing the experience of violence across cultures, with Eruera and Dobbs (2010, p. 7) stating that Māori academics and sector professionals "argue that models of analysis and intervention methodologies based on Western or mainstream thinking have been consistently ineffective for Māori".

There is an urgent need to understand dating and violence across a wide range of groups and to focus on kaupapa ${ }^{7}$ Māori and culturally-specific research on the context, impacts and solutions to prevent and support young Māori engaging in dating relationships (Eruera \& Dobbs, 2010; Beres, 2017).

Finally, there is an opportunity to conduct further qualitative research that contextualises violence within the national culture, and seeks ways to influence and educate young adults. Young adults are critical players in the design of interventions and prevention around dating violence, if for no other reason than the fact that the landscape is moving fast in areas such as the reach, impact and use of technology (Flood, 2019; Henry \& Powell, 2016). The quick pace of technology change can leave traditional policy formation and service design out of touch with the current social context and the ways that young adults interact within it.

7 "Kaupapa Māori research differentiates itself from other research in that it is grounded in Màori philosophy and knowledge and may use methodologies which reflect customary practices, values and beliefs, subjective approaches rather than objective" (Eruera \& Dobbs, 2010, p. 25). 
The research opportunities identified here will help to further build the understanding and focus needed to more fully address and support young adults who experience dating violence and to extend this foundational research to ensure that we give this issue the attention that it deserves. This is especially important given the high rates of violence experienced, the social context within which this violence takes place, and the limited specific New Zealand-based interventions to support young adults into positive healthy relationships.

\section{PRACTICE IMPLICATIONS}

The current study's analysis confirmed that dating violence is widely experienced and is a significant issue that needs to be addressed as it can have long lasting impacts for young adults, now and in their future relationships. Negative consequences include mental health issues such as anxiety and depression; the adoption of negative coping strategies such as substance abuse; and physical impacts including injury (Shorey et al., 2008). It is therefore critical that effective strategies are developed to prevent and intervene in such violence (Peterson, et al., 2018). This section discusses some existing and emerging areas of focus in addressing and preventing dating violence in young adults. This is not a comprehensive review of effective interventions but focuses on areas that align with findings from this study.

One area that the study findings promote is the need for interventions that provide a multi-faceted, sophisticated response to different types of violence. There is no one solution (Flood, 2019). Recognising this diversity in the aetiology of dating violence allows us to challenge and expand how we prevent and intervene and to develop a range of responses that meet the needs of people experiencing different types of dating violence (Wangmann, 2011). For example, within dating relationships where there is situational couple violence it may be more effective to support partners through joint programmes that address conflict resolution and equality. However, for intimate terrorist relationships, where there is coercive control and terror, separate responses are likely required for the perpetrators, who need to be held accountable for their actions, and for victims who may need support to leave a violent relationship and to ensure their safety (Johnson, 2014). 
One approach is to develop a differential response by translating Johnson's typology into a prevention approach - while this may have some merit (Johnson, 2014), there are limitations. As outlined in Chapter Three, one of the main risks is the misclassification of couples that may result in the system responding in a way that could increase the risk of harm to the victim (Wangmann, 2011). Johnson himself is cautious about using his typology as a classification tool and raises the implications of making incorrect classifications, stating: "it is probably wise to assume that all violence is intimate terrorism until proven otherwise" (Johnson 2008, p. 82). Alongside the risk of misclassification is the risk of reinforcing minimising attitudes towards violence in intimate relationships (Wangmann, 2011; Pence \& Dasgupta, 2006). For example, there could be a perception that situational couple violence is common and perceived as less severe by the justice system, thus reinforcing myths that it is not that serious, it was just a momentary loss of control and men and women are equally violent.

What we can take from Johnson's typology is the importance of viewing IPV as a complex phenomenon, which supports approaches to prevention and intervention that deviate from one-size-fits-all (Flood, 2019).

\section{PREVENTION APPROACHES}

While the nature of dating violence and other IPV is complex, there is increased consensus that risk factors for perpetration and victimisation can be modified or eliminated (Vic Health, 2007). Therefore prevention has become an important focus over the past two decades alongside responses to victims and perpetrators where violence is already occurring (Flood, 2019). In particular, the public health approach has provides a framework that classifies prevention activities based on the violence continuum. In other words, prevention focuses on when violence occurs in a relationship - either before the violence starts, once violence has begun, or after violence has occurred (Flood, 2019). These actions are termed as - primary, secondary and tertiary prevention.

Primary prevention focuses on preventing violence before it begins (Flood, 2019) and can include activities that address broadly held attitudes, beliefs and cultural factors that support and condone violent behaviour, often targeted to whole of population. Secondary prevention focuses on identification and intervention, aiming to reverse the 
likelihood of potential victimisation and perpetration (Flood, 2019) and to minimise the impact of the violence and prevent further harm (Beres et al., 2019). Tertiary responses are most likely to include crisis support, counselling and advocacy for victims, and criminal justice responses and counselling support for perpetrators (Flood, 2019). This public health approach is underpinned by its focus on evidencebased policy and programme design and addressing issues from a comprehensive multi-level perspective that includes understanding the contributions of the social context within which relationships and violence occur, and ensuring a strength-based lens is applied (Flood, 2019; Beres, 2017).

The public health approach has been increasingly utilised as a frame for prevention, as it captures the complex interplay of risk and protective factors at an individual level, alongside familial and relationship factors, community factors and societal factors (Vic Health, 2007). This is the 'ecological model' developed and documented by Bronfenbrenner (1979). His model was extended by Belsky (1980) who applied the ecological model to child maltreatment, and more recently it has been adapted by Heise (1998, p. 263) "for conceptualizng the aetiology of gender-based violence”. The benefits of adopting an ecological model are that it draws on and integrates a range of disciplines to account for the complexity of factors associated with intimate violence. It emphasises the requirement for multi-level approaches to prevention and acknowledges the need to support change across the levels (Gibbs, et al., 2020; Vic Health, 2007).

While the ecological model has been widely adopted, Flood (2019) outlines some limitations in its application to prevention efforts, namely:

- the focus on some prevention efforts still emphasises individual-level factors;

- the focus on attitudes risks neglecting other factors that shape men's violence including structural and institutional inequalities;

- it neglects the influence of other actors including the role of institutions and governments; and,

- that it does not always account for the complex impact of social order.

Similarly, Gibbs et al. (2020, p. 9) stress the limitations of the ecological model in their statement that: "the ecological model provides an important framework to 
understand the multi-level nature of IPV, but does not adequately capture the interconnections between different levels, nor provide clarity about which risk-factors may be indicators of broad underlying latent constructs". The ecological model cannot then predict which measures will be successful; conversely, some effective interventions will address multiple drivers of violence but only at one level of the ecological model. However, Flood (2019) concludes: "nevertheless, the ecological model has offered a valuable, feminist-informed, and mobilising framework for understanding men's violence against women and its prevention” (p. 48).

A public health approach means that the prevention of dating, and other interpersonal violence, requires the simultaneous provision of services and support that can respond to the wider range of experiences of young adults in relationships - from addressing where violence has already occurred to a focus on prevention approaches before dating violence starts (Flood, 2019).

\section{Prevention in TERTIARY SETTINGS}

This thesis highlighted the changing context of dating in New Zealand, and explored linkages to the night-time economy of hooking-up and drinking to intoxication. As discussed in Chapter Two, since the early 1980s, when the number of studies looking at violence in dating relationships grew, there has been a rapidly changing social context. Young adults have more fluid paths into adulthood and there has been a rapid decline of traditional dating in the Western world leading to a growing acceptance of 'hooking-up' and casual sexual encounters often within the situational context of going out on the town and consuming excessive amounts of alcohol (Hamilton \& Armstrong, 2010). Given this, it is likely that contemporary young adults are at increased risk of experiencing physical and sexual violence (Noel et al., 2016). What was evident from this thesis was that engagement in hooking-up and the night-time economy was often, but not exclusively, linked to aspects of the student lifestyle. As previously detailed in Chapter Two, a significant body of dating violence literature has been conducted with college students, or in universities, that show high rates of interpersonal violence (for example, Marganski \& Melander, 2018; Shorey et al, 2010; Makepeace, 1981). More so, since the 1980s there has been a strong focus on sexual victimisation with growing recognition of women's experience in the university setting. Fisher et al. (2010) discuss how sexual violence is a complex issue 
to present as it is "embedded in routine activities and lifestyles that are common among many college students" (p. 179). Over recent decades there has been an increased response by universities towards relationship violence, particularly sexual violence, on campus - "prevention efforts take a multitude of forms, and many campuses implement multiple approaches as they seek to maximize positive change in their campus community” (Banyard et al., 2018, p. 145).

In the USA, campus prevention of sexual violence has been supported through two key pieces of legislation. The first piece of legislation was the Jeanne Clery Disclosure of Campus Security Policy and Campus Crime Statistics Act ${ }^{8}$ in 1990, which mandated reporting of any campus security incidents (Perkins \& Warner, 2017). The Act was amended in 1992 adding specific requirements for the development and implementation of prevention and intervention approaches to address sexual violence across colleges and universities (Perkins \& Warner, 2017). The legislation was further updated in 2013 with the Campus Sexual Violence Elimination (SaVE) Act, which required security incidents to include dating and domestic violence and stalking. The second major US legislation is Title IX. Initially passed in 1972, Title IX was originally developed to require education programmes to provide equal opportunities for men and women. It was extended to address gender equality across ten key areas, including sexual harassment, which "means to protect all students from unwanted sexual behavior on campus that could impede their opportunity to obtain an education" (Perkins \& Warner, 2017, p. 239).

Within this legislative context several barriers to reducing sexual violence on campus have been identified. These include definitional issues around sexual violence and consent; negative unintended consequences from mandatory reporting; focusing on victims and not including others in the ecological context (e.g., accused student, staff); and a lack of evidence in the campus setting of effective policy to prevent sexual violence (Perkins \& Warner, 2017).

Fisher et al. (2010) described the diverse range of university prevention efforts falling under three categories - the need to change the traditional culture on campus, the

${ }^{8}$ Originally titled The Crime Awareness and Campus Security Act 1990. 
protection of female students against sexual predators, and providing help and support to victims of sexual violence. However, Fisher et al. (2010, p. 183) stress that: "the critical issue is whether these initiatives "work" to diminish the incidence of female sexual victimization". In other words, with the heightened focus on preventing increases in sexual violence across campus is the acknowledgment that "no single approach to sexual violence will solve the problem and instead that integrated approaches are required that engage all members of the campus community and span across policy, support and prevention activities” (Beres et al., 2019, p. 647). Researchers such as Berkowitz (2004) discuss the development of comprehensive approaches to the prevention of violence that include different members of the system or community in a coordinated and collaborative manner.

An example of where such an integrated, coordinated approach has been applied is the University of Otago in New Zealand, where a whole-of-campus programme began development in 2016, with implementation in 2018. The university adopted an evidenced-based approach and engaged all members of the university community, including staff and students, in the prevention of sexual violence and to support those impacted by sexual violence. This comprehensive approach is the first of its kind internationally to be implemented and included a response at a primary, secondary and tertiary level including prevention activities, support and advocacy, education, social marketing and investigation (Beres et al., 2019). Beres et al. (2019) conclude that this evidence-based model is "only the first step to addressing sexual violence on campus" with a comprehensive evaluation programme needed to "monitor, adjust and improve the response to sexual violence" (p. 659).

Other universities around the world, including the UK and Australia, have also increased attention on the issue of sexual violence - conducting campus surveys measuring the prevalence of sexual violence, and developing policies and guidelines to respond to this violence (Beres et al., 2019).

More recently, consent-focused prevention approaches have emerged across many universities which focus on educating young people on how consent is defined and how to communicate consent (Beres, 2020), moving prevention "from centering around a message of 'no means no' toward a message of 'get consent',' (Beres, 2014b, p. 373). However, in a recent study by Beres (2020), some educators and 
activists raised concerns over the ability to change rates of sexual violence with this approach, although they did see potential if there was a theory of change as a foundation for the design of such interventions.

Their [educators] perceptions of the potential for consent education to create change are complex. Educators simultaneously see transformative potential in consent education while maintaining scepticism about the possibility of consent education to change perpetrating behaviour. (Beres, 2020, p. 227)

This increased attention to the issue of dating violence in tertiary education settings is promising, but more work is needed to ensure programmes are evidenced-based, culturally appropriate and are evaluated to assess their effectiveness in reducing violence (Beres, 2020; Flood, 2019). The findings from university-based studies are also limited as they do not provide a framework for prevention that can be used for young people outside of university settings. This shows the importance of reinforcing and extending programmes that are delivered in schools. As Beres (2017) writes, it is unlikely that dating violence emerges spontaneously in adolescence, and likewise in young adulthood, therefore supporting the need to deliver programmes at both primary school and secondary school levels to prevent bullying and build expectations around healthy friendships. Jackson's (1998a) conclusion on prevention in the context of teen dating violence and harmful media messages is that the secondary school setting is ideal for developing critical analysis of the media - "the high school years are eminently suitable for the introduction of wide-scale violence prevention programmes, particularly the prevention of dating abuse” (Jackson, 1998b, p. 35).

However internationally there is a lack of well-evaluated primary prevention programmes and interventions - and where there is evaluation this is often limited both conceptually and methodologically (Flood, 2019; Ellsberg, et al., 2015). However, Fanslow (2005) states that where there has been robust evaluation of dating violence prevention programmes there is evidence that some of these responses do produce positive changes in attitudes towards dating violence and also a reduction in victimisation and perpetration of dating violence that is sustained over time. For example, Foshee et al.'s (2004) evaluation of a USA-based initiative called Safe Dates Program found school-based sessions and other student-engagement activities provided promising findings in the prevention of dating violence. 
Where prevention programmes have been evaluated and demonstrate promising findings, some common elements emerge which are outlined by Beres (2017) and Flood (2019). These include taking a strength-based approach; working across the school, home and community system; running the programme for sufficient hours, and groups-based programmes, including interactive components so that participants can test out ideas and try new behaviours through role play for example. Also key to success is having skilled facilitators that are trained to deliver prevention programmes.

Other interventions that have produced evidence of effectiveness within the secondary and tertiary education sector include programmes that teach about consent and ethical sexual relationships (Carmody \& Ovenden, 2013), rape education sessions (Brecklin \& Firde, 2001); bystander intervention (Coker, et al., 2017), and self-defence courses (Jordan \& Mossman, 2019).

However, the prevention of dating violence cannot be fully addressed in education settings. Studies have shown that IPV and dating violence is more likely to occur when young men and women are socially isolated or disengaged, including not attending education institutions (Flood, 2019). Therefore prevention in the education setting need to be developed and delivered alongside community, organisational and government responses - where they collectively address the multiple drivers of dating violence (Flood, 2019).

Concurrent with prevention efforts there needs to be a continued focus on responding when violence has occurred. This should reflect trauma informed policy and practice. While prevention is critical to stop violence from occurring in the first place a range of interventions is needed that draw from a strong evidence-base and are supported with investment in evaluation to test their effectiveness in the context in which they are delivered.

\section{AdDRESSING ATTITUDES AND BELIEFS}

A further area of focus in the prevention of dating violence involves large-scale social change through addressing attitudes and beliefs towards traditional gender roles (Flood, 2019), particularly those that underpin the use of violence in dating relationships. Findings from this thesis confirmed that, on the one hand, young adults 
are navigating through a somewhat contradictory landscape including understanding their position within more relaxed attitudes to relationships, casual sex and drinking, while on the other needing to adhere to more traditional expectations about gendered roles in relationships. The intersection of these contrasting cultures and norms creates the space for dating violence to occur in a number of ways and signals the need to develop programmes that aim to shift gendered norms and empower young women (in particular) to seek equality and independence (Beres, 2017).

As Gulliver and Fanslow (2016) write, one way to address and reinforce changes in attitudes and behaviours that support the use of violence in intimate relationships is through gender transformative programmes. Flood (2019, p. 389) supports this view and the need for the transformation of gender inequalities to occur at every level of society to "build just and respectful gender relations in relationships, families, and communities”. Beres $(2017$, p. 7) also talks about the need to develop gendertransformative prevention approaches that challenge dominant masculinities and "provide alternative norms about both masculinity and femininity that value nonviolence and non-aggression in boys while allowing for vulnerability". Likewise, Jewkes et al. (2015, p. 1580) discuss the value of prevention approaches that "transform the relations, norms, and systems that sustain gender inequality and violence”. The effectiveness of intervention programmes that focus on gender transformation, which promote gender equality and transform gender norms, are shown to be effective for both men and women (Jewkes et al., 2015). What the quote above highlights is the need to change gendered norms and make the structural changes required to reduce and eliminate gender equalities. As Baker (2013) writes, how gender roles and masculinity are displayed have been clearly linked to levels of violence against women. This thesis highlighted the strong presence of traditional gendered norms and stereotyped constructions of masculinity and femininity in the context of dating relationships where young men were viewed as strong and dominant, entitled to make decisions and employ aggression and control to limit the independence of women. In contrast, women were often expected to be compliant, polite and proper (Barnett et al., 2017). As discussed in Chapter One, it is the lower social status ascribed to feminine norms that are particularly negative and harmful to women (Our Watch, 2017). Addressing such gendered positioning needs to occur in the context of reducing structural inequalities and eliminating the gendered 
hierarchies of power that position men as superior and privileged (NZFVDRC, 2020). This needs to be addressed through seeking economic and social parity for women and promoting their independence and capacity for decision-making. Thus the need to challenge gendered norms and stereotypes, creating a context that endorses positive respectful relationships, promotes the independence and decision-making of women, and challenges the harmful attitudes and beliefs that condone the violence against women (Our Watch, 2017).

A growing body of literature argues that reduction in gendered violence can occur by engaging men in its prevention (Baker, 2013; Kimball et al., 2013; Tolman, Casey, Carlson, Allen, \& Leek, 2019). Michael Flood, a leading expert writer on engaging men and boys, lists the three key elements for doing this: 1) recognising that most violence again women is perpetrated by men; 2) that violence against women is shaped through constructions of masculinity; and, 3) that there is a positive role men can play in the prevention of violence against women (Flood, 2019).

Engaging men in prevention shifts their involvement to being partners in prevention, supporting efforts to change norms and attitudes that justify and endorse violence against women - "work with men to prevent violence against women is emerging as a robust and identifiable global movement” (Kimball et al., 2013, p. 925).

\section{AdDRESSing FEMALES' USE OF VIOLENCE}

A further area to consider based on findings from this thesis is that both young men and women reported using and experiencing violence, and in many relationships the young adult was both a victim and perpetrator of violence. As described in Chapter Two, researchers such as Hines et al. (2007) state that severe male victimisation has largely been ignored in the study of IPV, resulting in little understanding of the impact of extreme female violence on men. Migliaccio (2001) also reported from his research that when men tried to access support services they were met with prejudice and demoralising reactions. These examples suggest that a cultural shift is required to recognise female-perpetrated violence as a problem that needs to be addressed. Throughout the narratives in this study, and in the literature review, there was evidence that women's violence was often normalised, minimised and even condoned (Nybergh et al., 2016; Nowinski \& Bowen, 2012). This was coupled with societal 
beliefs that men cannot be victims without diminishing their successful attainment of masculinity:

... these [male] victims are seen as challenging and contradicting the status quo, and the gender expectations and social ideals of men. Men are not expected to be victims, vulnerable, hurt, damaged, emotional and sensitive; by enacting these traits, however, they are not achieving hegemonic masculinity and are not seen as 'real' men. (Javaid, 2018, p. 200)

Therefore it is important that interventions and education programmes include the occurrence of women's perpetration and men's victimisation so as not to deny the education and assistance that some young women require to stop their use of violence and to offer the support that young male victims of violence require - as Flood emphasises, "efforts to address these forms of violence are vital as well" (Flood, 2019, p. 13). An important element is that young women who reported perpetrating dating violence also frequently reported being victims of dating violence. This has implications for education and prevention strategies that include both young women and young men, including the need to ensure safety is paramount for all participants while acknowledging women's greater risks of being harmed overall (Swan et al., 2008). As Swan et al. (2008) report, interventions that are gender-specific and account for dual roles as a victim and perpetrator are more likely to successfully promote behaviour change, in some circumstances.

Recognising and responding to women's perpetration need not minimise the more frequently harmful gendered violence that women experience in intimate relationships, but acknowledges that men are often victims of various forms of violence, including that perpetrated by other men as well as by intimate partners (Flood, 2006).

\section{THE ROLE OF MEDIA AND TECHNOLOGY}

Finally, the role of media and technology was strongly reflected in participants' narratives of peer and relationship interactions. This was supported by research such as Baker and Carreno (2016) who found that technology was widely used in the establishment of ongoing communication in relationships, even in ending a relationship. There is a need to leverage the influence and reach of social media and 
technology in positive ways to help reduce dating violence and to avoid reinforcing attitudes and beliefs that are implicit in the use of violence or that minimise and glamorise violence and control.

With a generation developing sexual scripts that are informed by a medium that portrays a view of sexuality that is often shallow, misogynistic, patriarchal, and that almost invariably occurs in a context separated from healthy, committed relationships, there may be attitudinal and behavioral consequences that are difficult to undo. As successive generations grow up immersed in pornography, it may become increasingly important to supplement their learning about sex with views of sexual relationships that go beyond the lessons taught in pornography. (Braithwaite et al., 2015, p. 121)

As Flood (2019) writes, and what this study highlighted, the growth of technology and media has extended the ways that dating violence can occur (Flood, 2019). In exploring sexual violence in the digital age, Henry and Powell (2016, p. 398) outline the "phenomenon of technology-facilitated sexual violence (TFSV), where mobile and online technologies are used as tools to blackmail, control, coerce, harass, humiliate, objectify or violate another person”. In this context, Baker and Carreno (2016) discuss the need to educate adolescents and young people around warning signs of early behaviours that can escalate to more harmful abusive behaviours including monitoring and surveillance.

It is also necessary to educate young people to critically analyse what they are viewing online and through social media and popular culture (Jackson, 2015b). This critical assessment also needs to acknowledge the role of pornography, which is now highly accessible and has become an unintentional learning tool for adolescents and young adults (Lambert et al., 2012). The Classification Office in New Zealand confirmed this in their recent report talking with youth aged 14-17 about their experiences with pornography. They reported that both young men and women accessed pornographic material for sexual pleasure, through curiosity, and to explore their sexuality but they were also often unintentionally being 'educated' on sexuality (Classification Office, 2020). One theme arising from this study was that young people felt that they had to navigate their exposure to pornography on their own. The study emphasised including porn literacy in healthy relationships and sexuality 
education, and making information available in different places and in a range of ways for young people to interact with. This acknowledges the complex and dynamic approaches needed to educate young people on healthy sexual development and to support young people to apply a critical lens to viewing pornography.

To address and prevent online harm there needs to be a counter narrative to the unhelpful and harmful messaging young people are exposed to through social media, the internet and increased access to pornographic material (Keene, 2019). Jackson (2001, p. 318) writes in reference to the powerful romantic narrative of promised perfect relationships: "our educative work will be more productive and more likely to succeed if it does not negate romantic narratives but explores the limitations, dilemmas and problematic aspects while highlighting alternative, more empowering cultural narratives". As such, we need to educate young adults that what they view online may present unrealistic, gendered and sometimes hostile images and narratives of relationships and sex, and provide alternative healthy views and expectations around hooking-up and dating (Keene, 2019).

More generally, there is a need to develop strategies to keep young adults safe and encourage ethical practices online and through the use of technology. As Powell et al. (2020) write from their survey of 5798 Australian and British adults (aged 18 to 54) who were asked about their experiences of digital harassment and abuse:

...the findings highlight the importance of actively promoting safe and inclusive online spaces. Although the law is a part of the solution (through hate speech and anti-discrimination legislation, for example), the policies and practices of social media and online platform providers are also important for challenging and preventing such behaviour, using tools such as community standards and reporting functions. Other sectors of society, such as police, as well as educational and governmental institutions, likewise play a crucial role in challenging cultures and practices that tolerate digital harassment and abuse. (p. 218)

This quote highlights the need to tackle the negative impacts of media and technology through multiple levels of education and intervention. These could include providers of social media companies monitoring and blocking inappropriate and harmful 
material, policy and guidelines on ethical use of technologies and community standards, and campaigns to raise public awareness on ethical digital citizenship (Henry \& Powell, 2016).

\section{SECTION SUMMARY}

In summary, a significant effort is needed to address dating violence and the underlying influences and impacts. Dating violence is a complex issue and requires a comprehensive approach to its elimination, applying the public health approach, leveraging off evidence-based programmes, alongside culturally specific application, and rigorous evaluation (Beres, 2017). Although there has been a large increase in attention on dating violence since Makepeace's (1981) seminal study, there are still a number of limitations across this body of work that impact on our ability to adequately understand and prevent this type of violence for young people (Lewis \& Fremouw, 2001).

What the findings of this study do support is the utility of Johnson's typology in demonstrating that there are different types of violence experienced by young adults in dating relationships, requiring multiple models and approaches in the prevention and intervention of dating violence. In other words, a diverse approach is required to effectively prevent dating violence (Gulliver \& Fanslow, 2016).

Diverse approaches need to acknowledge and address different types of violence that have their own patterns, drivers and outcomes across the ecological system, particularly acknowledging that dating violence in New Zealand is strongly influenced by the current social context, a context that brings its own risks and opportunities within the normalised context of hooking-up, engagement in the nighttime economy, and the proliferation of media and technology.

No 'one-size-fits-all' model works for all trauma- and violence-informed services. Individual services need a structure that suits the community they work in and is responsive to complex and chronic trauma of individuals and communities. (Family Violence Death Review Committee, 2020, p. 17)

Noel et al. (2016) conclude from their study of dating that there are inherent risks in the now typical dating process, particularly for women. However, rather than depicting this now 'normalised' way of meeting/hooking-up as inherently harmful, we 
can focus on creating conditions for appropriate, meaningful and safe engagement within this changed environment (Konstam, 2019).

\section{CONCLUSION AND FINAL REFLECTIONS}

In conclusion, this research has identified two overarching findings. First, there is a large volume of dating violence experienced in the social context of New Zealand, which has parallels with trends within a broader youth culture. Second, one way to understand and intervene in such violence is to apply an approach, such as Johnson's typology, to unpick the different aetiologies of violence.

This research provides positive support for Johnson's (2008) typology in showing that there are different types of violence in intimate relationships, in this case in dating relationships. What Johnson's approach emphasises is the need to understand, validate and find solutions to dating violence that occur in a range of different situations, where there are different contexts, drivers, and impacts. While my analysis suggests that Johnson's typology has merit and provides a platform for a required and ongoing conversation on the different types and experiences of violence that occur, there are also some risks in applying such a typology, as discussed in Chapter Three. Whether it is through further development of Johnson's typology or of other typological models, this study provides evidence that the complexity of dating violence is better captured through a framework that does not view all dating violence as a unitary phenomenon. Acknowledging and understanding more about the different types of violence in dating relationships allows for differential responses and approaches to be developed that address the varied experiences of dating violence.

Finally, this thesis has demonstrated that a large majority of young adults experience some form of violence in their dating relationships, from more minor episodes to relationships characterised by severe and terrifying violence. Further, we need to view dating violence as a multi-faceted, complex and dynamic interaction between intimate partners. This requires a sophisticated conceptual framework, such as Johnson's typology, that captures the heterogeneous and complex nature of violence in dating relationships.

This study holds particular significance as one of the few New Zealand-based analyses of violence occurring in the context of contemporary New Zealand dating 
relationships by young adults. The findings provide strong support for the need to focus on a range of interventions and programmes delivered to young adults who are experiencing different types of violence. The findings also underscore the importance of critically analysing media messages, challenging traditional gender norms, and fighting against attitudes and beliefs that support and validate the use of violence in dating relationships.

The young adults in this study described how they navigated through a somewhat contradictory landscape between more relaxed attitudes to relationships, casual sex and drinking, contrasted with the need to adhere to more traditional expectations about gendered roles in relationships. Since these interviews took place, the social context that young adults are engaging is has continued to change at a rapid pace particularly around the proliferation of social media, popular culture and technology. This was reflected by the recent murder of Grace Millane - detailed in Chapter Eight. Grace's murder shone a spotlight on the fast-changing global youth context, with the media coverage showcasing changes in the ways that young adults engage in dating and sexual interactions. Grace's case, and the stories of those captured in this thesis, show how young people seeking to date and hook-up are increasingly engaging with technology, and how this can shape their social context. The proliferation of technology has redefined relationships - both how they are established, including through the use of dating apps, as well as how young adults engage through the dating process.

This thesis also highlights, through the application and utility of Johnson's (2008) typology, that we must acknowledge the diverse range of behaviours and practices that young adults use to maintain power and control over their partner. This has been well-recognised elsewhere, with Flood (2019) observing:

There is now considerable evidence that there are different types of domestic violence, with differing causes, dynamics, and impacts. Michael Johnson's work provides the most developed instance of this recognition. (p. 24)

Therefore, to successfully address the complexities of dating violence within this current and changing social context we need to respond across individual, relationship, community, institutional, and societal levels with a spectrum of 
evidence-based prevention (Flood, 2019). We need to normalise conversations and education around good positive relationships, dismantle the traditional romantic narratives, educate young adults to critically assess hyper-sexualised media and pornographic material, emphasise building self-esteem and learning the skills of positive communication, educate young people on expectations around ethical sexual relationships, and express positive sexual identity (Noel et al., 2016; Jackson \& Vares, 2015b; Beres \& Farvid, 2010; Hird \& Jackson, 2001; Lewis \& Fremouw, 2001; Towns \& Adams, 2000; Jackson, 1998a). The eradication of the pervasive social problem of dating violence and other forms of IPV is unlikely while structural gender inequalities persist. What is within our scope, however, is the potential to address the widespread cultural norms, constructions of gender, and patterns of control that contribute to dating violence, and to implement prevention initiatives aimed at reducing their occurrence (Flood, 2019; Beres, 2017; Towns \& Scott, 2013). 


\section{REFERENCES}

Ackerman, J. (2018). Assessing Conflict Tactics Scale Validity by Examining Intimate Partner Violence Over-reporting. Psychology of Violence, 8 (2), 207217.

Adams, H., \& Williams, L. (2014). It's Not Just You Two': A grounded theory of peer-influenced jealousy as a pathway to dating violence among acculturating Mexican American adolescents. Psychology of Violence, 4 (3), 294-308.

Adams, P., Towns, A., \& Gavey, N. (1995). Dominance and Entitlement: The rhetoric men use to discuss their violence towards women. Discourse and Society, 60, 387-406.

Allen, L. (2004). "Getting Off" and "Going Out": Young people's conceptions of (hetero)sexual relationships. Culture, Health and Sexuality, 6 (6), 463-481.

Anderson, K. (2008). Is Partner Violence Worse in the Context of Control? Journal of Marriage and Family, 70 (5), 1157-1168.

Anderson, K. (2009). Review: A Typology of Domestic Violence: Intimate terrorism, violent resistance and situational couple violence by Michael Johnson. Contemporary Sociology: A Journal of Reviews, 38 (6), 532-533.

Appleton-Dyer, S., Soupen, A., \& Edirisuriya, N. (2017). Report on the 2016 Mates and Dates Survey: Report for the Violence Prevention portfolio at ACC. Auckland: Synergia.

Archer, J. (2000). Sex Differences in Aggression Between Heterosexual Partners: A meta-analytic review. Psychological Review, 126 (5), 651-680.

Babcock, J., Snead, A., Bennett, V., \& Armenti, N. (2019). Distinguishing Subtypes of Mutual Violence in the Context of Self-Defense: Classifying types of partner violent couples using a modified Conflict Tactics Scale. Journal of Family Violence, 34 (7), 687-69.

Baker, C., \& Carreno, P. (2016). Understanding the Role of Technology in Adolescent Dating and Dating Violence. Journal of Child and Family Studies, $25,308-320$.

Baker, G. (2013). Effectively Involving Men in Preventing Violence Against Women. University of Auckland. New Zealand Family Violence Clearinghouse.

Baker, M., \& Elizabeth, V. (2013). "Did you just ask me to marry you?': The gendered nature of heterosexual relationship progression. Women's Studies Journal, 27 (2), 32-43.

Baker, M., \& Elizabeth, V. (2012). Negotiating 'Marriage': Comparing same-sex and different sex cohabiting couples. New Zealand Sociology, 27 (2), 10-28. 
Banyard, V., Potter, S., Cares, A., Williams, L., Moynihan, M., \& Stapleton, J. (2018). Multiple Sexual Violence Prevention Tools: Doses and boosters. Journal of Aggression, Conflict and Peace Research, 10 (2), 145-155.

Barnett, M., Hale, T., \& Sligar, K. (2017). Masculinity, Femininity, Sexual Dysfunctional Beliefs, and Rape Myth Acceptance Among Heterosexual College Men and Women. Sexuality and Culture, 21, 741-753.

Barnett, O., Miller-Perrin, C., \& Perrin, R. (2005). Family Violence Across the Lifespan: An introduction . London: Sage Publication.

Bates, E. (2016). Current Controversies Within Intimate Partner Violence:

Overlooking bidirectional violence. Journal of Family Violence, 31, 937-940.

Bates, E., Graham-Kevan, N., \& Archer, J. (2014). Testing Predictions from the Male Control Theory of Men's Partner Violence. Aggressive Behviour, 40 (1), 4255.

Bay-Cheng, L., \& Goodkind, S. (2016). Sex and the Single (Neoliberal) Girl: Perspectives on being single among socioeconomically diverse young women. Sex Roles, 74 (5), 181-194.

Becker, S., \& Tinkler, J. (2015). "Me Getting Plastered and Her Provoking My Eyes": Young people's attribution of blame for sexual aggression in public drinking spaces. Feminist Criminology, 10 (3).

Belknap, J., \& Melton, H. (2005). Are Heterosexual Men Also Victims of Intimate Partner Abuse? From http://www.vawnet.org

Belsky, J. (1980). Child maltreatment: An ecological integration. American Psychologist, 35 (4), 320-335.

Beres, M. (2007). "Spontaneous" Sexual Consent: An analysis of sexual consent literature. Feminism and Psychology, 17 (1), 93-108.

Beres, M. (2020). Perspectives of Rape-Prevention Educators on the Role of Consent in Sexual Violence Prevention. Sex Education, 20 (2), 227-238.

Beres, M. (2014a). Points of Convergence: Introducing Sexual Scripting Theory to discourse approaches to the study of sexuality. Sexuality and Culture, 18, 7688 .

Beres, M. (2017). Preventing Adolescent Relationship Abuse and Promoting Healthy Relationships. Auckland, New Zealand: New Zealand Family Violence Clearinghouse.

Beres, M. (2014b). Rethinking the Concept of Consent for Anti-Sexual Violence Activism and Education. Feminism and Psychology, 24 (3), 373-389.

Beres, M. (2010). Sexual Miscommunication? Untangling assumptions about sexual communication between casual sex partners. Culture, Health and Sexuality, 12 (1), 1-14. 
Beres, M., \& Farvid, P. (2010). Girls, Young Women and their sexualities. Sexualities, 13 (3), 377-393.

Beres, M., \& MacDonald, J. (2015). Talking About Sexual Consent, Heterosexual Women and BDSM. Australian Feminist Studies, 30 (86), 418-432.

Beres, M., Senn, C., \& McCaw, J. (2014). Navigating Ambivalence: How heterosexual young adults make sense of desire differences. Journal of Sex Research, 51 (7), 765-776.

Beres, M., Terry, G., Senn, C., \& Ross, L. (2019). Accounting for Men's Refusal of Heterosex: A story-completion study with young adults. The Journal of Sex Research, 56 (1), 157-136.

Beres, M., Treharne, G., \& Stojanov, Z. (2019). A Whole of Campus Approach to Sexual Violence: The University of Otago model. Journal of Higher Education Policy and Management, 41 (6), 646-662.

Beres, M., Treharne, G., Stewart, K., Flett, J., Rahman, M., \& Lillis, D. (2019c). A Mixed-Methods Pilot Study of the EAAA Rape Resistance Programme for Female Undergraduate Students in Aotearoa/New Zealand. Women's Studies Journal, 33 (1/2), 8-24.

Berger, R. (2015). Now I See It, Now I Don't: Researcher's position and reflexivity in qualitative research. Qualitative Research, 15 (2), 219-234.

Berkowitz, A. (2004). Working with Men to Prevent Violence Against Women: An overview (part one). National Resource Center on Domestic Violence, 9 (2), $1-7$.

Bernard, M., \& Bernard, J. (1983). Violent Intimacy: The family as a model for love relationships. Family Relations, 32, 283-286.

Black, M., Basile, K., Breiding, M., \& Smith, S. (2010). The National Intimate Partner and Sexual Violence Survey: 2010 Summary Report.

Black, M., Basile, K., Breiding, M., Smith, S., Walters, M., Merrick, M., et al. (2011). The National Intimate Partner and Sexual Violence Survey (NISVS): 2010 Summary Report. Centers for Disease Control and Prevention. Atlanta, GA: National Center for Injury Prevention and Control.

Bogaerts, S., van der Veen, H., \& van der Knaap, L. (2011). Aspects of Intimate Terrorism: A test of Johnson's Typology in a Dutch Online Panel. International Perspectives in Victimology, 5 (1), 13-21.

Bonnie, R. J., Stroud, C., \& Breiner, H. (2015). Investing in the Health and WellBeing of Young Adults. Committee on Improving the Health, Safety, and WellBeing of Young Adults; Board on Children, Youth and Families. Washington: National Academies Press.

Booker, J. (2009). Retrieved March 2018 from New Zealand Herald: https://www.nzherald.co.nz/nz/news/article.cfm?c_id=1\&objectid=10580426 
Borsky, A., McDonnell, K., Rimal, R., \& Turner, M. (2016). Assessing Bystander Behavior Intentions Towards Friends to Prevent Dating Violence: Development of the Bystander Behavior Intentions-Friends Scale through concept mapping and exploratory factor analysis. Violence and Victims, 31 (2), 215-234.

Braithwaite, S., Coulson, G., Keddington, K., \& Fincham, F. (2015). The Influence of Pornography on Sexual Scripts and Hooking Up Among Emerging Adults in College. Archives of Sexual Behavior, 44, 111-123.

Brecklin, L., \& Firde, D. (2001). A Meta-Analysis of Rape Education Programs. Violence and Victims, 16 (3), 303-321.

Breger, M. (2017). Reforming by re-norming: How the legal system has the potential to change toxic culture of domestic violence. Journal of Legislation, 44 (2), 170-200.

Bronfenbrenner, U. (1979). The Ecology of Human Development. Cambridge: Harvard University Press.

Brown, J., James, K., \& Taylor, A. (2010). Caught in the Rejection-Abuse Cycle: Are we really treating perpetrators of domestic violence effectively? Journal of Family Therapy, 32, 280-307.

Brownridge, D. (2006). Intergenerational Transmission and Dating Violence Victimization: Evidence from a Sample of Female University Students in Manitoba . Canadian Journal of Community Mental Health, 25 (1), 75-93.

Bruton, C., \& Tyson, D. (2018). Leaving violent men: A study of women's experiences of separation in Victoria, Australia. Australian and New Zealand Journal of Criminology, 51 (3), 339-354.

Burck, C. (2005). Comparing Qualitative Research Methodologies for Systemic Research: The use of grounded theory, discourse analysis and narrative analysis. Journal of Family Therapy, 27, 237-262.

Butler, J. (1994). Gender as Performance. Radical Philosophy, 67, 32-39.

Butler, J. (1988). Performative Acts and Gender Constitution: An essay in phenomenology and feminist theory. Theatre Journal, 40 (4), 519-531.

Cale, J., Tzoumakis, S., Leclerc, B., \& Breckenridge, J. (2016). Patterns of Intimate Partner Violence Victimization Among Australia and New Zealand University Students: An initial examination of child maltreatment and self-reported depressive symptoms across profiles. Australian and New Zealand Journal of Criminology, 1-20.

Capaldi, D., \& Owen, L. (2001). Physical Aggression in a Community Sample of AtRisk Young Couples: Gender comparisons for high frequency, injury, and fear. Journal of Family Violence, 15 (3), 425-440. 
Capaldi, D., Kim, H., \& Shortt, J. (2007). Observed Initiation and Reciprocity of Physical Aggression in Young, At-Risk Couples. Journal of Family Violence, 22 (2), 101-111.

Carmody, M., \& Ovenden, G. (2013). Putting Ethical Sex Into Practice: Sexual negotiation, gender and citizenship in the lives of young women and men. Journal of Youth Studies, 16 (6), 792-807.

Chan, K. (2012). The Role of Chinese Face in the Perpetration of Dating Partner Violence. Journal of Interpersonal Violence, 27, 793-811.

Charmaz, K. (2006). Constructing Grounded Theory: A practical guide through qualitative analysis. Thousand Oaks: Sage Publications.

Charmaz, K., \& Belgrave, L. (2019). Thinking About Data With Grounded Theory. Qualitative Enquiry, 25 (8), 743-753.

Chiodo, D., Crooks, C., Wolfe, D., McIsaac, C., Hughes, R., \& Jaffe, P. (2012). Longitudinal Prediction and Concurrent Functioning of Adolescent Girls Demonstrating Various Profiles of Dating Violence and Victimization. Prevention Science, 13, 350-359.

Chung, D. (2005). Violence, Control, Romance, and Gender Equality: Young women and heterosexual relationships. Women's Studies International Forum, 28 (6), 445-455.

Classification Office. (2020). Growing Up With Porn: Insights from young New Zealanders. Wellington, NZ: Classification Office.

Coker, A., Bush, H., Cook-Craig, P., Degue, S., Clear, E., Brancato, C., et al. (2017). RCT Testing Bystander Effectiveness to Reduce Violence. American Journal of Preventative Medicine, 52 (5), 566-578.

Coker, A., Follingstad, D., Bush, H., \& Fisher, B. (2016). Are Interpersonal Violence Rates Higher Among Young Women in College Compared With Those Never Attending College? Journal of Interpersonal Violence, 31 (8), 1413.

Conger, R., Cui, M., Bryant, C., \& Elder, G. (2001). Competence in Early Adult Romantic Relationships: A developmental perspective on family influences. Prevention and Treatment, 1 (4).

Connell, R. (1987). Gender and Power: Society, the person and sexual politics. Sydney: Allen \& Unwin.

Connell, R. (1995). Masculinities. Cambridge, UK: Polity Press.

Connell, R. (2002). Studying Men and Masculinity. Resources for Feminist Research, $29(1 / 2), 43-56$.

Connell, R., \& Messerschmidt, J. (2005). Hegemonic Masculinity: Rethinking the concept. Gender and Society, 19 (6), 829-859.

Conroy, N., Krishnakumar, A., \& Leone, J. (2015). Re-examining Issues of Conceptualization and Willing Consent: The hidden role of coercion in 
experiences of sexual acquiescence. Journal of Interpersonal Violence, 30 (11), 1828-1846.

Currie, D. (1998). Violent Men or Violent Women? Whose definition counts. In R. Bergen, Issues in Intimate Violence (pp. 97-111). Thousand Oaks, CA: Sage Publications.

Daigle, L., Johnston, T., Azimi, A., \& Felix, S. (2019). Violent and Sexual Victimization Among American and Canadian College Students: Who Is More at Risk and Are the Risk Factors Invariant? Journal of School Violence, 18 (2), 226-240.

Danielsson, I., Blom, H., Nilses, C., Heimer, G., \& Hogberg, U. (2009). Gendered Patterns of High Violence Exposure Among Swedish Youth. Acta Obstetricia et Gynecologica Scandinavica, 88 (5), 528-535.

Dank, M., Lachman, P., Zweig, J., \& Yahner, J. (2014). Dating Violence Experiences of Lesbian, Gay, Bisexual, and Transgender Youth. Journal of Youth and Adolescence, 43, 846-857.

Danube, C., Vescio, T., \& Davis, K. (2014). Male Role Norm Endorsement and Sexism Predict Heterosexual College Men's Attitudes Toward Casual Sex, Intoxicated Sexual Contact, and Casual Sex. Sex Roles, 71 (5), 219-232.

Dardis, C., Dixon, K., Edwards, K., \& Turchik, J. (2015). An Examination of the Risk Factors Related to Dating Violence Perpetration Among Young Men and Women and Associated Theoretical Explanations: A review of the literature. Trauma, Violence and Abuse, 16 (2), 136-152.

Day, A., \& Bowen, E. (2015). Offending Competency and Coercive Control in Intimate Partner Violence. Aggression and Violent Behavior, 20, 62-71.

Day, K., Gough, B., \& McFadden, M. (2004). "Warning! Alcohol Can Seriously Damage Your Feminie Health": A discourse analysis of recent British newspaper coverage of women and drinking. Feminist Media Studies, 4 (2).

DeKerseredy, W., \& Schwartz, M. (1998). Woman Abuse on Campus: Results from the Canadian National Survey. Thousand Oaks, CA: Sage Publications.

Dobash, R., \& Dobash, R. (2004). Women's Violence To Men in Intimate Relationships: Working on a puzzle. British Journal of Criminology, 11, 324349.

Dobash, R., Dobash, R., Wilson, M., \& Daly, M. (1992). The Myth of Sexual Symmetry in Marital Violence. Social Problems, 39, 71-91.

Dobbs, T., \& Eruera, M. (2014). Kaupapa Māori Wellbeing Framework: The basis for whānau violence prevention. New Zealand Family Violence Clearinghouse.

Douglas, E., \& Straus, M. (2006). Assault and Injury of Dating Partners by University Students in 19 Countries and its Relation to Corporal Punishment Experienced as a Child. European Journal of Criminology, 3 (293-318). 
Dutton, D., \& Nicholls, T. (2005). The Gender Paradigm in Domestic Violence Research and Theory: Part 1 - the conflict of theory and data. Aggression and Violent Behavior, 68, 701-717.

Dutton, M., \& Goodman, L. (2005). Coercion in Intimate Partner Violence: Toward a new conception. Sex Roles, 52, 743-756.

Dutton, M., Kaltman, S., Goodman, L., Weinfurt, K., \& Vankos, N. (2005). Patterns of Intimate Partner Violence: Correlates and outcomes. Violence and Victims, 20 (5), 483-497.

Eagly, A. (1987). Sex Differences in Social Behavior: A Social-Role Interpretation. Taylor \& Francis Group.

Eaton, A., \& Rose, S. (2011). Has Dating Become More Egalitarian? A 35 year review using sex roles. Sex Roles, 64, 843-862.

Eggleston, E. (2000). New Zealand Youth Gangs: Key findings and recommendations from an urban ethnography. Social Policy Journal of New Zealand .

Ellsberg, M., Arango, D., Morton, M., Gennari, F., Kiplesund, S., Contreras, M., et al. (2015). Prevention of Violence Against Women and Girls: What does the evidence say? The Lancet, 385 (9977), 1555-1566.

Enander, V. (2011). Violent Women? The challenge of women's violence in intimate heterosexual relationships to feminist analyses of partner violence. Journal of Feminist and Gender Research, 19 (2), 105-123.

Eruera, M., \& Dobbs, T. (2010). Taitamariki Māori Kōrero About Intimate Partner Relationships. Whangarei: Amokura Family Violence Prevention Consortium.

Espinoza, R., \& Warner, D. (2016). Where Do We Go From Here?: Examining intimate partner violence by bringing male victims, female perpetrators, and psychological sciences into the fold. Journal of Family Violence, 31, 959-966.

Family Violence Death Review Committee. (2017). Family Violence Death Review Committee Fifth Report Data: January 2009 to December 2015.

Family Violence Death Review Committee. (2020). Sixth report: Te Pürongo tuaono: Men who use violence, Ngā tāne ka whakamahi i te whakarekereke. Wellington: Health Quality and Safety Commission.

Fanslow, J. (2005). Beyond Zero Tolerance: Key issues and future directions for family violence work in New Zealand. Families Commission.

Fanslow, J. (2017). Intimate Partner Violence and Women's Reproductive Health. Obstetrics, Gynaecology and Reproductive Health, 27 (5), 148-157.

Fanslow, J., \& Robinson, E. (2004). Violence Against Women in New Zealand: Prevalence and health consequences. The New Zealand Medical Journal, 117 (1206). 
Farvid, P., \& Braun, V. (2014). The "Sassy Woman" and the "Performing Man": Heterosexual casual sex advice and the (re)constitution of gendered subjectivities. Feminist Media Studies, 14 (1), 118-134.

Fileborn, B. (2017). 'Staff Can't be the Ones that Play Judge and Jury': Young adults' suggestions for preventing unwanted sexual attention in pubs and clubs. Australian and New Zealand Journal of Criminology, 50 (2), 213-233.

Fisher, B., Daigle, L., \& Cullen, F. (2010). Unsafe in the Ivory Tower: The sexual victimization of college women. Thousand Oaks: Sage Publications, Inc.

Flood, M. (2019). Engaging Men and Boys in Violence Prevention. Pelgrave Macmillan.

Flood, M. (2006). The Debate Over Men's Versus Women's Family Violence. Family Violence Conference (pp. 1-12). Adelaide: Australian Institute of Judicial Administration.

Flood, M., \& Pease, B. (2009). Factors Influencing Attitudes to Violence Against Women. Trauma, Violence and Abuse, 10 (2), 125-142.

Fonow, M., \& Cook, J. (2005). Feminist Methodology: New applications in the academy and public policy. Signs, 30, 2211-2236.

Foshee, V. (1996). Gender Differences in Adolescent Dating Abuse Prevalence, Types and Injuries. Health Education Research, 11, 275-286.

Foshee, V., Bauman, A., Ennett, K., Linder, S., Benefield, T., \& Suchindran, C. (2004). Assessing the Long-Term Effects of the Safe Dates Program and a Booster in Preventing and Reducing Adolescent Dating Violence Victimization and Perpetration. American Journal of Public Health, 94 (4), 619-624.

Foshee, V., Bauman, K., Linder, F., Rice, J., \& Wilcher, R. (2007). Typologies of Adolescent Dating Violence: Identifying typologies of adolescent dating violence perpetration. Journal of Interpersonal Violence, 22, 498-519.

Freedner, N., Freed, L., Yang, W., \& Austin, S. (2002). Dating Violence Among Gay, Lesbian, and Bisexual Adolescents: Results from a community survey. Journal of Adolescent Health, 31, 469-474.

Frieze, I. (2005). Female Violence Against Intimate Partners: An introduction. Psychology of Women Quarterly, 29, 229-237.

Frieze, I. (1983). Investigating the Causes and Consequences of Marital Rape. Signs, $8(3), 532-553$.

Frieze, I., \& Browne, A. (1989). Violence in Marriage. In 1. Ohlin, \& M. Tonry, Family Violence (pp. 163-218). Chicago: University of Chicago Press.

Fry, M. (2011). Seeking the Pleasure Zone: Understanding young adult's intoxication culture. Australasian Marketing Journal, 19, 65-70. 
Frye, V., Manganello, J., Campbell, J., Walton-Moss, B., \& Wilt, S. (2006). The Distribution and Factors Associated With Intimate Terrorism and Situational Couple Violence Among a Population-Based Sample of Urban Women in the United States. Journal of Interpersonal Violence, 21 (10), 1286-1313.

Fuentes, M., \& Gunder Frank, A. (1989). Ten Theses on Social Movements. World Development, 17 (2), 179-191.

Gallaty, K., \& Zimmer-Gembeck, M. (2008). The Daily Social and Emotional Worlds of Adolescents Who Are Psychologically Maltreated by Their Romantic Partners. Journal of Youth and Adolescence, 37 (3), 310-323.

Garcia, T., Litt, D., Davis, K., Norris, J., Kaysen, D., \& Lewis, M. (2019). Growing Up, Hooking Up, and Drinking: A review of uncommitted sexual behavior and its association with alcohol use and related consequences among adolescents and young adults in the United States. Frontiers in Psychology, 10, 1872.

Gavey, N. (2019). Just Sex? The Cultural Scaffolding of Rape (2nd edition ed.). London and New York: Routledge.

Gavey, N. (1991). Sexual Victimization Prevalence Among New Zealand University Students. Journal of Consulting and Clinical Psychology, 59 (13), 464.

Gibbs, A., Dunkle, K., Ramsoomar, L., Willan, S., Shai, N., Chatterji, S., et al. (2020). New Learnings on Drivers of Men's Physical and/or Sexual Violence Against Their Female Partners, and Women's Experiences of this, and the Implications for Prevention Interventions. Global Health Action, 13 (1), 1-12.

Gillum, T., \& DiFulvio, G. (2012). "There's So Much at Stake": Sexual minority youth discuss dating violence. Violence Against Women, 18 (7), 725-745.

Glass, N., Laughon, K., Campbell, J., Wolf Chair, A., Block, C., Hanson, G., et al. (2008). Non-Fatal Strangulation is an Important Risk Factor for Homicide of Women. Journal of Emergency Medicine, 35 (3), 329-335.

Graham-Kevan, N., \& Archer, J. (2008). Does Controlling Behavior Predict Physical Aggression and Violence to Partners? Journal of Family Violence, 23 (7), 539548.

Graham-Kevan, N., \& Archer, J. (2003). Intimate Terrorism and Common Couple Violence: A test of Johnson's predictions in four British samples. Journal of Interpersonal Violence, 18 (11), 1247-1270.

Graham-Kevan, N., \& Archer, J. (2004). Using Johnson's Domestic Violence Typology to Classify Men and Women in a Non-selected Sample. University of Central Langcashire, Department of Psychology. Preston.

Griffin, C., Szmigin, I., Bengry-Howell, A., Hackley, C., \& Mistral, W. (2013). Inhabiting the Contradictions: Hypersexual femininity and the culture of intoxication among young women in the UK. Feminism and Psychology, 23 (2), 184-206. 
Gulliver, P., \& Fanslow, J. (2015). The Johnson Typologies of Intimate Partner Violence: An investigation of their representation in a general population of New Zealand women. Journal of Child Custody, 12 (1), 25-46.

Gulliver, P., \& Fanslow, J. (2016). Understanding Research on Risk and Protective Factors for Intimate Partner Violence. New Zealand Family Violence Clearinghouse.

Hamberger, L. K., \& Guse, C. E. (2002). Men's and Women's Use of Intimate Partner Violence in Clinical Samples. Violence Against Women, 8, 1301-1331.

Hamberger, L. (2005). Men's and Women's Use of Intimate Partner Violence in Clinical Studies: Toward a gender-sensitive analysis. Violence and Victims, 20 (3), 131-151.

Hamilton, L., \& Armstrong, E. (2010). Gendered Sexuality in Young Adulthood. Gender and Society, 23 (5), 589-616.

Hammock, G., Richardson, D., Lamm, K., Taylor, E., \& Verlaque, L. (2017). The Effect of Gender of Perpetrator and Victim on Perceptions of Psychological and Physical Intimate Partner Aggression. Journal of Family Violence, 32, 357-365.

Harned, M. (2002). A Multivariate Analysis of Risk Markers for Dating Violence Victimization. Journal of Interpersonal Violence, 17 (11).

Harned, M. (2001). Abused Women or Abused Men? An examination of the context and outcomes of dating violence. Violence and Victims, 16 (3), 269-285.

Harris, A., Honey, N., Webster, K., Diemer, K., \& Politoff, V. (2015). Young Australians' Attitudes to Violence Against Women: Findings from the 2013 National Community Attitudes towards Violence Against Women Survey for respondents 16-24 years. Melbourne, Australia: Victorian Health Promotion Foundation.

Hautala, D., Sittner Hartshorn, K., Armenta, B., \& Whitbeck, L. (2017). Prevalence and Correlates of Physical Dating Violence Among North American Indigenous Adolescents. Youth and Society, 49 (3), 295-317.

Heise, L. (1998). Violence Against Women: An Integrated Ecological Framework. Violence Against Women, 4 (3), 262-290.

Heith, C., \& Miller, J. (2015). Criminology's Theoretical Incarceration: Qualitative Methods as Liberator. In The Routledge Handbook of Qualitative Criminology (pp. 38-47). Routledge.

Hendrickson, M. (2007). Reaching Out, Hooking Up: Lavender netlife in a New Zealand Study. Sexuality Research and Social Policy, 4 (2), 38-49.

Henry, N., \& Powell, A. (2016). Sexual Violence in the Digital Age: The scope and limits of criminal law. Social and Legal Studies, 25 (4), 397-418. 
Hines, D., \& Douglas, E. (2010). Intimate Terrorism by Women Towards Men: Does it exist? Journal of Aggression, Conflict and Peace Research, 2 (3), 36-56.

Hines, D., Brown, J., \& Dunning, E. (2007). Characteristics of Callers to the Domestic Abuse Helpline for Men. Journal of Family Violence, 22 (2), 63-72.

Hird, M., \& Jackson, S. (2001). Where 'angels' and 'wusses' Fear to Tread: Sexual Coercion in Adolescent Dating Relationships. Journal of Sociology, 37 (1), 27.

Hoffman, R. (2018). Dating and Mating in a Techno-Driven World: Understanding how technology is helping and hurting relationships. Santa Barbara: Praegar.

Holtzworth-Munroe, A. (2000). A Typology of Men Who Are Violent Toward Their Female Partners: Making sense of the heterogeneity in husband violence. Current Directions in Psychological Science, 9 (4), 140-143.

Holtzworth-Munroe, A. (2005). Female Perpetration of Physical Aggression Against an Intimate Partner: A controversial new topic of study. Violence and Victims, 20 (2), 251-259.

Holtzworth-Munroe, A., \& Stuart, G. (1994). Typologies of Male Batterers: Three subtypes and the differences among them. Psychological Bulletin, 116, 476497.

Humphreys, T., \& Herold, E. (2007). Sexual Consent in Heterosexual Relationships: Development of a new measure. Sex Roles, 57 (3-4), 305-315.

Hunnicutt, G. (2009). Varieties of Patriarchy and Violence Against Women: Resurrecting 'patriarchy' as a theoretical tool. Violence Against Women, 15 (5), 553-573.

Hunter, E. (2009). Courtship. In J. O'Brien, Encyclopaedia of Gender and Society (pp. 169-171). Thousand Oaks, CA: Sage Publications.

Hutton, F. (2012). Harm Reduction, Students and Pleasure: An examination of student responses to a binge drinking campaign. International Journal of Drug Policy, $23,229-235$.

Hutton, F., Griffin, C., Lyons, A., Niland, P., \& McCreanor, T. (2016). "Tragic Girls" and "Crack Whores": Alcohol, femininity and Facebook. Feminism and Psychology, 26 (1), 73-93.

Hutton, F., Wright, S., \& Saunders, E. (2013). Cultures of Intoxication: Young women, alcohol, and harm reduction. Contemporary Drug Problems, 40, 451480 .

Jackson, S. (2002). Abuse in Dating Relationships: Young people's accounts of disclosure, non-disclosure, help-seeking and prevention education. New Zealand Journal of Psychology, 31 (2), 79-86.

Jackson, S. (1998b). Family Violence and Teenage Dating Trouble: Is there a connection. Social Work Now, 9, 31-36. 
Jackson, S. (2001). Happily Never After: Young Women's Stories of Abuse in Heterosexual Love Relationships. Feminism and Psychology, 11 (3), 305-321.

Jackson, S. (1999). Issues in the Dating Violence Research: A review of the literature. Aggression and Behavior, 4 (2), 233-247.

Jackson, S. (1998a). Teenage Dating Violence. A thesis submitted for the degree of Doctor of Philosophy in Psychology. University of Auckland.

Jackson, S., \& Vares, T. (2015a). 'Perfect Skin', 'Pretty Skinny': Girl's embodied identities and post-feminist popular culture. Journal of Gender Studies, 24 (3), 347-360.

Jackson, S., \& Vares, T. (2015b). 'Too Many Bad Role Models for us Girls': Girls, female pop celebrities and 'sexualisation'. Sexualities, 18 (4), 480-498.

James, B. (2010). Under the Influence: Reshaping New Zealand's drinking culture. The Salvation Army Social Policy and Parliamentary Unit.

Jasinski, J., Blumenstein, L., \& Morgan, R. (2014). Testing Johnson's Typology: Is there gender symmetry in Intimate Terrorism? Violence and Victims, 29 (1), 73-88.

Javaid, A. (2018). Male Rape, Masculinities, and Sexualities. International Journal of Law, Crime and Justice, 52, 199-210.

Jewkes, R., Flood, M., \& Lang, J. (2015). From Work With Men and Boys to Changes of Social Norms and Reduction in Inequalities in Gender Relations: A conceptual shift in prevention of violence against women and girls. The Lancet, 385.9977, 1580-1589.

Johnson, M. (2017). A Personal Social History of a Typology of Intimate Partner Violence. Journal of Family Theory and Review, 9, 150-164.

Johnson, M. (2008). A Typology of Domestic Violence: Intimate Terrorism, Violent Resistance, Situational Couple Violence. Boston: Northeastern University Press.

Johnson, M. (2009). Differentiating Among Types of Domestic Violence: Implications for healthy marriages. In E. Peters, \& D. Kamp, Marriage and Family: Perspectives and Complexities (pp. 281-297). New York: Columbia University Press.

Johnson, M. (2014). Distinguishing Among Types of Domestic Violence. In M. Rinfret-Raynor, E. Lesieux, M. Cousineau, S. Gauthier, \& E. Harper (Eds.), Violences Envers les Femmes: Realities Complexes et Nouveaux Enjeux dans un Monde en Transformation. Quebec: Presses Universitaires de l'Universite du Quebec.

Johnson, M. (1995). Patriarchal Terrorism and Common Couple Violence: Two forms of violence against women in U.S. families. Journal of Marriage and the Family, 57, 283-294. 
Johnson, M., \& Ferraro, K. (2000). Research on Domestic Violence in the 1990s: Making distinctions. Journal of Marriage and the Family, 62 (4), 948-963.

Johnson, M., \& Leone, J. (2005). The Differential Effects of Intimate Terrorism and Situational Couple Violence: Findings from the National Violence Against Women Survey. Journal of Family Issues, 26, 322-349.

Johnson, M., Leone, J., \& Xu, Y. (2008). Gender, Intimate Terrorism and Situational Couple Violence in Survey Data. National Council on Family Relations annual meeting. Little Rock, Arkansas.

Johnson, M., Leone, J., \& Xu, Y. (2014). Intimate Terrorism and Situational Couple Violence in General Surveys: Ex-spouses required. Violence Against Women, 20 (2), 186-207.

Jordan, J., \& Mossman, E. (2019). Get Out of My Home and Don't Come Back!: Empowering women through self-defense. Violence Against Women, 25 (3), 313-336.

Katz, J., Kuffel, S., \& Brown, F. (2006). Leaving A Sexually Coercive Dating Partner: A prospective application of the investment model. Psychology of Women Quarterly, 30, 267-275.

Katz, J., Washington Kuffel, S., \& Coblentz, A. (2002). Are There Gender Differences in Sustaining Dating Violence? An examination of frequency, severity, and relationship satisfaction. Journal of Family Violence, 17 (3), 247-271.

Kaura, S., \& Allen, C. (2004). Dissatisfaction With Relationship Power and Dating Violence Perpetration by Men and Women. Journal of Interpersonal Violence, 19 (5), 576-588.

Keddy, B., Sims, S., \& Stern, N. (1996). Grounded Theory as Feminist Research Methodology. Journal of Advanced Nursing, 23, 448-453.

Keene, S. (2019). Pleasure, Pain and Pornography: A Gendered Analysis of the Influence of Contemporary Pornography on the Lives of New Zealand Emerging Adults. Victoria University of Wellington.

Kelly, J., \& Johnson, M. (2008). Differentiation Among Types of Intimate Partner Violence: Research update and implications for interventions. Family Court Review, 46 (3), 476-499.

Kimball, E., Edleson, J., Tolman, R., Neugut, T., \& Carlson, J. (2013). Global Efforts to Engage Men in Preventing Violence Against Women: An international study. Violence Against Women, 19 (7), 924-939.

Kimmel, M. (2002). "Gender Symmetry" in Domestic Violence: A substantive and methodological research review. Violence Against Women, 8, 1332-1363.

Kimmel, M. (2012). Manhood in America: A cultural history. New York: Oxford University Press. 
Konstam, V. (2019). The Romantic Lives of Emerging Adults: Getting from I to we. New York: Oxford University Press.

Lambert, N., Negash, S., Stillman, T., Olmstead, S., \& Fincham, F. (2012). A Love That Doesn't Last: Pornography Consumption and Weakened Commitment to One's Romantic Partner. Journal of Social and Clinical Psychology, 31 (4), 410-438.

Lamont, E. (2014). Reconciling Egalitarian Ideals with Traditional Norms. Gender and Society, 28 (2).

Laroche, D. (2005). Aspects of the Context and Consequences of Domestic Violence: Situational Couple Violence and Intimate Terrorism in Canada in 1999. Institut de la Statistique du Quebec. Ontario: Canada: Government of Quebec.

Lehrner, A., \& Allen, N. (2018). A Qualitative Investigation of the Role of Gender in Young Women's Dating Violence in the United States. Violence Against Women, 24 (14), 1697-1717.

Leisring, P. (2013). Physical and Emotional Abuse in Romantic Relationships: Motivation for perpetration among college women. Journal of Interpersonal Violence, 28 (7), 1437-1454.

LeMarie, K., Oswald, D., \& Russell, B. (2016). Labelling Sexual Victimization Experiences: The role of sexism, rape myth acceptance, and tolerance for sexual harassment. Violence and Victims, 31 (2), 332-346.

Leone, J., Johnson, M., Cohan, C., \& Lloyd, S. (2004). Consequences of Male Partner Violence for Low-Income Minority Women. Journal of Marriage and Family, 66 (2), 472-409.

Lewis, S., \& Fremouw, W. (2001). Dating Violence: A critical review of the literature. Clinical Psychology Review, 21 (1), 105-127.

Lewis, S., Travea, L., \& Fremouw, W. (2002). Characteristics of Female Perpetrators and Victims of Dating Violence. Violence and Victims, 17 (5), 593.

Lindop, E., \& Goldstein, M. (2009). America in the 1960s. Twenty First Century Books.

Littleton, H., \& Axsom, D. (2003). Rape and Seduction Scripts of University Students: Implications for rape attributions and unacknowledged rape. Sex Roles, 49 (9/10), 465-475.

Littleton, H., Tabernik, H., Canales, E., \& Backstrom, T. (2009). Risky Situation of Harmless Fun? A qualitative examination of college women's bad hook-up and rape scripts. Sex Roles, 60, 793-804.

Machado, C., Caridade, S., \& Martins, C. (2010). Self-Reported Prevalence and Attitudes in a Portuguese Sample. Journal of Family Violence, 25 (1), 43-52.

Magdol, L., Moffitt, T., Caspi, A., Newmand, D., Fagan, J., \& Silva, P. (1997). Gender Differences in Partner Violence in a Birth Cohort of 21-year-olds: 
Bridging the gap between clinical and epidemiological approaches. Journal of Consulting and Clinical Psychology, 65, 68-78.

Makepeace, J. (1981). Courtship Violence Among College Students. Family Relations, 30, 97-102.

Makepeace, J. (1986). Gender Differences in Courtship Violence Victimisation. Family Relations, 383-388.

Manago, A., Ward, L., Lemm, K., Reed, L., \& Seabrook, R. (2015). Facebook Involvement, Objectified Body Consciousness, Body Shame, and Sexual Assertiveness in College Women and Men. Sex Roles, 72 (1), 1-14.

Marganski, A., \& Melander, L. (2018). Intimate Partner Violence Victimization in the Cyber and Real World: Examining the extent of cyber aggression experiences and its association with in-person dating violence. Journal of Interpersonal Violence, 33 (7), 1071-1095.

Mauthner, N., \& Doucet, A. (2003). Reflexive Accounts and Accounts of Reflexivity in Qualitative Data Analysis. Sociology, 37 (3), 413-431.

McCaw, J., \& Senn, C. (1998). Perception of cues in conflictual dating situations. Violence Against Women, 4 (5), 609-624.

McDonald, G. (2009). Retrieved 04 02, 2019 from Stuff: http://www.stuff.co.nz/dominion-post/culture/blogs/greer-2-0/2370791/TheKiwi-dating-scene

McHugh, M., \& Frieze, I. (2006). Intimate Partner Violence: New directions. Annals New York Academy of Sciences, 1087, 121-141.

McHugh, M., Livingston, N., \& Ford, A. (2005). A Postmodern Approach to Women's Use of Violence: Developing multiple and complex conceptualizations. Psychology of Women Quarterly, 29, 323-336.

McIntosh, M., \& Morse, J. (2013). Constructing Diversity in Semi-Structured Interviews. Global Qualitative Nursing Research, 2.

McKee, A. (2007). A Love That Doesn't Last: Pornography Consumption and Weakened Commitment to One's Romantic Partner. Australian Journal of Communication, 34, 87-104.

Meier, J. (2015). Johnson's Differentiation Theory: Is it really empirically supported? Journal of Child Custody, 12 (1), 4-24.

Melton, H., \& Belknap, J. (2003). He Hits, She Hits: Assessing gender differences and similarities in officially reported intimate partner violence. Criminal Justice and Behavior, 30 (3), 328-348.

Messerschmidt, J. (2018). Hegemonic Masculinity: Formulation, Reformulation, and Amplification. Rowman \& Littlefield.

Messner, M. (1990). When Bodies are Weapons: Masculinity and violence in sport. International Review of the Sociology of Sport, 25 (3), 203-220. 
Migliaccio, T. (2001). Marginalizing the Battered Male. The Journal of Men's Studies, 9 (2), 205-226.

Ministry of Justice. (2015). 2014 New Zealand Crime and Safety Survey |Te Rangahau O Aotearoa Mo Te Taihara Me Te Haumarutanga 2014: Main Findings. Wellington.

Ministry of Justice. (2018). New Zealand Crime and Victims Survey: Help create safer communities. Topline Report. Retrieved January 22, 2019 from https://www.justice.govt.nz/assets/Documents/Publications/NZCVS-2018Topline-report.pdf

Mitchell, L. (2016). Child Poverty and Family Structure: What is the evidence telling us? Auckland: Family First NZ.

Moffitt, T., \& Caspi, A. (1999). Findings About Partner Violence from the Dunedin Multidisciplinary Health and Development Study. National Institute of Justice Research. Washington: United States Department of Justice.

Moffitt, T., Krueger, R., Caspi, A., \& Fagan, J. (2000). Partner Abuse and General Crime: How are they the same? How are they Different? Criminology, 38 (1), 199-232.

Moffitt, T., Robins, R., \& Caspi, A. (2001). A Couples Analysis of Partner Abuse With Implications for Abuse-Prevention Policy. Criminology and Public Profile, 1 (1), 5.

Morgan, E. (2011). Associations Between Young Adults' Use of Sexually Explicit Materials and Their Sexual Preferences, Behaviors, and Satisfaction. Journal of Sex Research, 48 (6), 520-530.

Muehlenhard, C., \& Peterson, Z. (2005). Wanting and Not Wanting Sex: The missing discourse of ambivalence. Feminism and Psychology, 15 (1), 15-20.

Nabros, E., \& Jasinski, J. (2009). Intimate Partner Violence Perpetration Among College Students. The role of gender role and gendered violence attitudes. Feminist Criminology, 4 (1), 57-82.

Noel, N., Ogle, R., Maisto, S., \& Jackson, L. (2016). What Do Women Want? A qualitative Study of dating. Violence Against Women, 22 (2), 168-188.

Norman, D., \& Lincoln, Y. (2000). The Handbook of Qualitative Research (2nd edition). Thousand Oaks, California: Sage Publications.

Nowinski, S., \& Bowen, E. (2012). Partner Violence Against Heterosexual and Gay Men: Prevalence and correlates. Aggression and Violent Behavior, 17, 36-52.

Nybergh, L., Enander, V., \& Krantz, G. (2016). Theoretical Considerations on Men's Experiences of Intimate Partner Violence: An interview-based study. Journal of Family Violence, 31, 191-202.

NZ Herald. (2011, 06 10). The Day Sophie Died: A mothers untold story. New Zealand. 
O'Brien, W. (2011). Courageous Mother Shares Her Family's Heart-break to Help Families. Retrieved 2020 from Sophie Elliott Foundation: http://www.sophieelliottfoundation.co.nz/wpcontent/uploads/2017/11/Sophies_Legacy_PR1.pdf

Office of Film and Literature Classification. (2017). Young New Zealanders Viewing Sexual Violence: Stage 2 research report.

O'Keefe, J., Bhatti, S., Bajwa, A., DiNicolantonio, J., \& Lavie, C. (2014). Alcohol and Cardiovascular Health: The dose makes the poison ... or the remedy. Mayo Clinic Proceedings, 89 (3), 382-93.

O'Leary, K., \& Maiuro, R. (2001). Psychological Abuse in Violent Domestic Relations. New York: Springer.

O'Neal, E., Tellis, K., \& Spohn, C. (2014). When the Bedroom is the Crime Scene: To what extent does Johnson's typology account for intimate partner sexual assault. Journal of Child Custody, 11 (4), 278-303.

Ortiz, E., Shorey, R., \& Corneliua, T. (2015). An Examination of Emotion Regulation and Alcohol Use as Risk Factors for Female-Perpetrated Dating Violence. Violence and Victims, 30 (3), 417-431.

O'Sullivan, L., \& Allgeier, E. (1998). Feigning Sexual Desire: Consenting to unwanted sexual activity in heterosexual dating relationships. Journal of Sex Research, 35 (3), 234-43.

Our Watch. (2017). Putting the Prevention of Violence Against Women Into Practice: How to change the story. Melbourne: Our Watch.

Papp, L., Liss, M., Erchull, M., Godfrey, H., \& Waaland-Kreutzer, L. (2017). The Dark Side of Heterosexual Romance: Endorsement of romantic beliefs relates to intimate partner violence. Sex Roles, 76 (1), 99-109.

Paynter, A., \& Leaper, C. (2016). Heterosexual Dating Double Standards in Undergraduate Women and Men. Sex Roles, 75 (7), 393-406.

Pedersen, P., \& Thomas, C. (1992). Prevalence and Correlates of Dating Violence in a Canadian University Sample. Canadian Journal of Behavioural Science, 24 (4), 490-501.

Pence, E., \& Dasgupta, S. (2006). Re-Examining 'Battering': Are all acts of violence against intimate partners the same? Praxis International.

Pence, E., \& Paymar, M. (1993). Education Groups for Men Who Batter: The Duluth Model. New York: Springer.

Perkins, W., \& Warner, J. (2017). Sexual Violence Response and Prevention: Studies of campus policies and practices. Journal of School Violence, 16 (3), 237-242.

Peterson, K., Sharps, P., Banyard, V., Kaukinen, C., Gross, D., Decker, M., et al. (2018). An Evaluation of Two Dating Violence Prevention Programs on a College Campus. Journal of Interpersonal Violence, 33 (23), 3630-3655. 
Petter, J. (2015). Seeking Understanding of the Young Adult and their Experience of Psychodynamic Psychotherapy. A dissertation submitted to Auckland University of Technology in partial fulfilment of the requirements for the degree of Master of Psychotherapy. Auckland University of Technology.

Powell, A., Scott, A., \& Henry, N. (2020). Digital Harassment and Abuse: Experiences of sexuality and gender minority adults. European Journal of Criminology, 17 (2), 199-223.

Power, C., Koch, T., \& Jackson, D. (2006). Lovestruck: Women, romantic love and intimate partner violence. Contemporary Nurse: A Journal for the Nursing Profession, 21 (2), 174-185.

Pryor, J., \& Josie, R. (2005). What is Commitment? How married and cohabiting parents talk about their relationships. Family Matters, 71, 24.

Rapoza, K., \& Baker, A. (2008). Attachment Styles, Alcohol, and Childhood Experiences of Abuse: An analysis of physical violence in dating couples. Violence and Victims, 23 (1), 52-65.

Risman, B., \& Schwartz, P. (2002). After the Sexual Revolution: Gender politics in teen dating. Contexts, 1 (1), 16-24.

Rivera-Rivera, L., Allen-Leigh, B., Rodriguez-Ortega, G., Chavez-Ayala, R., \& Lazcano-Ponce, E. (2007). Prevalence and correlates of adolescent dating violence: Baseline study of a cohort of 7960 male and female Mexican public school students. Preventive Medicine, 44 (6), 2007.

Robertson, K., \& Murachver, T. (2007a). Correlates of Partner Violence for Incarcerated Women and Men. Journal of Interpersonal Violence, 22 (5), 639655.

Robertson, K., \& Murachver, T. (2007b). It Takes Two to Tangle: Gender symmetry in intimate partner violence. Basic and Applied Social Psychology, 29 (2), 109-118.

Rosen, K., Stith, S., Few, A., Daly, K., \& Tritt, D. (2005). A Qualitative Investigation of Johnson's Typology. Violence and Victims, 20 (3), 319-334.

Saunders, D. (1986). When Battered Women Use Violence: Husband-abuse or selfdefense? Violence and Victims, 1, 47-60.

Schulenberg, J. E., \& Schoon, I. (2012). The Transition to Adulthood Across Time and Space: Overview of special section. Longitudinal and Life Course Studies, 3 (2), 164-172.

Schwartz, S. J. (2016). Turning Point for a Turning Point: Advancing emerging adulthood theory and research. Emerging Adulthood, 4 (5), 307-317.

Scott, K., \& Straus, M. (2007). Denial, Minimization, Partner Blaming, and Intimate Partner Aggression in Dating Partners. Journal of Interpersonal Violence, 22 (7), 851-871. 
Seidman, G., Roberts, A., \& Zeigler-Hill, V. (2019). Narcissism and romantic relationship presentation on social media: The role of motivations and partner attractiveness. Personality and Individual Differences, 149, 21-30.

Shorey, R., Brasfield, H., Zapor, H., Febres, J., \& Stuart, G. (2015). The Relation Between Alcohol Use and Psychological, Physical, and Sexual Dating Violence Perpetration Among Male College Students. Violence Against Women, 21 (2), 151-164.

Shorey, R., Cornelius, T., \& Bell, K. (2008). A Critical Review of Theoretical Frameworks for Dating Violence: Comparing the dating and marital fields. Aggression and Violent Behavior, 13, 185-194.

Shorey, R., Meltzer, C., \& Cornelius, T. (2010). Motivations for Self-Defensive Aggression in Dating Relationships. Violence and Victims, 25 (5), 662-676.

Stark, E. (2007). Coercive Control: How men entrap women in personal life. New York: Oxford University Press.

Stark, E. (2006). Commentary on Johnson's "Conflict and Control: Gender symmetry and asymmetry in domestic violence. Violence Against Women, 11, 10191025.

Stark, E., \& Hester, M. (2019). Coercive Control: Update and review. Violence Against Women, 25 (1), 81-104.

Statistics New Zealand. (2017). Statistics New Zealand | Tatauranga Aotearoa. Retrieved 2018 from http://archive.stats.govt.nz/browse_for_stats/ people_and_communities/marriages-civil-unions-and-divorces/Marriages CivilUnionsandDivorces_HOTPYeDec16/Commentary.aspx

Stets, J., \& Straus, M. (1990). Gender Differences in Reporting Marital Violence nad its Medical and Psychological Consequences. In M. Straus, \& R. Gelles, Physical Violence in American Families: Risk factors and Adaptions to Violence in 8,145 Families (pp. 151-165). New Brunswick, NJ: Transaction Publishers.

Stone, J., Berrington, A., \& Falkingham, J. (2014). Gender, Turning Points, and Boomerangs: Returning home in young adulthood in Great Britain. Demography, 51 (1), 257-276.

Straus, M. (2008). Dominance and Symmetry in Partner Violence by Male and Female University Students in 32 Nations. Children and Youth Services Review, 30, 252-275.

Straus, M. (1979). Measuring Intrafamily Conflict and Violence The Conflict Tactics Scales (CTS). Journal of Marriage and the Family, 41, 75-86.

Straus, M. (2004). Prevalence of Violence Against Dating Partners by Male and Female Students Worldwide. Violence Against Women, 10 (7), 790-811. 
Straus, M., \& Gozjolko, K. (2014). "Intimate Terrorism" and Gender Differences in Injury of Dating Partners by Male and Female University Students. Journal of Family Violence, 29 (1), 51-65.

Straus, M., \& Gozjolko, K. (2016). Concordance Between Partners in "Intimate Terrosim": A comparison of two typologies. Aggression and Violent Behavior, $29,55-60$.

Straus, M., Hamby, S., Boney-McCoy, S., \& Sugarman, D. (1996). The Revised Conflict Tactics Scales (CTS2): Development and preliminary psychometric data. Journal of Family Issues, 17, 283-316.

Swan, S., \& Snow, D. (2002). A Typology of Women's Use of Violence in Intimate Relationships. Violence Against Women, 8 (3), 286-319.

Swan, S., \& Snow, D. (2006). The Development of a Theory of Women's Use of Violence in Intimate Relationships. Violence Against Women, 12 (11), 10261045 .

Swan, S., Gambone, L., Caldwell, J., Sullivan, T., \& Snow, D. (2008). A Review of Women's Use of Violence With Male Intimate Partners. Violence and Victims, 23 (3), 301-314.

Tashakkori, A., \& Teddlie, C. (2003). Handbook of Mixed Methods in Social Science and Behavioral Research. California: Sage Publications.

Te Aka Matua o te Ture Law Commission. (2019). New Strangulation Offence. Retrieved 2020 from https://www.lawcom.govt.nz/news/new-strangulationoffence

Temple, J., Weston, R., \& Marshall, L. (2005). Physical and Mental Health Outcomes of Women in Nonviolent, Unilaterally Violent and Mutually Violent Relationships. Violence and Victims, 20 (3), 335-359.

Testa, M., Livingston, J., \& VanZile-Tamsen, C. (2011). Advancing the Study of Violence Against Women Using Mixed Methods: Integrating qualitative methods into a quantitative research program. Violence Against Women, 17 (2), 236-250.

Tjaden, P., \& Thoennes, N. (1999). Violence and Threats of Violence Against Women and Men in the United States, 1994-1996. Denver: Centre for Policy Research. Ann Arbor, MI: Inter-university Consortium for Political and Social Research.

Tolman, R., Casey, E., Carlson, J., Allen, C., \& Leek, C. (2019). Global Efforts to Engage Men and Boys in Gender-Based Violence Prevention. Global Social Welfare, 6, 215-218.

Towns, A. (2007). Gender and Domestic Violence. Judges Symposium. Auckland.

Towns, A. (2009). The Culture of Cool and Being a Man: Getting in early to prevent domestic violence. Wellington. 
Towns, A., \& Adams, P. (2000). "If I Really Loved Him Enough, He Would Be Ok": Women's accounts of male partner violence. Violence Against Women, 6 (6), 558-585.

Towns, A., \& Scott, H. (2013). 'I Couldn't Even Dress the Way I Wanted.' Young Women Talk of 'Ownership' by Boyfriends: An opportunity for the prevention of domestic violence? Feminism and Psychology, 23 (4), 536-555.

Towns, A., \& Scott, H. (2008). The Culture of Cool: Getting in early to prevent domestic violence. New Zealand Family Violence Clearinghouse, Wellington.

United Nations Human Rights Council. (2011). Report of the Special Rapporteur on violence against Women, its causes and consequences, Ms. Rashida Manjoo Addendum - Mission to the United States of America.

Vic Health. (2007). Preventing Violence Before It Occurs: A framework and background paper to guide the primary prevention of violence against women in Victoria. Victorian Health Promotion Foundation.

Wang, X. (2009). Intimate Partner Violence: A survey based on 1015 college students. Tianjin, China: Tianjin Renmin Publishing House.

Wangmann, J. (2011). Different Types of Intimate Partner Violence: An exploration of the literature. University of New South Wales. Sydney: Australian Domestic and Family Violence Clearinghouse.

Weierstall, R., \& Giebel, G. (2017). The Sadomasochism Checklist: A Tool for the Assessment of Sadomasochistic Behavior. Archives of Sexual Behavior, 46 (3), 735-745.

Weldon, L. (2002). Protest, Policy, and the Problem of Violence Against Women. University of Pittsburgh Press.

White, J., \& Koss, M. (1991). Courtship Violence: Incidence in a National Sample of Higher Education Students. Violence and Victims, 6 (4), 247-256.

World Health Organisation. (2012). Intimate Partner Violence: Understanding and addressing violence against women. World Health Organisation.

Zweig, J., Yahner, J., Dank, M., \& Lachman, P. (2014). Can Johnson's Typology of Adult Partner Violence Apply to Teen Dating Violence? Journal of Marriage and Family, 76, 808-825. 


\section{APPENDICES}

\section{APPENDIX 1: KEY INFORMANTS AND THEIR ROLES AND EXPERTISE}

\begin{tabular}{|c|c|c|}
\hline Who & Role $^{9} /$ expertise & Location \\
\hline Dr Janet Fanslow & $\begin{array}{l}\text { Senior Lecturer - School of Population Health. The } \\
\text { University of Auckland. } \\
\text { - Research that can inform the direction of this } \\
\text { study. } \\
\text { - Working with victims; working with perpetrators } \\
\text { and understanding perpetrators discourse, their } \\
\text { excuses, minimisation and justifications for } \\
\text { violence. }\end{array}$ & Auckland \\
\hline Dr Alison Towns & $\begin{array}{l}\text { Director, Mt Albert Psychological Services Ltd } \\
\text { - } \\
\text { - } \quad \text { Wosearch with young adult populations. } \\
\text { subject of personal experiences of relationships } \\
\text { and violence - working with victims and } \\
\text { perpetrators. }\end{array}$ & Auckland \\
\hline $\begin{array}{l}\text { Brian Gardener } \\
\text { and Parekotuku } \\
\text { Morgan }\end{array}$ & $\begin{array}{l}\text { National Manager, National Network of Stopping } \\
\text { Violence } \\
\text { - Working with young adult populations. } \\
\text { - Working with/relating to people to establish } \\
\text { the subject of personal experiences of } \\
\text { relationships and violence - working with } \\
\text { victims and perpetrators. } \\
\text { - What patterns they are seeing in young } \\
\text { adults engaging in their services. }\end{array}$ & Wellington \\
\hline JoAnn Vivien & $\begin{array}{l}\text { National Practice Manager, Relationship Services } \\
\text { - What patterns she is seeing in young adults } \\
\text { engaging in their services. }\end{array}$ & Wellington \\
\hline Sandra Dickson & $\begin{array}{l}\text { Sexual Violence Prevention Co-ordinator, } \\
\text { Wellington Sexual Abuse Network } \\
\text { - What patterns she is seeing in young adults } \\
\text { engaging in their services. } \\
\text { - The occurrence and impacts of sexual violence in } \\
\text { relationships and conducting research in this area. }\end{array}$ & Wellington \\
\hline $\begin{array}{l}\text { Kathryn } \\
\text { McPhillips }\end{array}$ & $\begin{array}{l}\text { Clinical Manager, HELP: Support for Sexual } \\
\text { Abuse Survivors } \\
\text { - Research on sexual violence and young adults. } \\
\text { - The occurrence and impacts of sexual violence in } \\
\text { relationships and conducting research in this area. }\end{array}$ & Auckland \\
\hline Denise Lievore & $\begin{array}{l}\text { Principal Policy Analyst, Ministry of Women's } \\
\text { Affairs }\end{array}$ & Wellington \\
\hline
\end{tabular}

${ }^{9}$ This was the key informant's role at the time of the interview. 


\begin{tabular}{|c|c|c|}
\hline & $\begin{array}{l}\text { - Research with women, sexual violence, family } \\
\text { violence. }\end{array}$ & \\
\hline $\begin{array}{l}\text { Dr Rhonda } \\
\text { Pritchard }\end{array}$ & $\begin{array}{l}\text { Counsellor and member of the New Zealand Parole } \\
\text { Board } \\
\text { - Research on couple conflict, violence. } \\
\text { - Working with couples and victims/perpetrators of } \\
\text { violence as a counsellor. }\end{array}$ & Wellington \\
\hline Dr Fiona Hutton & $\begin{array}{l}\text { Senior Lecturer, School of Social and Cultural } \\
\text { Studies, Victoria University of Wellington } \\
\text { - Research in mental health, outreach work with } \\
\text { injecting drug users, the homeless and street- } \\
\text { based sex workers } \\
\text { - Experience in research with vulnerable } \\
\text { populations, on sensitive issues and in survey } \\
\text { methodology. }\end{array}$ & Wellington \\
\hline $\begin{array}{l}\text { Dr Michael } \\
\text { Roguski }\end{array}$ & $\begin{array}{l}\text { Director, Kaitiaki Research } \\
\text { - Research on family and gender-based violence, in } \\
\text { the disability sector, in crime and justice. } \\
\text { - Research with vulnerable populations, on } \\
\text { sensitive issues. }\end{array}$ & Wellington \\
\hline
\end{tabular}


MEMORANDUM

\begin{tabular}{|c|c|}
\hline TO & Fleur McLaren \\
\hline \multicolumn{2}{|l|}{ COPY TO } \\
\hline FROM & Dr. Rhonda Shaw \\
\hline DATE & 8 November 2010 \\
\hline PAGES & 1 \\
\hline SUBJECT & $\begin{array}{l}\text { Ethics Approval: No. } 17969 \\
\text { Title: 'A Fairytale ending? Violence in Intimate Dating } \\
\text { Relationships in New Zealand'. }\end{array}$ \\
\hline
\end{tabular}

Thank you for your request for ethical approval, which has been considered by the Social and Cultural Studies Human Ethics Sub-Committee.

Your application has been approved and this approval continues until the end of December 2013. If your data collection is not completed by this date you should apply to the Human Ethics Committee for an extension to this approval.

Best wishes with the research.

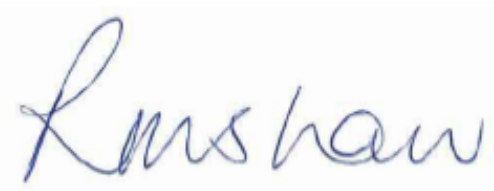

Dr Rhonda Shaw

Convener, SACS Human Ethics Committee 


\section{APPENDIX 3: SURVEY INFORMATION SHEET}

\section{Dating Experience Study}

What's this research about?

I am a Ph.D. student in Criminology at Victoria University of Wellington. As part of this degree I am undertaking a research project leading to a thesis. The project I am undertaking is examining the experiences of young adults in dating relationships.

\section{Background to the study}

Dating violence is a crucial area of study as it is a common experience for many young adults. A large number of studies on dating violence have been carried out in the United States and United Kingdom with samples of tertiary students. These international studies highlight the alarming rates of violence reported by young adults and the consequences from experiencing violence in dating relationships.

While it is well established that there are high rates of dating violence, the debate continues about what theory best describes the cause of violence between intimate partners. In recent times Johnson and colleagues have begun to develop and test a typology that tries to reconcile data that shows that violence is motivated by coercive control (feminist views) versus data that shows violence results from poor conflict resolution (conflict theorists' view) (Johnson, Janel, Leone, \& Yili, 2008).

Few New Zealand studies have investigated young adults' experience of violence and none have looked at the applicability of Johnson's statistically-derived typology and the underlying discourse across these groups.

\section{Research aim}

The aims of this proposed study are:

- to replicate Johnsons' Typology of Violence with a New Zealand sample of 18-25 year-olds, and then

- to test this typology to see if there are distinctions between these statistically derived types or whether the understanding and sense making of young adults' experiences are embedded within the same discourse of power and control. 


\section{Dating and violence, a definition for this study}

In the current study the term dating is defined as an intimate relationship lasting one or more months between never-married young adults (18-25 years). The term dating violence is defined as any young adult currently dating or dating in last the 12 months who experienced physical, psychological and/or sexual violence in their relationship.

\section{Outline of the methodology}

In this study there are two phases of data collection, an online survey and qualitative in-depth interviews. The two phases of data collection are outlined below.

The initial phase of the study is quantitative, in the form of an online survey. The online survey will be administered to university students, polytechnic students and young adults from an agency setting.

The quantitative study is not intended to be a prevalence study as the sample will not be representative of all young adults in New Zealand. The quantitative study is designed to replicate Johnson's Typologies, the frame from which I will recruit young adults to participate in the qualitative study.

The second phase of the study will be qualitative in-depth interviews with young adults recruited through the quantitative survey.

The purpose of the qualitative study is to understand the narrative around young adults' experiences of dating relationships and violence and to identify whether this discourse differs by type of violence or whether the discourse is based on the same concepts, particularly around power and control, across types.

A selection of those from the university, polytechnic and agency settings, who agree to be contacted further, will be chosen and included in the study (depending on the number willing to participate and the study sample frame).

\section{Supervision and support}

Throughout this study, I will be under the supervision of Associate Professor Jan Jordan (Programme Director, Institute of Criminology, School of Social and Cultural Studies, Humanities and Social Sciences Faculty, Victoria University of Wellington) 
and Nokuthaba Sibanda (Senior Lecturer, School of Mathematics and Statistics, Science, Faculty of Science, Victoria University of Wellington).

Dr Nokuthaba Sibanda will be the main adviser in the first phase of the study which focuses on the development, testing and analysis of the quantitative survey.

Associate Professor Jan Jordan will be the main adviser in the second phase of this study, in the development of the qualitative study and analysis. Associate Professor Jordan will also provide advice and support in current criminological thinking including research on gender and sexual violence.

This research has been assessed and approved by Human Ethics Committee - Social and Cultural Studies Subcommittee, Ethics Number 17969 


\section{APPENDIX 4: ONLINE SURVEY QUESTIONNAIRE}

\section{Dating Experience Survey}

$\mathrm{Hi}$, and welcome to the survey.

I'd like to thank you in advance for your honesty and frankness, and for taking the time to carefully consider the questions you answer. Your responses will greatly inform the research project.

Let's get started!

1. Please tick your gender

Male

Female

2. Please tick your age

Choice between 18 and 29 years

none of the above [end survey]

3. Have you ever been married?

Yes [end survey]

No

4. Consent: Completion and submission of this survey is deemed as consent for this information to be included in the research project. Identifying details will be changed to ensure your anonymity. Please click "I consent" if you are happy for your anonymous information to be included.

I consent [enter survey]

I do not consent [exit survey - to the thank you page]

5. Please tick a box below that describes your 'relationship status'

Currently in a relationship [send to questions on current relationship 6] 
Not currently in a relationship but have been in a relationship in the last 12 months [send to questions on past relationship 7]

Have not been in a relationship in the last 12 months [end survey]

6. Thinking about your current relationship, how long have you been together?

Less than one month [end survey]

About 1 month

About 2 months

Three to five months

Six months to eleven months

About a year

More than a year, but less than 2 years

About 2 years

More than 2 years, but less than 4 years

Four years or more

[to question 9]

7. Thinking about the past 12 months, have you had a relationship that lasted more than 1 month?

Yes [to question 5c]

No [end survey]

8. Thinking about that relationship, how long did it last?

About 1 month

About 2 months

Three to five months 
Six months to eleven months

About a year

For the rest of the survey, when questions refer to your partner, think about the relationship you were just describing that occurred in the last 12 months and lasted for one month or more. [go to question 10]

9. Thinking of this current relationship, what was the gender of your current partner

Male

Female

10. Thinking of this past relationship, what was the gender of your ex-partner

Male

Female

11. How old is your current partner / ex-partner?

12. What is the 'status' of this relationship?

Dating

Engaged

Married [end survey]

13. Thinking about your current or ex partner, would you say he/she... $1=$ Yes, $0=$ No

$\ldots$ is jealous or possessive,

... tries to provoke arguments,

... tries to limit your contact with family and friends,

... insists on knowing who you are with at all times,

... calls you names or puts you down in front of others,

... makes you feel inadequate,

... shouts or swears at you, 
$\ldots$ frightens you, and

... prevents you from knowing about or having access to the family income even when you ask.

14. Thinking about how you feel towards your current or ex partner, would you say you ... 1=Yes, $0=$ No

$\ldots$ are jealous or possessive,

... try to provoke arguments,

... try to limit their contact with family and friends,

... insist on knowing who they are with at all times,

... call them names or put them down in front of others,

... make them feel inadequate,

... shout or swear at them,

... frighten them

... prevent them from knowing about or having access to the family income

15. No matter how well a couple gets along, there are times when they disagree, get annoyed with the other person, want different things from each other, or just have spats or fights because they are in a bad mood, are tired, or for some other reason. Couples also have many different ways of trying to settle their differences. This is a list of things that might happen when you have differences.

Please circle how many times you did each of these things in the past year, and how many times your partner did them in the past year. If you or your partner did not do one of these things in the past year, but it happened before that, circle "7."

I insulted or swore at my partner $\mid$ My partner did this to me

I threw something at my partner that could hurt

I twisted my partner's arm or hair 
I had a sprain, bruise, or small cut because of a fight with my partner

Made my partner have sex without a condom

I pushed or shoved my partner

I used force (i e hitting, holding down, or using a weapon) to make my partner have oral or anal sex

I used a knife or gun on my partner

I passed out from being hit on the head by my partner in a fight

I called my partner fat or ugly

I punched or hit my partner with something that could hurt

I destroyed something belonging to my partner

I went to a doctor because of a fight with my partner

I choked my partner

I shouted or yelled at my partner

I slammed my partner against a wall

I needed to see a doctor because of a fight with my partner, but I didn't

I beat up my partner

I grabbed my partner

I used force (like hitting, holding down, or using a weapon) to make my partner have $\operatorname{sex}$

I stomped out of the room or house or yard during a disagreement

I insisted on sex when my partner did not want to (but did not use physical force)

I slapped my partner

I had a broken bone from a fight with my partner 
I used threats to make my partner have oral or anal sex

I burned or scalded my partner on purpose

I insisted my partner have oral or anal sex (but did not use physical force)

I accused my partner of being a lousy lover

I said something to spite my partner

I threatened to hit or throw something at my partner

I felt physical pain that still hurt the next day because of a fight with my partner

I kicked my partner

I used threats to make my partner have sex

16. Please circle how many times you did each of these things in the past year, and how many times your partner did them in the past year. If you or your partner did not do one of these things in the past year, but it happened before that, circle "7."

My partner followed or spied on me?

I followed or spied on my partner

My partner sent me unsolicited letters or written correspondence?

I sent my partner unsolicited letters or written correspondence?

My partner made unsolicited phone calls to me?

I made unsolicited phone calls to my partner?

My partner stood outside my home, school, or workplace?

I stood outside my partner's home, school, or workplace?

My partner showed up at places I was even though he or she had no business being there?

I showed up at places my partner was even though I had no business being there?

My partner left unwanted items for me to find? 
I left unwanted items for my partner to find?

My partner tried to communicate in other ways against my will?

I tried to communicate in other ways against my partner will?

My partner vandalised my property or destroyed something I loved?

I vandalised my partner's property or destroyed something my partner loved?

If respondent answered "yes" to one or more of the above questions, then she/he is asked:

2. Has anyone ever done any of these things to you on more than one occasion?

How frightened were you by these things [perpetrator] did to you?

Very frightened

Somewhat frightened

Just a little frightened

Not really frightened

17. Which city do you currently live in?

18. What ethnic group do you affiliate with?

NZ European

Māori

Other ethnicity

Don't know

Refused

19. What is your highest education qualification?

No qualification

Any school qualification 
Trade/Advanced trade certificate

NZ certificate or diploma

Local polytechnic certificate or diploma

Technicians certificate

Nursing certificate or diploma

Teachers certificate or diploma

Bachelor degree

Postgraduate and honours qualification (including masters and doctorates)

Other qualification

Don't know

Refused

20. Where are you attending, studying or enrolled at school

Victoria University of Wellington

Whitereia

WelTec

Other (please state)

21. Employment status

Mark as many spaces as you need to show all the ways you yourself got income in the last 12 months

Wages, salary, commissions, bonuses, etc, paid by my employer

Self-employment, or business I own and work in

Interest, dividends, rent, other investments

Regular payments from ACC or a private work accident insurer 
Government benefits, government income support payments, war pensions, or paid parental leave

Other sources of income, counting support payments from people who do not live in my household

No source of income during this time

Income

22. From all the sources of income you marked in question 21 , what will the total income be:

Loss

Zero income

$\$ 1-\$ 10,000$

$\$ 10,001-\$ 40,000$

$\$ 40,001$ or more

23. What ethnicity is your partner?

NZ European

Māori

Other ethnicity

Don't know

Refused

24. Thinking about your current relationship, or the last relationship you had (that you answered the rest of the questions about), what is your living status?

Live with partner and no one else

Live with partner in flat with others

Live with partner and parents 
Live alone

Other

And finally...

25. How did you find out about this survey?

In class

Poster on campus

Through an agency

Word of mouth - through a friend

Social networking site

Other 


\section{APPENDIX 5: IN-DEPTH INTERVIEW INFORMATION SHEET}

Seeking your consent to be involved in research

This sheet talks about a type of research that I would like to do with young adults about their experiences in intimate relationships. The University makes sure that researchers do the right thing by making them explain who they are, say what they intend to do and then discuss with people what might happen if they agree to be in the research project.

No research can begin until the people who have been asked to be involved are clear about what is going to happen and have agreed to this in writing.

\section{What's this research about?}

I am a Ph.D. student in Criminology at Victoria University of Wellington. As part of this degree I am undertaking a research project leading to a thesis. The project I am undertaking is examining the experiences of young adults in intimate relationships.

What does participating in this study involve?

I am inviting people who:

$\square$ are between the ages of 18 to 25 years;

$\square$ are currently in an intimate relationship that has lasted for more than one month or have been in an intimate relationship in the past 12 months that lasted for more than one month; and

$\square$ have never been married.

If you replied yes to all three questions then you have the chance to participate in this study. Participating in this study means meeting with me one-on-one to talk about your experience of intimate relationships and your attitudes about relationships. The interview will last between 60-90 minutes. You are welcome to bring a support person to the interview if you would feel more comfortable with someone else there. 


\section{What happens if you want to stop part way through?}

You can pull out of the interview at anytime. If you choose to withdraw then all information gathered on you, or that you contributed will be destroyed.

What happens to what you tell me?

Responses collected will form the basis of my research project and will be put into a written report on an confidential basis. It will not be possible for you to be identified personally. Only grouped responses will be presented in this report.

All responses collected from you will be kept confidential. I would like to record the interview on a digital recorder. No other person besides me will have access to your digitally recorded interview. Your responses will be locked away where only I can access them.

The thesis will be submitted for marking to the School of Social and Cultural Studies and deposited in the University Library. It is intended that one or more articles will be submitted for publication in scholarly journals. Interview data will be destroyed five years after the end of the project.

If you have any questions or would like to receive further information about the project, please contact me or my supervisor, Associate Professor Jan Jordan.

Do you have any further questions at this point?

[Contact details provided]

This research has been assessed and approved by Human Ethics Committee - Social and Cultural Studies Subcommittee

Ethics Number 17969 


\section{APPENDIX 6: INTERVIEW GUIDE}

\section{Experience of dating}

- Can you tell me a little bit about your current relationship?

- How would you describe your current relationship? Satisfied, not sure it will last...

- Thinking of your own experience of dating, how do you know if things are going well?

- How do you know if things aren't going well

- What is he/she like with their mates?

- Did you notice negative things after you broke up or during the relationship - at what point did you notice this negative stuff?

\section{Influences on dating}

- Do you think there are social or cultural influences those influences how you think relationships should be, such as friends, family, the media? What are they saying relationships should be like?

- Is this your experience in your relationship? Do you think these expectations make you behave in a particular way?

- Do you think some of these things/influencers encourage people to treat their partners badly?

- Is there pressure to be in a relationship? To not being single? Is this the same for guys/girls?

- Is there pressure to have sex? Is this the same for guys/girls?

\section{Ever experienced violence}

- Have you ever been in a relationship where you felt unsafe or something negative happened $^{10}$ ?

- Can you tell me what happened?

\footnotetext{
${ }^{10}$ I did not use the word violence unless they used the term, otherwise the conversation was in the context of the situation they were sharing.
} 
- When did the violence happen?

- Can you tell me about the first time it happened?

- What motivated the violence?

- How did the violence impact on you? Were you scared by this behaviour?

- How did the violence change during the course of the relationship?

- Were there any barriers/reasons that stopped you leaving / getting help?

What is violence - how you define it / trying to get a sense of what people think about violence in dating relationships

- What would you define as dating violence? Who commits dating violence? Why do people commit dating violence?

Thoughts on jealousy and control

- There are some things that motivate violence like jealousy, control or conflict.

- Do these things resonate with you in your current relationship? Or in past relationships?

- Can you describe how they played out in that relationship? 


\section{APPENDIX 7: COPY OF THE CONSENT FORM}

Victoria University wants you and me to understand what you are agreeing to, if you say YES to taking part in this research project. Please go through each statement and tick the box if you agree with each statement.

I have read and understood the information provided above about this research project.

$\square$ I have had an opportunity to ask questions and to have them answered.

$\square$ I understand that notes will be taken during the interviews and that they may also be audio-taped.

$\square$ I understand that I may withdraw myself or any information that I have provided for this project at any time prior to 31 August 2011.

$\square$ If I withdraw, I understand that all relevant information including tapes and transcripts, or parts thereof, will be destroyed.

$\square$ I agree to take part in this research.

$\square$ I wish to receive a summary of the results of the research (please tick one):

If you have ticked all the boxes above and are happy to participate in this study, please sign and date the box below.

I am eighteen years or over. I would like to be a part of this research project. I have no questions or concerns about the information we have just gone through.

\section{Signed}

Date

Name of participant (please print clearly)

Thank you for your time. If you have signed, welcome to the project!

Fleur McLaren

his research has been assessed and approved by Human Ethics Committee - Social and Cultural Studies Subcommittee

Ethics Number 17969 


\section{APPENDIX 8: DESCRIPTION OF THE INTERVIEW PARTICIPANTS AND THEIR PARTNERS TYPOLOGY CLASSIFICATION}

\begin{tabular}{|c|c|c|c|c|c|c|c|}
\hline $\begin{array}{l}\text { Participant } \\
\text { name }\end{array}$ & $\begin{array}{l}\text { Participant } \\
\text { typology }\end{array}$ & $\begin{array}{l}\text { Partner's } \\
\text { typology }\end{array}$ & $\begin{array}{l}\text { Combined } \\
\text { typology }\end{array}$ & $\begin{array}{l}\text { Current } \\
\text { or ex } \\
\text { partner }\end{array}$ & Violence & Injury & $\begin{array}{l}\text { Sexual } \\
\text { coercion }\end{array}$ \\
\hline Rebecca & SCV & SCV & SCV & Ex & $\begin{array}{l}\text { Minor } \\
\text { only }\end{array}$ & None & $\begin{array}{l}\text { Willing } \\
\text { unwanted }\end{array}$ \\
\hline Sarah & SCV & $\mathrm{SCV}$ & SCV & Current & $\begin{array}{l}\text { Minor } \\
\text { only }\end{array}$ & Minor & $\begin{array}{l}\text { Coerced } \\
\text { sexual } \\
\text { activity }\end{array}$ \\
\hline Sue & NV & SCV & SCV & Current & $\begin{array}{l}\text { Minor } \\
\text { only }\end{array}$ & No & $\begin{array}{l}\text { No } \\
\text { mention }\end{array}$ \\
\hline Kathryn & $\mathrm{NV}$ & $\mathrm{SCV}$ & $\mathrm{SCV}$ & Ex & $\begin{array}{l}\text { Minor } \\
\text { only }\end{array}$ & None & $\begin{array}{l}\text { Willing } \\
\text { unwanted }\end{array}$ \\
\hline Mary & SCV & $\mathrm{NV}$ & SCV & Ex & $\begin{array}{l}\text { Minor and } \\
\text { Severe }\end{array}$ & Minor & $\begin{array}{l}\text { Coerced } \\
\text { sex }\end{array}$ \\
\hline Emma & NV & SCV & SCV & Current & $\begin{array}{l}\text { Minor } \\
\text { only }\end{array}$ & Minor & $\begin{array}{l}\text { Coerced } \\
\text { sexual } \\
\text { activity }\end{array}$ \\
\hline Beth & SCV & SCV & SCV & Current & $\begin{array}{l}\text { Minor } \\
\text { only }\end{array}$ & No & $\begin{array}{l}\text { Willing } \\
\text { unwanted }\end{array}$ \\
\hline $\begin{array}{l}\text { Drake } \\
\text { (male) }\end{array}$ & NV & SCV & $\mathrm{SCV}$ & Ex & $\begin{array}{l}\text { Minor } \\
\text { only }\end{array}$ & No & $\begin{array}{l}\text { No } \\
\text { mention }\end{array}$ \\
\hline Jack (male) & SCV & SCV & SCV & Ex & $\begin{array}{l}\text { Minor } \\
\text { only }\end{array}$ & $\begin{array}{l}\text { Minor } \\
\text { injury }\end{array}$ & $\begin{array}{l}\text { Coerced } \\
\text { sexual } \\
\text { activity }\end{array}$ \\
\hline $\begin{array}{l}\text { Grant } \\
\text { (male) }\end{array}$ & NV & $\begin{array}{l}\text { SCV } \\
\text { same sex }\end{array}$ & SCV & Ex & $\begin{array}{l}\text { Minor } \\
\text { only }\end{array}$ & $\begin{array}{l}\text { Minor } \\
\text { only }\end{array}$ & $\begin{array}{l}\text { Tapped } \\
\text { sex }\end{array}$ \\
\hline Jessica & NV & IT & IT & Ex & $\begin{array}{l}\text { Minor and } \\
\text { Severe }\end{array}$ & $\begin{array}{l}\text { Minor } \\
\text { and } \\
\text { Severe }\end{array}$ & $\begin{array}{l}\text { Coerced } \\
\text { sexual } \\
\text { activity }\end{array}$ \\
\hline Sammy & NV & IT & IT & Ex & $\begin{array}{l}\text { Minor and } \\
\text { Severe }\end{array}$ & Minor & $\begin{array}{l}\text { Tapped } \\
\text { sex }\end{array}$ \\
\hline Karen & $\mathrm{NV}$ & IT & IT & Ex & $\begin{array}{l}\text { Minor and } \\
\text { Severe }\end{array}$ & Minor & $\begin{array}{l}\text { Forced } \\
\text { sexual } \\
\text { activity }\end{array}$ \\
\hline Laura & $\mathrm{NV}$ & IT & IT & Ex & $\begin{array}{l}\text { Minor and } \\
\text { Severe }\end{array}$ & Minor & $\begin{array}{l}\text { No } \\
\text { mention }\end{array}$ \\
\hline $\begin{array}{l}\text { Ricky } \\
\text { (male) }\end{array}$ & IT & SCV & VR & Ex & $\begin{array}{l}\text { Minor and } \\
\text { Severe }\end{array}$ & $\begin{array}{l}\text { Minor } \\
\text { and } \\
\text { Severe }\end{array}$ & $\begin{array}{l}\text { No } \\
\text { mention }\end{array}$ \\
\hline
\end{tabular}




\begin{tabular}{|c|c|c|c|c|c|c|c|}
\hline $\begin{array}{l}\text { Regan } \\
\text { (male) }\end{array}$ & IT & NV & IT & Ex & $\begin{array}{l}\text { Minor and } \\
\text { Severe }\end{array}$ & Minor & $\begin{array}{l}\text { Coerced } \\
\text { sexual } \\
\text { activity }\end{array}$ \\
\hline
\end{tabular}




\section{DATING EXPERINCE STUDY}

... a fairytale ending?

Welcome to the Project

$\mathrm{Hi}$, my name is Fleur McLaren and I am a Ph.D. student at Victoria University of Wellington.

As part of this degree I am undertaking a research project to understand the different experiences young kiwi's are having in dating relationships.

There's a lot in the media about 'hooking up' - movies, video clips, reality t.v. all shape what we expect from our relationships - what the perfect partner look likes, what they do, how they talk, even down to the amazing sex you'll have together!

\section{So, what does all this mean for real life hook ups?}

That is what I am trying to find out. Is the old-fashioned dating culture dead or are experiences still similar when we get down to the nitty-gritty?

\section{I want to hear from you}

What's your experience of dating, what influences your expectations, and how do you make sense of your positive and negative dating experiences.

Are you living the fairytale... or is your relationship shaping up like a horror show!

\section{If you're interested in sharing your experience in \\ a anonymous online survey then click the survey tab and take part.}

I'd love to have you be part of this project.

\section{Contact details}

Fleur McLaren

PhD Student

School of Social and Cultural Studies

Victoria University of Wellington

New Zealand

datingexperiencestudy@hotmail.com
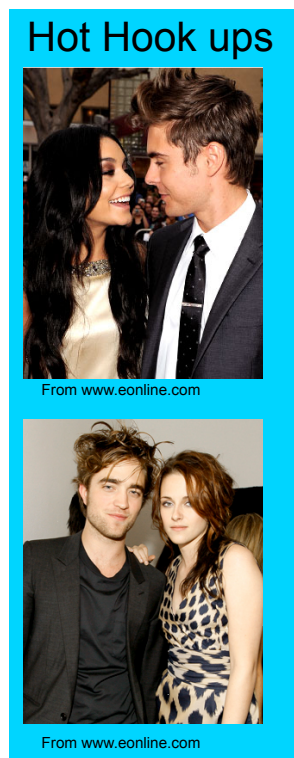

From www.eonline.com

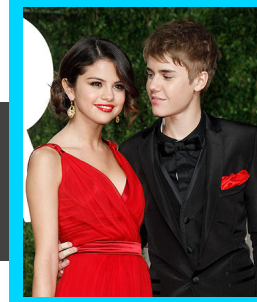

From www.stuff.co.nz

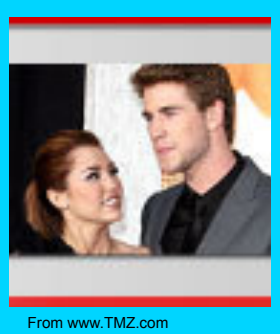

Site map Admin 